\title{
An experimental approach to linguistic representation
}

\author{
Holly P. Branigan \\ Department of Psychology, University of Edinburgh, Edinburgh, EH8 9JZ, \\ United Kingdom \\ holly.branigan@ed.ac.uk \\ http://www.ed.ac.uk/profile/holly-branigan \\ Martin J. Pickering \\ Department of Psychology, University of Edinburgh, Edinburgh, EH8 9JZ, \\ United Kingdom \\ martin.pickering@ed.ac.uk \\ http://www.ed.ac.uk/profile/martin-pickering
}

\begin{abstract}
Within the cognitive sciences, most researchers assume that it is the job of linguists to investigate how language is represented, and that they do so largely by building theories based on explicit judgments about patterns of acceptability-whereas it is the task of psychologists to determine how language is processed, and that in doing so, they do not typically question the linguists' representational assumptions. We challenge this division of labor by arguing that structural priming provides an implicit method of investigating linguistic representations that should end the current reliance on acceptability judgments. Moreover, structural priming has now reached sufficient methodological maturity to provide substantial evidence about such representations. We argue that evidence from speakers' tendency to repeat their own and others' structural choices supports a linguistic architecture involving a single shallow level of syntax connected to a semantic level containing information about quantification, thematic relations, and information structure, as well as to a phonological level. Many of the linguistic distinctions often used to support complex (or multilevel) syntactic structure are instead captured by semantics; however, the syntactic level includes some specification of "missing" elements that are not realized at the phonological level. We also show that structural priming provides evidence about the consistency of representations across languages and about language development. In sum, we propose that structural priming provides a new basis for understanding the nature of language.
\end{abstract}

Keywords: language production; linguistics; mental representation; psycholinguistics; semantics; structural priming; syntax

The cognitive science of language is concerned with both linguistic representations and how those representations are used in processing. All researchers, whether nominally psychologists or linguists, should seek to address both questions. In practice, however, linguists have focused largely on representation and used a single method (acceptability judgments) to investigate it, whereas psychologists have not investigated representation but instead imported linguistic theories into their accounts. In this paper, we argue instead that researchers need not, and should not, be restricted to acceptability judgments when investigating linguistic representation.

This proposal is not new but was previously just a theoretical possibility. It now appears, however, that structural priming-the tendency to repeat linguistic structure across utterances - allows researchers to investigate linguistic representations in a way that has many advantages over acceptability judgments. Most important, it has now reached maturity, in that hundreds of studies have used this method; and many of them are informative, not merely about language processing, but also about linguistic representations themselves. In fact, we argue that evidence from structural priming supports quite specific proposals about linguistic structure relating to syntax and semantics, so that it can be used to develop linguistic theory and discriminate among competing accounts. Thus, the dominance of acceptability judgments can be ended, and the understanding of linguistic representation can develop to a greater extent than before.

This paper describes our theoretical claims, linguistic account, and applications. In section 1, we motivate the use of structural priming to investigate mental representation and present the advantages of structural priming over acceptability judgments. In section 2 , we consider what the extensive evidence using this method tells us about linguistic representation. Section 3 discusses the implications of our account.

\section{Why a psychological account of linguistic structure is now possible}

A complete theory of human language requires characterization of both people's linguistic representations and the processes that operate over those representations. Therefore, issues of representation and processing are in 
principle of interest to both linguists and psychologists, albeit from different perspectives. In practice, however, linguistic representation (in particular, with respect to syntactic and semantic structure) has for the last four decades been largely the domain of linguists and has been studied primarily using a single approach in which linguists or their informants make explicit metalinguistic judgments about the grammatical (or semantic) acceptability of individual sentences-henceforth, acceptability judgments. Such judgments constitute the dataset upon which theories of linguistic representation are based.

In this paper, we propose that the representations underlying language use need not and, in fact, should not be investigated only via such judgments. Rather, we suggest that they can be examined directly through a behavioral measure that has been used widely in psychological research to investigate the representation of a range of information types. This method is priming: If processing one stimulus affects the subsequent processing of another stimulus, then these stimuli share some aspect of their representation. Hence, structural priming effects, where processing one utterance affects the processing of another utterance that shares an aspect of linguistic structure but is otherwise unrelated, provide evidence about linguistic representation. In the classic demonstration, Bock (1986) had participants repeat active or passive sentences and then describe pictures depicting transitive events. She found that they were more likely to use a passive target sentence (e.g., The church is being struck by lightning) after repeating a passive prime sentence (The referee was punched by one of the fans) than after repeating an active

Holly Branigan is a Professor of Psychology of Language and Cognition at the University of Edinburgh. She has published more than 65 journal articles and in numerous other publications on topics relating to language processing. Her research investigates the cognitive processes and representations that underlie language production and interactive language use, with a particular focus on syntactic structure, and aspects of the relationship between psycholinguistics and linguistics. Her work addresses these issues in a wide range of populations, including monolingual and bilingual adults, and typically and atypically developing children. In 2014-15, she was recipient of a British Academy/Leverhulme Trust Senior Research Fellowship.

Martin Pickering is a Professor of the Psychology of Language and Communication at the University of Edinburgh. His research focuses on the representation and processing of language and, in particular, on the mechanisms underlying dialogue as a form of joint action. He has published more than 140 journal articles on such topics as language comprehension during reading, self-monitoring during speech, language switching in bilinguals, and turn-taking in language and music. He served as editor of the Journal of Memory and Language, was recipient of the Experimental Psychology Society mid-career award, and is a Fellow of the Royal Society of Edinburgh.
(One of the fans punched the referee). As subsequent studies have demonstrated, these effects appear to arise from repetition of aspects of abstract linguistic structure and occur largely outside of awareness (Pickering \& Ferreira 2008).

They cannot be explained in terms of repetition of particular words. Bock (1989) showed that participants tended to use prepositional object $(\mathrm{PO})$ dative sentences (The girl is handing a paintbrush to the man) rather than double object (DO) sentences (The girl is handing the man a paintbrush) after a dative sentence that did not include to (The secretary baked the cake for her boss). Therefore, priming could not be due to word repetition, because the $\mathrm{PO}$ and DO target sentences share all words except to.

We also can rule out explanations couched entirely in terms of meaning. First, the alternative responses (e.g., $\mathrm{PO}$ and DO, or active and passive) denote the same events, in that they both can be used to describe the same picture. Second, Messenger et al. (2012b) found priming between sentences describing different event types (e.g., Experiencer-Theme: The king is being ignored by the bear, and Agent-Patient: The doctor gets licked by the cow). Additionally, Hartsuiker and Westenberg (2000) found that Dutch participants repeated the order of auxiliary and main verb (was geblokkeerd ["was blocked"] vs. geblokkeerd was ["blocked was"]), even though they do not differ in meaning. Moreover, the effects cannot be explained by repetition of metrical structure, because The girl is handing a paintbrush to the man was not primed by Susan brought a book to study, though it was primed by the metrically equivalent Susan brought a book to Stella (Bock \& Loebell 1990). Overall, these results are consistent with priming of representations that are specified for syntactic information but not semantic, lexical, or phonological information. This conclusion is supported by studies showing priming of many other syntactic constructions, such as noun-phrase structure (Cleland \& Pickering 2003) and verb-particle placement (Konopka \& Bock 2009).

Priming, however, is also informative about other aspects of linguistic structure, including many components of semantics including thematic roles, quantification, and information structure. It occurs in diverse languages (e.g., English, Mandarin, Basque) and between languages, and in children, non-native speakers, amnesiacs, and aphasics. It has been found using many experimental methods, as well as in natural conversation (Pickering \& Ferreira 2008).

It also occurs in comprehension, as indicated by choice of structure (e.g., Branigan et al. 2005), reading time (e.g., Traxler et al. 2014), predictive eye movements (Arai et al. 2007; Thothathiri \& Snedeker 2008a), event-related potentials (ERPs) (Ledoux et al. 2007), and brain activity revealed by functional magnetic resonance imaging or fMRI (Segaert et al. 2012). Priming of comprehension usually involves participants selecting between analyses that have different meanings (e.g., high- or low-attached prepositional phrases [PPs]; Branigan et al. 2005), though experiments investigating predictions in "visual-world" paradigms and those using fMRI are exceptions. When both meaning and syntax differ across conditions, it becomes much harder to relate any priming effects to linguistic representation.

Importantly, structural priming occurs from comprehension to production (Branigan et al. 2000; Potter \& 
Lombardi 1998) to a similar extent as within production (Bock et al. 2007; Tooley \& Bock 2014), and from production to comprehension to a similar extent as within comprehension (Branigan et al. 2005). Moreover, studies of priming effects within comprehension, within production, and between production and comprehension implicate common neural architectures (Menenti et al. 2011; Segaert et al. 2012; Segaert et al. 2013). These findings are particularly important for justifying the relevance of priming to representation. We therefore believe we can use structural priming effects to develop a psychologically motivated theory of syntactic representation and the way in which it relates to semantic representation. But before sketching this account in section 2 , we need to justify why such an account is possible in principle.

\subsection{The reality of linguistic representation}

The nature of linguistic representation is of fundamental interest for experimental psychologists who are concerned with language, because people must represent linguistic structure to use language. Psychological theories of language, therefore, must specify the representations that speakers and hearers use, as well as the processes that operate over those representations, in the same way that theories of visual cognition specify the representations that perceivers construct as they interpret scenes (Biederman 1987).

Understanding the nature of linguistic representation has also been the central goal of most theoretical linguistics, at least since the publication of Syntactic Structures (Chomsky 1957). Linguists have attempted to provide grammars for natural languages (i.e., precise descriptions of the relationships that may hold between linguistic expressions). Some linguists view such grammars as characterizations of essentially "platonic" objects that have nothing to do with the human mind (e.g., Katz 1981; see also Langendoen \& Postal 1984). Any such platonic linguistics is not our concern. For most linguists, however, grammars are envisaged as the knowledge that underlies speakers" and hearers' use of language: "Linguistics is that branch of psychology that focuses its attention on one specific cognitive domain and one faculty of mind, the language faculty" (Chomsky 1980, p. 4). Its primary aim is to construct a grammar that is psychologically real, in the sense that "the grammar corresponds to the speaker's internal representation of that domain" (Bresnan \& Kaplan 1982 , p. xxiii). Our concern is the nature of this internal representation.

Among linguists, however, there are different views on the relationship between this representation and language processing (see Lewis \& Phillips 2015). One possibility is that the grammar is drawn upon directly during processing. This is clearly the simplest approach, requiring the fewest additional assumptions. Under this approach, evidence about the representations involved in language processing is clearly relevant to linguistic theory; "linguistic" and "psycholinguistic" representations would be the same. (Any discrepancies between evidence from processing and acceptability judgments would be due to factors such as processing limitations that are explicable in terms of generally accepted cognitive assumptions [Lewis \& Phillips 2015].)
Other linguists assume that the grammar is not used directly in processing - in other words, that the grammar and the language processing system form two distinct systems. For these researchers, processing is assumed to involve linguistic representations, but the nature of those representations need not constrain their theories. The kinds of theory involving two such systems might include those that specify a form of "universal grammar" that is available early in development and inputs into the grammars of specific languages but does not continue to be represented later in development (e.g., Clahsen \& Muysken 1986), or theories in which underlying representations are compiled into different representations that are used during processing online (e.g., Berwick \& Weinberg 1984; Fodor 1983). Moreover, linguistically motivated theories tend to seek to describe the language using as parsimonious a representational system as possible (e.g., Chomsky 1995), an approach that will not necessarily be compatible with the representations used in language processing (e.g., Croft 2001; Jackendoff 2002).

In all such cases, however, the representations used by the processor remain an object of enquiry that critically pertains to the speaker's internal representation of the linguistic domain (and any theory that assumes two systems of representation must explain how the two systems are related). Our goal is to consider alternative (experimental) methods to acceptability judgments that potentially address the linguistic representations implicated in language processing. Evidence from such methods cannot disprove the existence of other representations. But a theory that does not assume inaccessible representations, however, is more parsimonious than one that does. If the two representational systems are assumed only because of apparent incompatibility between acceptability judgment and processing data, then it is preferable to assume a single representation, and that different methods tap into the same representation in slightly different ways (see Lewis \& Phillips 2015).

We therefore assume - in the absence of compelling evidence to the contrary - that there is a single representational system for language structure, which is implicated during language processing, and that people do not have other (inaccessible) mental representations of language structure. If any such representations were to exist, they would clearly be of interest. But they do not form part of our account, and it is for theories that propose such representations to motivate them and to specify the mapping between them and those used in processing.

To characterize the knowledge that speakers and hearers draw on, researchers from both experimental psychological and theoretical linguistic backgrounds might, in principle, use evidence from many different sources, including judgments of grammaticality and meaning, and evidence from language acquisition and perceptual experiments (Chomsky 1965, p. 37). However, Chomsky is unconvinced by the use of processing evidence to investigate linguistic representation. In an important footnote, he says:

One common fallacy is to assume that if some experimental result provides counter-evidence to a theory of processing that includes a grammatical theory $\mathrm{T}$ and parsing procedure $\mathrm{P} \ldots$... then it is $\mathrm{T}$ that is challenged and must be changed. The conclusion is particularly unreasonable in the light of the fact that in general there is independent (so-called linguistic) 
Branigan and Pickering: An experimental approach to linguistic representation

evidence in support of $\mathrm{T}$ while there is no reason at all to believe that $\mathrm{P}$ is true. (Chomsky 1981, p. 283, footnote 39)

This provides a justification for ignoring experimental data. In practice, most linguists have adopted this approach. Therefore, they have tended to base their theories (particularly of syntax) primarily on evidence from acceptability judgments; in particular, they have tended to ignore psychological data suggesting that people process sentences using representations that differ from those proposed by linguists (see Fodor et al. 1974; Wasow \& Arnold 2005).

\subsection{Why acceptability judgments are not enough}

Acceptability judgments involve native speakers of a language deciding whether sentences are acceptable or unacceptable. Traditionally, linguists who investigate whether sentences are grammatical usually refer to them as grammaticality judgments: Sentences judged grammatical should be licensed by the grammar; sentences that are judged ungrammatical should not be licensed by the grammar. Acceptability judgments are a convenient source of data, because all that is required is a native speaker. They can provide evidence about the set of possible sentences that comprise a language, and are assumed to give evidence concerning the structure of speakers' internalized knowledge of language (e.g., Chomsky 1986). Acceptability judgments historically have provided a fertile source of evidence for hypotheses about the nature of linguistic representation, but they pose many concerns.

Some are surmountable and relate to how they typically have been used (e.g., Gibson \& Fedorenko 2013). For example, linguists standardly ask a single informant about the acceptability of a few sentences. It is possible, however, to conduct acceptability judgments as a wellcontrolled psycholinguistic experiment using many (varied) sentences, using naïve participants, controlling for plausibility, and randomizing presentation order. It is also possible to control for effects of previous exposure or judgments (sentences appearing more or less acceptable when the construction is repeated; e.g., Levelt 1972; Luka \& Barsalou 2005; Snyder 2000), a phenomenon presumably related to structural priming.

Next, acceptability judgments face the problems associated with any explicit task. The informant's judgments may reflect decision-making biases. This concern is exacerbated when the informant is the researcher or has knowledge of the theoretical questions under investigation. Moreover, the informant may not interpret terms such as grammatical or acceptable as the linguist intends. To all of these concerns, linguists may respond that traditional methods are adequate, because they have not led to many errors (e.g., Sprouse et al. 2013) and because native-speaker linguists can immediately detect erroneous judgments used in theory building (e.g., Phillips 2009), but controversy remains.

However, acceptability judgments face more fundamental problems. Most obviously, they can be used to study linguistic representations only in certain populations, because they can be elicited only from speakers who are capable of making metalinguistic judgments. For example, they cannot be used with children younger than 3 (nor indeed with many 3-year-olds; Ambridge \& Rowland 2013; McDaniel \& Smith Cairns 1998). Hence, acceptability judgments cannot be used to address some fundamental representational questions.

Another far-reaching problem is source ambiguity (Hofmeister et al. 2013). There is no reason to believe that acceptability judgments offer privileged access to linguistic representation in a way that other methods do not. Acceptability judgments are the results of linguistic and cognitive processes, by which people attempt to process sentences and then make metalinguistic judgments on the results of those acts of processing (e.g., someone cannot understand a sentence or finds it jarring and, therefore, assumes it is unacceptable). Thus, they implicate the same linguistic representations involved in all acts of processing. Therefore, it is not possible to tell whether any judgment of unacceptability reflects ungrammaticality, low probability, or unprocessability. For example, Bresnan (2007) found that acceptability judgments for sentences were affected by those sentences' probability of occurrence. Equally, people often judge center-embedded sentences (e.g., The rat that the cat that the dog bit chased fled) as unacceptable, yet most theorists follow Chomsky (1965) in assuming they are grammatical and that people's judgments reflect processing difficulty. Similarly, garden-path sentences (e.g., The horse raced past the barn fell) often are judged unacceptable, yet most theorists assume this is because people initially misanalyze them and fail to recover (Bever 1970). In these cases, linguists might argue that there are clear explanations for why they are judged unacceptable (complexity, confusability, ambiguity).

In other cases, however, the explanation for why a sentence is unacceptable is more contentious - for example, whether the unacceptability of What did who visit? reflects a syntactic violation (Chomsky 1995) or processing difficulty (Hofmeister et al. 2013). Conversely, linguists sometimes argue that acceptable sentences are not grammatical (e.g., It was I; Sobin 1997). In this respect, acceptability judgments are susceptible to the same challenges as processing data: The data are compatible with particular grammar-processor pairings, not just with particular grammars. An explanation of which sentences are acceptable and which are not therefore seems to require a theory of processing alongside a theory of grammaticality.

A more fundamental problem is that, even if it could somehow be determined that a particular set of acceptability judgments indexed grammaticality, such judgments directly determine only set membership. That is, they determine weak generative capacity: which sentences are members of the set of sentences licensed by a grammar, and which sentences are not. ${ }^{1}$ However, they cannot by themselves determine linguistic structure. To draw inferences about linguistic structure, they need to be combined with tests about constituency.

As widely acknowledged, however, constituency structure tests are inconsistent and problematic in many ways. Textbooks introducing such tests standardly warn that they produce contradictory results. To give some examples: Coordination tests support the existence of constituents (e.g., an NP-NP constituent in The woman gave the child a cake and the $\operatorname{dog}$ a bone) that other tests such as topicalization and it-cleft do not (and in this case, most linguistic theories ignore the coordination test). Ellipsis and questionshort answer tests may support constituents (e.g., baked a cake in I said he baked a cake and in fact he did so/What did he do? Baked a cake) when topicalization and it-cleft 
tests do not $\left({ }^{*}\right.$ I said he baked a cake and baked a cake he ${ }^{*}$ It is baked a cake that he, where ${ }^{*}$ indicates ungrammaticality). Ellipsis tests yield obviously problematic results (e.g., China is a country Tom wants to visit, and he will if he gets the money suggests that China ... visit is a constituent; Kempson et al. 1999). These are not unusual or isolated examples, and even the most basic assumptions about constituency (e.g., the structure of simple transitive sentences) show different results for different tests. Moreover, the basic rationale for why these specific tests should tap constituent structure remains unclear (Berg 2009). In fact, it has been proposed that they are more appropriately considered as structural heuristics rather than structural diagnostics (Payne 2006).

Most important, the use of acceptability judgments, with or without the application of constituency tests, has yielded no consensus at all about linguistic representation. For example, theories associated with the transformational tradition (i.e., following accounts such as Chomsky 1981; Chomsky 1995) assume syntactic representations of considerable complexity, including many more branching nodes than words, a large number of empty categories, and extensive movement of constituents. Such representations can be interpreted as involving many syntactic levels (if movement is interpreted as taking place in stages) and associations between the syntactic representations and other representations such as Logical Form or LF) and Phonetic Form or PF, which themselves input into meaning and sound. These theories also make broad assumptions such as binary branching. In contrast, theories such as Head-Driven Phrase Structure Grammar (HPSG; Pollard \& Sag 1994) make very different assumptions, with simpler, flatter trees, and few if any empty categories. Some theories assume grammatical functions play a central role (e.g., Lexical-Functional Grammar [LFG]; Kaplan \& Bresnan 1982), whereas others do not. Other theories assign a key role to "constructions" (e.g., Goldberg 1995) or allow overlapping constituents (e.g., Steedman 2000). Additionally, there is little agreement about whether there is a clear distinction between syntactic and lexical information, or whether most syntactic information is stored alongside lexical items. Acceptability judgments have not been able to adjudicate between these alternatives, except insofar as one set of rules or constraints that can generate the same set of sentences is "better" by some metric such as parsimony or learnability (and even on these grounds, there is disagreement).

In sum, acceptability judgments have been more successful in inspiring accounts of linguistic representation than in discriminating among those accounts. They have inherent and fundamental limitations: Judgments can be influenced easily by nonlinguistic factors; they cannot be used at all with some populations; and, most importantly, they do not provide direct evidence about structure. Given these concerns, researchers concerned with linguistic representations should not rely solely on such judgments; they should call on additional methodologies that are directly sensitive to structure and that avoid the limitations discussed above.

\subsection{Psycholinguistic approaches to linguistic representation}

Is there a different approach to linguistic representation that is based more directly on psycholinguistic methods?
Researchers have intermittently proposed that some form of experimental method may be informative about linguistic representation (and not merely processing). In the 1960s, psychologists attempted to relate processing difficulty to linguistic complexity (e.g., number of transformations; Chomsky 1965) using reaction time measures (McMahon 1963; Miller 1962; Miller \& McKean 1964). However, it proved very difficult to control for other potentially relevant factors. For example, a passive might take longer to process than an active because a passive involves an additional transformation (hence, greater representational complexity), or alternatively because of length, word frequency, local or global ambiguity, and so on.

Other experimental studies tested for the existence of empty categories, as assumed by some linguistic theories (e.g., Chomsky 1981) but not others. McElree and Bever (1989) found that people were faster to decide whether a critical phrase had occurred in a sentence if an empty category (or "gap") corresponding to the phrase occurred at the end of the sentence than if the sentence had no empty category. They argued that comprehenders reactivated the empty category at its location, and hence, that empty elements are mentally represented (see also Nicol \& Swinney 1989). But these results do not require empty categories, and may instead be due to semantic processes. Pickering \& Barry (1991) accordingly argued against the representation of empty categories in sentences such as In which pot did you put the cup? because people appear to relate in which pot to the verb put as soon as they reach the verb (Sag \& Fodor 1994; Traxler \& Pickering 1996). Gibson and Hickok (1993), however, proposed an account of these data in which people project (i.e., predict) an empty category when they reach the verb. In accord with Chomsky (1981), Pickering and Barry's data can be explained by either a bottom-up parser (parsing procedure $\mathrm{P}_{1}$ ) using a grammar without empty categories (grammatical theory $\mathrm{T}_{1}$ ), or a top-down parser $\left(\mathrm{P}_{2}\right)$ using a grammar with empty categories $\left(\mathrm{T}_{2}\right)$. More recent attempts to use processing evidence to adjudicate among competing linguistic theories of ellipsis, quantification, and scalar implicature have faced analogous problems (Lewis \& Phillips 2015).

Other types of experimental work are relevant, in principle, to linguistic representation but do not provide the basis for a general methodology for understanding linguistic representation. Sprouse et al. (2012) found that the acceptability of sentences violating island constraints (e.g., What do you wonder whether John bought? ) is unrelated to measures of working-memory capacity and therefore argued that such island constraints are likely to constitute part of grammar. Such research may constrain linguistic theories but relates to quite specific phenomena. Some researchers have used patterns of agreement errors (the road to the islands; e.g., Bock \& Miller 1991) to draw conclusions supporting linguistic frameworks incorporating movement and empty categories (Franck et al. 2010), but others assume that they are informative about processing mechanisms (e.g., the scope of utterance planning; Gillespie \& Pearlmutter 2013). Research that uses young children's errors to infer their underlying representations runs into the same problem of distinguishing representational from processing explanations (Ambridge \& Rowland 2013). Studies using ERPs show different signatures for implausible versus ungrammatical sentences (e.g., Kutas \& Hillyard 
1980; Osterhout \& Holcomb 1992), but it is unclear whether there is a specifically semantic or syntactic component in the ERP waveform (e.g., Kim \& Osterhout 2005; Nieuwland et al. 2013). Likewise, fMRI studies do not unambiguously identify brain regions that are associated with particular levels of linguistic representation (Price 2010).

In fact, most psychologists of language largely have shied away from making claims about linguistic representation and instead adopt the representations proposed by linguists. A classic example is Frazier's (1987) garden-path theory, which assumes that comprehenders initially select the syntactically simpler analysis of an ambiguous utterance. The theory makes specific syntactic assumptions (e.g., ternary branching structure is possible), which affect its predictions. However, experiments concerned with the theory (e.g., Frazier \& Rayner 1982) have not attempted to test whether these assumptions are correct. Many alternative accounts of parsing are, if anything, even less tempted to encroach on the territory of linguistic representation (e.g., MacDonald et al. 1994).

In sum, linguists and psychologists agree that linguistic structure is mentally represented. But acceptability judgments are an imperfect and limited way of investigating such representations, and psychological approaches have not provided a general method for investigating linguistic representation. However, we now propose that structural priming is a very promising method that can be used systematically to address many linguistic questions.

\subsection{Can structural priming be used to investigate linguistic representation?}

Priming effects occur when processing a stimulus with particular characteristics affects subsequent processing of another stimulus with the same or related characteristics (Schacter 1987). Such effects are found pervasively throughout cognition. In visual perception, for example, object recognition can be facilitated by previous exposure to a stimulus with shared visual features (Biederman \& Cooper 1991). Psychologists use such effects to investigate the nature of underlying representations. The logic underlying priming methodologies is that exposure to a prime stimulus facilitates (or inhibits) particular representations, making them more (or less) amenable to subsequent reuse if they can be applied to a subsequent target stimulus. $^{2}$ If processing of a stimulus $A$ is affected by prior processing of $B$ to a greater extent than by prior processing of $C$, then the representation underlying $A$ is more similar to the representation underlying $B$ than it is to the representation underlying $C$. By careful investigation, we can determine how $A$ and $B$ are related, and use this relationship to inform a general theory of representation. For example, Biederman and Cooper manipulated the extent to which prime and target stimuli shared visual attributes such as vertices and convex/concave components, and used their results to propose a theory of visual object representation.

Such effects provide an implicit measure of representation that is independent of any explicit response (e.g., regarding well-formedness, presence of particular characteristics, similarity). They occur without awareness or explicit recall of the prime stimulus and are generally believed to be automatic and resource free (e.g., Dehaene et al. 1998; Forster \& Davis 1984). In other words, priming effects arguably implicate a direct relationship between representation and behavior.

Priming paradigms have been applied to language extensively. For example, participants are faster at judging that a target stimulus is a word (e.g., nurse) if they have just responded to a semantically (or associatively) related prime word (doctor) than an unrelated word (table; Meyer \& Schvaneveldt 1971). By manipulating the relationship between prime and target, researchers have constructed detailed models of the psychological representation of lexical entries (McNamara 2005). For example, MarslenWilson et al. (1994) found priming between words that shared a semantically transparent stem (e.g., observationobservant) but not between words that had a common historical derivation but did not share a semantically transparent stem (e.g., apart-apartment) and used these findings to argue that the former had a decomposable (bimorphemic) representation whereas the latter did not. They noted that this psychological evidence contrasted with theoretical and historical linguistic analyses.

We argue that priming can be used similarly to investigate the representation of any aspect of linguistic structure. Thus, we could demonstrate changes in some aspect of behavior (e.g., likelihood of a particular response, response time, patterns of brain activity) following a sentence with particular characteristics and draw inferences about the representations that underlie the prime and target, without requiring participants to make any explicit judgment.

Experiments using structural priming paradigms avoid many problems typically associated with acceptability judgments. They standardly use many sentences and many naïve participants, control for plausibility differences and effects of previous exposure, and randomize presentation order (though we have noted that these controls can be applied to acceptability judgments). Because they use implicit behavioral measures, they can avoid decisionmaking biases and problems about informants' interpretation of acceptable and unacceptable (or grammatical and ungrammatical). For the same reason, they can be used to investigate representations in participants who cannot make appropriate metalinguistic judgments or who are indeed unable to make any explicit response-for example, young children or language-impaired patients. Furthermore, because priming is based on the processor recognizing that two utterances are related, such experiments provide evidence that goes beyond set membership. Finally, investigations of priming between comprehension and production are directly informative about representation (rather than aspects of processing that are specific to production or comprehension). ${ }^{3}$

A possible concern is that priming between two sentences may tap into a level of representation that is distinct from another linguistic representation that is inaccessible to priming (e.g., a "deep structure" as in Chomsky 1965). As stated above, however, our goal is to characterize the linguistic representations implicated in language use (and we have argued against inaccessible representations; Section 1.1). Of course, any such objection equally applies to the use of acceptability judgments, which also involve processing and might fail to access such representations.

Another concern is identifying which aspect of structure that priming taps into. For example, speakers might tend to repeat POs or DOs (Bock 1986) because they are primed to repeat syntactic structure, or because they are 
primed to repeat aspects of meaning, thematic role order (e.g., Theme-Recipient vs. Recipient-Theme), or order of animate/inanimate entities, among other possibilities. In some cases, it is possible to exclude alternative explanations within an experiment. In other cases, we should seek converging evidence across experiments, whereby alternative explanations are ruled out systematically (as has been done for POs/DOs; see sect. 1, para. 3-4, and sect. 2).

A different concern is that structural priming itself may be susceptible to processing influences. Obviously, it may not be sensitive to linguistic relationships under all conditions - for example, if the target occurs too long after the prime. Participants also may sometimes fail to demonstrate priming because of processing limitations (e.g., children may sometimes be unable to produce complex structures, despite having the relevant linguistic representations). For these participants, it may be important to use priming paradigms that minimize processing requirements or do not require an overt response (e.g., using ERPs and fMRI; Ledoux et al. 2007; Segaert et al. 2012).

A more serious problem would occur if an effect that mimicked structural priming arose for reasons that are not informative about linguistic representation. In the case of acceptability judgments and when using comprehension data (e.g., reading times), we have noted that conclusions about linguistic representation (i.e., T) might depend on assumptions about processing (i.e., P). But it is hard to see how the explanation of priming could depend on processing assumptions.

Priming also could occur for reasons other than similarity of linguistic representation. For example, comprehending a garden-path sentence might be easier following another, unrelated garden-path sentence, because comprehenders are primed to adopt more complex or less frequent analyses. Equally, speakers might be more likely to produce a rare (or less felicitous) structure after encountering another rare (or less felicitous) structure. But such effects should be more general than effects due to structural priming and could be distinguished with careful experimentation.

A final concern is that most demonstrations of priming in production relate to choices between sentence forms, and so rely on the existence of structural alternatives. It is hard to use priming in production to investigate the representation of sentences in which no relevant alternative exists, or in which one alternative is highly infrequent or infelicitous. This simply means that priming in production cannot be used to investigate all structures. On some occasions, priming in comprehension may present an alternative.

\subsection{Summary}

There has been a historical division between a theoretical linguistic focus on representation and a psychological focus on processing. Research on representation has relied almost exclusively on acceptability judgments, which have provided a fertile source of data for developing hypotheses but have many limitations and do not provide unambiguous diagnostics that can discriminate among alternative hypotheses. Most methods grounded in psychology (or neuroscience) have not themselves provided such diagnostics. However, we have argued that structural priming is different: It provides evidence that is directly informative about mental representation.
We propose that acceptability judgments can be used with appropriate controls alongside structural priming (and perhaps other experimental methods; see sect. 1.3) as a means of developing representational hypotheses. ${ }^{4}$ But they should not be the final arbiters for discriminating among hypotheses. Instead, researchers should where possible use structural priming to test hypotheses. In many cases, evidence from structural priming will converge with evidence from acceptability judgments, and hence provide strong support for specific representational claims. In other cases, priming evidence will adjudicate between competing linguistic accounts (whether different analyses of the same construction within the same broad linguistic framework, or analyses based on very different linguistic assumptions). Where acceptability judgment and priming evidence do not converge, evidence from priming should be favored, especially when acceptability judgments do not produce clear evidence.

We have made this argument in principle. But we suggest that there is now sufficient evidence from structural priming experiments to outline a psychologically motivated account of syntactic aspects of linguistic representation and their relationship to semantics and the lexicon. We base this account on specific structural priming findings but argue that it is also compatible with traditional linguistic evidence and that it discriminates among theories based on such evidence.

\section{An outline theory of syntax and its interfaces based on structural priming}

To explain our account, we consider the representation of $A$ book was begun by every linguist, under an interpretation in which each linguist began writing a (possibly) different book. We focus on information that appears relevant to syntactic representation, either as part of the syntactic representation itself or by interfacing with the syntactic representation.

First, people must represent semantic information (roughly corresponding to the speaker's intended "message"; Levelt 1989). Importantly, this includes propositions represented in terms of predicates and their arguments. In our example, one proposition encodes a complex event structure involving the initiation of an event of writing. This writing event is associated with two thematic roles: an Agent that undertakes the act of writing and a Theme that is written. The Agent of the writing act is also the Agent who initiates this act. There is also quantificational information that every linguist has wider scope than $a$ book, and information structure specifying that a book is emphasized.

We also assume that people represent syntactic and lexical information about the words that are used and how they are arranged. Thus, people represent that the sentence includes the words $a$ and book, in that order, as well as information about larger units of structure (e.g., that $a$ and book form a constituent). Importantly, elements expressed in the message may not always correspond straightforwardly to elements expressed in the syntax and to lexical content (e.g., there is no word expressing the writing event; cf. Jackendoff 2002). Finally, people represent the relationship between these different types of information and sound (phonology, intonation, etc.). 
Branigan and Pickering: An experimental approach to linguistic representation

Our account has the following basic properties (see Fig. 1). It distinguishes representations specifying semantics from those specifying syntax. There is a single semantic level of representation that encodes information about quantificational scope relations, information structure, and thematic structure, including missing elements (i.e., elements that do not correspond to an element that is uttered). There is a single syntactic level of representation that draws on well-formedness constraints (or rules) specifying local relations with respect to linear order as well as hierarchical relations. The syntactic level of representation includes syntactic category information but not semantic information (e.g., thematic roles) or lexical content. There is no syntactic movement, but some elements that are not uttered are represented in the syntax. The syntactic level is separate from a single sound-based level of representation that encodes phonology, syllabic structure, and metrical information (to which we refer using the blanket term "phonological information"). We assume one sound-based level, because there is insufficient evidence to discriminate different levels (see Sevald et al. 1995; Tooley et al. 2014a).

\subsection{Syntactic representation}

We begin by motivating the syntactic level of representation, because this is the level for which there is most evidence from priming. Our account assumes a single level of syntax that includes constituent structure. There are no separate levels containing, for example, reordered constituents (e.g., Deep Structure) or unordered constituents (e.g., incorporating hierarchical structure but not linear order). In addition, this level does not incorporate quantificational information (which instead forms part of the semantic representation).

First, syntactic representations do not contain semantic information. This claim is supported by evidence of priming between sentences involving different types of events, predicates, and entities. Bock and Loebell (1990) found that intransitive active sentences with a $b y$-phrase expressing a location (The foreigner was loitering by the broken traffic light) primed transitive passive sentences where the by-phrase expressed an Agent (The boy was woken by an alarm clock). Messenger et al. (2012b) showed priming between passive sentences that involved different thematic roles (e.g., The girl is being scared by the pig and The king is being ignored by the bear both primed The doctor gets licked by the cow); as we discuss below, these results cannot be explained by closed-class word repetition (see also Bock 1989). Both studies found the same magnitude of priming when primes and targets did not involve the same thematic roles as when they did.

Additionally, priming occurs when the alternatives involve no discernible semantic difference. Hartsuiker and Westenberg (2000) found structural priming for the order of the auxiliary and main verb in Dutch, even though they involve the same words and do not express different meanings. Konopka and Bock (2009) similarly showed priming for the position of the particle in meaning-identical sentences involving phrasal verbs (e.g., pulled the sweater off vs. pulled off the sweater). Ferreira (2003) found priming for the presence versus absence of the complementizer that (e.g., The mechanic mentioned the car could use a tune-up vs. The mechanic mentioned that the car could use a tune-up).

These studies also demonstrate that the relevant representations are not bound intrinsically to open-class lexical content: Priming occurs between sentences that share no such content. Nor are they bound to closed-class content (e.g., The secretary baked a cake for her boss and The secretary brought a cake to her boss primed The girl is giving a paintbrush to the man to the same extent [Bock 1989; see also Pickering \& Branigan 1998]). Other experiments show

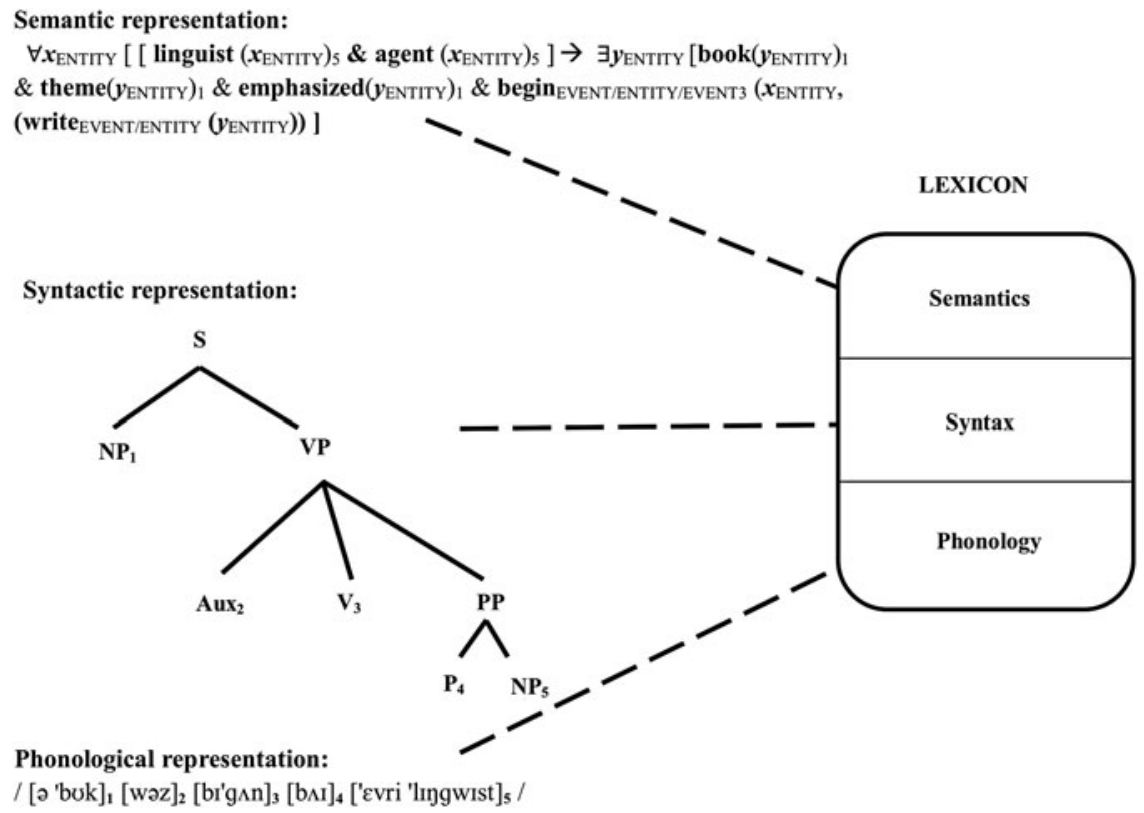

Figure 1. Outline model of the representation of A book was begun by every linguist, incorporating a single level of semantic representation, a single level of syntactic representation, and a single level of phonological representation. Dashed lines indicate bindings between components of semantic, syntactic, and phonological structural representations, and the semantic, syntactic, and phonological components, respectively, of lexical entries. Subscripts indicate coindexation between levels. 
structural priming between sentences containing a mismatch between syntactic structure and the verb's subcategorization requirements (e.g., The waitress exists the book to the monk primes PO responses [Ivanova et al. 2012a; see also Ivanova et al. 2012b]) If syntactic representations were bound to lexical content, priming should have occurred only when the syntactic properties of the words were compatible with the sentence structure.

The finding that priming occurs between sentences with different phonological content (e.g., for-to in Bock 1989; was showing-showed in Pickering \& Branigan 1998) to the same extent as priming between sentences with the same phonological content (to-to; showed-showed) also implies that syntactic representations do not contain word-level phonological information. Additionally, Bock and Loebell (1990) showed that priming did not occur based on metrical structure (e.g., Susan bought a book for Susan primed POs, but Susan brought a book to study did not).

Hence, priming evidence supports the existence of abstract syntactic representations. It also suggests that these are shallow and monostratal in a way that corresponds at least roughly to the assumptions of Culicover and Jackendoff (2005) and many other nontransformational theories (e.g., Gazdar et al. 1985; Goldberg 1995; Pollard \& Sag 1994; Steedman 2000). It does not support a second, underlying level of syntactic structure or the syntactic representation of empty categories associated with the movement of constituents in some transformational analyses. Thus, Bock and Loebell's (1990) finding of priming from intransitive (active) locatives to passives implies that these structures share syntactic representations, which we take to be noun-phrase (NP; the foreigner, the boy), verb (including auxiliary; was loitering, was woken), and PP (by the broken traffic light, by an alarm clock). Our account contrasts with many syntactocentric linguistic theories, which assume distinct syntactic representations for passives and intransitive locatives. Specifically, transformational accounts assume that the passive involves an empty category associated with the subject (the boy) immediately after the verb (woken), whereas intransitive locatives do not involve an empty category. Converging evidence supporting our account comes from Flett's (2006) finding that Spanish speakers tended to repeat the order of the subject and verb in unaccusative sentences to the same extent following unergative and unaccusative primes, which transformational accounts assume involve distinct syntactic representations (with unaccusatives but not unergatives involving subject movement and an associated empty category).

Similarly, priming from transitive locatives to POs implies that these constructions share syntactic representations (Bock \& Loebell 1990), whereas many transformational accounts assume that they have different structures such that the PP appears as a sister to the verb node in POs (because it is a complement) but as a sister to a higher V' node in locatives (because it is an adjunct). The only accounts in which POs and transitive locatives have the same representation are where the structure is shallow and simple, in the sense that there are nodes for the verb, NP, and PP, but nothing else. Likewise, Wittenberg (2014) found (bidirectional) priming between POs/ DOs and "light-verb" sentences (e.g., The kidnapper gives the government an ultimatum/an ultimatum to the government), whereas transformational accounts assume distinct representations, with POs/DOs - unlike light-verb sentences-involving a V-trace (Hale \& Keyser 1993; 2002; Wittenberg et al. 2014).

Syntactic representations also are monostratal in the sense that they represent hierarchical and linear relations simultaneously. Pickering et al. (2002) showed that sentences involving the same hierarchical relations but different linear relations did not prime each other. Participants were no more likely to produce a PO (involving V NP PP order) following a "shifted" PO (the same constituents in V PP NP order; e.g., The racing driver showed to the helpful mechanic the damaged wheel) than following an intransitive sentence. Pappert and Pechmann (2014) found similar results in German, in which the shifted order is much less unusual.

The syntactic representations capture local relationships between a "mother" and its constituent "daughter(s)" (e.g., a VP comprising a verb and two NPs), independent of the larger context in which the phrase appears (e.g., that the VP occurs within a subordinate clause), or the internal structure of the subphrases that constitute it (e.g., that the first NP comprises a determiner, adjective, and noun). ${ }^{5}$ This assumption is consistent with any approach to grammar that distinguishes within- and between-phrasal relations, such as context-free grammars with maximal projections. It is motivated by evidence that priming occurs between sentences that share local structure but differ at other levels. Branigan et al. (2006) found priming when the prime involved a DO or PO structure in a main clause (e.g., The racing driver showed the helpful mechanic the flat tyre), and the target involved a subordinate clause (e.g., The rumours alleged that the patient showed the doctor his scar) and vice versa. In fact, priming occurred to the same extent whether the prime and target involved the same or different clause types, implying that the same representations were involved whenever a DO or PO structure was used, irrespective of the larger context (see also Melinger \& Cleland 2011).

Likewise, priming occurs between sentences that differ in detailed structure (i.e., constituents' internal structure). Pickering and Branigan (1998) found PO/DO priming when the internal structure of complement NPs differed between prime and target (e.g., omission or inclusion of adjectives: The racing driver showed the torn overall to the manager primed The patient showed his spots to the doctor). Moreover, Fox Tree and Meijer (1999) found equivalent priming for POs and DOs whether the VPs in prime and target had the same internal structure (i.e., both included or did not include a subordinate relative clause) or different internal structure (i.e., one involved a subordinate relative clause and the other did not). This finding also demonstrates that priming is not based on a sequence of phrasal categories (i.e., without hierarchical structure).

Finally, traditional theories of language production refer to grammatical functions such as subject (e.g., Garrett 1975), for example assuming that they have their own "deep" level of representation (corresponding roughly to F-structure in LFG; Kaplan \& Bresnan 1982) that is independent of constituent structure. Many linguistic theories also assume some form of representation of grammatical functions, even those that attempt to develop monostratal syntax (see Culicover \& Jackendoff 2005 pp. 152, 538). For John loves Mary, speakers

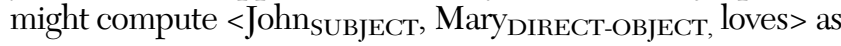


an unordered representation separate from [NP John] [vP [v loves] [NP Mary]]. Such a representation would be incompatible with our assumption of a single syntactic level.

Some priming studies have been interpreted in terms of grammatical functions (see sect. 2.4). Bock et al. (1992) argued that speakers repeat mappings of animacy features (encoded in semantic representations) to grammatical functions (e.g., inanimate to subject). But participants also might have repeated mappings of animacy features to word-order positions (e.g., inanimate to first NP). Chang et al. (2003) reported priming effects that could have reflected a tendency to repeatedly assign thematic roles (e.g., Location) to grammatical functions (e.g., direct object) or to word-order positions (e.g., immediately following the verb). Cai et al. (2012) found some evidence (in Mandarin) for separate priming from thematic roles to grammatical functions and from thematic roles to wordorder positions, and argued that grammatical functions should be incorporated into the constituent structure representation (e.g., [NP John $\left.n_{\text {SUBJECT }}\right][\mathrm{vP}$ [v loves $]$

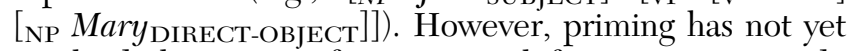
resolved the status of grammatical functions, so we do not incorporate them into Figure 1.

2.1.1. Missing elements. We have argued that priming evidence does not support the existence of empty categories associated with the movement of NPs or verbs in syntactic structure and have proposed a monostratal account involving a single level of syntax linked to a single level of semantics and a single level of phonology. But within this account, some elements that are not phonologically represented may be syntactically represented. In fact, priming may allow us to determine cases in which missing elements are represented syntactically and cases in which they are not. More generally, priming potentially allows us to address the syntactic representation of sentences in which the semantics and phonology are misaligned: Does the syntax align with the former or the latter?

We first consider ellipsis. Syntactic accounts of ellipsis assume that elided elements are represented syntactically (as well as semantically; e.g., Hankamer 1979; Merchant

(a)

give $_{2}\left(\operatorname{man}\left(x_{\mathrm{ENTITY}}\right)_{1} \&\right.$ agent $\left(x_{\mathrm{ENTITY}}\right)_{1} \&$ $\operatorname{money}\left(y_{\mathrm{ENTITY}}\right)_{3} \&$ theme $\left(y_{\mathrm{ENTITY}}\right)_{3}$ \& $\left.\operatorname{charity}\left(z_{\mathrm{ENTITY}}\right)_{4} \& \operatorname{recipient}\left(z_{\mathrm{ENTITY}}\right)_{4}\right)$

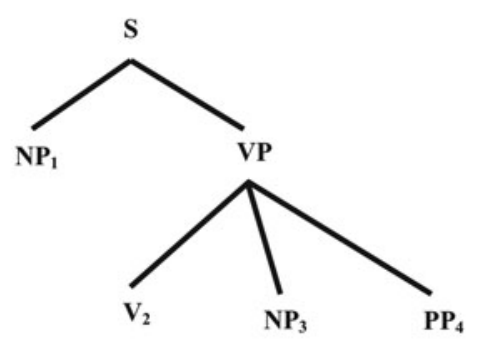

... [the man $]_{1}[\text { gave }]_{2}[\text { some money }]_{3}$
2001); semantic accounts assume that they are represented semantically but not syntactically (e.g., Fiengo \& May 1994). Consider The charity needed support so the man gave some money, in which the semantic representation specifies the Agent (the man), Theme (some money), and Recipient (the charity), whereas the phonological representation specifies the Agent and Theme but not the Recipient. According to syntactic accounts, the syntactic representation includes a PP (e.g., V NP PP), so that it is aligned with the semantic (but not the phonological) representation, as in Figure 2a; according to semantic accounts, it does not include a PP (e.g., V NP), so that it is aligned with the phonological (but not the semantic) representation, as in Figure $2 \mathrm{~b}$.

Cai et al. (2015) found that missing (elided) NP arguments in Mandarin are represented syntactically. They showed that full DO targets were primed by DOs in which the Theme was missing (e.g., Niuzai mai-le yiben shu hou song-gei-le shuishou; "The cowboy bought a book and later gave the sailor [the book]") to the same extent as by DOs in which the Theme was not missing (Niuzai mai-le yiben shu hou song-gei-le shuishou naben shu; "The cowboy bought a book and later gave the sailor the book"). Similarly, PO targets were equally primed by POs with or without the Theme. These results suggest that the missing element was represented in the syntactic structure in the same way as an overtly expressed element, as in Figure 2a, and are therefore consistent with syntactic accounts.

In contrast, Cai et al. (2013) found that elided VPs in Mandarin are not represented syntactically. They showed that full DO targets were not primed by DOs in which the VP was elided (e.g., Fuwuyuan xiang jie-gei shuishou naba qiang. Yinwei haipa reshi, chushi que bu xiang, "The waitress would like to lend the sailor the gun. Being afraid of getting into trouble, the chef would not like to [lend the sailor the gun]"), compared to when the VP was overtly expressed (Chushi que bu xiang jie-gei shuishou naba qiang, "The chef would not like to lend the sailor the gun"). These results suggest that the internal structure

(b)
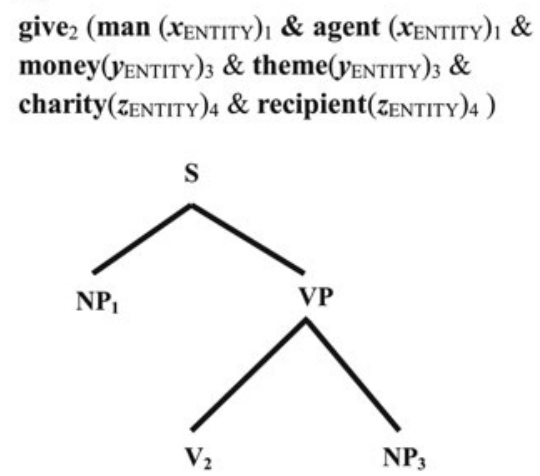

... [the man $]_{1}[\text { gave }]_{2}[\text { some money }]_{3}$

Figure 2. Accounts of the representation of the missing-argument sentence The man gave some money. The italicized sentences represent the phonological representation. In (a), the meaning and thematic role of the missing argument are specified in the semantic representation (top), and the missing argument is specified in the syntactic representation (center); in (b), the meaning and thematic role of the missing argument are specified in the semantic representation, but the missing argument is not specified in the syntactic representation. Subscripts indicate coindexation between levels. 
Branigan and Pickering: An experimental approach to linguistic representation

of the elided VP (V NP NP) was not represented syntactically and are therefore consistent with semantic accounts.

Other priming evidence similarly indicates that some semantically specified elements are not specified syntactically. Raffray et al. (2014) examined sentences such as The celebrity began drinking the champagne (full VP sentence) and The celebrity began the champagne (coerced sentence). The semantic representation for both sentences specifies the nature of the predicate (i.e., drinking) involved in the event. However, Raffray et al. found that The celebrity began the champagne did not prime production of The clerk began reading the report, suggesting that the coerced sentence has no syntactic element corresponding to the missing predicate drinking. Instead, it behaved like a noncoerced sentence such as the celebrity began the speech (in which there is no missing predicate, given that the speech refers to an event).

In addition, Pappert and Pechmann (2013) showed that PO/DO sentences (e.g., Die Sekretärin backte ihrem Chef einen Kuchen, "The secretary baked her boss a cake") primed benefactive sentences (e.g., Der Soldat hob seinem Freund eine Zigarette auf, "The soldier saved his pal a cigarette"), despite their semantic differences: $\mathrm{PO} /$ DO sentences involve a simple transfer event, whereas benefactives involve a complex event comprising a creation or preparation event and a potential transfer or change of possession event (Shibatani 1996). These results suggest that they are nevertheless syntactically represented in the same way. ${ }^{6}$

Overall, these results provide evidence for the syntactic representation of some but not all missing elements (i.e., elements that are semantically but not phonologically represented). Moreover, they imply that patterns of structural priming can determine which missing elements are syntactically represented and which are not. More generally, they suggest that priming can help determine the extent to which syntactic representations are aligned with semantic or phonological representations.

\subsection{Semantic representation}

Our model proposes that the semantic level of representation contains at least specifications of quantificational information, information structure, and thematic roles. We assume a single level of semantic representation, because most studies have focused on distinguishing different aspects of semantics from syntax and have not sought to distinguish among aspects of semantics. We first consider the representation of quantificational information and its relation to thematic roles. Raffray and Pickering (2010) reported that priming is sensitive to a level of semantic representation specifying quantifier scope (see also Chemla \& Bott 2015; Feiman \& Snedeker 2016; Viau et al. 2010). They presented participants with doubly quantified prime sentences such as Every kid climbed a tree, which are ambiguous between a universal-wide interpretation (every kid climbed a potentially different tree) and an existential-wide interpretation (every kid climbed the same tree), together with a disambiguating picture that forced one or other interpretation. When they then read a different doubly quantified target sentence that also involved a universally quantified Agent and existentially quantified Patient (Every hiker climbed a hill), participants tended to interpret it in the same way.
Participants did not tend to repeat the interpretation of Every hiker climbed a hill after active primes such as A kid climbed every tree; hence, they did not simply repeat whether $a$ referred to one or potentially more than one entity. In contrast, they tended to repeat the interpretation of Every hiker climbed a hill after passive primes such as A tree was climbed by every kid. So, they repeated the use of an agentive noun with $a$ to refer to a single entity, even when this noun had a different grammatical function (i.e., subject versus oblique object) and was in a different linear position (i.e., first versus second NP). Participants therefore repeated mappings of scope to quantified thematic roles. Overall, these results support a semantic representation that encodes both quantificational and thematic information (but in which thematic roles are unordered). Critically, they do not support an account in which logical form (encoding quantification) constitutes a distinct level of representation between syntactic representation and final interpretation (e.g., May 1985).

We also assume that the semantic representation contains a specification of information structure. By information structure, we mean the way in which information is packaged with respect to the current context-for example, to reflect which information is known to the listener or is emphasized (e.g. Chafe 1976; Halliday 1967; Lambrecht 1994; Vallduvi 1992). In our account, information structure is specified with respect to thematic roles for example, that the Patient is emphasized (roughly corresponding to topic, theme, or given information, depending on theoretical framework).

In support of this claim, Vernice et al. (2012) showed that Dutch speakers repeated emphasis of particular thematic roles across sentences in the absence of syntactic or lexical repetition. They were more likely to produce passives with Patient-Agent order, which emphasized the Patient (e.g., Het meisje wordt overspoeld door de golf, "The girl is being soaked by the wave"), after Patientemphasis $W H$-cleft sentences with Agent-Patient order (Degene die hij slaat is de cowboy, "The one who he is hitting is the cowboy") than after Agent-emphasis WHcleft sentences with Patient-Agent order (Degene die hem slaat is de cowboy, "The one who is hitting him is the cowboy"). These results further support a representation containing unordered thematic roles and imply that these roles are specified with respect to information structure (see also Bernolet et al. 2009).

In all of these studies, priming occurred between sentences that involved different entities and/or different predicates, implying that the relevant representations were abstracted over these elements. Other priming evidence similarly supports a semantic representation framed in terms of abstract predicates, event components, and entities (Bunger et al. 2013; Raffray et al. 2014; see sect. 2.4).

\subsection{Structural representations and the lexicon}

So far, we have been concerned with characterizing the nature of syntactic and semantic representations, based on evidence of priming between sentences that share different aspects of structure in the absence of lexical repetition. These results provide evidence for at least some abstract representation of both syntactic and semantic structure. Additionally, however, a particularly robust finding is that various types of structural priming are 
enhanced considerably by repetition of the head of the local tree (the so-called lexical boost; e.g., Branigan et al. 2000; Cleland \& Pickering 2003; Hartsuiker et al. 2008; Pickering \& Branigan 1998). ${ }^{7}$ Both the existence of abstract priming and the lexical boost are informative about the lexical basis for linguistic representation.

Abstract syntactic priming provides evidence for a representation of syntax that is independent of lexical representation. The existence of priming between, say, Give the woman a book and Send the girl a letter indicates that the representation of grammatical information (here, about the DO structure) cannot be localized entirely to specific lexical entries. This is incompatible with one interpretation of lexicalist theories such as categorial grammars (Steedman 2000) and HPSG (Pollard \& Sag 1994). Such theories assume a few very general rules (e.g., function application, function composition, function substitution; Steedman 1987), but such rules cannot be the locus of abstract priming, because the same rules are applied across alternations such as DO and PO. To explain abstract priming, lexicalist theories must assume that the syntactic representations (e.g., VP/NP/NP in categorial grammar) are shared across lexical entries. Similarly, the occurrence of abstract semantic priming (e.g., emphasizing the Patient or producing coerced structures; Raffray et al. 2014; Vernice et al. 2012) implies that such information is not purely localized to lexical entries.

The existence of the lexical boost, however, also argues against an extreme structuralist account in which lexical information is not part of the central syntactic component for example, an account in which lexical entries are merely "slotted in" to a representation derived entirely from abstract (lexically unspecified) syntactic well-formedness constraints. Thus, there must be a representation that encodes a binding between constituent structure and the lemma (syntactic component) of the lexical entry for the head. For the sentence The man gives the book to the woman, this representation is $[\mathrm{V}[$ give $] \mathrm{NP} \mathrm{PP}] \mathrm{VP}$, where give is a lemma and not a complete lexical entry that additionally encodes semantic and phonological information. Importantly, the binding between $\mathrm{V}$ and the lemma give is the same type of binding that connects representations at different levels of structure (e.g., the syntactic and semantic representations associated with The man gives the book to the woman), rather than the links that connect components of the syntactic representation itself (e.g., linking VP and V). Repetition of the lemma and the syntactic well-formedness constraint that licenses the constituent structure (e.g., give and VP $\rightarrow \mathrm{V}$ NP PP) then leads to an enhanced priming effect. ${ }^{8}$

This account accords with the finding that the lexical boost appears to be due to repetition of a particular lemma (e.g., give) rather than a lemma that is instantiated for particular feature values (e.g., give $[+\mathrm{SING},+\mathrm{PRES}$, + PROG]). Pickering and Branigan (1998) found a lexical boost whenever the verb lemma was repeated, irrespective of whether the prime and target verbs shared tense, number, and aspect features (e.g., The racing driver was showing the torn overall to the mechanic yielded the same lexical boost as The racing driver showed the torn overall to the mechanic for the target The patient showed his wound to the doctor). Such results occur because the binding is between the constituent structure rule and a lexical entry without reference to features such as tense, but presumably with reference to syntactic category (to ensure that only well-formed bindings occur).

Little is known about priming of unbounded dependencies, and an interesting question is whether a constituent such as The book that the doctor gave to the patient would prime a PO, which would indicate whether a missing and an expressed NP differ in terms of a feature or a syntactic category. This distinction can be seen in two versions of Generalized Phrase Structure Grammar (GPSG). In both versions, The doctor gave to the patient is captured by VP/NP $\rightarrow \mathrm{V}$ NP/NP PP and NP/NP $\rightarrow \varnothing$. According to Gazdar et al. (1985; see also HPSG; Pollard \& Sag 1994), the slash-category /NP is simply a feature "missing NP." It is therefore similar to other features such as number (although it differs in having internal structure), and we have already noted that priming appears unaffected by feature differences. Thus, this account predicts that priming should occur in this case just as it does from a PO prime. But according to Gazdar (1981), slash categories differ at the categorical level from other categories. Hence, priming should be eliminated (or at least reduced) in this case. We know of no evidence that distinguishes these accounts.

Similar to syntactic priming, abstract semantic priming provides evidence for a representation of semantics that is independent of lexical representation. But there is also evidence for a lexical boost to semantic priming, even when the relevant elements are not present in the phonological representation. Raffray et al. (2014) found priming of coerced sentences when the events that the prime and target sentences described involved different entities and different coerced predicates (e.g., The celebrity began the champagne primed The clerk began the report; see sect. 2.4), implying the existence of semantic representations that were abstracted over these elements. However, they found a boost to priming when the coerced predicate was repeated between prime and target, even though the associated verb was not expressed: The celebrity began the champagne (coerced predicate: drink) was a stronger prime than The caretaker began the stairs (coerced predicate: sweep) for The banker began the tea (coerced predicate: drink). These results suggest the existence of bindings between lexical items (whether expressed or not) and semantic representations.

\subsection{Structural representations and their interfaces}

An account of structural representations also must specify mappings between levels of representation. Evidence from priming supports a range of mappings between information encoded in the semantic representation and information encoded in the syntactic representation: between thematic roles and grammatical functions, between thematic roles and word order, between animacy and syntactic structure, and between event structures and syntactic structures.

Cai et al. (2012) showed priming of mappings between thematic roles and grammatical functions. After hearing a Mandarin topicalized PO such as Naben shu niuzai song le gei shuishou, "The book, the cowboy gave [it] to the sailor," participants tended to produce POs (e.g., Jingcha song-le yiding maozi gei shibing; "The policeman gave a hat to the soldier"), in which the same thematic roles were mapped to the same grammatical functions (Theme 
Branigan and Pickering: An experimental approach to linguistic representation

to direct object and Recipient to oblique object) but different word order positions.

They also showed priming between thematic roles and word order: Participants also tended to produce POs (with Theme-Recipient order) after hearing a topicalized DO (which also has Theme-Recipient order; e.g., Naben shu niuzai song-gei le shuishou, "The book, the cowboy gave the sailor [it]"). Köhne et al. (2014) similarly showed that German participants tended to produce sentences with Theme-Recipient order following a prime with Theme-Recipient order (e.g., Der Mann verspricht die Putzhilfe der Ehefrau, "The man promises the cleaning woman the wife"). Additionally, Chang et al. (2003) found priming that was compatible with thematic-function mappings or thematic-order mappings. ${ }^{9}$ Bock et al. (1992) found that participants were more likely to produce descriptions in which an animate entity was a sentenceinitial subject (e.g., The boy is woken by the alarm clock) after reading and repeating sentences with an animate sentence-initial subject (Five people carried the boat, or Five people were carried by the boat) than an inanimate sentence-initial subject (The boat carried five people, or The boat was carried by five people). These results are compatible with priming of animacy-function or animacy-order mappings. However, other research has not found priming of animacy to syntactic structure mappings (Bernolet et al. 2009; Carminati et al. 2008; Huang et al. 2016).

Bunger et al. (2013) and Raffray et al. (2014) showed priming of mappings between components of event structures and syntactic structures. The former researchers demonstrated that speakers repeated mappings of components of motion events to syntactic structure. Participants who had read sentences in which information about the manner of a motion event was mapped onto the sentence-initial subject of the sentence (e.g., The zebra on the motorcycle entered the garage) were more likely to produce descriptions in which information about the manner of an unrelated event was similarly encoded in the sentence-initial subject (e.g., The driver is going into the cave) than participants in a control condition (who were not exposed to primes).

Raffray et al. (2014) investigated utterances expressing complex events in which speakers had a choice of how to map a complex event (e.g., the clerk beginning to read the report) onto syntactic structure. Specifically, the complex event involved three semantic elements: an event lacking a (subordinate) event (the clerk beginning); an event lacking an entity (the clerk reading); and an entity (the report). Speakers could map these semantic elements to two or three syntactic elements in the VP (i.e., V NP: began the report; or V V-ing NP: began reading the report). They were more likely to produce sentences such as The clerk began the report after sentences that similarly involved mappings to two syntactic elements (e.g., The celebrity began the champagne) than after sentences that expressed the same meaning (e.g., The celebrity began drinking the champagne) or used the same syntactic structure (e.g., The celebrity began the speech) but did not involve the same mappings. In conclusion, priming can uncover the relationship between misaligned syntactic and semantic representations, just as it can uncover the nature of syntactic and semantic representations themselves.

\section{Implications and predictions}

Section 2 discussed the implications of research on structural priming for many aspects of linguistic representation in adult native speakers. We now consider how our proposals relate to current theoretical linguistic frameworks. We then consider priming in bilingualism as a means of understanding structural representations across languages, and priming in children as a means of understanding structural representations during language development. We conclude by addressing broader implications and predictions of our proposals.

\subsection{Implications for linguistic theory}

We have argued that structural priming supports separate representations encoding semantic, syntactic, and phonological information. The single semantic level includes quantificational, information-structural, and thematic information, including information pertaining to elements that are not overtly expressed. The single syntactic level is specified in terms of grammatical categories (and does not include semantic, lexical, or phonological information). It captures local relations specifying linear order and hierarchical relations. It represents some missing elements, but there is no syntactic movement.

Our account is therefore incompatible with "mainstream generative grammar" (see Culicover \& Jackendoff 2005) the framework derived from early transformational grammar (Chomsky 1965) via Government and Binding Theory (Chomsky 1981) and the Minimalist Program (Chomsky 1995). This framework assumes that the generative capacity of language is associated strictly with the grammar. An initially abstract syntactic structure is altered sequentially through movement of elements (transformations). The resulting surface syntactic structure forms the input into both Logical Form (a "covert" level of syntactic representation that interfaces with semantic representations encoding sentence meaning) and Phonetic Form (which is concerned with sound-based aspects of the sentence).

The assumption of autonomous syntax, into which phonological content is subsequently inserted, fits with evidence of priming between sentences without shared lexical content (e.g., Bock 1989). The assumption that speakers may represent syntactically some elements that they do not utter fits with evidence that sentences with missing arguments prime sentences without missing arguments (Cai et al. 2015). But in other respects, mainstream generative grammar is incompatible with priming evidence about linguistic representation. Most fundamentally, priming studies provide no evidence for movement or a wide range of associated empty elements (e.g., traces, copies, or multiply dominated elements).

The clearest example involves passive sentences. Under a mainstream generative account, passives involve movement of the underlying object to subject position in the surface structure (leaving an NP trace or equivalent), whereas intransitive (active) locatives do not. Hence, the two sentence types involve very different representations. The mainstream account is therefore incompatible with evidence that intransitive locatives prime passives (Bock \& Loebell 1990) and that unergatives prime unaccusatives (Flett 2006). For similar reasons, it is inconsistent with 
Branigan and Pickering: An experimental approach to linguistic representation

evidence that transitive locatives prime POs (Bock \& Loebell 1990), and that POs and DOs prime light-verb sentences and vice versa (Wittenberg 2014). The assumption of a syntactic level of Logical Form (i.e., without specifications of meaning) is also incompatible with priming evidence for abstract semantic representations that specify quantifier scope (Chemla \& Bott 2015; Raffray \& Pickering 2010). Overall, the findings from structural priming do not support mainstream generative grammar.

Our account is more compatible with a broad range of alternative frameworks that eschew syntactocentrism and instead assume nondirectional and constraint-based generative capacities (i.e., specifying well-formed structures) that do not involve movement and in which syntactic structure is shallow and not limited to binary branching. Such frameworks include the Parallel Architecture (Culicover \& Jackendoff 2005; Jackendoff 2002), HPSG (Pollard \& Sag 1994), and Construction Grammar (Goldberg 1995). ${ }^{10}$

We focus here on the Parallel Architecture (Culicover \& Jackendoff 2005; Jackendoff 2002); see Jackendoff (2007) for an accessible and psycholinguistically oriented discussion. This framework assumes separate generative capacities for semantics, syntax, and phonology, and proposes that they are linked via interfaces, or mappings, that involve input from the lexicon. So the girl was chased by the dog might have the syntactic representation $\mathrm{S}[\mathrm{NP}[$ Det N]VP[Aux V PP], the semantic representation CHASED[DOG, GIRL] ${ }_{[\text {TOPIC] }}{ }^{11}$ and the phonological

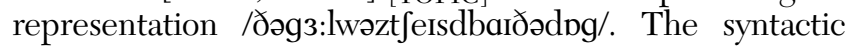
representation occurs through combination of "constraints" (stored fragments of structure) such as S[NP VP] and NP[Det N]. Culicover and Jackendoff (2005) also proposed a further tier of syntactic structure that captures grammatical function information associated with the ordering of NP arguments. Lexical entries comprise constraints (again, stored fragments of structure) such as DEF - Det - /ð॰ / and GIRL - N - /g3:I/ that play a role in the composition of sentence structure. They act as interface rules constraining relations between semantic, syntactic, and phonological representations. Such constraints yield coindexation of elements at different linguistic levels in parallel - for example DEF1 GIRL $2, N P[D e t 1 ~ N 2]$, and / $/ 2 / 1 / \mathrm{g} 3: 1 / 2$ (with the indices indicating the links between representational levels). All linguistic representations (whether semantic/ syntactic/phonological or lexical) are stored in long-term memory.

In many respects, this account is compatible with priming evidence. The assumption that speakers and listeners access the same local syntactic constraints that are independent of semantics or phonology (e.g., VP[V NP PP] for a $\mathrm{PO})$ is consistent with abstract syntactic priming over local structures. Shallow syntactic structure and the associated assumption that many detailed distinctions are made in the semantics rather than syntax (and that there is no movement) are compatible with priming between intransitive locatives and passives. Association of a lexical entry with a syntactic constraint (e.g., linking the entry for give with the PO constraint) accounts for the lexical boost. The assumption of a grammatical function tier as part of syntactic structure is consistent with priming of thematic-function mappings. The assumptions of abstract semantic representations based on events, predicates, and entities, which may include elements not represented in the syntax, together with interface constraints between semantics and syntax, are compatible with priming of semantic-syntactic mappings in sentences involving complement coercion and motion events (Raffray et al. 2014; Bunger et al. 2013).

This account is less compatible with evidence about the relationship between hierarchical relations and word order. Priming evidence suggests that hierarchical relations and word order are encoded in a single representation, because sentences with the same hierarchical relations but different word orders do not prime each other (Pappert \& Pechmann 2014; Pickering et al. 2002). In contrast, Culicover and Jackendoff (2005) proposed that there are independent constraints on hierarchical relations (constituency) and word order, as in GPSG (Gazdar et al. 1985) and HPSG (Pollard \& Sag 1994). They argued that separating these constraints allows important generalizations - for example, about regularities of phrasal ordering that are independent of hierarchical structure (e.g., about head position). Though these generalizations may be important, priming suggests that they do not reflect the representations used in language processing (see discussion in sect. 1.1).

Additionally, the Parallel Architecture account assumes that thematic structure, quantification, and information structure involve different tiers within semantics. Current priming evidence supports semantic representations that are specified for thematic roles in conjunction with quantification (Raffray \& Pickering 2010; priming of patients taking wide scope) and information structure (Vernice et al. 2012; priming of patients receiving emphasis). However, it does not discriminate whether these constitute one integrated semantic representation (as we have assumed) or multiple semantic representations for thematic roles, quantification, and information structure that are linked to each other (as in the Parallel Architecture account). Further research might distinguish these accounts by investigating whether priming involving two semantic components (e.g., quantification and information structure) is independent of another component (e.g., thematic roles).

More generally, structural priming has implications for linguistic theory in offering a means of adjudicating between alternative analyses that cannot be determined using other methods. For example, it may be able to resolve long-standing debates about the appropriate representation of English small-clause structures (e.g., He called the boy a liar, for which acceptability judgments support both a structure in which the boy and a liar do not form a constituent, and a structure in which they do; see Matthews 2007): Under the former account, a sentence such as He called the boy a liar should prime a sentence such as The doctor gave the pharmacist the pills, whereas under the latter account it should not. Similarly, it could resolve the ongoing controversy about Chinese bei-passives (e.g., Nashan de men bei niuzai chuai-huai-le, "That door by the cowboy was kicked in," for which acceptability judgments and constituency tests support both an analysis in which bei heads a prepositional phrase, and an analysis in which it heads a verb phrase; see Huang et al. 2009).

\subsection{Structural priming and representation across languages}

Our account is based on evidence from a range of languages with different characteristics (e.g., English, German, 
Mandarin, Basque). Importantly, structural priming occurs in all languages that have been investigated, including American Sign Language (Hall et al. 2014), and appears to exert similar effects. Moreover, priming evidence supports very similar representations for structures across languages. For example, Mandarin (a language unrelated to English) has an alternation that appears similar to the English PO/DO alternation, and Cai et al. (2011) found very similar priming as in English, with a comparable magnitude of priming and lexical boost. Likewise, evidence from Basque (a language with ergative properties) supports syntactic representations that, like those found in English, are independent of lexical, thematic, and morphological content (Santesteban et al. 2015). Evidence from typologically distinct languages therefore suggests that our account is not restricted to a small range of Western Indo-European languages with quite specific characteristics.

Many studies have shown strong priming in non-native speakers, even for structures that do not exist in their native language, and that priming has similar characteristics in natives and non-natives (e.g., occurring for the same constructions, and demonstrating the lexical boost; Cai et al. 2011; Flett et al. 2013; Kantola \& van Gompel 2011; Salamoura \& Williams 2006; Schoonbaert et al. 2007). Current evidence therefore suggests that linguistic representation is similar for natives and non-natives. Of course, it remains possible that native and non-native linguistic representations differ in subtle ways (e.g., in relation to unbounded dependencies; Clahsen \& Felser 2006).

Strikingly, structural priming occurs between languages, with effects often similar to those within languages. It occurs between many pairs of languages with differing degrees of similarity (e.g., German and English: Loebell \& Bock 2003; Dutch and English: Bernolet et al. 2009; Spanish and English: Hartsuiker et al. 2004; Korean and English: Shin \& Christianson 2009; Mandarin and Cantonese: Cai et al. 2011; Greek and English: Salamoura \& Williams 2007). These studies, of course, demonstrate abstract structural priming: The words are different across languages. But more interestingly, they imply that bilinguals not only use a common representational vocabulary across languages, but also the same structural representations where possible (and these representations are the same as those of monolinguals). One relevant restriction on structure sharing is word order: Between-language priming is reduced or eliminated when the structures have different word orders across languages (e.g., English: the shark that is red vs. Dutch: de haai die rood is, "the shark that red is"; Bernolet et al. 2007). This restriction follows from our assumption that syntactic representations are specified for both hierarchical and linear relations. Other studies of between-language priming support our claims that semantic representations encode thematic information and information structure (e.g., Bernolet et al. 2009; Fleischer et al. 2012).

More speculatively, structural priming might allow researchers to detect linguistic universals that are accessible in adult speakers (i.e., not just as an initial state that disappears during development). For example, priming has not been demonstrated with agglutinative languages. Our account assumes abstract syntactic structure, independent of lexical or morphological content, and hence that priming will occur between examples of the same structure in which the verb involves considerable morphological differences. For instance, a sentence with an NP PP V syntactic representation would prime another sentence with the same representation even if the verb contained many different morphemes, as is possible in an agglutinative language (e.g., Turkish). But if such priming does not occur (or is affected by morphological overlap), it would suggest that syntactic representations are morphosyntactically specified in such languages, so that there is no single well-formedness constraint VP à NP PP V, but rather different ones depending on the form of the verb.

Another possibility is that constituent structure is not universal (e.g., Evans \& Levinson 2009). For example, some researchers have argued that some languages (e.g., Walpiri) are nonconfigurational and do not have hierarchical constituent structure (Hale 1983; Austin \& Bresnan 1996). If so, they should not give rise to constituent structure priming within or between languages (though careful comparisons are clearly needed to control for other sources of priming such as thematic order priming).

We propose that a thorough analysis of priming across a full range of languages (e.g., agglutinative and isolating languages, languages with ergative characteristics, nonconfigurational languages, sign languages) is necessary to determine the extent to which our account holds universally, or whether different types of languages involve different representational structures. If our account does not hold universally, then it may still be possible to establish that some properties are universal and some vary across languages. For example, all languages might involve a distinction between semantic and syntactic representations, but in some languages syntactic representations might include "missing" elements and in some languages they might not. Priming, therefore, might allow us to develop a cognitive representational approach to language typology.

\subsection{Structural priming and language development}

Research on language development has recognized the importance of priming as a means of investigating structural representation, perhaps more strongly than research on adult language (Bencini \& Valian 2008; Messenger et al. (2012b); Rowland et al. 2012; Savage et al. 2003). Structural priming occurs in children across age groups (e.g., 3-year-olds: Bencini \& Valian 2008; 6- and 9-year-olds: Messenger et al. (2012a); 3- to 4-year-olds and 5- to 6year-olds: Rowland et al. 2012; 3-, 4-, and 6-year-olds: Savage et al. 2003; 7 - to 8- and 11- to 12-year-olds: van Beijsterveldt \& van Hell 2009), in comprehension as well as production (4-year-olds: Thothathiri \& Snedeker 2008b). It occurs in children in different languages (e.g., Englishspeaking 4- to 5-year-olds: Huttenlocher et al. 2004; Spanish-speaking 4- and 5-year-olds: Gámez et al. 2009; Russian-speaking 5- to 6-year-olds: Vasilyeva \& Waterfall 2012), and populations, including bilinguals (between languages; 5- to 6-year-olds: Vasilyeva et al. 2010), deaf children (11- to 12-year-olds: van Beijsterveldt \& van Hell 2009), children with Specific Language Impairment (4- to 6-year-olds: Garraffa et al. 2015; Leonard 2000; Miller \& Deevy 2006; 6- to 7-year-olds: Riches 2012), and children with an Autistic Spectrum Disorder (8- to 13-year-olds: Allen et al. 2011; Hopkins et al. 2016). Of course, some of these children cannot make grammaticality or acceptability judgments, so it simply would not be possible to 
investigate their structural representations if researchers relied on these methods.

Evidence from these studies suggests that, from a relatively young age, children's structural representations are similar to adults'. Like adults, 3- and 4-year-olds appear to have abstract syntactic representations that are not specified for lexical or thematic content (e.g., Bencini \& Valian 2008; Huttenlocher et al. 2004; Messenger et al. (2012b); Rowland et al. 2012). Rowland et al. showed that they tended to produce DOs after hearing and repeating DOs involving different nouns and verbs (e.g., Prime: The king brought the queen a puppy - Target: Dora gave Boots a rabbit). Messenger et al. (2012b) showed they were primed to produce passives involving Patient/Agent thematic roles (e.g., The witch was hugged by the cat) to the same extent when the prime involved Experiencer/ Theme roles (e.g., The girl was shocked by the tiger) and Theme/Experiencer roles (e.g., The girl was ignored by the tiger). There is some evidence of a lexical boost in children (3- to 4-year-olds: Branigan \& McLean 2016; 7 - to 8year-olds: van Beijsterveldt \& van Hell 2009). Interestingly, there is no evidence of a stronger lexical boost in young children compared to older children and adults (3- to 4year-olds: Peter et al. 2015; Rowland et al. 2012), as might be expected on an account in which early grammars involve "islands" of information associated with individual verbs, that is, partly lexicalized syntactic structures (Tomasello 1992). These priming studies therefore contribute important evidence to the debate about the extent to which children's early structural representations are abstract versus lexically specified (e.g., Fisher 2001; Goldberg 2006; Pinker 1989; Tomasello (2003a)).

Importantly, structural priming experiments also have provided evidence to discriminate specific theoretical linguistic accounts (motivated by error and frequency data) of young children's syntactic representations. Messenger et al. (2012a) demonstration of priming between ExperiencerTheme and Agent-Patient passive sentences provided evidence that 3- to 4-year-olds have an abstract representation of passive structure that is not semantically restricted (contra Maratsos et al. 1985). Likewise, Messenger et al.'s (2011) demonstration of priming between short passives and full passives suggests that 3-to 4-year-olds do not represent short passives in a distinct way from full passives (for example, as an adjectival phrase; Borer \& Wexler 1987; Horgan 1976).

Children's semantic representations also appear similar to those of adults. For example, Gámez et al. (2009) and Vasilyeva and Waterfall (2012) showed priming of thematic emphasis in Spanish-speaking 4- to 5-year-olds and Russian-speaking 5- to 6-year-olds (with passive structures priming patient-emphasized structures), suggesting that children have a thematically specified representation of information structure. Viau et al. (2010) found priming of abstract quantified representations, with respect to the scope of negation, in 4-year-olds' comprehension. Children were more likely to adopt a negation-wide interpretation of Every horse didn't jump a fence after hearing a sentence with a negation-wide interpretation than after a sentence with negation-narrow interpretation, even when the prime differed in syntax and quantifier order (e.g., Not every horse jumped over a pig). These findings all suggest that, at least from age 3 , children and adults have similar representational structures at each level, and similar interfaces between levels. However, it is clearly necessary to test further structures, as well as younger children if possible.

\subsection{Further implications}

We have argued that the method of structural priming is informative about linguistic representation with reference to evidence from not only monolingual adults, but also bilingual adults and children. Other relevant evidence relates to atypical populations, including demonstrations of structural priming in aphasia (Hartsuiker \& Kolk 1998; Saffran \& Martin 1997), Specific Language Impairment (Garraffa et al. 2015; Leonard et al. 2000), and amnesia (Ferreira et al. 2008). For example, aphasic speakers may produce passives (although often containing morphological errors) after repeating unrelated passives, despite not producing such structures spontaneously. Such findings suggest that structural representations may be intact even if not evinced in patients' spontaneous language behavior; these findings also may be relevant to therapy. More theoretically, priming evidence can be used to determine the structure of linguistic representations in language pathologies. Additionally, the neural underpinnings of priming are not well understood (although see Menenti et al. 2011; Noppeney \& Price 2004; Segaert et al. 2012; Segaert et al. 2013), but priming is likely to be informative about neurolinguistic representation.

We further propose that structural priming similarly can be used to investigate other aspects of cognition involving structured representations. These may include representations of the results of complex human activities involving domains such as music, mathematics, and artificial languages. In such cases, the representations may of course be derivative of linguistic representations (though it is also possible that they developed independently). For example, Scheepers et al. (2011) showed that people tended to repeat their interpretation of complex arithmetical expressions that lacked brackets (in other words, copying the bracketing from prime to target) and, moreover, that language and arithmetic could prime each other. Similar priming occurred between language and music (van der Cavey \& Hartsuiker 2016). Another relevant domain is gesture, in which evidence suggests that people repeat gesture patterns ( $\mathrm{Mol}$ et al. 2012). However, there is no clear priming evidence about the structure of complex gestures expressing events (see Goldin-Meadow et al. 2008). Additionally, it may be possible to investigate priming of structured animal calls (Schlenker et al. 2014). In these cases, there is either little evidence about structure, or it is simply assumed that some standard representation (e.g., musical or mathematical notation) is adequate for explaining cognitive representations. Priming may be informative about these representations and, indeed, the relationships between such representations across domains.

Finally, we return to priming of comprehension: the tendency for comprehension to be affected by comprehension (or production) of previous utterances that share aspects of structure. We have not focused on it because the data are much more limited and less clearly established than priming of production (e.g., there are contradictions concerning when priming occurs without verb repetition; Arai et al. 2007; Thothathiri \& Snedeker 2008a), and because experimental conditions often differ extensively 
in both form and meaning (e.g., main clause vs. reduced relatives; see Traxler et al. 2014). ${ }^{12}$ But priming of comprehension occurs when prime and target differ primarily in form (e.g., active/passive, $\mathrm{PO} / \mathrm{DO}$ ), and the effects reveal shared processes with priming of production (Segaert et al. 2013). Priming in comprehension can be informative about the representation of structures in the absence of alternatives (i.e., when participants do not choose between alternative structures), in a way that appears hard to demonstrate in production. It also may be valuable for investigating populations whose ability to produce language is restricted (e.g., very young children, some aphasics). Importantly, we propose that priming in comprehension is likely to become a technique of similar importance to priming in production for determining linguistic representation. ${ }^{93}$

\section{Conclusion}

Many linguists assume that acceptability judgments are pretty much the only valid means of obtaining data that are informative about linguistic representation. Instead, we have argued that structural priming can provide a valid method with many advantages and have shown how experimental psychology (and not just traditional linguistics) can be informative about the nature of language. We have now reached the stage at which structural priming is a mature method that provides extensive evidence about representation. Thus, we have used that evidence to develop a general approach to linguistic representation. This account is largely but not entirely compatible with a parallel linguistic architecture (e.g., Culicover \& Jackendoff 2005), though the data support the existence of some empty elements in the syntactic representation. Structural priming provides evidence about linguistic representation that informs linguistic theory, processing accounts that are based on such theories, and claims about development and language universals. It is a method that truly has come of age and should help integrate linguistics and the psychology of language as part of the cognitive sciences of language.

\section{ACKNOWLEDGMENTS}

Holly Branigan was supported by a British Academy/Leverhulme Trust Senior Research Fellowship. This research was supported by the European Union's Seventh Framework Programme for research, technological development and demonstration under grant agreement no. 613465; the Economic and Social Research Council (grant number ESN0131151); and a Royal Society of Edinburgh/National Science Foundation of China research grant. We thank Caroline Heycock and Mante Nieuwland for helpful comments; all views expressed are our own.

\section{NOTES}

1. Judgments may be nonbinary, with sentences being judged more or less acceptable, most obviously when elicited using magnitude-estimation tasks (Bard et al.1996) or Likert scales, but even researchers who eschew these methods usually assume that some sentences are "questionable" or "marginal." However, these judgments still relate to set membership.

2. Priming effects can also be inhibitory (e.g., Goldinger et al. 1989), and speakers may avoid linguistic repetition on occasion (see Szmrecsanyi 2006). However, structural priming studies have focused so far on facilitatory effects.
3. Some models of language processing assume that the representations proposed by traditional linguistic theories are an approximation to statistical generalizations that emerge with experience (see Seidenberg 2007). If so, structural priming effects are informative about these generalizations. For example, the evidence that priming occurs between sentences with different lexical content implies that some such generalizations are not tied to particular words.

4. The historical division of labor means that priming experiments concerned with representational questions typically have investigated hypotheses generated on the basis of acceptability judgments. But priming experiments are not parasitic on acceptability judgments any more than any new scientific method is parasitic on an older method that addressed the same issues. Acceptability judgments are chronologically primary to priming experiments in the history of the language sciences but are not theoretically primary.

5. Scheepers (2003) found that, when people completed sentences such as The assistant announced the score of the candidate that, they tended to repeat whether they attached the modifier to the first or the second NP (e.g., was the highest vs. was the oldest). Another experiment ruled out a purely semantic explanation. Arguably, the sentence types involve the same set of contextfree phrase structure rules (in particular, an NP consists of an NP followed by a complementized sentence). One possible explanation is that priming may occur over larger elements of structure than strictly local trees. If so, people may represent frequent or important "chunks" of more global structure as well as local relations (see Culicover \& Jackendoff 2005). However, this explanation provides no evidence against the existence of locally defined representations (Branigan et al. 2006).

6. Griffin and Weinstein-Tull (2003) found that people were more likely to produce Alison wished the bad news to be a mistake (vs. Alison wished that the bad news was a mistake) after Rover begged his owner to be more generous with food than after The teaching assistant reported the exam to be too difficult. The primes have the same constituent order (NP V NP Vinf). They differ in semantics (report takes one argument [(the event[difficult(exam)]), yielding report[difficult(exam)]], whereas begged takes two (the entity owner and the event generous [owner]), yielding begged(owner, generous[owner])); but the two versions of the target have the same semantics, so this cannot be the locus of priming. A possible explanation is that priming takes place over a syntactic representation in which an argument can be represented twice. Thus, his owner is represented twice, corresponding to its semantic representation as an argument of begged and as an argument of generous, whereas the exam is represented once, as an argument of difficult. This explanation assumes that the syntactic representation includes missing elements. The authors, however, interpret the priming in terms of a mapping between semantic and syntactic representations, and we cannot distinguish the accounts.

7. The lexical boost is not solely due to semantic similarity between prime and target, though such similarity enhances priming (Cleland \& Pickering 2003): Cross-linguistic priming (see sect. 3.2) using translation-equivalent verbs is smaller than would be expected if the lexical boost resulted purely from semantic repetition (Bernolet et al. 2012; Cai et al. 2011; Schoonbaert et al. 2007).

8. For convenience, we use $\mathrm{X} \rightarrow \mathrm{Y} \mathrm{Z}$ to express declarative (non-directional) well-formedness constraints on representations.

9. We argued above that the lack of priming between sentences with V PP NP and V NP PP constituent order (Pappert \& Pechmann 2014; Pickering et al. 2002) supports a monostratal account of syntactic representation in accord with Cai, Pickering, and Branigan (2012), there is no effect of unordered constituent structure, and the thematic-order and thematic-function effects cancel each other out.

10. A challenge for Construction Grammar is the evidence that priming seems unaffected by whether prime and target involve 
the same construction (form-meaning pairing) or not. Thus, Konopka and Bock (2009) found equivalent priming within and between non-idioms (e.g., The graduating senior sent his application in) and idioms (e.g., The teenager shot his mouth off), which constitute different constructions in Construction Grammar. An explanation of such findings in terms of Construction Grammar would have to assume that the form component of constructions can be primed, and that priming takes place between different constructions that share form components to the same extent as it does within a construction. Hence, priming could not be used to support the existence of form-meaning pairings.

11. In Culicover and Jackendoff's (2005) account, information structure forms a separate tier of semantic representation from propositional structure.

12. Many studies demonstrate facilitation following repeated presentation of a construction - for example, reduced processing times for strong garden-path sentences (Fine et al. 2013) or marginally unacceptable sentences (Kaschak \& Glenberg 2004), and a higher likelihood of judging marginally unacceptable sentences as acceptable (Luka \& Barsalou 2005). But the relationship between such studies and structural priming studies involving individual prime-target pairs is unclear.

13. Priming may affect response times in production (Corley \& Scheepers 2002; Smith \& Wheeldon 2001), but current evidence overwhelmingly relates to structure choice.

\section{Open Peer Commentary}

\section{The limitations of structural priming are not the limits of linguistic theory}

\author{
doi:10.1017/S0140525X17000310, e283
}

\section{David Adger \\ Department of Linguistics, Queen Mary University of London, London E1 4NS, United Kingdom. \\ d.j.adger@qmul.ac.uk \\ http://www.davidadger.org}

Abstract: Structural priming is a useful technique for testing the predictions of linguistic theories, but one cannot conclude anything definitively about the shape of those theories from any particular methodology.

Branigan \& Pickering $(\mathrm{B} \& \mathrm{P})$ present a case that linguistic theory should pay heed to the results of structural priming studies. I can agree with this wholeheartedly. Since the pioneering work of Bock (1986), structural priming has provided interesting evidence for the construction of linguistic representations as part of the process of sentence generation and understanding. $\mathrm{B} \& \mathrm{P}$ are somewhat ambivalent on the question of whether linguistic theory should pay heed only to structural priming (as seems implied in the abstract) or simply add it to the repertoire of methodologies for testing the predictions of theories. If simply an addition to the repertoire, again, I am in thorough agreement.

However, B\&P draw unwarranted conclusions about the content of linguistic theory on the basis of their discussion of structural priming. B\&P mention Chomsky's (1981) point that one should look carefully at the experiment, as well as the theory, when faced with a negative result in an experimental test of a theory. They write that this has been taken as an excuse for theorists to ignore experiments. It is, rather, an injunction to think about what the implications of the result mean. Often, theory is built on extremely solid empirical premises, and if the experiment appears to disconfirm the theory, then one has to ask whether the problem lies with the premises, the theory, or the experimental design. In many cases, the experiment simply does not have enough sensitivity to overturn an otherwise solid empirical base and concomitant theoretical conclusions.

Let's take a hoary example:

1. We saw the boy with the telescope. As any Lin101 student will tell you, this is structurally ambiguous. There are numerous avenues for theoretically modelling this fact about (1). One approach would be to have some rule system that licenses two distinct representations: in one, a PP attaches to NP; in another, it attaches to VP. The hypothesized rule system makes predictions about the behaviour of other sentences. If a PP can attach to VP or NP, then this rule system predicts (2), (3), and (4) independently. The grammaticality of these sentences (and countless others, of course) is further evidence that what was hypothesized to explain the ambiguity is correct.

2. The boy with the telescope arrived.

3. We arrived with the telescope.

4. We saw the boy with the telescope with the telescope.

Combined with the unassailability of the basic facts, the theory's deductive complexity is what gives it its epistemic strength.

Now, let us look at what structural priming has to tell us about (1) and examples like it. In previous work, Branigan et al. (2005) showed that, when the PP attachment height is disambiguated by pictures presented to experimental subjects, those subjects will maintain the same PP attachment height for similarly structurally ambiguous new sentences - a case of structural priming in comprehension. There is a twist, however: Such priming takes place only when the verb is repeated. There is no significant effect when the newly presented sentence is structurally ambiguous in the same way but contains a different verb. So, what can we conclude from this? Because structural priming has no effect, the sentences presented with different verbs are not structurally ambiguous? Imagine no priming effect had been found at all. Would this mean the theory was wrong? No. It would mean only that structural priming was insufficiently sensitive. There is no legitimate inference from the failure of structural priming to claims about the content of the grammar.

For similar reasons, B\&P's arguments that mainstream generative linguistic theory is incompatible with evidence from structural priming do not go through: The problem is that structural priming is not sensitive enough to pick up on independently verifiable syntactic distinctions. B\&P motivate their claim by using, inter alia, the fact that unergative sentences (such as Lilly danced) prime unaccusative sentences (such as Lilly froze). Generative syntax takes these verbs to be in two distinct syntactic classes, with their subjects having distinct structural properties. The priming result looks incompatible with this claim, because it places them in the same class. There is linguistic evidence for this distinction internal to English, however: Unaccusatives lead to resultative readings for secondary predicates, while unergatives do not (compare Lilly froze solid with " Lilly danced tired, which has, at best, a depictive reading). There is also a great deal of cross linguistic evidence for the distinction, motivated by numerous syntactic differences, including the kind of auxiliary that the verb appears with, the possible positions for the subject noun phrase, and the behaviour of pronominal elements in clause structure (see Alexiadou et al. [2004]). There is also solid processing evidence for the distinction from different experimental paradigms: Friedmann et al. (2008), in an online cross-modal lexical priming experiment on native English speakers, showed priming effects attributable to the two hypothesized structural positions for subjects; Lee and Thompson (2011) showed that fixation differences in eyetracking are sensitive to the unergative/unaccusative distinction. Does the absence of a structural priming effect mean we should ignore linguistic, cross-linguistic, and psycholinguistic evidence? Does it mean that we therefore should 
adopt a theory in which no unergative/unaccusative distinction is made? No, because there is a simple explanation for the disparity in results. Structural priming is not sensitive enough to capture this syntactic distinction. B\&P's argument from structural priming against mainstream generative syntactic theory is too logically weak to carry the burden placed upon it.

$\mathrm{B} \& \mathrm{P}$ take the limitations of structural priming to tell us something about the shape of the grammar and the representations that it legitimates. There is no reason to think this, however. There is no argument that takes us from the particularities of any method to conclusions about the object being studied, and to think that there is is to confuse the nature of the world with our ways of trying to understand it.

\section{Horses for courses: When acceptability judgments are more suitable than structural priming (and vice versa)}

\author{
doi:10.1017/S0140525X17000322, e284
}

\section{Ben Ambridge}

Psychological Sciences, University of Liverpool, Liverpool, L69 7ZA, United Kingdom; ESRC International Centre for Language and Communicative Development (LuCiD).

ben.ambridge@liverpool.ac.uk

www.benambridge.com

\begin{abstract}
Although structural priming is often the most suitable paradigm, it sometimes misses effects that are detected by more sensitive acceptability-judgment tasks, thus yielding incorrect conclusions. For example, Branigan \& Pickering's (B\&P's) claim that "syntactic representations do not contain semantic information" (sect. 2.1, para. 2), while supported by structural-priming studies of the passive, is undermined by an acceptability-judgment study of this construction.
\end{abstract}

I agree with Branigan \& Pickering $(\mathrm{B} \& \mathrm{P})$ that structural priming is an excellent method for tapping into speakers' linguistic representations. I agree with B\&P that "researchers concerned with linguistic representations should not rely solely on [acceptability] judgments" (sect. 1.2, para. 10). I agree with B\&P that, frequently, "evidence from structural priming will converge with evidence from acceptability judgments and hence provide strong support for specific representational claims" (sect. 1.5, para. 2).

I do not agree with $\mathrm{B} \& \mathrm{P}$ that "where acceptability judgment and priming evidence do not converge, evidence from priming should be favored" (sect. 1.5, para. 2). Why? The most suitable method for linguistic research depends on exactly what we want to know. Sometimes, structural priming is indeed the best choice: for example, when we want to know whether particular forms share some underlying representation. Sometimes, forced-choice comprehension is the best choice: for example, when we want to know if children understand the meaning of word order or case marking.

And sometimes, an acceptability judgment paradigm is the best choice: for example, when we want to know which of two similar forms is more consistent with adult speakers' underlying grammatical representations (e.g., "The funny clown giggled Bart vs *The funny clown laughed Bart). This really can be determined only using a Likert-scale-type judgment. Structural priming is all but useless here, because no adult native speaker of English is going to produce either sentence, no matter how much you prime her. The broader problem is that structural priming yields a binary outcome measure: You're primed, or you're not; you produce the sentence, or you don't. In contrast, acceptability judgments, if set up to do so, yield a continuous outcome measure. Crucially, the use of a relatively insensitive binary measure over a much more sensitive continuous measure can lead to erroneous conclusions regarding representation. Here's a case study.
B\&P argue that "syntactic representations do not contain semantic information" (sect. 2.1, para. 2) and that "adults ... appear to have abstract syntactic representations that are not specified for lexical or thematic content." A crucial piece of evidence for this claim is a series of structural priming studies (Messenger et al. 2012a; $2012 \mathrm{~b}$ ) in which passives with agent-patient, theme-experiencer and experiencer-theme verbs (e.g., The girl is being licked/scared/ ignored by the cow) were equally effective at priming production of other passives. Hence - B\&P's argument goes - adults' representation of the passive (a syntactic representation) does not care about the identity of the verb (semantic and/or lexical content) or, indeed, its thematic content (i.e., the way its syntactic arguments are mapped onto the semantic roles agent, patient, experiencer and theme).

This conclusion is incorrect, however. In one of my own studies (sorry!) based closely on Messenger et al. (2012b), we asked adults to rate these types of sentences on a 5-point scale (Ambridge et al. 2016). Passives with experiencer-theme verbs (e.g., ignore) were rated as less acceptable than passives with agent-patient (e.g., lick) and theme-experiencer verbs (e.g., scare), a pattern that did not hold for actives. This suggests that adults' representation of the passive construction is not purely syntactic but contains lexical/semantic/thematic-role information such that "[B] (mapped onto the surface subject [of a passive]) is in a state or circumstance characterized by [A] (mapped onto the by-object or an understood argument) having acted upon it" (Pinker et al. 1987, p. 249). In other words, the more the first NP is affected by the action, the better the passive-which also explains why three hours can't be lasted by a film or five people slept by a tent (cf. The film lasted three hours; This tent sleeps five people).

Why did we find lexical/semantic/thematic-role differences between passive sentences, but Messenger et al. (2012b) did not? Simple: We used a continuous DV ("How good is this sentence on a 5-point scale?"), while they used a less sensitive binary DV ("Does this sentence prime another passive?). This does not mean that acceptability judgments are always a better choice than structural priming; sometimes, the reverse is true. Horses for courses.

\section{ACKNOWLEDGMENT}

Ben Ambridge is Professor in the International Centre for Language and Communicative Development ( $\mathrm{LuCiD})$ at The University of Liverpool. The support of the Economic and Social Research Council [ES/ L008955/1] is gratefully acknowledged.

\section{Microscopic and macroscopic approaches to the mental representations of second languages}

\section{doi:10.1017/S0140525X17000334, e285}

\section{Zhenguang G. Cai ${ }^{\mathrm{a}}$ and Haitao Liu ${ }^{\mathrm{b}, \mathrm{c}}$

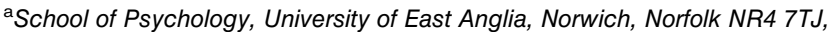

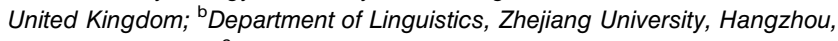 CN-310058, China; 'Centre for Linguistics and Applied Linguistics, Guangdong University of Foreign Studies, Guangzhou, CN-510420, China. zhenguangcai@gmail.com Ihtzju@gmail.com sites.google.com/site/zhenguangcai/ \\ person.zju.edu.cn/en/lht}

Abstract: With a particular reference to second language (L2), we discuss (1) how structural priming can be used to tap into L2 representations and their relationships with first and target language representations; and (2) how complex networks additionally can be used to reveal the global and local patterning of L2 linguistic features and L2 developmental trajectories.

One can draw methodological parallels between linguistics and astronomy in their development as scientific disciplines. Millennia of naked-eye astronomical observations (e.g., movement of stars) had advanced our understanding of the universe, but modern 
astronomy did not take off until the invention of the telescopes that allowed close (microscopic) examination of celestial bodies and mathematical formulations (e.g., gravitational attraction) that provided tools for large-scale (macroscopic) characterisation of stellar and galactic systems. Linguistics follows a similar track. Whereas decades, if not centuries, of intuitional data have offered deep insights into language, the availability of experimental and mathematical techniques has now afforded the opportunity to uncover the mental representations underlying language and to verify and advance existing intuition-based theories. In this sense, we applaud Branigan \& Pickering (B\&P) for their proposal to use structural priming to map out linguistic representations. In addition, we propose that mathematical tools such as complex networks allow us to unveil the laws governing the macroscopic patterning of language. In this commentary, we discuss how structural priming and complex networks can be used complementarily to approach our linguistic knowledge, with a particular reference to second language (L2) in adult learners.

The dominant view among L2 researchers is that learners' linguistic knowledge can be inferred from their grammaticality intuitions (using acceptability judgments), whereas psycholinguistic methods (e.g., eyetracking) merely capture language comprehension and production processes (e.g., VanPatten 2014). However, as argued convincingly by $\mathrm{B} \& \mathrm{P}$, acceptability judgement is a crude method susceptible to plausibility and processibility confounds and decisional biases (all the more so when judgements come for learners, as in L2 research). Further insight into L2 representations therefore requires more refined experimental methods. As B\&P proposed, structural priming fits the niche as an implicit method that reflects representational similarity between the prime and the target. Indeed, much research in the past decade has employed structural priming to show that learners have integrated representations for similar constructions between their first language (L1) and L2 (Chen et al. 2013; Hartsuiker et al. 2004, 2016). Such integrated representations may reflect a strategy for efficient learning: When possible, an L2 construction co-opts an existing L1 representation. This, in turn, may account for the pervasiveness of L1 transfer in L2 (Chan 2004; Eubank 1996).

Apart from revealing L1-L2 relationship, structural priming can be used to reveal the extent to which an L2 representation can be identified with its corresponding representation in the target language (TL). L2 learners, it was claimed, do not have as fully fledged syntactic representations as native speakers do (Clahsen \& Felser 2006). Such a claim can be tested using structural priming within L2. For example, Cai et al. (2012) showed that a dative sentence with a missing argument behaves similarly to its corresponding full-form sentence in priming dative sentences, suggesting that native speakers of Mandarin syntactically represent the missing argument even though it is not phonologically realised. If L2 speakers do not develop the same level of representational sophistication for missing-argument sentences, one would then expect reduced priming from a missing-argument dative compared to its full-form counterpart in L2. In addition, structural priming can be used to map out the developmental trajectory for syntactic constructions (McDonough \& Mackey 2006). It has been proposed that L2 learners initially develop item-specific structural representations and gradually transit to more abstract representations, as children do (Tomasello 2000). If so, a syntactic structure should exhibit only lexically driven structural priming when initially learned and lexicalindependent priming at a later stage (Rowland et al. 2012).

Structural priming, however, is less useful when it comes to evaluating the L2 system at a macroscopic level (e.g., to what extent L2 syntax or lexicon resembles its TL or L1 counterpart), an issue that research using complex networks attempts to address. Network science treats language as a complex system at each level (e.g., lexicon, syntax, phonology), consisting of interconnected elements (e.g., concepts, words, phonemes) (see Fig. 1 for an example). Complex networks allow for the characterisations of these interconnections that are indicative of global and local patterning of linguistic elements (Cong \& Liu 2014; Mehler et al.

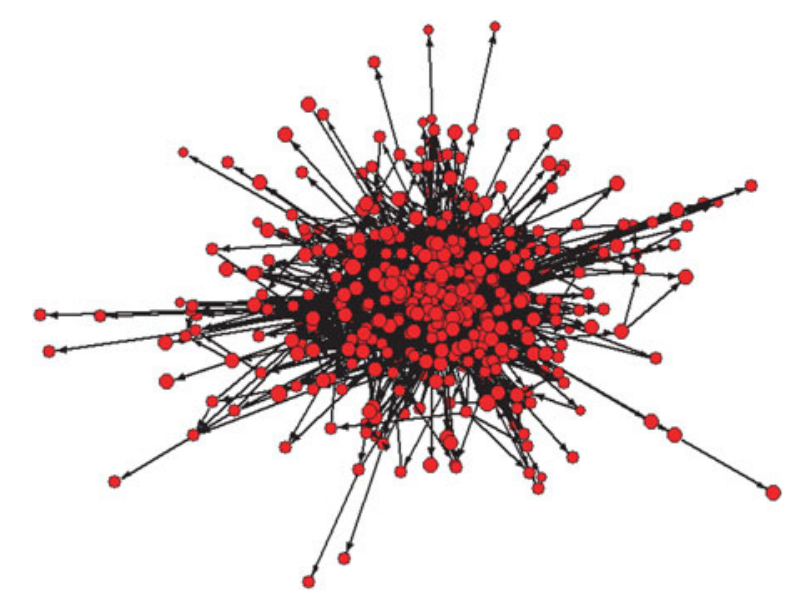

Figure 1 (Cai \& Liu). A syntactic network based on a 5000-word L2 text. Networks like this can be compared in terms of their parameters at different learning stages to reveal developmental trajectories, or the dynamic relations between L2 and L1 and between L2 and TL.

2015). For example, one can examine the average distance among syntactic relations to determine the complexity of syntax, or examine the interconnectivity among lexical concepts to determine the structure of the lexico-semantic representations. Indeed, complex networks have been applied to phonology (Siew \& Vitevitch 2016), morphology (Čech \& Mačutek 2009), lexico-semantics (Steyvers \& Tenenbaum 2005), syntax (Ferrer i Cancho et al. 2004), semantics (Liu 2009), language typology (Liu \& Li 2010) and L1 acquisition (Ke \& Yao 2008; Corominas-Murtra et al. 2009). In theory, we can apply similar networks to L2 to explore how L2 is organized at these different levels. Borodkin et al. (2016) and Wilks \& Meara (2002), for example, used complex networks to show that lexico-semantic organization is less optimal (e.g., lexical concepts are clustered to a lesser extent into subcategories such as vegetables and fruits) in L2 than in L1. Apart from examining static patterns in L2, future work can also explore L2 as a dynamic system by constructing networks (e.g., of syntactic relations) at different time points of L2 learning; by examining the changes in the network parameters, one can explore the development of a particular L2 feature (e.g., syntax or morphology). By constructing similar cross-sectional networks, one also can map out the dynamic trajectory of L2 approximation to TL and L2 detachment from L1.

All in all, we believe the time has come to use structural priming to examine L2 mental representations, and complex networks to extract the underlying patterning of L2 linguistic features. Such a convergent approach, making use of both microscopic and macroscopic analysis of linguistic features, is important for constructing a theory of L2 representations and L2 acquisition, and indeed, a theory of linguistic knowledge in general.

ACKNOWLEDGMENT

Preparation of this manuscript was supported by an ESRC grant to Zhenguang G. Cai. (S/L010224/1).

\section{Don't shoot the giant whose shoulders we are standing on}

\author{
doi:10.1017/S0140525X17000346, e286
}

Jan P. de Ruiter ${ }^{\mathrm{a}}$ and Laura E. de Ruiter ${ }^{\mathrm{b}}$

${ }^{\mathrm{a}}$ Department of Psychology, Tufts University, Medford, MA 02155; ' ${ }^{\mathrm{L}} \mathrm{CuCiD}$, School of Health Sciences, University of Manchester, M13 9PL Manchester, United Kingdom. 
Commentary/Branigan and Pickering: An experimental approach to linguistic representation

\section{jp.deruiter@tufts.edu}

https://ase.tufts.edu/psychology/people/de_ruiter/

laura.deruiter@manchester.ac.uk

http://www.lucid.ac.uk/who-we-are/our-people/researchers/laura-de-

ruiter/

Abstract: Structural priming is a sufficient but not a necessary condition for proving the existence of representations. Absence of evidence is not evidence of absence. Cognitive science relies on the legitimacy of positing representations and processes without "proving" every component. Also, psycholinguistics relies on other methods, including acceptability judgments, to find the materials for priming experiments in the first place.

We wholeheartedly agree with the authors about one thing: The demonstration of priming of a hypothesized representation is sufficient evidence for the existence of that representation. This is true even if this demonstration is possible only in controlled lab experiments. (In natural conversation, in fact, people do not have the "tendency to repeat their own and others' structural choices" (target article, abstract) as Healey et al. [2014] have demonstrated convincingly). Using structural priming as a necessary condition for the existence of representations "of any aspect of linguistic structure," however, would be guaranteed to lead us astray.

First, here is an obvious methodological pitfall that we would be throwing ourselves into willingly. Not finding structural priming for a certain representation might have many different causes, the representation not existing being only one of them. It might also be that we haven't done the right experiment, or that the effect is too weak to detect. We might be unable to control for important confounding variables, be unable to find the proper stimulus materials, or lack the necessary statistical power. The key principle here is that absence of evidence is not evidence of absence.

Second, it is possible that the representation under investigation does, in fact, exist, but is for some reason not primeable in psycholinguistic experiments. There is no reason to expect that all linguistic representations posited in our theories are primeable. To be able to control for possible effects of meaning, most demonstrations of structural priming have involved a relatively small set of syntactic phenomena, such as the dative alternation or active vs. passive constructions (Mahowald et al. 2016b). Does this mean that other syntactic constructions (e.g., small clauses) do not exist? As an example from a different linguistic domain, Tooley et al. (2014) found that they could not prime intonational phrases. But that should not (and did not) lead the authors to conclude that intonational phrases lack representation.

More generally, the astounding progress that the cognitive sciences in general, and psycholinguistics specifically, have made since the cognitive revolution is founded upon the post-behaviorist assumption that it is legitimate to posit internal representations and processes even if not every one of these components can be proved by direct demonstrations of their existence. Edward Tolman and his colleagues (Tolman 1948) provided convincing evidence of the existence of map-like mental representations in rats, but this never involved priming them.

As for abandoning acceptability judgments, the authors' proposal amounts to sawing off the branch we're sitting on. The success story of structural priming owes a large debt to half a century of previously developed linguistic theory about syntactic structures, massively informed by acceptability judgments. Without linguists identifying grammatical phenomena, determining their scope, and studying the relationships between different syntactic forms, we wouldn't even know where to start looking. How else would we know that The pirate gave the princess a parcel and The pirate gave a parcel to the princess (or an active and a passive version of a sentence) are semantically equivalent if not through using native-speakers' judgments to establish that they are both acceptable for describing a certain state of affairs? In fact, it would be impossible even to develop experimental stimuli without using our (implicit) acceptability judgments; there is a reason that structural priming experiments usually don't contain sentences such as Pirate a parcel the princess the gave.

Structural priming certainly deserves its place in the vast array of methods available to psycholinguists. But we see no reason to give it primacy over the many other paradigms that have been, and still are, essential pillars of cognitive science.

\section{If priming is graded rather than all-or-none, can reactivating abstract structures be the underlying mechanism?}

doi:10.1017/S0140525X17000358, e287

Laurie Beth Feldman ${ }^{\mathrm{a}}$ and Petar Milin ${ }^{\mathrm{b}}$

${ }^{a}$ Department of Psychology, State University of New York at Albany, Albany,

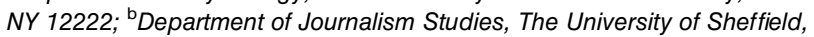
Sheffield S1 4DT, United Kingdom.

Ifeldman@albany.edu p.milin@sheffield.ac.uk

Abstract: In our commentary on Branigan \& Pickering (B\&P), we start by arguing that the authors implicitly adopt several assumptions, the consequence of which is to make further claims necessary and/or sufficient. Crucially, the authors assume the existence of discrete units at various levels of linguistic granularity that then must be operated upon by combinatorial mechanisms and rules (i.e., decomposition/ recomposition). They further argue that structural priming provides a powerful tool to study abstract, structural representations. We provide evidence that priming effects in production are characterized better as graded than as all-or-none and that priming need not arise from a mechanism that (re)activates a shared but abstract internal structure.

Branigan \& Pickering (B\&P) work within a framework that posits discrete linguistic units at various levels of granularity that must be operated upon by combinatorial mechanisms and rules (i.e., decomposition/recomposition). They argue that structural priming provides a powerful tool to study abstract, structural representations, but there are alternatives that better embrace the broader communicative function of language (Baayen et al. 2016a; 2016b). However, even within their framework, the experimental priming methodology that $\mathrm{B} \& \mathrm{P}$ depend on for their argument is more nuanced than what they have explored with choice between two syntactic structures as their measure of behavior. They assert that abstract structural processes can be studied independently from the contributions of individual words, because priming arises even when words do not reappear. Often, however, priming effects are not all-or-none, and effect sizes depend on what recurs. Thus, systematically graded priming outcomes challenge the descriptive adequacy of B\&P's theorizing about how lexical and syntactic knowledge interact. In contrast to $\mathrm{B} \& \mathrm{P}$, we assert that priming effects in production are more informative when characterized as graded than as all-or-none and that priming need not arise from a mechanism that (re)activates a shared but abstract internal structure. We present examples from our own work that show systematic variation among the "structures" that generate priming, eschew a division between representation and process, and exploit rather than tolerate differences among words.

In a single-word inflected production task, the verb stem constitutes the structure that recurs, and the requisite production - an inflected verb form - is specified by instruction rather than by a sentence context. Admittedly, this version of structural priming is severely constrained. Nonetheless, we have demonstrated that reaction time (RT) differences between regular ed and ing productions differ significantly more when primed by a written stem than by a drawing of the action depicted by the stem (Feldman et al. 2013a; 2013b). These prime modality (drawing, word) differences impact the magnitude of priming rather than its presence or absence. Relative differences such as these 
constrain the abstractness of lemma representations and impose limitations on the independence of structural priming from lexical contributions.

In addition, differences in production times between verbs with high and low lemma frequency are larger when generating progressive (ing) than past tense $(e d)$ inflected forms of regular verbs. These reliable differences (RT, accuracy) between various inflected word forms of the same verbs pose a challenge to an account based only on binding between a constituent structure rule and a lexical representation without reference to "features like tense, number or aspect."

Admittedly, we confine structural priming to inflected word forms rather than sentential syntax. We emphasize, however, that this is a useful trade-off, in that the task generates RT as well as accuracy data, and having both eliminates some of the challenges that typically arise with the dichotomous data generated by the classical structural-choice priming task. At a minimum, graded priming effects across variants of the structural priming methodology highlight the potential interdependence between lexical contributions and syntactic processing, and challenge the descriptive adequacy of the $\mathrm{B} \& \mathrm{P}$ account of structural priming.

When verbs recur in prime and target structures, there is a benefit to production termed a lexical boost (Cleland \& Pickering 2003). The existence of the lexical boost argues against a purely structural account of priming in which lexical information fails to make contact with the central syntactic component. Nonetheless, B\&P's structural priming account fails to anticipate graded, systematic lexical contributions due to differences among words.

In addition to manipulating degree of lexical specification (drawing, word) while matching output at production, we examined inflectional regularity. Lower accuracy for irregularly than for regularly inflected past-tense forms arose when generating a past-tense inflection from a verb stem but not from a drawing of the same action. Here, negative priming between input and output structures is possible when lexical information is specified orthographically but not by a drawing. Interactions of prime modality with regularity-such that a regularity effect manifests itself with productions from the written stem but not from a drawing of that same action - challenge the claim that the lexical boost in production derives simply from repetition of a particular lemma (e.g., dive) that is unspecified for shared features such as tense, number, or aspect (Pickering \& Branigan 1998). Productions that share a lemma and convey the same action but prime differently depending on the availability of the stem, set limits on the abstractness of the representations that purportedly produce structural priming.

Absent from the $\mathrm{B} \& \mathrm{P}$ account of structural priming, even when enhanced by lexical boost, is an appreciation of the communicative function of language and the requisite system's priority for reducing uncertainty and exploiting typicality (cf., Ramscar et al. 2013). Elsewhere, we have argued for the benefits of discrimination-based predictors in priming over more conventional lexical-distributional predictors (Milin et al. 2017). Key is that priming reflects not only the "similarities" between prime and target, but also the similarities of the prime and the target to other words. Surely an appreciation of systematic differences in the probability distributions of the various alternatives deserves consideration such that all structural matches are not equivalent. Similarly, anticipating variation with respect to particular lexical entries and the syntactic relations in which they potentially participate by introducing prime and target items as random effects in analyses enriches insights into any variant of priming (Milin et al. 2017).

While structural priming may provide a useful method of investigating linguistic knowledge with significant benefits over acceptability judgments, the nuances of stem-based (as distinguished from drawing-based) priming effects as well as a more functional characterization of syntactic patterning leads us to question whether the structural priming effect that $\mathrm{B} \& \mathrm{P}$ endorse is best characterized in terms of the presence or absence of (re)activation of purely abstract syntactic representations.

\section{Structural priming can inform syntactic analyses of partially grammaticalized constructions}

\section{doi:10.1017/S0140525X1700036X, e288}

\section{Elaine J. Francis \\ Department of English, Purdue University, West Lafayette, IN 47907-2038. \\ ejfranci@purdue.edu \\ http://web.ics.purdue.edu/ ejfranci/ejfrancis.htm}

Abstract: Branigan \& Pickering (B\&P) argue successfully that structural priming provides valuable information for developing psychologically plausible syntactic and semantic theories. I discuss how their approach can be used to help determine whether partially grammaticalized constructions that have undergone semantic change also have undergone syntactic reanalysis. I then consider cases in which evidence from priming cannot distinguish between competing syntactic analyses.

Branigan \& Pickering (B\&P) argue that evidence from structural priming informs our understanding of abstract linguistic representations in ways that traditional acceptability judgments do not, thus providing a valuable tool for developing psychologically plausible theories of syntax and semantics. I agree with them on this point and believe their approach can shed light on tricky cases of grammaticalization in progress, as suggested below. However, I maintain that evidence from structural priming can be ambiguous with respect to the influence of syntactic structure alone versus syntax-semantics mappings and, therefore, cannot distinguish by itself between competing syntactic analyses.

Examining data from dozens of published studies that have used structural priming to investigate language processing, $\mathrm{B} \& \mathrm{P}$ propose a theoretical approach similar to Culicover and Jackendoff's (2005) Simpler Syntax. According to B\&P's approach, syntax consists of a "shallow" constituent structure without any movement transformations and minimal null constituents; thematic roles, event structure, and quantificational information are included only in semantics. Supporting this view, they cite evidence showing that speakers are sensitive to shallow syntax even when the semantic argument structure differs between prime and target (Bock \& Loebell 1990; Flett 2006; Wittenberg 2014). I suggest that B\&P's approach can be fruitfully applied also to cases in which nouns, verbs, or adjectives have undergone partial grammaticalization. Such cases are notoriously challenging for synchronic theories of syntactic representation because the items in question show mixed properties of lexical and functional categories (Denison 2010). Francis and Yuasa (2008) argued, based on evidence from English, Japanese, and Cantonese, that at least some such cases involve semantic change in the absence of syntactic reanalysis - a phenomenon they captured synchronically using a parallel-architecture representation (Culicover \& Jackendoff 2005; Sadock 1991). For example, English quantificational nouns (e.g., lot, bunch, ton) display mixed properties of collective nouns (e.g., bundle), and quantifiers (e.g., many), with the quantifier-like properties due entirely to semantics. Thus, quantificational NPs (e.g., a lot of sticks) share a syntactic representation with collective NPs (e.g., a bundle of sticks), despite differences in meaning. In both types of NP, the first noun (lot, bundle) acts as the syntactic head of the phrase. This analysis relied on attributing some patterns of acceptability judgments to syntax and others, to semantics. Following B\&P's proposal, one could test whether collective NPs would prime the production of quantificational NPs when speakers are asked to describe a set of objects in terms of quantity. Such a priming effect would support Francis and Yuasa's proposal that quantificational nouns really do act as head nouns in syntax, while the absence of any priming effect would suggest that quantificational NPs and collective NPs differ syntactically. More generally, at least for cases in which the source construction continues to exist in the language alongside the grammaticalized form, priming tasks could help determine 
Commentary/Branigan and Pickering: An experimental approach to linguistic representation

whether a lexical item or construction that has undergone semantic change also has undergone syntactic reanalysis.

It is less clear, however, how to interpret situations in which priming effects appear in different degrees for different types of primes. B\&P cite studies showing an enhanced priming effect when the prime and target share not only the same shallow syntactic representation, but also the same abstract syntax-semantics mapping (Griffin \& Weinstein-Tull 2003; Raffray et al. 2014). Raffray et al. (2014) found that sentences with a coerced (missing) predicate (e.g., The celebrity began the champagne) primed target responses with a coerced predicate more effectively than did syntactically similar sentences with an event NP (e.g., The celebrity began the speech). However, these two types of NP-V$\mathrm{NP}$ sentences were alike in failing to prime NP-V-VP responses (e.g., The celebrity began drinking the champagne). B\&P take the latter fact to mean that the missing predicate of a coerced sentence is not represented in the syntax. But what, then, do we make of the fact that coerced predicate sentences primed coerced predicate responses more strongly than event NP sentences did? Raffray et al. (2014, p. 97) propose that speakers were sensitive to particular syntax-semantics mappings, in addition to being sensitive to shallow syntax. It seems, however, that one could plausibly interpret the different degrees of priming to signal some subtle difference in the syntactic representations.

Similarly, Griffin and Weinstein-Tull (2003) found that objectraising infinitives (e.g., John believed Mary to be nice) primed object-raising responses (as opposed to finite paraphrases) more effectively than object-control infinitives did (e.g., John persuaded Mary to be nice). Griffin and Weinstein-Tull (2003, p. 549) interpreted these findings to mean that language users are sensitive to the similarity of abstract syntax-semantics mappings between prime and target, because object-control infinitives have an additional argument role. As an alternative to this explanation, $\mathrm{B} \& \mathrm{P}$ suggest that perhaps the additional argument role in object-control sentences is associated with a distinct syntactic representation, meaning that reference to syntax-semantics mapping is not necessary to explain the results (note 6 in the target article). While Griffin and Weinstein-Tull's explanation is compatible with parallel-architecture theories such as Simpler Syntax (Culicover \& Jackendoff 2005) and Construction Grammar (Goldberg 2006) - which do not permit null constituents in the syntax-B\&P's alternative requires a null constituent in the syntax. Therefore, B\&P's explanation is more compatible with the standard generative account of object control, in which the infinitive subject is a null pronoun (PRO) (Chomsky 1981). As B\&P acknowledge, the evidence does not distinguish between these two possible syntactic representations.

Evidence from priming is useful for showing speakers' sensitivity to structural similarity. As such, it can indicate which elements must be included in syntactic representations (e.g., syntactic categories, constituent ordering) and whether historically related constructions continue to share a constituent structure. It cannot tell us, however, whether differences in structure, as reflected in differences in degrees of priming, are due to differences in syntactic representation alone or to syntax-semantics mappings. While structural priming provides valuable evidence for linguistic theory building, the abstract representational basis for any particular facilitation effect remains subject to interpretation. A psychologically plausible theory of syntactic and semantic representation, therefore, must take into account many different types of evidence, each of which has its own advantages and limitations.

\section{The logic of syntactic priming and acceptability judgments}

\author{
doi:10.1017/S0140525X17000371, e289
}

Phoebe Gaston, Nick Huang, and Colin Phillips
Department of Linguistics, University of Maryland, College Park, MD 20742.

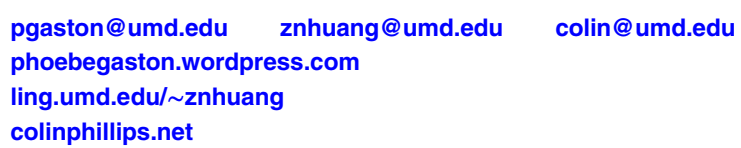

colinphillips.net

Abstract: A critical flaw in Branigan \& Pickering's (B\&P's) advocacy of structural priming is the absence of a theory of priming. This undermines their claims about the value of priming as a methodology. In contrast, acceptability judgments enable clearer inferences about structure. It is important to engage thoroughly with the logic behind different structural diagnostics.

Branigan \& Pickering $(\mathrm{B} \& \mathrm{P})$ wish that structural priming would get more love as a source of evidence about linguistic structures. We certainly welcome all sources of relevant evidence. Their case, however, would be helped if they engaged more thoroughly with the logic underlying priming and standard acceptability judgment (AJ) evidence. Their disparagement of linguistics is unhelpful, but we leave it to other commentators to address those issues.

Most importantly, B\&P provide no account of syntactic priming and the circumstances in which it should obtain, beyond the obvious fact that priming reflects similarity of some kind. This makes it difficult to draw clear conclusions from priming findings. Meanwhile, they miss key aspects of how acceptability judgments are used to draw inferences about linguistic structure.

$\mathrm{B} \& \mathrm{P}$ claim that $\mathrm{AJ}$ merely diagnoses set membership (i.e., whether a sentence is possible or impossible), whereas priming is more powerful because it additionally diagnoses similarity between strings. This is incorrect. AJ is routinely used to test paradigms of closely related sentences in order to diagnose specific representational properties (e.g., identity [coordination, ellipsis], structural hierarchy [binding], or locality [relativization, wh-movement]). These tools do not diagnose all properties that we might care about, but when they work, they allow precise inferences. In contrast, priming diagnoses similarity in a less precise fashion. A pair of sentences can be similar in multiple different ways, so the finding that they prime one another allows us to draw only weak inferences. A more explicit account of structural priming could sharpen B\&P's arguments. We suspect that they assume priming requires a form of structural identity in which the prime and target include a single piece of structure that is identical between the two (e.g., a verb phrase), and that structure has identical daughters (e.g., the verb phrase has a ternary branching internal structure). This is different from the weaker hypothesis that, for example, the prime and target must share a sequence of syntactic nodes, even if those nodes are not structured identically. The evidence for the stronger hypothesis is not provided, and it is not clear what such evidence would look like.

Priming is not well suited to identifying differences rather than similarities between structures. We contrast this with arguments that can be constructed using patterns of AJ. These sometimes provide evidence that superficially different constructions are structurally similar (e.g., comparative constructions and wh-questions are subject to the same locality restrictions [Bresnan 1975]). Sometimes, the converse is true: Constructions that are superficially similar are actually structurally different (e.g., control vs. exceptional case marking constructions).

To take a specific example from the paper: priming between unaccusative and unergative constructions in Spanish only provides evidence that the two constructions are similar at some level, as is evident from their surface syntax. Nobody disputes that they have something in common. The interesting contribution from various kinds of $\mathrm{AJ}$ evidence is that unaccusatives and unergatives are not structurally identical and have differences that are generally not obvious in surface forms (Levin \& Rappaport 1995).

Therefore, from our perspective, AJ is the more versatile and cost-effective tool, with a more varied set of specific diagnostics that can identify hierarchical relations, constituency relations, and varieties of long-distance relations, in contrast to priming's rather vague indication of structural similarity at some level. 
However, we acknowledge that there is a potential for priming and AJ to provide complementary evidence under a more developed theory of priming than is currently offered.

$\mathrm{B} \& \mathrm{P}$ are quick to dismiss results from standard AJ diagnostics of constituency, pointing out the well-known fact that different diagnostics do not always converge straightforwardly. This strikes us as an odd strategy. Reliably conflicting results should prompt one to re-examine rather than discard the data or the methodology. Constituency diagnostics do indeed produce apparent conflicts under standard assumptions; this is a gift to the researcher rather than a threat, however, because it invites us to dig deeper and to understand better how the diagnostics work. Such efforts have proven fruitful (e.g., Pesetsky 1995; Steedman 2000; Phillips 2003).

As far as we can tell, the priming literature, including almost all studies carried out by the authors, depends on properties that are independently diagnosed by AJ. For example, in many studies, the authors presuppose the existence of PPs, VPs, and NPs, all of which are constructs derived from AJ. If AJ data are as flawed as the authors suggest, then this is problematic for priming studies that take basic AJ findings as a starting point. It is unclear how constituency could be identified solely by priming.

Finally, B\&P argue that priming evidence supports a monostratal view of syntax (i.e., a single level of syntactic representation), in contrast to transformational accounts in which multiple representations are related to one another by movement operations. We acknowledge the importance of the issue, but it is unclear how the priming evidence bears on it. Everybody acknowledges that sentences encode different types of relations: thematic roles, grammatical relations (e.g., subject, direct object), scope, topic/ focus, etc. This is not in dispute. The disagreements surround the question of how these various relations are structurally encoded and how the structural encodings are related to one another. Transformational accounts are one hypothesis about the relation between the encodings, but all accounts must offer an account of the same problems. What kind of priming result, in principle, could falsify the authors' belief that syntax is monostratal and provide positive evidence for more than one level of representation? We know of no such evidence.

In sum, the authors should articulate a more explicit theory of priming, what it can and cannot diagnose, and how it relates to evidence from other tests. Priming evidence has the potential to complement AJ data, but priming evidence will be taken more seriously by those steeped in AJ (and other methods) if there is a genuine attempt to engage with the logic behind the various tests.

\section{ACKNOWLEDGMENT}

This material is based upon work supported by the National Science Foundation under Grant No. 1449815.

\section{Converging on a theory of language through multiple methods}

doi:10.1017/S0140525X17000383, e290

\author{
Mónica González-Márquez, ${ }^{a}$ Michele I. Feist, ${ }^{\mathrm{b}}$ and \\ Liane Ströbel ${ }^{a}$ \\ a Institute for English, American and Romance Studies, RWTH-Aachen \\ University, 52056 Aachen, Germany; ${ }^{b}$ Department of English, University of \\ Louisiana at Lafayette, Lafayette, LA 70504. \\ monica.gonzalez-marquez@ifaar.rwth-aachen.de \\ feist@louisiana.edu liane.stroebel@ifaar.rwth-aachen.de
}

Abstract: Assuming that linguistic representation has been studied only by
linguists using grammaticality judgments, Branigan \& Pickering (B\&P)
present structural priming as a novel alternative. We show that their
assumptions are incorrect for cognitive-functional linguistics, exposing
converging perspectives on form/meaning pairings between generativists
and cognitive-functional linguists that we hope will spark the cross- disciplinary discussion necessary to produce a cognitively plausible model of linguistic representation.

We agree with Branigan \& Pickering's (B\&P’s) general argument that, to understand how language works, we must take into account both representation and processing. However, we take issue with several of the specific arguments put forth by the authors. These are:

That acceptability judgments are the only tool available to linguists;

That structural priming is the only alternative to acceptability judgments;

That linguists and psychologists don't work together to investigate linguistic representation.

These arguments are true only if we assume that linguistic representation is as defined by generative grammar: the result of an encapsulated system in which syntactic structure is divorced from meaning, and meaning is construed in formalist terms (Heim \& Kratzer 1998; Schiffer 2015). Vis-à-vis the broader language research community, the generativist view is mischaracterized as enjoying global consensus when, in fact, it has been opposed rigorously for decades by other linguistic traditions - in particular, by researchers within the cognitive-functional tradition. The direct consequence of this myopia is imagining that the limitations built into the generativist paradigm, in fact, are limitations on the entire field.

It is a truism in science that how a question is asked determines the type of answers that can be sought. Cognitive functional linguistics diverges from the generative school by assuming that there is an intimate connection between the form of language and the meaning that it communicates, with the consequence that neither form nor meaning can be studied in isolation. With this assumption comes Lakoff's (1990) "Cognitive Commitment": to ensure that what is posited regarding linguistic structure and representation is in accord with findings from other disciplines regarding the mind and the brain, along with a recognition that the development of cognitively plausible theories of language will require dialog with scholars in neighboring disciplines (cf., Tomasello 1998; 2003b). These commitments establish from the outset that understanding linguistic representation must be a multidisciplinary undertaking. The fulfillment of these commitments can be found in the growing number of research articles and books investigating linguistic representation using dozens of methodologies either borrowed from, or developed in conjunction with, multiple neighboring disciplines. These commitments are so important that there is a long-standing workshop series, the Empirical Methods in Cognitive Linguistics Workshops, whose primary goal is to bring together researchers from a wide variety of fields who seek to strengthen their methodological repertoires for the study of language and cognition.

As a field, cognitive-functional linguistics has brought together the following methods, among others:

Analyses of corpus data (e.g., Divjak \& Arppe 2013; Yoon \& Gries 2016),

Behavioral experiments (e.g., Dabrowska 2014; Feist 2010; 2013; Liu \& Bergen 2016; Bergen \& Coulson 2006; Winter \& Matlock 2013), Eye-tracking studies (e.g., Huette et al. 2014),

Integration of acoustic and linguistic cues (e.g., Walker et al. 2013), Studies of the interface between language and perception (e.g., Winawer et al. 2008; Lupyan 2012),

Gesture research (e.g., Núñez \& Sweetser 2006),

Linguistic analyses (e.g., Haspelmath 2008),

Sensorimotor concepts (Ströbel 2016),

Statistical analyses of cross-linguistic variation (e.g., Croft \& Poole 2008; Feist 2008; Levinson \& Meira 2003),

Neuropsycholinguistic experiments (e.g., Van Petten et al. 1999; Coulson \& Van Petten 2002; Saj et al. 2014; Perry \& Lupyan 2014). 
This short list of methods used in the study of linguistic representation is representative of the massive collective efforts that have yielded a view of language in which:

1. Prototype structures and schemas are evident in representational categories at multiple linguistic levels, including semantic (e.g., Lakoff 1987; Spivey et al. 2004), morphological (Bybee \& Moder 1983), and phonological (Bybee 1994);

2. There is no clear separation between levels of linguistic representation (Fillmore et al. 1988; Goldberg 2003);

3. Meaning is based on usage and experience, resulting in the existence of highly interconnected conceptual networks (Fillmore 1976; Ströbel 2016);

4. Meaning is context-dependent and dynamically created online, fed by multiple sources of available information (Feist \& Duffy 2015; Lupyan \& Casasanto 2015).

There is a gentle irony to the conclusion B\&P reach at the end of their article. Their chosen method, structural priming, led them away from the classical generativist proposal to conclude that structure and meaning cannot be studied in isolation, making their views ultimately consistent with the cognitive-functional tradition. This convergence suggests a way forward: that we all come to the table with our disparate lines of research to create a cognitively plausible model of linguistic representation. A similar suggestion was made by Jackendoff (2002) when he attempted to create a cornerstone for the convergence of linguistic theory. Unfortunately, little came of his efforts, because of, in part, the sparseness of outreach to researchers in the cognitive-functional tradition (cf., Spivey \& Gonzalez-Marquez 2003). In reintroducing this issue, we hope to spark conversations that will help advance our understanding of the representation and processing of language.

In sum, we don't take issue with the view of language B\&P ultimately propose; in fact, we tend to agree with it. Our point is to underline that this view is not novel, and that in integrating these findings with other proposals and findings, we can broaden our understanding of the linguistic representations that underlie speakers' capabilities.

\section{A usage-based cognitive linguistic (re-) interpretation of priming evidence}

\section{doi:10.1017/S0140525X17000395, e291}

\section{Franziska Günther}

Department of English and American Studies, Ludwig-Maximilians-University Munich, 80799 Munich, Germany.

franziska.guenther@anglistik.uni-muenchen.de

www.anglistik.uni-muenchen.de/personen/wiss_ma/guenther/index.html

Abstract: Usage-based cognitive linguistic (UBCL) theories offer a unifying interpretation of the different (structural vs. [more] lexical) priming effects reported by Branigan \& Pickering $(\mathrm{B} \& \mathrm{P})$, and they provide an ideal basis for explaining contextual influences on priming. However, they also call into question B\&P's claim that priming "provides evidence that is directly informative about mental representation" (sect. 1.5, para. 1).

I fully agree with Branigan \& Pickering $(\mathrm{B} \& \mathrm{P})$ that it is high time psychologists, psycholinguists, and linguists join forces in investigating linguistic knowledge. I also agree that priming experiments can contribute to arriving at a better, cognitively more realistic understanding of language. Priming evidence, however, is not immune to theoretical bias. I will illustrate this by approaching the priming effects reported by $\mathrm{B} \& \mathrm{P}$ from the perspective of usage-based cognitive linguistics (UBCL) (e.g., Croft 2001; Goldberg 1995, 2003, 2006; Langacker 2000, 2008; The Five Graces
Group 2009; Schmid 2015); that is, I will (re-)interpret them based on the following assumptions:

"Language is an integral part of human cognition" (Langacker 1987, p. 12);

Linguistic knowledge is highly dynamic and context-adaptive; it emerges from situated usage and is therefore potentially changed by every single usage experience;

Rules emerge from generalization processes; they take the form of schematic form-meaning pairs (constructions); that is, they are equivalent in nature and structure to specific lexical items or multi-word units. Consequently, grammar/syntax and lexicon form a continuum;

Knowledge of language is organized in a complex network of constructions. This network:

- Licenses redundancy, that is, contains both specific linguistic structures and their different degrees of abstractions, as well as both larger (e.g., sentence-level) and smaller constructions (e.g., single lexical items);

- Is characterized by multiple (types of) relations that the single constructions (as well as their form- or meaning-sides only) can hold to each other.

These UBCL principles suggest alternative interpretations of priming effects to those proposed by $\mathrm{B} \& \mathrm{P}$.

Most centrally, they allow for structural priming to be interpreted differently than as evidence in support of the autonomy of syntax. In UBCL terms, the only relevant difference between structural and (more) lexical priming effects is that schematic rather than specific form-meaning pairs are primed. This interpretation allows for all instances of priming (including lexical priming) to be modeled in a unitary fashion: as based on the same mechanisms and as applying to different levels of the same network of linguistic knowledge. Because this network also contains semi-specific elements (e.g., [NP go PP]; see, e.g., Goldberg 2003), the UBCL view of priming also readily accommodates lexical boost effects. Thus, from a UBCL perspective, the major potential of using priming to investigate linguistic knowledge does not so much lie in differentiating between syntactic and lexical processes and representations, but in identifying which level(s) of the constructional network language users actually and/or habitually access (see also Tomasello 2006, p. 3).

This construction-based UBCL view also suggests that structural priming effects (just as lexical priming effects; see $B \& P$, sect. 1.4, para. 3) might not occur only when "prime and target involve the same construction" (B\&P, note 10$)$, but also when the constructions involved in prime and target processing are (closely) related. The relations driving priming effects can be of different types (e.g., schema-instance, part-whole, similarity, contrast, etc.) and can variably hold between entire constructions or between their form- or meaning-sides only (e.g., Langacker 1987, p. 13). Findings of priming between idioms and formally parallel non-idiomatic utterances (Konopka \& Bock 2009), therefore, do not contradict construction-based UBCL models of language (as claimed by B\&P, note 10). The same accounts for priming between sentences in which different thematic roles and/or syntactic functions are formally realized in the same way (e.g., B\&P, sect. 2.1, para. 2; Pickering \& Ferreira 2008). Instead, from a UBCL perspective, such findings are potentially informative of the internal relational structure of linguistic knowledge and the way it is used in processing.

Priming experiments thus also prove a valuable tool for language research when approached from a UBCL perspective. However, some UBCL assumptions call into question what $\mathrm{B} \& \mathrm{P}$ define as one main benefit of structural priming - namely, that it "provides evidence that is directly informative about mental representation" (sect. 1.5, para. 1).

One of those assumptions is that information is represented redundantly in the language network. It suggests that very different kinds and combinations of constructions can be involved in the processing of what is-formally-the same utterance (e.g., 
Günther 2016, p. 143; Stefanowitsch \& Flach 2016, pp. 105-107); that is, an utterance such as The bottle is on the table might variably be accessed as a single chunk or involve the integration of several more schematic and more specific constructions (e.g., $[N P$ is on $N P]+[$ the bottle $]+[$ the table $]$, etc.). It is thus hardly possible to draw reliable conclusions about which and how many constructions a speaker used when producing or comprehending a particular utterance in a priming experiment.

What complicates matters even further is that, on a usage-based view (e.g., Günther 2016), the sets of constructions language users access during utterance production or comprehension are highly likely to differ among individuals and may vary even within individuals across external (situational, social, and linguistic) contextual conditions, as well as across time. Interindividual and crosscontextual variation mainly constrain the generalizability of observations from priming, and therefore call for a more systematic investigation of individual- and context-specific effects. Variation across time-which derives from the UBCL principle that every single episode of language use can have a permanent impact on linguistic knowledge-proves more problematic: It entails that, by testing it, priming experiments are changing the very representational system they wish to investigate. In usagebased understanding, such training effects are far from trivial: They affect the very essence of a language system that consistently reconstitutes itself through change and adaptation (e.g., Schmid 2015).

In sum, UBCL-based interpretations of priming effects prove to be very different from those made by B\&P based on different theoretical (pre-)assumptions (e.g., that syntax and semantics are separate, sect. 2 , para. 3 . They thus also yield a very different picture of what can be the function of priming in language research. This clearly illustrates that priming experiments cannot "discriminat $[\mathrm{e}]$ among ... accounts" of linguistic representations (B\&P, sect. 1.2, para. 10) - or at least cannot do so if used as a single method and if conducted and interpreted with specific preconceptions about the nature of linguistic knowledge in mind.

\section{Don't forget the neurobiology: An experimental approach to linguistic representation}

doi:10.1017/S0140525X17000401, e292

\section{Peter Hagoort \\ Donders Institute for Brain, Cognition and Behaviour, Max Planck Institute for Psycholinguistics, 6500 HB Nijmegen, Netherlands. \\ peter.hagoort@donders.ru.nl \\ www.mpi.nl}

Abstract: Acceptability judgments are no longer acceptable as the holy grail for testing the nature of linguistic representations. Experimental and quantitative methods should be used to test theoretical claims in psycholinguistics. These methods should include not only behavior, but also the more recent possibilities to probe the neural codes for language-relevant representations.

I am in full agreement with Branigan \& Pickering (B\&P) that an experimental approach to linguistic representation is necessary. Despite the central role of grammaticality judgment in the field of linguistics, it does not suffice. The reason is that, in many cases, this procedure does not obey the same quantitative standards (including the proper statistics) that are state of the art in the rest of cognitive science (cf. Gibson \& Fedorenko 2010). When I made this argument in a panel discussion at a major linguistics conference, the following counterargument was presented in a commentary:

When linguists evaluate contrasts between two (or more) sentence types, they normally run several different examples in their heads, they look for potential confounds, and consult other colleagues (and sometimes naive participants), who evaluate the sentence types in the same fashion. The fact that this whole set of procedures (aka, experiments) is conducted informally does not mean it is not conducted carefully and systematically. (Almeida 2010, in Talking Brains blog debate, June 14, 2010)

Running sentences in your head and consulting a colleague is fine for discovering interesting phenomena and possible explanations (for the context of discovery, anything goes), but it does not suffice as the context of justification. We all are subject to confirmation bias. The fallibility of introspection is equally well known; it is a method that hence has fallen out of grace in psychology a long time ago. Therefore, to justify one's theory, empirical data have to be acquired and analyzed according to the quantitative standards of the other fields of cognitive science. In many circumstances, claims by an expert linguist of the form Sentence A is grammatical and sentence $B$ is ungrammatical will not suffice as a valid empirical data point in support of a specific linguistic theory. That is why I endorse an experimental and quantitative approach to investigations of linguistic representations.

Moreover, I concur with $\mathrm{B} \& \mathrm{P}$ that the experimental results of the many sentence priming studies that they reviewed are in agreement with the Parallel Architecture that Jackendoff (2002; in press) has proposed and for which the Memory Unification and Control (MUC) model is a neurobiological specification (Hagoort 2005; 2014; Hagoort \& Indefrey 2014). The MUC model specifies non-overlapping neuronal network contributions for the unification of semantic, syntactic, and phonological information, in accordance with the separate generative capacities for semantics, syntax, and phonology that Jackendoff proposes (2002; in press; Culicover \& Jackendoff 2005).

I believe that, next to the behavioral methods that B\&P advocate, it might be helpful to take experimental methods from neurobiology on board as well (cf., de Groot \& Hagoort, in press). For instance, a direct correlate of priming in behavior is fMRI adaptation. This is the phenomenon that the blood-oxygen-dependent (BOLD) response in neuronal populations sensitive to a stimulus attribute is suppressed or enhanced when that attribute is repeated (Grill-Spector et al. 2006). Using such an fMRI adaptation paradigm during speech comprehension and production, we found a clear segregation for areas involved in lexical processing, syntax, and semantics. However, for each of these domains, the same areas were recruited in speaking and listening (Menenti et al. 2011; Segaert et al. 2012). This supports the claim that lexical, syntactic, and semantic representations are segregated but shared for production and comprehension. In general, repetition suppression of the BOLD signal can provide insight into the representational features that a particular population of neurons codes for. In recent years, the method of Representational Similarity Analysis has been developed (Kriegeskort et al. 2008) to determine how representational information might be encoded in distributed patterns of brain activity.

The toolkit of cognitive neuroscience has expanded in the last few decades (cf., De Groot \& Hagoort, in press). This does not take away the need for linguistic theory and theory-driven questions. However, the context of justification has to meet the standards of the other branches of cognitive science. Clearly, acceptability judgments can no longer be seen as the holy grail for testing the nature of linguistic representations. There is no excuse for leaving the available experimental and quantitative methods unused in this domain of research.

\section{Acceptability judgments still matter: Deafness and documentation}

doi:10.1017/S0140525X17000413, e293

Matthew L. Hall, ${ }^{a}$ Rachel I. Mayberry, ${ }^{b}$ and Victor S. Ferreira ${ }^{c}$ ${ }^{a}$ Department of Linguistics, University of Connecticut, Storrs, CT 06269-1145; ${ }^{b}$ Linguistics Department, University of California at San Diego, La Jolla, CA 92037; ' Department of Psychology, University of California at San Diego, La Jolla, CA 92037. 
matthall.research@gmail.com

http://matthallresearch.com

rmayberry@ucsd.edu

http://mayberrylab.ucsd.edu/

vferreira@ucsd.edu

http://lpl.ucsd.edu/

Abstract: The target article's call to end reliance on acceptability judgments is premature. First, it restricts syntactic inquiry to cases were a semantically equivalent alternative is available. Second, priming studies require groups of participants who are linguistically homogenous and whose grammar is known to the researcher. These requirements would eliminate two major research areas: syntactic competence in d/Deaf individuals, and language documentation. (We follow the convention of using deaf to describe hearing levels, Deaf to describe cultural identity, and $d /$ Deaf to include both. Our own work has focused on Deaf signers, but the same concerns could apply to other deaf populations.)

In their abstract, Branigan \& Pickering (B\&P) assert that the advent of syntactic priming should "end the current reliance on acceptability judgments." We argue that this claim is too strong; although syntactic priming is useful for some research questions, acceptability judgments are still needed for other important questions.

B\&P anticipate one of our two major objections: that studying syntactic priming in production requires an alternative syntactic structure that expresses a semantically equivalent idea. Setting aside reasonable objections from construction grammarians, who may question whether different structures could ever be semantic equivalents (e.g. Goldberg 1995), such a restriction dramatically narrows the scope of syntactic inquiry, both within and across languages. B\&P's proposed solution is to measure syntactic priming in comprehension, where an alternative structure is not necessary. They also acknowledge, however, that the evidence for syntactic priming in comprehension has been much more scarce and fragile; this appears to contrast with their belief that syntactic priming research has reached maturation. Even if there were robust and reliable methods of measuring syntactic priming in comprehension, that would not reach the goal of achieving a unified theory of syntactic representation, in the same way that current research on language production mechanisms has not been particularly informative about theories of language comprehension.

Our second major objection is that syntactic priming studies require (1) that groups of participants be linguistically homogenous and (2) that their hypothesized grammar already be known to the researcher. These requirements would eliminate two critical areas of inquiry: (1) the study of the mental grammars of deaf individuals who were not exposed to language until after early childhood, and (2) traditional linguistic fieldwork.

Deaf individuals with delayed access to language cannot be assumed to share the internal grammar of native users or even of other late learners. Indeed, the nature of their mental grammar is often the very question under investigation. Furthermore, it is uncommon for these individuals to be available to participate in the numbers that are typically necessary to detect priming effects. What these questions require, then, is not a method that averages across many participants, but one that allows for the description of syntactic performance within and between individuals. Acceptability judgments allow this; syntactic priming does not.

Previous work, much of it accomplished using acceptability judgments, has shown that deaf individuals who are first exposed to an accessible language after early childhood exhibit variation in both phonology and morphosyntax compared with individuals immersed in language from birth, particularly with respect to more complex syntactic structures (Boudreault \& Mayberry 2006; Mayberry et al. 2002). Low syntactic performance, revealed by acceptability judgments, led us to ask whether Deaf non-native signers of American Sign Language (i.e., those first exposed to ASL after early childhood) would exhibit patterns of syntactic priming that varied from those of Deaf native ASL signers, or of hearing individuals who learned ASL as an L2 in adulthood (Hall et al. 2015). Contrary to our expectation, Deaf adults who had learned ASL after early childhood showed patterns of syntactic priming that were identical to those of Deaf native signers and hearing L2 signers.

If syntactic priming were our only tool, we might conclude that late exposure to a first language does not perturb syntactic representation. We view such a claim as premature, given substantial evidence that late learners struggle with syntax (especially with complex syntax) if they were not exposed to any linguistic system in childhood. For example, an fMRI study revealed substantial differences in the neural organization of ASL sentence processing in such individuals using acceptability judgments (Mayberry et al. 2011). Although we did not administer an acceptability judgment task to the participants in our ASL syntactic priming study, the Deaf late learners did show unusual patterns in phonological similarity judgments (Hall et al. 2012), suggesting that the results of the priming study may have failed to detect differences in syntactic processing.

One possible explanation relates to our first objection above: the type of structures that we can study with a priming paradigm is limited. ASL grammar is only partially described and syntactic processing differences among Deaf, atypical, learners are most pronounced on more complex structures, such as object relative clauses (Boudreault \& Mayberry 2006; Mayberry \& Lock 2003). However, ASL does not have an alternative structure for expressing object relative clauses; therefore, we were restricted to studying a simpler structure (prenominal vs. postnominal adjectives; $\mathrm{cf}$., Cleland \& Pickering 2003), where prior research has not detected differences between early and later learners (Boudreault \& Mayberry 2006; Mayberry \& Lock 2003).

One way to investigate syntactic acquisition in this population is the case study approach using well-established methods to study language acquisition longitudinally in a few individuals (Ferjan Ramirez et al. 2013). Syntactic priming is simply not a viable methodology for single-subject or small-group designs. This limitation applies to any case in which there is concern that an individual's grammar deviates from that of the broader linguistic community.

The second research area that would be jeopardized by exclusive reliance on syntactic priming is field linguistics. In many situations of language documentation, it is common for very few informants to be available (let alone cooperative). Gathering acceptability judgments from a small number of speakers enables documentation of the range of structures used by the world's languages, which, in turn, is essential to our understanding of linguistic representation. Syntactic priming is an insufficiently powerful tool to investigate these theoretically important situations.

Acceptability judgments can reveal what an individual implicitly knows about syntax in a way that syntactic priming cannot. In our view, both methods have their place, and more progress will be made by judiciously deploying each tool in its proper context.

\section{Syntactic levels, lexicalism, and ellipsis: The jury is still out}

\author{
doi:10.1017/S0140525X17000425, e294
}

\section{Robert J. Hartsuiker ${ }^{\mathrm{a}}$ and Sarah Bernolet ${ }^{\mathrm{b}}$}

${ }^{a}$ Department of Experimental Psychology, Ghent University, B-9000 Ghent, Belgium; ' Department of Linguistics, University of Antwerp, B-2000 Antwerp, Belgium.

robert.hartsuiker@ugent.be sarah.bernolet@uantwerpen.be

http://users.ugent.be/ rhartsui/

Abstract: Structural priming data are sometimes compatible with several theoretical views, as shown here for three key theoretical claims. One reason is that prime sentences affect multiple representational levels driving syntactic choice. Additionally, priming is affected by further 
cognitive functions (e.g., memory). We therefore see priming as a useful tool for the investigation of linguistic representation but not the only tool.

We are very sympathetic to Branigan \& Pickering's (B\&P's) general idea of proposing structural priming as a central part of a research programme aimed at understanding the nature of linguistic representation. Our concern with this programme, however, is that it overlooks important limitations of structural priming research. Such limitations concern the multiple levels at which priming can take place and the fact that priming experiments do not only recruit linguistic representations, but also other aspects of cognition such as memory and attention. Some of the key inferences B\&P make from priming results, therefore, can be accounted for differently. We argue that this is the case for the claims that (1) there is only a single syntactic level; (2) the lexical boost supports a lexicalist architecture; and (3) priming can straightforwardly detect whether there are syntactic representations for elided constituents.

$\mathrm{B} \& \mathrm{P}$ claim that there is only one level of syntactic representation, in contrast to the two-level view taken in some parts of theoretical linguistics (Pollard \& Sag 1994), speech error research (Garrett 1975), and computational modeling (Kempen \& Hoenkamp 1987). The main argument for the one-level view is Pickering et al.'s (2002) finding that "shifted" datives in English (1) do not prime the production of Prepositional Object (PO) datives (2) relative to a baseline (also see Pappert \& Pechmann 2014). $\mathrm{B} \& \mathrm{P}$ argue that, if there were an intermediate syntactic level that specifies syntactic relations but not order, the shifted and PO structures would share a representation at that level. The two-level view, therefore, would predict priming from shifted to PO structures (albeit weaker than priming from PO to PO, as both POs, of course, would share representations at both levels). However, this line of reasoning ignores the possibility of priming at the level of thematic roles (in terms of order or emphasis), a possibility B\&P do propose in several places. After all, a shifted dative has the same ordering of thematic roles as a Double Object (3), and indeed, priming at that level is supported by Bernolet et al. (2009), Chang et al. (2003), and Vernice et al. (2012). Thus, the data pattern is entirely compatible with an account in which a shifted sentence primes the PO because of overlap in non-linearized structure but also the DO, because of similarity in the ordering of thematic roles - resulting in comparable proportions of DO and PO responses in the baseline and after shifted datives.

(1) The racing driver showed to the helpful mechanic the problem with the car

(2) The patient showed his leg to the doctor

(3) The patient showed the doctor his leg

Another central claim is that the lexical boost to priming is directly reflective of the relation between the lexicon and syntax. However, alternative conceptualizations of this relation account for the lexical boost differently (e.g., Chang et al. 2006). Such accounts acknowledge that the results of structural priming experiments, as with any psychology experiment, are task-dependent, and therefore a function of participants' strategies, attentional foci, and memory. Specifically, participants in structural priming experiments may use explicit memory of previous sentences to help find a structure for the current sentence, and repeated lexical items may be particularly strong retrieval cues for this (Chang et al. 2006; Hartsuiker et al. 2008). Consistent with this account, Hartsuiker et al. (2008) demonstrated that the lexical boost is much shorter-lived than the priming effect itself, compatible with the idea that multiple fillers separating the prime and target sentences reduce the effectiveness of a repeated item as a retrieval cue (see Bernolet et al. 2016 for further discussion). In summary, although it may be tempting to make direct inferences about the representational level from priming patterns, such inferences may overlook the role of memory (and perhaps other aspects of the person, task, context).
A final claim is that structural priming is informative about the syntactic representation of constituents that are represented semantically but not phonologically, as in ellipsis and coercion. In several examples, however, the interpretation rests on tacit assumptions. Take Cai et al.'s (2013) example: The waitress would like to lend the sailor the gun. Being afraid of getting into trouble, the chef would not like to [lend the sailor the gun]. There was no DO priming for sentences with ellipsis, but there was DO priming for sentences containing the full constituent. The logic here depends on the tacit assumption that, in the sentences with ellipses, any syntactic representation of the elided constituent must be parallel to the initial version of that constituent. If this assumption is not correct, however, and participants have a tacit syntactic representation for lend the gun to the sailor in a reasonable number of cases, little DO priming can be expected. Additionally, in Raffray et al.'s (2014) study on coercion, no difference in priming of coerced sentences was found for the coerced sentence The celebrity began the champagne compared to the control sentence The celebrity began the speech, suggesting that there is no syntactic representation for a missing predicate (drinking) in the coerced sentence. This conclusion again depends on a tacit assumption, namely that, in the control sentence, the status of speech as an event prevents participants from postulating a predicate. But if participants would postulate a predicate (they might represent the celebrity beginning to read, say, or practice the speech), the control and coerced sentence should behave alike

Although we fully agree that structural priming is a promising tool for investigating syntactic representation, we doubt whether it should be the only tool. As we have argued here, priming patterns are often compatible with several accounts, and complicating factors are that (1) priming likely affects several levels of representation; (2) priming is not only a function of processes within the system of linguistic representations, but also of processes outside of it such as memory; and (3) the interpretation of priming experiments sometimes hinges on a priori assumptions.

\section{Structural priming supports grammatical networks}

doi:10.1017/S0140525X17000437, e295

\section{Richard Hudson}

Linguistic Department, University College London, London WC1E 6BT. r.hudson@ucl.ac.uk

www.dickhudson.com

Abstract: As Branigan \& Pickering (B\&P) argue, structural priming has important implications for the theory of language structure, but these implications go beyond those suggested. Priming implies a network structure, so the grammar must be a network and so must sentence structure. Instead of phrase structure, the most promising model for syntactic structure is enriched dependency structure, as in Word Grammar.

Branigan \& Pickering $(\mathrm{B} \& \mathrm{P})$ rightly argue that we theoretical linguists should pay attention to the massive evidence for structural priming which they review, but their argument actually suggests an even more radical direction for linguistic theory. In a nutshell, structural priming shows that grammars are networks; and if that's true, then linguists should be developing network-based models of grammar and of sentence structure.

Take Bock's classic experiment with which B\&P open their case. One passive sentence primes another - e.g., an experimental subject is more likely to produce a passive sentence describing lightning hitting a church tower after reading The referee was punched by one of the fans than in a neutral control situation. How does this influence work? For B\&P, "the neural 
Commentary/Branigan and Pickering: An experimental approach to linguistic representation

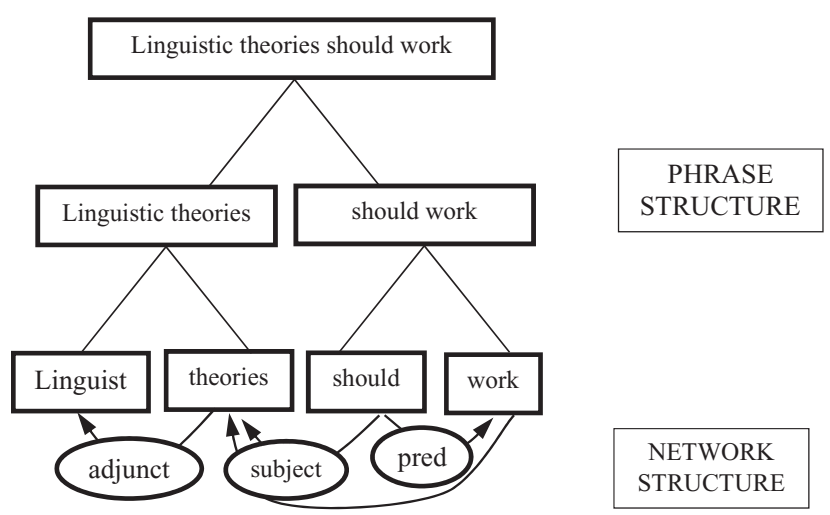

Figure 1 (Hudson). Phrase structure compared with network structure.

underpinnings of priming are not well understood," but the standard explanation for priming (Reisberg 2007, pp. 257-80) sees it as the effect of activation in a neural network spilling over from the intended target to network neighbours, thereby making the latter more accessible. In lexical priming, for example, reading nurse primes this word's network neighbours so that doctor becomes easier to retrieve than it would be otherwise. This explanation, however, makes sense only if knowledge is stored as a network of interconnected nodes; so the relevant units must be connected in a network, and if the units concerned are grammatical categories such as active and passive, these, too, must be part of a network.

This argument is familiar from the literature on connectionist models of processing and learning (Dell et al. 1999; Elman et al. 1996), but linguistic theories are pitched at a higher level of abstraction than the neurons that carry activation, so the two streams of research have hardly met. For B\&P, as for most linguists, language consists of abstract units such as words, phrases, categories, and relations; so, if these are part of a network, this must be a symbolic network. On the other hand, the activation responsible for priming in this network is a property of neural networks, so it is reasonable to assume that language is a symbolic network supported by a neural network. In other words, language belongs to the mind, while activation belongs to the brain.

The network view of language is widely accepted in modern theories of the lexicon (Allan 2006), with its multiple types of relation (meaning, realization, spelling, word class, and so on) and its many-to-many mappings. Structural priming shows that networks are just as relevant to syntax: A sentence's structure combines a network of patterns such as voice, tense, transitivity, and so on, each of which is sufficiently active to prime other examples of the same pattern. These patterns are the constraints of any constraint-based theory of syntax, including B\&P's preferred linguistic model, Parallel Architecture. In short, a sentence's grammatical structure must be a rich network of interacting and active nodes.

Where does this leave phrase structure, however, which is taken for granted in virtually every modern theory of syntax (and, disappointingly, by B\&P themselves)? Phrase structure is an extremely impoverished theory of the human mind that recognises only one possible mental relation: the part-whole relation between smaller and larger units. According to phrase structure, direct relations between individual words are not possible. For example, in the sentence Linguistic theories should work, the only possible relations are those shown in a tree such as the one above the words in Figure 1. For example, the word linguistic can be related to the phrase linguistic theories, but not to theories. Moreover, if phrase structure is right, phrases cannot intersect; so, if linguistic theories is part of the phrase linguistic theories should work, it cannot also be part of linguistic theories work. As we all know, however, both of these assumptions are really problematic:
Words do relate directly to one another (e.g., for agreement and government), and complex relations such as raising (from work to should) do exist.

Suppose, however, that syntactic theory is actually a network, not a tree. In that case, words can relate directly to one another, and multiple links are also possible. One such analysis is shown by the labeled arrows below the words in the figure for Linguistic theories should work. The labelled dependencies from theories to linguistic and from should to theories are typical of the very ancient tradition of dependency analysis (Percival 1990) and of more recent work in theoretical and descriptive linguistics (Tesnière 1959; 2015; Sgall et al. 1986; Mel’čuk 2009) as well as computational linguistics (Kübler et al. 2009) and psycholinguistics (Futrell et al. 2015; Gildea \& Temperley 2010; Jiang \& Liu 2015; Ninio 2006). All this work builds on the simple idea that our minds are free to recognise relations between words - an idea espoused some time ago by one of B\&P (Pickering \& Barry 1991).

The network notion, however, takes us further than this, to the idea that such relations need not be formally equivalent to a tree. In the example, theories is the subject not only of should, but also of work - a pattern that goes well beyond the formal limits of trees. This example illustrates the enriched dependency structure of one particular modern theory of grammar, Word Grammar (Duran-Eppler 2011; Gisborne 2010; Hudson 2007; 2010). In this theory, syntactic structure is so rich that it can even recognise mutual dependency in cases such as Who came?, in which who depends (as subject) on came and came depends (as complement) on who. Mutual dependency is absolutely impossible in any treebased theory, but of course, it is commonplace in ordinary cognition (e.g., in social structures).

In conclusion, structural priming shows not only that a grammar is a network, but also that enriched dependency structure is more plausible than phrase structure as a model of mental syntax.

\section{Action sequences instead of representational levels}

doi:10.1017/S0140525X17000449, e296

\section{Ruth Kempson ${ }^{\mathrm{a}}$ and Eleni Gregoromichelaki ${ }^{\mathrm{a}, \mathrm{b}}$}

aPhilosophy Department, King's College, London WC2R 2LS, United

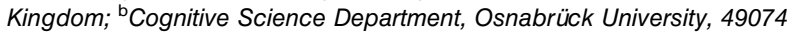
Osnabrück, Germany.

ruth.kempson@kcl.ac.uk

http://www.kcl.ac.uk/artshums/depts/philosophy/people/staff/associates/ emeritus/kempson/index.aspx

elenigregor@gmail.com

https://scholar.google.co.uk/citations?user=WnwSV4cAAAAJ\&hl=en

Abstract: Despite enthusiastic agreement that experimental data are directly relevant for determining grammar architecture, we present one main objection to the conclusions that the authors draw from their results: The data are perfectly compatible - in fact, much more in linewith an alternative that does not rely on syntactic representations. Instead, it is processing actions whose activation for comprehension/ production explains intra-/inter-speaker priming.

The target article is part of a welcome recent trend to take psycholinguistic results as able to adjudicate among competing theoretical proposals, rather than being treated as simply presupposing linguists' constructs. We wholeheartedly agree with this stance; in fact, we endorse it to a much greater extent than the authors advocate: From our point of view, the paper presents a rather conservative interpretation of the cited results in that it persists with the preoccupation of abstracting over behavioural/neuronal data to underlying abstract knowledge representations presumed to underlie their explanatory mechanisms (Gregoromichelaki \& Kempson, forthcoming; cf. Ferreira et al. 2008). 
We support the claim that methods of structural priming comparisons can be informative about mechanisms underlying linguistic processing. However, the authors argue that structural priming results are explainable only by assuming separate linguistic representations encoding semantic/syntactic/phonological information. Here, perhaps surprisingly, the authors seem to adopt the standard linguistic stance that theoretical frameworks/explanations need to presuppose an abstract, static view of linguistic knowledge, separating models of competence from accounts of performance.

In contrast, we propose an alternative formal architecture based on Dynamic Syntax (DS) as the syntactic engine (Cann et al. 2005; Kempson et al. 2001; 2017) enhanced with incremental construction of Type-Theory-with-Records (TTR; Cooper 2012) conceptual representations (DS-TTR; Gregoromichelaki et al. 2013; Hough 2015; Kempson et al. 2016; Purver et al. 2010). While eschewing a level of syntactic representation and any competence/performance distinction, such a framework is able to account directly for the priming data as well as standard linguistic generalisations.

Concentrating on syntax, the main focus of the paper, the data presented provide no evidence for theoretical or implementational perspectives on syntactic knowledge that would necessarily assume string-level hierarchical representations or accessing of stored well-formedness constraints in some kind of context-freegrammar format. Instead, a formal grammar adopting a DSTTR-style architecture consisting of routinised sequences of processing actions with no syntactic representations is much more compatible with the overall data. From this perspective, the appearance of abstract structural pattern-matching is epiphenomenal on the incrementality/predictivity of the processing of time-linearly unfolding stimuli. In contrast, the methodology that involves abstracting a level of syntactic representation over the actions impedes straightforward analyses of patterns of interlocutor coordination in dialogue (Gregoromichelaki et al. 2011).

Instead of syntactic hierarchical structure, according to DSTTR, a small set of elementary domain-general processing actions underpins both parsing and generation: Cross-linguistically available sequences of such actions cluster into higherorder sequential patterns (macros) that can be learned online, activated long-/short-term, and stored as chunks triggered by specific word forms (Eshghi et al. 2013). It is the reuse, and potential for adjustment, of such sequences that accounts for the authors' findings of "syntactic-pattern" repetitions appearing as distinct and/or independent from semantic interpretations. These results can be explained more explicitly in DS-TTR because the framework addresses the pervasive local ambiguity problem of incremental parsing/generation by predictive activation of various potential probabilistically weighted processing paths (sequences of actions). These processing paths are taken to constitute part of the context and some of them lead to identical TTR conceptual structures (Hough 2015; Hough \& Purver 2017; Sato 2011). For example, PO/DO or active/passive alternations in DS-TTR reflect the invocation of distinct sequences of actions to construct or linearise equivalent conceptual event frames (with distinguishing information-structure aspects reflected in the processing order). The parser/generator initially pursues the highest-ranked option, with the rest maintained in the context for conversational-repair purposes (Eshghi et al. 2015; Hough 2015). Success of one such path in achieving the intended conceptualisation will enhance the probability of perception/execution of the same action sequence subsequently if the word forms accessed make it available, while inhibiting the pursuance of alternatives.

Cumulative priming effects are predicted with additional repetition of the triggering word forms (the lexical boost effect), because phonological forms are stored in context for conversational purposes like clarification. Facilitation of retrieval is predicted even when repetition of the same word forms in conjunction with the same word order leads to distinct conceptual frames (Bock \& Loebell 1990), a mechanism independently needed for ellipsis resolution, or in priming across languages (given that code-switching in DS-TTR does not involve shift of processing environment [Gregoromichelaki 2017]).

The TTR conceptual frames invoked in processing explain the observation that speakers may show behaviour indicating that they represent semantic elements they do not hear/utter. However, with sequences of actions modelling incremental conceptual integration of stimuli, there is no need for postulating movement or empty categories while it is also predicted that long-distance dependencies of the standard kind should trigger parallel sequential patterns subsequently even in the absence of semantic parallelism. Finally, given that the DS-TTR modelling of the grammar itself is driven by the generation of predictions of upcoming sequences of actions, any already pursued action paths will always be prioritised (Myslín \& Levy 2016), tuning processing accordingly and thus explaining why comprehension is cross-primed by production and vice versa within and between speakers.

From this perspective, syntactic knowledge is not autonomous but derivative upon other forms of procedural knowledge, namely sequential action planning and comprehension with gradual elaboration of conceptual representations expressing stimuli categorisation as it occurs across cognition (Gregoromichelaki 2013). Consequently, such knowledge needs to be modelled in an architecture that integrates simultaneous qualitatively related constraints from various sources, rather than separate modular components expressed in distinct vocabularies, as the authors advocate. For this reason, we believe the consequences of structural priming, while transparently operative when isolated in carefully controlled experimental designs, seem to disappear in investigations of corpora that reflect multiple other sources of constraints such as frequency, creativity, affective, and social effects (Healey et al. 2014).

In conclusion, an explanation of the structural-priming results from a DS-TTR perspective dispenses with the heterogeneous multilevel representational nature of the grammar proposed by the authors. Yet, this more radical move we propose turns out to be much more supportive of the general conclusion the authors draw, namely, the relevance of psycholinguistic explorations in determining the nature of linguistic theories. It is also more compatible with recent neuro-physiological evidence (e.g., Covington \& Duff 2016). In fact, from our perspective, priming experiments provide valuable tools for guiding the formalisation/implementation of grammar models - for example, by providing measures estimating the temporal course of pattern memory decay, investigating the competition among alternatives resulting in inhibitory effects, and determining variable probability distributions of available sequences, all currently being theoretical and observation-based assumptions in need of further substantiation.

\section{Moving beyond the priming of single-language sentences: A proposal for a comprehensive model to account for linguistic representation in bilinguals}

doi:10.1017/S0140525X17000450, e297

Gerrit Jan Kootstra ${ }^{\mathrm{a}}$ and Eleonora Rossi ${ }^{\mathrm{b}}$

a'Windesheim University of Applied Sciences, 8000 GB Zwolle, Netherlands;

${ }^{b}$ Department of Psychology and Sociology, California State Polytechnic

University, Pomona CA 91768.

g.kootstra.work@gmail.com

gerritjankootstra.wordpress.com

erossi@cpp.edu

sites.google.com/site/eleonorarossishomepage/ 
Abstract: In their target article, Branigan \& Pickering (B\&P) briefly discuss bilingual language representation, focusing primarily on crosslanguage priming between single-language sentences. We follow up on this discussion by showing how structural priming drives real-life phenomena of bilingual language use beyond the priming of unilingual sentences and by arguing that B\&P's account should be extended with a representation for language membership.

In their target article, Branigan \& Pickering (B\&P) argue for structural priming as a key implicit methodology to probe the nature of linguistic representations. They provide extensive data supporting their model, ultimately arguing that structural priming provides a tool to understand the nature of language.

The authors also discuss the implications of their proposal for language representation in bilinguals, focusing primarily on cross-language structural priming between single-language sentences. This research has led to vital insights on cross-language activation at the syntactic processing level (cf., e.g., Hartsuiker \& Pickering 2008), adding to the accumulation of evidence that language use in bilinguals involves ubiquitous cross-language activation at multiple levels of processing (cf. e.g., de Bot et al. 2009; Kootstra et al. 2009; Kroll et al. 2006). Importantly, however, everyday bilingual communication does not normally happen according to a scripted cross-language priming paradigm with primes in one language and targets in the other (cf. Fricke \& Kootstra 2016). To truly understand the nature of language in all its respects, as is B\&P's ambition, we propose that their model and approach should be further developed to explain a larger number of bilingual language scenarios as they occur in real life.

One such bilingual language scenario - and a true hallmark of bilingualism - is code-switching, i.e., the use of multiple languages within one single sentence. Code-switching is susceptible to exactly the same structural priming mechanisms as the production of unilingual sentences, in the sense that bilinguals' syntactic choices in the production of code-switched sentences are primed by those of their dialogue partner (Kootstra et al. 2010). But priming also occurs with dependent variables that are specific to code-switching, namely priming of the sentence position of code-switching (Kootstra et al. 2012), priming of the language of the inflected verb (i.e., matrix language; Fricke \& Kootstra 2016; Kootstra et al. 2010), and priming of the actual choice to code-switch or not (Fricke \& Kootstra 2016; Kootstra et al., in revision). Importantly, these findings are based on both experimental and corpus research. This indicates that structural priming is more than a method to investigate linguistic representations; it is a core mechanism of language use that, together with adaptive control processes (cf., Green \& Abutalebi 2013), appears to guide bilinguals' linguistic behavior in real-life language use.

The critical implication of this code-switching evidence is that B\&P's model should be extended with a representation of language membership. After all, for priming of linguistic elements from multiple languages to take place, these multiple languages must somehow be encoded within the representational system. In most models of bilingual language processing, this is implemented by assuming a language node that is linked to linguistic representations (e.g., Hartsuiker \& Pickering 2008; Kroll et al. 2006). Based on the omnipresence of cross-language activation at all levels of processing, we assume this language node is linked to linguistic representations at all levels of processing (de Bot 2004; de Bot et al. 2009; Kootstra et al. 2009; 2010). Primed code-switching can then be explained in the form of persisting co-activation of language nodes from the recently experienced discourse (see Fricke \& Kootstra 2016; Kootstra et al. 2010).

In addition to code-switching, the just-described extension of B\&P's structural priming account also may serve to explain another fascinating bilingual language scenario: first language (L1) attrition (i.e., loss of or decreased access to L1 representations, mostly due to immersion in a second-language environment, leading to infrequent use of the first language [e.g., Schmid 2011]). Recently, a number of psycholinguistic paradigms have been used to study first-language attrition using offline, online, and neural measures of language comprehension and production (Rossi et al., in revision), but the mechanism of priming so far has not been used to study first-language attrition. Based on B\&P's point that priming can be seen as evidence of access to linguistic representations, it can be predicted that, if L1 representations are completely inaccessible as a consequence of attrition, L1 structural priming should be nonexistent, whereas if L1 representations merely become less accessible as a consequence of attrition, rates of $\mathrm{L} 1$ structural priming may well be relatively strong. This would be consistent with inverse-frequency and surprisal effects found in structural priming studies (e.g., Bernolet \& Hartsuiker 2010; Bock 1986; Ferreira 2003; Jaeger \& Snider 2007; 2013). Another prediction that can be made is that structural priming can serve as a very sensitive measure of changing levels of access to L1 linguistic representations, thus making it possible to boost L1 activation for speakers who are undergoing L1 attrition, much along the lines of what has been proposed for aphasic speakers (Rossi 2013). To continue, under the assumption that structural priming boosts access to linguistic representations by easing the demands on cognitive abilities such as memory, structures that are difficult and/or cognitively taxing should benefit the most from structural priming. Interestingly, these predictions not only show how B\&P's model and its bilingual extension can be utilized to further test existing issues in L1 attrition, but also showcase the intricate relation between structural priming and implicit language learning (e.g., Chang et al. 2006; Dell \& Chang 2014; Ferreira \& Bock 2006).

In sum, we propose to extend B\&P's account with the notion of a language node connected to linguistic representations at all levels of processing. This extended account makes it possible to capture the dynamics of real-life bilingual language use beyond cross-language priming of unilingual sentences, explaining the processes of both cross-language interactivity (e.g., code-switching) and language accessibility (e.g., L1 attrition). Given that more than half of the world's population is bilingual (e.g., Grosjean 2010), this extension is by no means trivial; it is relevant and necessary, and strengthens the generalizability of B\&P's account.

\section{What structural priming can and cannot reveal}

doi:10.1017/S0140525X17000462, e298

\section{Loes Koring $^{\mathrm{a}}$ and Eric Reuland ${ }^{\mathrm{b}}$ \\ ${ }^{\mathrm{a}}$ MIT Linguistics and Philosophy, Cambridge, MA 02139; ${ }^{\mathrm{b}}$ Utrecht Institute of Linguistics OTS, 3512JP Utrecht, Netherlands. \\ koringloes@gmail.com \\ http:/linguistics.mit.edu/user/koring/ \\ eric.reuland@gmail.com \\ https://www.uu.nl//medewerkers/EJReuland}

Abstract: The nature of mental representations of linguistic expressions in relation to the time course from intention to articulation is a major issue. We discuss Branigan \& Pickering's (B\&P's) proposal to use structural priming to tap into this process. We show that their interpretation of their findings cannot be maintained. We reinterpret these results and suggest a revision of their conclusions.

How can we determine the mental representation of linguistic expressions in relation to the time course from intention to articulation and vice versa (Levelt 1989)? A new experimental technique to tap into this process like Branigan \& Pickering's (B\&P's) contribution is very welcome. Their review of priming experiments shows that expressions with a particular linguistic structure can facilitate the use of other expressions with a certain structural similarity. From this, they draw strong conclusions. Their interpretation is not compelling, however, and occasionally reflects a misanalysis (e.g., the Mandarin topicalization in section 2.4 shows only that 
an (A'-bound) empty object is visible for priming). We suggest an alternative interpretation of their findings.

Under mainstream generative accounts, $\mathrm{B} \& \mathrm{P}$ argue, passives involve movement of the underlying object to subject position (leaving an NP trace/copy), whereas intransitive (active) locatives do not. Hence, the two sentence types involve different representations. $\mathrm{B} \& \mathrm{P}$, however, report experiments in which intransitive locatives prime passives. The mainstream account is - they conclude-incompatible with this result.

They also discuss the unergative-unaccusative contrast, which is captured standardly by assuming that the unaccusative argument is first inserted in the DO position and next moved to the canonical subject position. Unergatives don't exhibit such movement. $\mathrm{B} \& \mathrm{P}$ show that, nevertheless, intransitive sentences with unergatives and unaccusatives prime each other. Hence, B\&P argue, their difference is not syntactically represented. Syntactic representations, then, must contain much less detail than generative approaches assume: There is no syntactic movement, and syntactic representations do not contain copies/traces.

However, B\&P mistakenly infer that anything you cannot see with structural priming is "inaccessible" (not used) in processing (sect. 1.1). As is well known, all experimental techniques aren't sensitive to the same processes. If a property established by one technique is not observed with another technique, it is a fallacy to conclude more than that there is a discrepancy to be explained. Crucially, there is abundant evidence that certain properties that, according to $\mathrm{B} \& \mathrm{P}$, are not visible for priming are, in fact, visible to the processor.

For example, B\&P's claim that the contrast between unaccusatives and unergatives is purely semantic and not syntactically encoded is untenable. First of all, the original tests from Perlmutter (1978) and Burzio (1981) show that, unlike the subject of unergatives, the subject of unaccusatives shares syntactic properties with direct objects. Second, these verb types display a difference in processing that is independent of semantic roles (e.g., Agnew et al. 2014; Koring et al. 2012). It follows from a difference in the structural representation, which, consequently, must be visible to the processor, contra $\mathrm{B} \& \mathrm{P}$.

A second misconception concerns their claim that structural priming reveals the exact nature of syntactic representations. As $\mathrm{B} \& \mathrm{P}$ point out themselves, priming displays similarities in representation of a pair of sentences A and B relative to the pair A and C (pp.19-20). As such, this measure cannot tell us directly what the representation of a sentence looks like. A priming effect can tell us at most that particular sentences share some aspects of their representation, but this does not entail that their representations can be identified.

B\&P's appeal to parsimony in their argumentation also fails due to inaccuracies in their exposition (including references to obsolete concepts like Deep Structure). Moreover, the absence of explicit mapping rules between syntax and semantics makes their preferred alternative impossible to assess. In generative theory (see Chomsky 1986; 1995; 2001; also 1955/1975), the role of grammar is not so much to characterize what is grammatical as opposed to ungrammatical, but to characterize the relation between forms and their interpretations. Due to the phase-based organization of derivations, B\&P's reference to levels misses the point. Properties reflecting steps in this derivational process are accessible to the processor, as shown by a variety of experimental techniques currently employed in addition to grammaticality judgment tasks (which B\&P fail to acknowledge) (e.g., Bever \& Sanz 1997; Brennan \& Pylkkänen 2016; Crain \& Thornton 1998; Friedmann et al. 2008; Koornneef et al. 2011; Sprouse et al. 2016).

Unlike what B\&P presume, (Narrow) Syntax is independently characterized, namely as involving operations subject to restrictions (e.g., locality constraints) that are independent of intended meaning. Consider resumptive pronouns in wh-questions. The formation of $w h$-questions is subject to locality conditions. Interestingly, wh-questions that violate a locality condition can be "saved" by using a resumptive pronoun. The resumptive pronoun does not contribute to the meaning but makes an otherwise ungrammatical dependency licit. This shows that the interpretation itself is not blocked, but a particular syntactic derivation to realize that interpretation (for a similar contrast in binding dependencies, see e.g. Reuland 2011a; 2011b; Koornneef \& Reuland 2016). Therefore, B\&P's argument that there is no level of detailed syntactic representation because the priming tool does not track that level is misguided.

Yet, we share B\&P's concern "[to identify] which aspect of structure that priming taps into" (sect. 1.4, para. 7). We suggest that the method of structural priming tracks no more, but also no less, than a particular aspect of detailed linguistic representations-namely, what is visible to the external systems. Phase theory helps us identify this aspect. Phase theory hypothesizes that, once the derivation of a relevant chunk - a propositional structure, a DP/PP - is complete, it is handed over to the realization and interpretation systems. Its internal structure-copies/ traces - becomes inaccessible at that point. Hence, at this handover point, what is accessible in unergative and unaccusative structures will be quite similar, yielding the priming data unsurprising. The same applies to passives. What is visible of their internal structure will lack detail at the handover point, making them sufficiently similar to locatives for priming. Finally, given that scope marking is structurally represented, and the scope marker is external to the core proposition, the latter's internal structure, but not the scope marker, will have become inaccessible at the handover point. This reinterpretation in terms of phases provides a straightforward account of B\&P's findings. In short, phase theory can help understand what structural priming shows.

\section{ACKNOWLEDGMENTS}

We are very grateful to Noam Chomsky, Martin Everaert, Arnout Koornneef, Pim Mak, and Iris Mulders for their very helpful comments on an earlier draft. Any errors are ours.

\section{On the nature of structure in structural priming}

doi:10.1017/S0140525X17000474, e299

\section{Nayoung Kwon ${ }^{a}$ and Yoonhyoung Lee ${ }^{b}$}

${ }^{a}$ Department of English, Konkuk University, Seoul, 143-701, Republic of Korea; ${ }^{\mathrm{b}}$ Department of Psychology, Yeungnam University, Gyeongsan, Gyeongbuk, 712-749, Republic of Korea.

nayoung.kw@gmail.com yhlee01@yu.ac.kr

Abstract: Like Branigan \& Pickering (B\&P), we agree that processing evidence is important for linguistic theorization; however, without much evidence of priming of hierarchical argument structure independent of linear ordering, the nature of "structure" in structural priming remains unclear. Consequently, it is an empirical question whether structural priming and acceptability judgments tap into cognitive processes of a similar nature.

In the Chomskyian tradition, a clear distinction is made between competence and performance (Chomsky 1965), and linguistic theorization has been primarily concerned with native speaker's metalinguistic judgments of sentences. Branigan \& Pickering (B\&P) depart from this tradition and argue that grammar is directly accessed during language processing, so processing evidence is as relevant for linguistic theorization as acceptability judgments are. To be specific, B\&P argue that structural priming can be taken as evidence for linguistic representation. We agree with B\&P that structural priming is a useful tool in the study of language; however, we would like to point out that structural priming also has the issue of "source ambiguity" (similarly to acceptability judgments noted by B\&P; Chomsky 1977), crucially in the context of structural representation assumed in the proposal.

When the processing of input A affects the processing of input $\mathrm{B}$, which shares an aspect of linguistic structure with input A but otherwise is unrelated, the phenomenon is viewed as an instance 
Commentary/Branigan and Pickering: An experimental approach to linguistic representation

of structural priming. What if there is more than one aspect that both input $\mathrm{A}$ and $\mathrm{B}$ share? In this case, the source of the priming effect remains ambiguous. Unfortunately, most evidence taken in support of structural priming has this source ambiguity issue - in particular, ambiguity between sequential and hierarchical relations. For example, although structural priming effects were reported in many studies of structural alternations without meaning change-e.g., alternations between prepositional/ double-object sentences (henceforth, POs/DOs), passive/active sentences, different orderings of the auxiliary and main verb, and different positions of a particle in phrasal verbs (Bock 1986, 1989; Hartsuiker \& Westenberg 2000; Konopka \& Bock 2009; Messenger et al. (2012b); Pickering \& Branigan 1998), the prime and target sentences in these studies shared both linear ordering and hierarchical argument structure (cf. Hare \& Goldberg [1999] for discussion of potential semantic influences). Thus, in these cases, it is not clear whether structural priming effect arises due to linear or hierarchical relations.

$\mathrm{B} \& \mathrm{P}$ ambiguously state that syntactic representations that they assume are "shallow" and "monostratal" such that they "represent hierarchical and linear relations simultaneously" (sect. 2.1, para. 7, 8). Under this assumption, the priming effects found with PO/DO or passive/active alternations above would not have "source ambiguity" as distinction of linear vs. hierarchical relations becomes irrelevant, a notion with which we do not agree. An alternative account, however, is that priming is sensitive to cognitive computations of linear relations but may not be so sensitive to hierarchical relations of linguistic representation. Under this hypothesis, the priming evidence with the potential source ambiguity discussed above is accounted for straightforwardly in terms of priming of linear ordering, which is also consistent with the findings of Pickering et al. (2002), in which sentences that share hierarchical but not linear relations did not prime each other.

Word order is closely related to hierarchical argument structures; however, we believe that these two cannot be equated. Take sentences with a reflexive (e.g., "John ${ }_{i}$ told $\mathrm{Tom}_{k}$ to be kind to himself $_{{ } i / k}$ " vs. "John ${ }_{i}$ seemed to Tom $_{k}$ to be kind to himself ${ }_{i /{ }^{\circ} k}$; cf. Sturt \& Kwon 2015). Although local proximity is a factor, these examples clearly illustrate that the proximity is defined in terms of hierarchical relations and not linear ordering (Reinhart 1983; cf. Langacker 1969). Thus, syntax cannot be reduced to simple sequential structure, and hierarchical relationships are an integral aspect of human language syntax. As such, we believe that we need clear evidence in support of priming of hierarchical relations for the proposal of B\&P to work (cf. Scheepers et al. 2011).

Thus, while structural priming seems convincing with many replications in various languages and participant populations, its nature remains unclear, and therefore, the use of priming experiments in lieu of (or alongside) acceptability judgments is a limited approach to understanding grammatical structure. The proposed approach would benefit greatly from experimental results using various syntactic constructions with which the priming of hierarchical structure can be clearly evaluated independently of linear ordering. In short, clearer evidence of priming of hierarchical argument structure as well as word order is necessary before it can be argued that priming paradigms can be used to answer questions of structure, a core feature of human language syntax.

\section{Considering experimental and observational evidence of priming together, syntax doesn't look so autonomous}

doi:10.1017/S0140525X17000486, e300

Nicholas A. Lester, John W. Du Bois, Stefan Th. Gries, and Fermín Moscoso del Prado Martín

Department of Linguistics, University of California, Santa Barbara, Santa Barbara, CA 93106. nlester@umail.ucsb.edu dubois@linguistics.ucsb.edu

http://www.linguistics.ucsb.edu/faculty/dubois/

stgries@linguistics.ucsb.edu

http://www.linguistics.ucsb.edu/faculty/stgries/

fmoscoso@linguistics.ucsb.edu

http://www.linguistics.ucsb.edu/people/fermin-moscoso-del-prado-martin

Abstract: We agree with Branigan \& Pickering (B\&P) that structural priming experiments should supplant grammaticality judgments for testing linguistic representation. However, B\&P overlook a vast (corpus-) linguistic literature that converges with - but extends - the experimental findings. B\&P conclude that syntax is functionally independent of the lexicon. We argue that a broader approach to priming reveals cracks in the façade of syntactic autonomy.

Branigan \& Pickering $(\mathrm{B} \& \mathrm{P})$ make a compelling case for the utility of experimental methods - in particular, priming - for understanding linguistic representation. We whole-heartedly support this position. As linguists, however, we must note that B\&P have misrepresented the state of affairs within linguistics. The claim that linguists rely solely (i.e., "on a single method," "dominantly," "almost exclusively") on acceptability judgments is an exaggeration. Said judgments have indeed been prevalent in the work of some linguists, but - especially in the last two decades - this is far from the sole method used. A glance at the papers forthcoming in Language finds just one paper using acceptability judgments, but four using analyses of observational data or corpora and two using advanced statistical techniques. Furthermore, several major schools of linguistic thought have flatly rejected the validity of acceptability judgments for more than three decades (e.g., Bybee 2006; Chafe 1994; Givón 1983; Thompson \& Mulac 1991).

Much of what we discuss below relies on corpus data. Pickering and Branigan (1999) argue that such data cannot speak to the nature of priming, given their relatively low level of control compared to well-controlled experimental designs. This assumption reflects a common prejudice among experimental psychologists: That the "found data" nature of corpora makes them unsuitable for disentangling target effects from confounds. Modern statistical techniques now enable distinguishing the influences of many confounding variables. In fact, many variables important to priming are more difficult to control for in experimental paradigms than in corpus studies (e.g., distance effects between prime and target, beta-persistence [Szmrecsanyi 2006]; effects of non-variable structures on variable contexts, cumulative priming effects [Jaeger \& Snider 2013]).

$\mathrm{B} \& \mathrm{P}$ argue that syntactic representations are independent of semantics and lexicon. This assertion is ambiguous (Croft 1995). We all agree that syntactic aspects are (explicitly or implicitly) represented in the mind. However, saying that syntax is functionally and/or representationally encapsulated apart from lexicon and semantics is more contentious. B\&P support this claim by showing that abstract clausal templates (e.g., prepositional-object [PO] or double-object [DO]) are primed even without lexical overlap between the prime and target. Further, semantically dissimilar but syntactically similar structures prime each other. For example, intransitive + locative-PP constructions prime passives. However, these intransitives and passives have more in common than acknowledged by the authors. For instance, ergative languages align such structures along both syntactic and semantic dimensions (Keenan 1984). Moreover, semantic similarity beyond lexical overlap has been found to drive syntactic choice of PO/DO, even in the absence of syntactic similarity (Hare \& Goldberg 1999).

Another strong indicator of the semantic properties of clausal constructions is the statistical association between verbs and constructions (Goldberg 2006; Ellis \& Ferreira-Junior 2009; Stefanowitsch \& Gries 2003). These associations co-determine the magnitude of priming (e.g., Gries 2005). Importantly, they do not merely boost priming but may actually resist priming (and these relationships may change depending on context [Jaeger \& Snider 2013]). Lexical choices also often dictate syntactic choices, both in production and in comprehension (e.g., Jaeger 
2010; Novick et al. 2003). Importantly, such choices may be influenced by syntactic information that prima facie should be irrelevant to the syntactic alternation under consideration (Wasow et al. 2011). Furthermore, words - even syntactically impoverished bare nouns - are never processed in isolation from the entirety of their syntactic distributional information, and may prime each other via such distributions (Lester \& Moscoso del Prado Martín 2016; Lester et al. 2017).

B\&P survey clear evidence of priming among words, syntactic structures, and semantic structures. They also explain how simultaneous overlap between any two of these levels results in increased priming (the so-called boosts). One can account for these findings in two ways: (1) relationships among syntax, semantics, and lexicon are captured by additional interfaces whose only job is to combine information from separate modules (e.g., Jackendoff 2013); or (2) the relationships constitute connection weights between words and structures, which are directly related in memory (Diessel 2015). B\&P appear to prefer the first option. However, short of undisputed neuropsychological evidence for the separation between these representational levels (which is not known to us), there is no way of distinguishing among three separated levels with connections between them, and a single level of representation with different degrees of overlap. Considering that priming effects are very similar in the three levels, and that overlap among them interacts, it seems more parsimonious to assume a single layer of representation, rather than positing three such encapsulated layers plus interconnections.

B\&P's arguments rely on binary choices (such as PO/DO). However, it is unlikely that these choices could benefit from structural overlap in phrasal constituents; the critical variable depends only on where those phrases are placed. If there is no additional reason to adjust structures to accommodate the accessibility of subclausal units, then why would one? Whether there may be a taskdriven confound remains a question for further study. However, notice that chronometric studies show that the locus of priming may not always be the clause, even when clause-structural overlap is present (Smith \& Wheeldon 2001). Further, more comprehensive models of linguistic reproduction exist, which make distinctions beyond simple identity priming. Consider Dialogic Syntax (Du Bois 2014; Du Bois et al. 2014), which distinguishes among framing resonance, the locus of syntactic priming, and focal resonance, the aligning of meanings within syntactic alignment.

We emphasize that we are not advocating the position that syntactic priming is reducible to lexical, semantic, or pragmatic effects. To truly understand linguistic representation on the basis of processing, we must consider all possible sources of information from processing across all levels that are brought to bear on language use, including data from both experimental and observed contexts. This trend is already well underway in several major branches of linguistics. B\&P's bold proposal to establish "a new basis for understanding the nature of language" stands to benefit from a full partnership with researchers drawing on a broad range of evidence to account for a system that dynamically responds to linguistic, cognitive, and interactional contexts.

\section{Structural priming, action planning, and grammar}

\author{
doi:10.1017/S0140525X17000498, e301
}

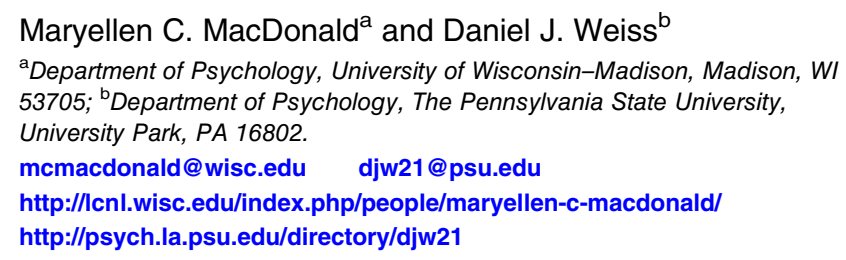

Abstract: Structural priming is poorly understood and cannot inform accounts of grammar for two reasons. First, those who view performance as grammar + processing will always be able to attribute psycholinguistic data to processing rather than grammar. Second, structural priming may be simply an example of hysteresis effects in general action planning. If so, then priming offers no special insight into grammar.

Branigan \& Pickering (B\&P) argue that structural priming methods have "reached maturity" (target article, para. 2) to the point that they can inform not only language production and comprehension processes, but also the nature of grammar, as typically studied by linguists using different analytical tools and methods. This view appears overly optimistic; structural priming remains widely used but poorly understood, with little consensus about why the effect is observed or exactly what production and comprehension processes are promoted from prior exposure to a sentence. Moreover, the larger class of priming methods, to which $\mathrm{B} \& \mathrm{P}$ link structural priming, has been the target of extensive criticism and reassessment of what can be gleaned from the tasks (Cesario 2014). Here, we consider two perspectives on the nature of structural priming and their consequences for B\&P's claims for grammar.

One perspective is that structural persistence is a strongly syntactic phenomenon: Encountering/producing a sentence somehow biases the language processing system to expect or produce a similar syntactic structure. B\&P's logic is that, because the processing system draws on the grammar, patterns of priming must reveal the nature of the grammar. This thinking raises the classic issues of the competence-performance distinction. If language use is grammar + processing, there is a credit assignment problem for psycholinguistic data: Any linguistic behavior might reflect the grammar, processing mechanisms, or some combination. B\&P make exactly this criticism of other psycholinguistic methods - for example, that Franck et al.'s (2010) studies of subject-verb agreement production might illuminate the nature of the grammar, or alternatively they might reflect production or comprehension processes and be uninformative about grammar. Crucially, this assignment problem applies equally to priming. Haskell et al. (2010) used priming to study agreement production and found that subject-verb agreement is sensitive to the statistical patterns in prior usage (the primes). These results could support a graded grammar in which statistical patterns shape grammatical representations (Bybee 2006). Researchers rejecting this approach, however, could instead attribute these priming data to processing, leaving the grammar untouched by the statistics of usage. Thus, given B\&P's assumption of usage=grammar + processing, structural priming is just as much subject to interpretive uncertainty as any other measure.

Even more interpretive uncertainty arises from an alternative view of structural priming - that it is not strictly syntactic but rather a language example of a more general tendency to repeat prior actions. Cognitive models of motor planning suggest these reuse effects (termed hysteresis effects) arise because it is easier to recall a previously executed motor sequence than to generate alternative plans de novo (Rosenbaum et al. 2006). Our own research investigates the link between structural priming and domain-general plan reuse, and we have developed parallel tasks that yield reliable structural priming for dative sentence structures and priming of nonlinguistic manual actions in the same participants (Koranda et al. 2016). We also observed a parallel effect of priming strength in both domains: Preferred sentences and movements are more easily primed than unpreferred ones, a phenomenon previously observed in structural priming (Bock 1986). These findings raise the possibility that plan reuse may be a domain-general property of action planning. MacDonald (2013) suggested that a general plan reuse bias would ground patterns of language use in basic planning mechanisms, and the existence of a general plan reuse bias may also explain why some nonlinguistic motor sequences such as stacking blocks appear to prime sentence structure choices in language 
production or comprehension (Allen et al. 2010; Kaiser 2012). On this domain-general view, sequences in one type of action may potentiate an analogous sequence in another domain under certain task demands (Van de Cavey \& Hartsuiker 2016). Clearly, the space of such domain-general priming effects is currently poorly understood, but if structural priming proves to be emergent from broader components of action planning, then there is little reason to expect that the phenomenon offers privileged insight into grammar.

Further investigation of the domain specificity versus generality of plan reuse will therefore be critical for gaining insight not only into priming as a tool, but also into the forces that shape implicit action choices, including, but not limited to, choices of syntactic structure. The mechanisms supporting plan reuse are likely to be highly conserved across domains and species, given that nonhuman primates exhibit homologous hysteresis effects (see Weiss \& Wark 2009). A signature characteristic of hysteresis is asymmetry, such that a transition point between implicit action choices varies with prior history. For example, in studies in which reaching targets shift gradually clockwise or counterclockwise across trials, both human children and tamarin monkeys transition from left- to right-hand use at different points depending on past targets (Rostoft et al. 2002; Weiss \& Wark 2009). Our ongoing work investigates whether similar perseverative asymmetries are found in both motor and language production tasks with gradual changes in parameters that promote one versus another hand/syntactic choice. If so, these findings would suggest that structural priming is a subcategory of a broader cognitive heuristic. A related opportunity to study the domain-general versus specific nature of planning is the investigation of individual differences in plan reuse across domains. For example, working memory tasks are a classic locus of individual differences in cognitive performance, and both spatial and verbal working memory loads appear to interact with hysteresis effects in motor planning (Spiegel et al. 2013). Such interactions are most consistent with a domain-general planning system, and individual differences in these interactions should further constrain theoretical accounts and also inform our understanding of priming. Indeed, individual differences in structural priming (Kaschak et al. 2011a; Kidd 2012) seem inconsistent with B\&P's claims that priming reveals grammar, which is conceived as an abstract representation with only trivial variation across a language community. As we learn more about priming, we suspect that the lessons for grammar will not be the ones $\mathrm{B} \& \mathrm{P}$ promote but instead will suggest that the nature of domain-general action planning has an important role in patterns of syntactic structures in language use.

\section{Structural priming is most useful when the conclusions are statistically robust}

\author{
doi:10.1017/S0140525X17000504, e302
}

\section{Kyle Mahowald, ${ }^{\mathrm{a}}$ Ariel James, ${ }^{\mathrm{b}}$ Richard Futrell, ${ }^{\mathrm{a}}$ and Edward Gibson ${ }^{a}$}

${ }^{a}$ Department of Brain and Cognitive Sciences, Massachusetts Institute of Technology, Cambridge, MA 02139; ${ }^{\mathrm{b}}$ Psychology Department, University of Illinois at Urbana-Champaign, Champaign, IL 61820.

kmahowald@gmail.com anjames2@illinois.edu futrell@mit.edu egibson@mit.edu

\begin{abstract}
Branigan \& Pickering $(\mathrm{B} \& \mathrm{P})$ claim that the success of structural priming as a method should "end the current reliance on acceptability judgments." Structural priming is an interesting and useful phenomenon, but we are dubious that the effect is powerful enough to test many detailed claims about specific points of syntactic theory.
\end{abstract}

Branigan \& Pickering ( $\mathrm{B} \& \mathrm{P})$ claim that the success of structural priming as a method should "end the current reliance on acceptability judgments." The basis of such a claim rests on the premise that, not only is structural priming a robust psychological phenomenon, but also can be used to adjudicate between finer points of syntactic theory. While we agree that structural priming is an interesting and useful phenomenon, we have reservations as to whether there is sufficient statistical evidence to support all of the detailed claims made about specific points of syntactic theory.

For example, as a case study of what they call the "clearest example," B\&P discuss what priming studies tell us about passive constructions and, specifically, how priming studies push back against the standard generative grammar accounts of passives. The details of this argument rely on priming studies that go beyond asking whether Structure $\mathrm{X}$ in a prime sentence leads to an increased likelihood of a participant producing Structure $\mathrm{X}$ in a target sentence. Rather, it relies on evidence that locatives prime passives (Bock \& Lobell 1990), that unergatives prime unaccusatives (Flett 2006), and that POs and DOs prime light verbs (Wittenberg 2014). Often, there is only one paper that investigates a particular research question, and B\&P typically accept the results of that paper as delivering a statistically valid conclusion about the phenomenon in question.

In a meta-analysis in Mahowald et al. (2016b), we give evidence that structural priming is a robust and well-replicated phenomenon, but caution that studies of the type that B\&P rely on for some of their conclusions (specifically, studies that ask whether some priming effects are greater than others or whether certain factors, such as age or L2 status, moderate priming effects) are statistically underpowered (with an average power of $53 \%$, as determined by a p-curve analysis) and often do not use enough participants to warrant the conclusions drawn. Indeed, in studies of this sort, we recommend using several hundred participants - which almost no existing priming studies do.

While this is not evidence that the conclusions drawn in any one study that B\&P referenced are misleading, we should be cautious not to assume that every individual study can be used as a building block in a larger syntactic theory. Rather, we should expect some studies to reach statistically significant conclusions (or fail to reach statistically significant conclusions) based on chance alone - and not just because of the experimental manipulation. This is of particular concern when there is only one unreplicated study on a particular phenomenon.

Of course, none of this is to say that structural priming should not be used alongside and sometimes instead of acceptability judgments (which have their own host of pitfalls). In the often messy world of empirical science, the availability of diverse, orthogonal methods that explore the same research question using different techniques is a feature, not a bug. So, insofar as $\mathrm{B} \& \mathrm{P}$ argue against the hegemony of one particular technique (acceptability judgments) in linguistics research, we agree. Insofar as they argue for the hegemony of a different technique (priming), we urge a healthy skepticism.

\section{Priming methods in semantics and pragmatics}

doi:10.1017/S0140525X17000516, e303

\section{Mora Maldonado, ${ }^{\text {a,b }}$ Benjamin Spector, ${ }^{a}$ and Emmanuel Chemla ${ }^{\mathrm{b}}$}

a'Institut Jean Nicod, Département d'Études Cognitives, École Normale Supérieure, PSL Research University, CNRS, EHESS, 75005 Paris, France; ${ }^{\mathrm{b}}$ Laboratoire de Sciences Cognitives et Psycholinguistique, Département d'Études Cognitives, École Normale Supérieure, PSL Research University, CNRS, EHESS, 75005 Paris, France.

mora.maldonado@ens.fr benjamin.spector@ens.fr

chemla@ens.fr

http://mmaldonado.psycholinguae.fr/

https://sites.google.com/site/bspectorpage/

http://www.emmanuel.chemla.free.fr/ 
Abstract: Structural priming is a powerful method to inform linguistic theories. We argue that this method extends nicely beyond syntax to theories of meaning. Priming, however, should still be seen as only one of the tools available for linguistic data collection. Specifically, because priming can occur at different, potentially conflicting levels, it cannot detect every aspect of linguistic representations.

Branigan \& Pickering $(\mathrm{B} \& \mathrm{P})$ argue that structural priming is a powerful method to inform theories of linguistic structure, and they even suggest that it could supersede other methods such as acceptability judgments. We will argue that the method extends nicely beyond syntax to theories of meaning, in which priming can serve to reveal abstract interpretive operations. In doing so, however, we will see why structural priming should still be seen as one among many of the tools available for linguistic data collection. In particular, because priming can occur at different, potentially conflicting levels, it cannot detect every aspect of linguistic representations.

The primary data used in formal semantics/pragmatics are truth-value and inferential judgments. These methods document the result of interpretive processes: what a sentence ends up meaning. Priming methods can be useful here just as in syntax: Schematically, acceptability judgments target the output of a cognitive process, and priming data may offer a window into some aspects of this process. In formal semantics and pragmatics, the relevant elementary interpretive processes are often abstract "invisible" operations. Here are two prime examples:

1. A silent distributivity operator whose meaning is akin to each has been postulated to explain why sentences involving more than one plural expression, such as Two boys read three books, have both a cumulative interpretation (i.e. Two boys read three books in total) and a distributive interpretation (i.e. Two boys read three books each). See Champollion (in press), for a survey.

2. Sentences such as Some of the students came tend to acquire a strengthened meaning amounting to Some but not all of the students came (scalar implicature). On some accounts, this strengthening is a pragmatic process, while on others, it is due to the presence of a covert exhaustivity operator. In both approaches, however, the very same mechanism is responsible for the strengthening of some into some but not all, may into may but does not have to, three into exactly three (though this last case is more controversial).

Distributivity and exhaustivity operators are often thought of as covert linguistic operators, which are part of syntactic representations (Chierchia et al. 2012; Link 1987). Alternatively, exhaustivity operators also can be seen as precompiled proxies for late, postcompositional pragmatic processes (Spector 2007; van Rooij \& Schulz 2004). In either case, the possibility of priming exhaustive or distributive interpretations across sentences featuring different trigger words would confirm the posited abstract mechanisms, beyond their mere interpretive effects. Indeed, recent studies using typical priming paradigms (e.g., Raffray \& Pickering 2010) have provided evidence in favor of an abstract mechanism for distributivity (Maldonado et al. 2017 ) and exhaustivity (see Bott \& Chemla 2016; Chemla \& Bott 2014; Rees \& Bott, submitted): schematically, the presence of a distributivity/exhaustivity operator in a sentence primes the presence of this operator in a subsequent distinct sentence, showing that the cognitive representations associated with both sentences share an abstract property.

Because priming can occur at different levels (e.g., syntax and semantics), it is tempting to assume that every aspect of linguistic representations can be primed. From there, B\&P suggest that we could use the absence of priming effects triggered by certain hypothetical operations as an argument against theories that posit such operations. This is too radical, however-as we will briefly argue, focusing on scope assignment in doubly quantified sentences (B\&P; Chemla \& Bott 2015; Feiman \& Snedeker 2016; Raffray \& Pickering 2010). First, a specific operation can be well motivated independently of priming. Second, the absence of a particular priming effect may be due to a conflict with another potential source of priming.

The Quantifier Raising (QR) operation has been hypothesized to explain cases of mismatch between the surface ordering of quantifiers and their relative semantic scope: the sentence A girl invited every boy can receive the interpretation For every boy $x$, $a$ girl $y$ invited $x$, in which the universal quantifier takes scope above the existential, reversing the surface ordering at the interpretive level. Importantly, QR is not the only possible mechanism to derive inverse scope (through movement). Crucially, all frameworks, including the Parallel Architecture view (Culicover \& Jackendoff 2005) endorsed by B\&P, need to characterize the mapping between syntax and semantics, and therefore need some mechanism to account for inverse scope interpretations.

Given that semantic operations can be primed (cf. above), the inverse-scope operation (whatever it is, under any account) might be primed in principle. As observed in the target article, recent studies (Chemla \& Bott 2015; Raffray \& Pickering 2010) did not observe priming of the inverse-scope operation. Instead, these studies revealed priming of the scopal relation itself: A sentence interpreted with a universal quantifier taking scope over an existential quantifier would prime a similar interpretation of a subsequent sentence, with a universal taking scope over an existential quantifier, regardless of whether these interpretations require inverse-scope of the prime sentence and/or of the target. Priming of the inverse scope operation was not found, but this potential priming effect was pushing in a direction opposite to that of priming of relative scope. The only conclusion we can draw, then, is that the latter is stronger than the former, and certainly not that an inverse scope mechanism does not exist (especially given that such a mechanism is necessary in any framework to account for interpretive judgments).

Priming can be used to reveal the existence of a (primable) aspect of linguistic representations, in syntax and in semantics as we have shown, but not so much to argue against the existence of a (less primable or potentially non-primable) feature. Linguistic theories should (a) represent the primable features, and (b) provide the means to distinguish between more or less primable features. Although (a) is consistent with B\&P, one may understand $\mathrm{B} \& \mathrm{P}$ as implying that all aspects of linguistic representations in principle can be equally primed, thus rejecting (b). Such a radical view might lead to an unwarranted bias in favor of less expressive theoretical frameworks, by allowing researchers to ignore all aspects of linguistic phenomenology that are not detectable in priming experiments.

\section{ACKNOWLEDGMENTS}

We wish to thank Tal Linzen for useful comments and discussion. The research leading to these results has received funding from the European Research Council under the European Union's Seventh Framework Programme (FP/2007-2013)/ERC Grant Agreement n.313610 and from the Agence Nationale de la Recherche (Grants ANR-10-LABX-0087 IEC, ANR-10-IDEX-0001-02 PSL* and ANR-14-CE30- 0010-01 TriLogMean).

\section{Can structural priming answer the important questions about language?}

doi:10.1017/S0140525X17000528, e304
Andrea E. Martin, ${ }^{a, b}$ Falk Huettig, ${ }^{a}$ \& Mante S. Nieuwland ${ }^{a}$
aMax Planck Institute for Psycholinguistics, 6525 XD Nijmegen, Netherlands; ${ }^{\mathrm{b}}$ School of Philosophy, Psychology, and Language Sciences, University of Edinburgh, Edinburgh EH8 9JZ, United Kingdom.
andrea.martin@mpi.nl falk.huettig@mpi.nl
mante.nieuwland@mpi.nl
https://sites.google.com/site/aemn1011/
http://www.mpi.nl/people/huettig-falk
http://www.mpi.nl/people/nieuwland-mante 
Abstract: Structural priming makes a valuable contribution to psycholinguistics, but it taps into implicit memory representations and processes that may differ from what is deployed during online language processing. As a result, the strength of inductive inference regarding linguistic representation is rather limited. We question whether implicit memory for language can and should be equated with linguistic representation or with language processing.

Branigan \& Pickering $(\mathrm{B} \& \mathrm{P})$ assert that structural priming reveals the nature of linguistic representation and does so over and above other available psycholinguistic methods -acceptability judgments in particular. We wholeheartedly agree with B\&P on the limitations of acceptability judgments, and on structural priming being an interesting phenomenon that is of use to language researchers. However, if the scientific aim is to study linguistic representation, we argue that the structural priming paradigm is also limited in many important ways because of its reliance on domain-general implicit memory representations and processes, and because it only allows unidimensional inferences.

Priming is a form of implicit learning that stems from implicit memory formed during recent processing (sect. 1.4), measured through its effect on current processing. This begs the question whether structural priming can indeed separate representation from process. $\mathrm{B} \& \mathrm{P}$ assume that priming reflects changes in availability of the representations needed for processing, but that processing itself is somehow not affected (sect. 1.4, para. 8). We think that this assumption is untenable. Very little is known about the implicit memory of recent language processing, the representations that underlie priming. How stable are these representations? How do they relate to the representations formed during online language processing? Are they implemented in language-specific and/or domain-general memory processes? It seems these questions are ignored at our peril if structural priming is to be a method for understanding linguistic representations. B\&P claim to work toward a theory of linguistic representation, but the domain-general nature of priming does not allow inferences about whether representations are linguistic or non-linguistic. In our view, structural priming is best seen a more limited experimental approach to understanding implicit memory for language. Whether that suffices as an approach to linguistic representation we leave to the reader.

As an experimental approach, structural priming allows for rather limited inferences. One problem is that it suffers from the same source ambiguity and response bias (sect. 1.2) that confounds other behavioral measures (e.g., two-alternative forced choice or reaction time measures; see Macmillan \& Creelman 2004; Martin 2016; McElree 2006). The relationship between representation and process necessarily is blurred in measurements like these, because participants can trade speed for accuracy, and vice versa, using an internal criterion that can be related to either representational quality, bias, or the time it takes for a process to occur. Unlike techniques such as speedaccuracy tradeoff modeling (McElree, 2006; Reed 1973), structural priming cannot tease apart effects stemming from representational quality and those from processing speed.

Another problem with structural priming is that it allows only unidimensional inferences (count in production, RT in comprehension), so we can observe only "greater than" and "less than" effects. This is problematic because observing similar priming effects (i.e., null results, sect. 2.4, para. 2) does not necessitate the conclusion that underlying representations are similar, and observing different priming effects do not necessitate the conclusion that underlying representations are inherently different. Furthermore, because structural priming is not time-resolved, nothing can be learned about when linguistic representations are used, or about how these representations change over time. Moreover, structural priming (in production at least) is limited to sentence structures that have an alternative structure describing the same event approximately equally well, and therefore has very limited scope in terms of what can be tested.
Some of these issues can be overcome with neuroimaging techniques such as ERPs and fMRI. B\&P discount these techniques because of a lack of one-to-one mapping between the measure of brain activity (ERP component or localized brain activity) and levels of linguistic representation. We think that this is both unfair and misguided. Recent neuroimaging findings suggest that semantic and syntactic levels of representation are inextricably linked in processing (e.g., Nieuwland et al. 2013) and that linguistic representations are implemented in a dynamically-bound network configuration (e.g., Hagoort 2014; Skipper 2015; for modelling evidence see Martin \& Doumas 2017). However, this does not disqualify neuroimaging as a general method to study the processing of linguistic representations, especially with the advent of new decoding techniques (King \& Dehaene 2014), it merely shows that the actual implementation of linguistic processes and representations in the human brain is very complex and not sufficiently understood.

In sum, we challenge the claim that "evidence from structural priming supports quite specific proposals about linguistic structure" and question the extent to which it "can be used to develop linguistic theory and discriminate among competing accounts" (para. 2), mostly because we question the definition of a linguistic representation in the context of structural priming. As far as we can ascertain, it is an implicit memory representation with an indeterminate relationship to online representation and subsequent processing.

Our deeper concern is that priming doesn't explain how representations, either in production or in comprehension, are formed in the first place, nor how language processing unfolds and produces meaning. Furthermore, we think that the mechanism through which activation of comprehended structure influences produced structure is at stake for the theoretical advances that the authors are interested in, and we believe that progress towards a truly mechanistic theory of language cannot be made until processing mechanisms are formalized and computationally specified. Only then can the interaction between representations and processes during language use begin to be understood.

\section{Structural priming is not a Royal Road to representations}

\author{
doi:10.1017/S0140525X1700053X, e305
}

\section{Antje S. Meyer \\ Max Planck Institute for Psycholinguistics and Radboud University, 6500AH Nijmegen, Netherlands. \\ antje.meyer@mpi.nl \\ www.mpi.nl}

Abstract: Branigan \& Pickering (B\&P) propose that the structural priming paradigm is a Royal Road to linguistic representations of any kind, unobstructed by influences of psychological processes. In my view, however, they are too optimistic about the versatility of the paradigm and, more importantly, its ability to provide direct evidence about the nature of stored linguistic representations.

I agree with the authors that the way language is represented in the human mind is a central question for the language sciences; that the question must be studied empirically; that obtaining acceptability judgements from experts or laypersons is not sufficient to do so; and that important evidence about the nature of linguistic representations can be gleaned from structural priming (SP) experiments. The fruitfulness of the paradigm is amply illustrated in the theoretical part of the target paper and by the papers in Dell and Ferreira (2016).

I am not convinced, however, that the paradigm is as versatile as the authors suggest. Designing and running SP experiments is not trivial. As Mahowald et al. (2016b) show in a meta-analysis, SP 
effects are usually small to moderate, and for adequately powered designs, many items and/or participants are needed. This limits the usefulness of the paradigm for studies involving children and patients. Moreover, for secure interpretation of SP effects, many conceptual and linguistic variables (frequencies of words and word combinations, plausibility, etc.) must be taken into account; for production experiments, suitable ways of reliably eliciting the target utterances must be found.

Of course, similar considerations hold for other paradigms. One issue, however, pertains specifically to $\mathrm{SP}$ - in particular, to the canonical variant of the paradigm in which the speakers' choices of grammatical structures are recorded. As the authors discuss, the design of SP experiments requires the existence of pairs of roughly equivalent structures (e.g., active and passive) for participants to choose between. Therefore, it is challenging to use SP in research on properties of grammatical representations, such as subject-verb agreement or fixed word order (e.g., in English polar questions), for which no alternatives exist in the language. Thus, the authors' statement that "priming can be used similarly to investigate the representation of any aspect of linguistic structure" (sect. 1.4, para. 4) seems overly optimistic.

I am also not convinced that, as B\&P propose in section 1.4 of the target paper, "priming effects arguably implicate a direct relationship between representation and behavior" (sect. 1.4, para 2; see also sect. 1.5, para. 1: "It [structural priming] provides evidence that is directly informative about mental representations"). They propose that comprehension-to-production priming reflects on shared representations rather than processing components specific to production or comprehension; they also argue that SP effects differ from judgements, chronometric, or neurobiological data collected in other paradigms by being uncontaminated by processing influences: "It is hard to see how the explanation of priming could depend on processing assumptions" (sect. 1.4, para. 9). The authors acknowledge that priming may occur for reasons other than similarity of representations, but they believe that such effects should be easy to identify through careful experimentation.

It is not clear to me why comprehension-to-production priming necessarily reflects on shared representations rather than shared processes, and why the authors believe that it is easy to separate effects of the similarity of representations from other causes of priming. They point out that priming effects occur "without awareness or explicit recall of the prime stimulus and are generally believed to be automatic and resource free" (sect. 1.4, para. 2). However, processes that occur without awareness and that are largely automatic are still processes. Most important, these processes change the speaker's language use: After presentation of a prime, the primed structure is more readily available than it was before. Thus, SP effects do not only reflect on the degree of similarity between linguistic representations or processes, but also on their malleability. This is why SP often is seen as a form of implicit learning (e.g. Bock \& Griffin 2000; Chang et al. 2006).

In addition to changing the availability of known structural alternatives, SP can introduce new constructions into a person's repertoire. For instance, in a reading study, Fraundorf and Jaeger (2016) showed priming for sentences such as The car needs cleaned, which featured a structure (need + past participle) that was not part of the participants' dialect. This effect generalized to structurally similar sentences featuring will + past participle, as in The copier will recycled. Interestingly, facilitation for will sentences after exposure to need sentences occurred only in participants who were not familiar with the need + past participle construction before the experiment but not in participants whose dialect permitted this construction. Fraundorf and Jaeger speculated that this pattern arose because only the former group expected encountering unfamiliar structures. Whatever the merits of this specific account, the results illustrate, first, that SP can alter a person's linguistic repertoire, and, second, that such changes are subject to multiple influences, including the person's expectations about the utterances likely to occur in the current context. This latter conclusion is supported by several other recent studies (e.g., Myslín \& Levy 2016). Fraundorf and Jaeger reported a comprehension study. Maybe speakers' choices in production experiments are less readily affected by other variables than prime-target similarity. It seems highly unlikely, however, that they are entirely impervious to such influences, or, as B\&P suggest, that it is trivial to separate them from structural similarity effects.

In sum, the claim that SP offers an unobstructed route to linguistic representations seems incorrect to me. SP shows how grammatical choices change with experience. Choices are behaviour, and like any other type of complex behaviour, they are based on stored knowledge and cognitive processes using this knowledge. The authors note that acceptability judgements suffer from "source ambiguity" - uncertainty about the origins of observed effects. Exactly the same holds for SP and, in fact, any other psycholinguistic

\section{The syntax of priming}

doi:10.1017/S0140525X17000541, e306

\section{William O'Grady \\ Department of Linguistics, University of Hawaii at Manoa, Honolulu, HI 96822. ogrady@ hawaii.edu \\ http://ling.hawaii.edu/william-ogrady/}

Abstract: Priming reflects the reactivation of processing routines that map strings of words onto semantic representations (and vice versa) without the mediation of syntactic structure, including the "flat structure" that Branigan \& Pickering $(\mathrm{B} \& \mathrm{P})$ propose. Key evidence for this claim comes from the possibility of priming relations involving subject-verb sequences, which are not syntactic constituents.

Language provides a way to map meaning onto form and vice versa. We know, from direct observation, that the form side of the mapping consists of a string of words, inflected in some languages and bare in others, arranged in a particular order. And we know, from inference, that the meaning side includes at its core a representation of a predicate and its argument(s), along with ways to express information-related contrasts and scopal relations.

We do not know how form and meaning are related to each other. It is generally assumed that the relationship is mediated by a syntactic representation that organizes words into everlarger hierarchically organized constituents. Drawing on the hypothesis that priming is sensitive to constituent structure, Branigan \& Pickering (B\&P) argue for a comparatively flat syntactic structure, as illustrated in Figure 1.

Another idea, common in work on cognitive science, proposes a direct mapping between strings of words and semantic representations, mediated by processing operations that make no reference to traditional syntactic structure (e.g., O'Grady 2015). On this view, a sentence such as Andy reads books to children is mapped onto a semantic representation by the processing operations paraphrased in (1).

(1) Andy reads books to children.

a. Andy is identified as the first argument of the predicate read: $(a=$ Andy)

READ

$<$ a... $>$

b. books is identified as the second argument of read $(b=$ books)

READ

$<\mathrm{a}$ b...>

c. children is identified as the third argument of read ( $c=$ children)

READ

$<\mathrm{a} b \mathrm{c}>$ 


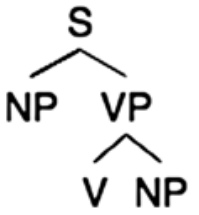

Figure 1 (O’Grady). Flat syntactic structure.

In this approach, priming is the product of processing: The activation of the three operations in (1) favors their reactivation, in the same order, at the next opportunity - the classic priming effect.

One way to tease apart the two views of priming is to focus on VP constituents, whose existence is posited by the flat-structure theory (see Fig. 1) but not by the direct-mapping theory, which eschews syntactic structure. A constituent-based account of priming such as B\&P's makes two predictions: (1) constituents (such as VP) should trigger priming effects; and (2) non-constituents should not. I will focus here on the latter prediction, with special attention to the subject-verb relation.

Because subjects and verbs do not form a constituent, B\&P's theory predicts an absence of priming effects for this relation. In contrast, the direct mapping view predicts that there could and should be priming effects of this type, because there is a processing operation that associates a clause's first argument with the verb (see [1a]). To my knowledge, there have been no specific tests of this claim, but several candidate phenomena come to mind, of which I will mention four here.

First, it is well known that certain items manifest variation in the type of agreement they trigger in the verb. Neither is one such word (for a discussion of a similar effect with certain collectives, such as couple, see Bock et al. 2007).

(2) a. Neither is satisfactory.

b. Neither are satisfactory.

If the choice of number agreement (is versus are) in these patterns could be primed by the choice made in a previous pattern with a similar type of subject, we would have prima facie evidence of priming by a subject-verb combination, contra the constituentbased theory.

A second candidate phenomenon occurs in languages that allow partial agreement with a coordinate NP subject. As illustrated in (3), verbs in Tsez can agree either with a full coordinate NP, yielding a plural form, or with just the nearest conjunct, giving a singular form. (Tsez is a Nakh-Dagestanian language spoken in the NE Caucasus; the data below are from Polinsky [2009]; ABS = absolutive, $\mathrm{PL}=$ plural, $\mathrm{PST}=$ past, $\mathbf{M}=$ masculine, $\mathbf{S G}=$ singular. $)$

(3) a. Full agreement with the subject:

kid-no uži-n b-ik'-s.

girl-ABS boy-AND PL-go-PST

The girl and the boy went.

b. Partial agreement with the subject's second conjunct:

kid-no uži-n $\boldsymbol{\emptyset}$-ik'-s.

girl-ABS boy-AND M,SG-go-PST

The girl and the boy went.

Here again, evidence that either pattern of subject-verb agreement triggers a priming effect would create a challenge for a constituent-based theory but not for a theory based on processing operations and direct mapping.

A third phenomenon of interest involves optional subject marking, which is especially common in ergative languages. The following example is from the Tibeto-Burman language Mongsen Ao (McGregor 2009, p. 496). (ERG = ergative, DET = determiner, $\mathrm{PRS}=$ present, $\mathrm{DECL}=$ declarative $)$

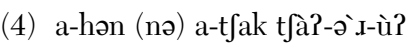

DET-chicken ERG DET-paddy eat-PRS-DECL

The chickens are eating paddy.
Because ergative case marks a relationship between a subject and a transitive verb, its primability would further challenge either the existence of VPs, or the viability of the view that priming faithfully reflects constituent structure, or both.

Finally, given possible independent restrictions on certain types of inflectionally triggered structural priming (Santesteban et al. 2015), it is important to consider subject-verb relationships that do not involve this phenomenon. The scopal ambiguity illustrated in (5) is a case in point.

\section{(5) Everyone didn't finish the project.}

This sentence permits two interpretations: Not everyone finished, with wide scope for the negative, and No one finished, with wide scope for the universal quantifier. Viau et al. (2010) showed that children's access to the first interpretation increases significantly when primed by exposure to similar sentences in contexts that support the not every reading. Although B\&P suggest that scopal relations are represented at a special level of semantic structure, the fact they can be primed by a subject-verb combination creates a potential problem for the constituent-based account.

Priming is important in its own right and as a window into the workings of language. In the best case, it may even provide insights into the nature of syntactic representations. Crucially, however, those insights may well point to a type of sentential architecture quite different both from the widely held view and from B\&P's proposal.

\section{Structural priming is a useful but imperfect technique for studying all linguistic representations, including those of pragmatics}

doi:10.1017/S0140525X17000553, e307

\author{
Alice Rees and Lewis Bott \\ School of Psychology, Cardiff University, Cardiff CF10 3AT, United Kingdom. \\ Reesa34@cardiff.ac.uk Bottla@cardiff.ac.uk \\ http://psych.cf.ac.uk/contactsandpeople/reesa34.php \\ http://psych.cf.ac.uk/contactsandpeople/bottla.php
}

Abstract: Structural priming is a useful tool for investigating linguistics representations. We argue that structural priming can be extended to the investigation of pragmatic representations such as Gricean enrichments. That is not to say priming is without its limitations, however. Interpreting a failure to observe priming may not be as simple as Branigan \& Pickering (B\&P) imply.

We agree with Branigan \& Pickering's (B\&P's) central thesis: Structural priming is a good methodological candidate for investigating linguistic representations. Structural priming, however, can be used to investigate representations used in pragmatics, as well as in semantics and syntax.

Bott and Chemla (2016) and Rees and Bott (2015; 2016) find that scalar implicatures - the prototypical pragmatic enrichment can be primed. For example, Bott and Chemla showed that sentences with enriched interpretations of some (some à some but not all) prime higher rates of enrichment in subsequent target sentences than sentences with basic some (where some takes its literal meaning; i.e., some and possibly all). These results suggest that another layer of representation could be added to Figure 1 in $\mathrm{B} \& \mathrm{P}$, with corresponding links to the lexicon. The representations involved would be at the sentence level ( $\left.\mathrm{S} \& \operatorname{not}\left[\mathrm{S}^{\prime}\right]\right)$, where $S$ refers to the basic, unenriched sentence, and $S$ ' to the informationally stronger sentence (a sentence involving a stronger expression, e.g., all in the case of some). Evidence that such a representation was independent of lexical material was given by the demonstration that sentences with implicatures associated with one expression, for example, some, could prime implicatures associated with another - for example, the numbers (from at least $N$ to 
exactly $N$ ), and evidence of links to the lexicon was shown by a lexical boost to the priming effect (some $\rightarrow$ some generated more priming than some $\rightarrow$ numbers). Finally, evidence that the priming effect was independent of the processes accessing the representations was provided by Rees and Bott (2016), who showed that production of implicatures could be primed by comprehension.

Why should these results demonstrate a separate level of representation, as distinct from the semantic level of B\&P? The distinction between semantics and pragmatics is fuzzy in the case of scalar implicatures (see so-called Grammatical Theories of scalar implicature, e.g., Chierchia [2013]). There are at least two differences between the representations described above and those included in Figure 1. The first is that representations used to generate scalar implicatures must take alternatives as part of their input - that is, sentences that the speaker could have said but didn't, for example, sentences involving all instead of some, as Grice (1975) and many others have argued. Correspondingly, Rees and Bott (2015) showed that sentences involving the alternative prime enrichment just as much as sentences involving the enriched scalar expression, and more than sentences involving the unenriched scalar expression. This type of input, and corresponding priming effects, do not apply to the semantic representations described by $\mathrm{B} \& \mathrm{P}$. The second difference is that implicature representations are applied optionally (or defeasible), as in the standard Gricean model, for example, in the case of comprehension, application of the implicature representation would be blocked if the speaker is not judged to have had sufficient knowledge to have uttered the stronger expression. The sorts of representations discussed by $\mathrm{B} \& \mathrm{P}$ are not optional in the same sense. Overall, then, while the use of structural priming so far has been used primarily to discover syntactic representations, it also can provide useful insight into how pragmatics can be integrated into a representational language system.

In the remainder of the commentary, we make two methodological comments on structural priming. The first is that structural priming provides causal information about similarities in representations, whereas many traditional linguistic techniques, such as analysis of corpora, provide only correlational information. Bott and Chemla (2016) illustrate this. They tested whether expressions that are uncontroversially enriched using Gricean mechanisms share derivation properties with other, more debatable implicature enriched meanings. They showed that enriched some primes enriched numbers but not enriched plural morphol$\operatorname{ogy}(+\mathrm{s})$. Consequently, they argued that some enrichment and the numbers shared a common derivation mechanism that was at least partially separate from the mechanism used to derive plural enrichment. Previous work addressing this question investigated the similarities in the enrichment distributions across expressions and context. For example, Horn (1972) observed that the numbers have an enriched meaning (exactly $N$ ) in the same contexts as some has an enriched meaning (some but not all), while Breheny (2008) and others found differences in the distributions. Distributional analyses, however, require considering examples in situ, complete with linguistic material that may or may not be relevant. Conversely, in structural priming, the potentially redundant material can be stripped away (or even investigated, as in the case of the lexical boost). The causal inferences that arise from structural priming make it a particularly powerful technique for discovering overlapping mechanisms and representations across linguistic phenomena.

The second point relates to the inferences that can be drawn in the absence of a priming effect. When two sentences that are hypothesized to use overlapping representations fail to prime each other, does this mean that the hypothesized representations are inaccessible (i.e., non-existent)? For example, if Bott and Chemla (2016) had failed to find that enriched some and numbers primed each other, would this constitute evidence that there are no abstract scalar implicature representations? B\&P do not directly address the issue, but they imply that representations are accessible only if they can be primed (sect. 1.4). While it is true that many representations are primable (i.e., they remain active across time), we do not feel that, to be accessible, representations necessarily must be primable. Primability confers many advantages, including the facilitation of alignment and prediction in dialogue (Pickering \& Garrod 2004; 2014). For some representations, however, these factors may not be important. Such representations would clearly not be detectible using structural priming methodology but might be accessible using other techniques. Therefore, a failure to observe a priming effect is an ambiguous result: Either the representation is not accessible, or it is not primeable. This is problematic, because a weakness in the alternative hypothesis makes null structural priming effects difficult to interpret and positive findings less persuasive (e.g., a "file drawer" effect is more likely when publication of a null effect is difficult).

\section{Developmental psycholinguistics teaches us that we need multi-method, not single-method, approaches to the study of linguistic representation}

\author{
doi:10.1017/S0140525X17000565, e308
}

Caroline F. Rowland ${ }^{\mathrm{a}}$ and Padraic Monaghan ${ }^{\mathrm{b}, \mathrm{c}}$

a'Language Development Department, Max Planck Institute for Psycholinguistics Max Planck Institute for Psycholinguistics, 6525 XD Nijmegen, Netherlands; ${ }^{\mathrm{b}}$ Psychology of Language Department, Max Planck Institute for Psycholinguistics Max Planck Institute for Psycholinguistics, 6525 XD Nijmegen, Netherlands; ' $E S R C$ LuCiD Centre, Department of Psychology, Lancaster University, Lancaster LA1 4YF, United Kingdom.

caroline.rowland@mpi.nl

http://www.mpi.nl/people/rowland-caroline

p.monaghan@lancaster.ac.uk

http://www.lancaster.ac.uk/staff/monaghan

Abstract: In developmental psycholinguistics, we have, for many years, been generating and testing theories that propose both descriptions of adult representations and explanations of how those representations develop. We have learnt that restricting ourselves to any one methodology yields only incomplete data about the nature of linguistic representations. We argue that we need a multi-method approach to the study of representation.

Branigan \& Pickering $(\mathrm{B} \& \mathrm{P})$ rightly state that acceptability judgments access linguistic representations only indirectly via language comprehension and production processes. This makes it difficult to draw strong conclusions about the nature of representations, because "the data are compatible with particular grammar-processor pairings, not just with particular grammars" (sect. 1.1, para. 4-5).

This problem, however, applies to all methodologies, including priming. In developmental psycholinguistics, we generate and test theories that propose both descriptions of adult representations and explanations of how those representations develop (e.g., Goldberg 2006; Pinker 1984). We have learnt that restricting ourselves to any method - even a well-studied method like priming yields only incomplete data about the nature of linguistic representation. For example, in priming studies, we access children's linguistic representation through the lens of a still poorly understood effect of priming on children's sentence production. To interpret our data, we must make inferences about the mechanisms underlying priming and how these mechanisms use the child's emerging linguistic knowledge. If our inferences about those processes (and how they use linguistic representations) are flawed, the conclusions we draw about representations will be flawed. In other words, if we rely on priming only, we will 
generate an incomplete theory of linguistic representations, which is likely to fail once tested using different methodologies.

The solution is a multi-method approach. As we have argued in Monaghan and Rowland (2017), by gathering evidence from different methods, we can converge on a more holistic understanding of the child's developing representations. Below, we illustrate our argument with two examples.

First, we examine how to determine the linguistic representations children hold at different ages. Structural priming studies have been informative here, showing that even young children's syntactic representations are abstract enough to support generalisation across verbs. For example, 3-year-old children produce more double-object datives (DODs) after a double-object dative prime than after a prepositional dative (PD) prime, even when the prime and target sentences share no content words (Peter et al. 2015; Rowland et al. 2012; see Thothathiri \& Snedeker (2008b) for similar results in comprehension).

It is tempting to conclude from this that children's dative representations are not only abstract, but also adultlike - that "evidence from these studies suggests that, from a relatively young age, children's structural representations are similar to adults" (sect. 3.3, para. 2). Although priming studies tell us that children's dative representations are abstract, however, they are not necessarily adultlike. This would be to assume that the priming mechanism requires adultlike representations, which is yet to be ascertained.

In fact, findings from other methodologies reveal asymmetries in the pattern of PD and DOD acquisition, which suggest that the two are not equally adultlike early on. Although naturalistic studies show that children produce DODs earlier than PDs (Snyder \& Stromswold 1997), early DOD use is restricted to a small set of high-frequency verbs (Campbell \& Tomasello 2001). Children are more productive earlier with the PD, in the sense of being more willing to use PD structures in novel verb experiments. For example, Conwell \& Demuth (2007) showed that 3-year-olds were more likely to generalise a novel verb heard in a DOD to a prepositional form (e.g., to produce he pilked the cup to Toby after hearing I pilked Toby the cup) than they were to generalise a novel verb heard in a PD to a doubleobject form. There is a similar asymmetry in novel verb comprehension (Rowland \& Noble 2011).

A number of explanations might integrate these findings. Perhaps children's very early double-object datives are restricted to a few, frequent verb-specific patterns, which become so entrenched that it remains difficult to generalise the structure to novel verbs, even when representations become more abstract (Tomasello 2000). Alternatively, PD representations may have a "head start on the process of becoming abstract" because of their structural similarity to the early acquired transitive structure (Campbell \& Tomasello 2001, p. 266). More work is needed here. Our point is simply that, without a multi-method approach, we would not gain these insights into the nature of children's developing knowledge.

Our second example demonstrates how a multi-method approach combining computational modelling with experimental work enables us to test the complex interplay between representation and the processing of those representations. Distinguishing processing from representation is far from trivial, as defenders of acceptability judgments have indicated (sect. 1.2, paras. 1-2). Consequently, a theoretical model, as presented by B\&P, provides only a first step as a description of representational features and the likely processes that operate over these representations. Computational modelling of experimental findings is needed to test the necessity and sufficiency of representation and processing in the language system, as well as the extent to which there is separability between representations and the processes operating over them.

Chang et al. (2006) showed that a computational model with distinct event semantics and syntactic knowledge was able to simulate a series of syntactic priming effects, but only when points of interaction between those representations was limited. Language, however, is acquired and processed in a rich, multimodal situation that goes far beyond the representations described in Chang et al. (2006). For instance, Smith et al. (2017b) constructed a computational model of processing in visual-world paradigm tasks, determining how phonological, visual, and semantic representations are integrated during speech perception. They demonstrated that the behavioural data could be simulated most effectively only when these representations interpenetrated throughout processing, rather than assuming autonomy of these representations, cohering at the outcome of processing. An extension of this model to language development showed that differences in quantity of exposure to a rich, multimodal language environment was sufficient to simulate child and adult behavioural differences in visual world paradigm tasks (Smith et al. 2017a). In other words, combining insights from the rich interactivity of multimodal information with the possible advantages of modular processing of this richness requires computational implementations to distinguish alternative accounts.

In sum, the process of determining linguistic representations from empirical data is far from straightforward and requires a multi-methodological approach.

\section{The malleability of linguistic representations poses a challenge to the priming-based experimental approach}

doi:10.1017/S0140525X17000577, e309

\author{
Rachel Ryskin and Sarah Brown-Schmidt ${ }^{\mathrm{b}}$ \\ ${ }^{a}$ Department of Brain and Cognitive Sciences, Massachusetts Institute of \\ Technology, Cambridge, MA 02139; ${ }^{\mathrm{b}}$ Department of Psychology and Human \\ Development, Vanderbilt University, Nashville, TN 37203. \\ ryskin@mit.edu sarahbrownschmidt@gmail.com
}

\begin{abstract}
Recent findings show that experience with a syntactic structure has long-term consequences for how that structure will be processed in the future, which suggests that linguistic representations are not static entities that can be probed reliably without alteration. Thus, leveraging the effect of previous exposure to a syntactic structure appears to be an inappropriate method for studying invariant properties of language.
\end{abstract}

We agree with Branigan \& Pickering $(\mathrm{B} \& \mathrm{P})$ that understanding the nature of linguistic representations is a key question for psycholinguists and linguists alike, but relying on acceptability judgments imposes serious limitations on what can be learned. Further, we agree that implicit processing measures can provide valuable insights about linguistic representations. We have concerns, however, about the use of syntactic priming as the primary measure for the investigation of purportedly stable linguistic representations. The first concern is of a practical nature: Syntactic priming effects are often subtle, and many of the potential links between structures of interest may be very difficult to detect reliably, rendering the picture more uncertain rather than clearer. Second, and more important, recent evidence suggests that language representations are not stable but rather continuously updated based on recent experience. The current proposal for a unified model of language representations, as seen through the lens of syntactic priming, can provide only a partial and possibly inaccurate account if it ignores the multiple timescales and contexts in which learning, and thus, language change, takes place.

The logic of the proposed method as described in the target article requires that (1) the facilitatory effect of a prime structure on a test structure be a direct reflection of how closely related those structures are in representational space; and (2) that the underlying representations of the language not be affected by recent exposure to either of the structures or their prior co-occurrence. In order for a syntactic priming paradigm to be a useful tool for understanding the structure of linguistic representation, it must be assumed that the act of processing the prime and target 
structures has no effect on that same language user's syntactic representations, or that such effects are transient (Branigan et al. 1999).

In contrast, recent findings show that experience with a syntactic structure has long-term consequences for how that structure will be processed in the future, both in comprehension (e.g., Fine et al. 2013; Fine \& Jaeger 2016; Kamide 2012; Long \& Prat 2008; Luka \& Barsalou 2005; Ryskin et al. 2017; Tooley et al. 2014b; Wells et al. 2009) and production (e.g., Bock et al. 2007; Bock \& Griffin 2000; Branigan et al. 2000; Hartsuiker et al. 2008; Jaeger \& Snider 2013; Kaschak et al. 2014; 2011a; 2011b). These results point to an error-based learning mechanism underlying syntactic priming across modalities (Chang et al. 2006; Chang et al. 2012). On this account, exposure to the prime sentence makes a sentence with that same structure more expected, and this up-weighting process is cumulative, such that the target sentence also serves the same function. Further, by the same error-based learning mechanism, the co-occurrence of structures with particular lexical items in the sentences should lead to an increased expectation for that combination in the future. Indeed, repeatedly exposing listeners to a novel pairing of a verb and a syntactic structure- $-\underline{R u b}$ the duck with the lollipop which is high attachment (the ambiguous $\mathrm{PP}$, with the lollipop attaches to the verb) if the visual context constrains the lollipop to be an instrument, but low attachment (the ambiguous PP attaches to the head noun) if the lollipop is an accessory tied to the duck-leads listeners to form a new verb bias (Ryskin et al. 2017). In other words, the representation of a verb and its link to a structure is malleable and shaped by the context in which that verb appears. This context may consist of the other words in the sentence, the visual environment, or even the identity of the speaker (Kamide 2012).

Does this present a challenge for the use of syntactic priming as a tool for studying language representations? Evidence for the cumulative learning underlying syntactic representations suggests that syntactic priming experiments often may miss the links investigators wish to uncover. In a paradigm where test stimulus $\mathrm{A}$ is preceded by primes $\mathrm{B}$ or $\mathrm{C}$, the presentations of $\mathrm{A}, \mathrm{B}$, and $\mathrm{C}$ lead to those representations becoming more active or more probable. Given that priming experiments typically involve repeated measures, over the course of the task the activation level for all relevant structures may be brought to some ceiling level at which the additional facilitation conferred by $\mathrm{B}$ is no longer detectable. Indeed, cumulative priming may contribute to the small effect sizes observed in the priming literature (Mahowald et al. 2016b; Tooley \& Traxler 2010; see Fine et al. 2013 for a similar methodological point). Continued use of syntactic priming to test more and more subtle connections between structures will likely lead to many null findings from which no conclusions about the true nature of syntactic representations can be drawn.

From a theory-building perspective, the proposed approach overlooks a large swath of the current literature and thus can provide only an incomplete picture of language representation. On many current accounts (Chang et al. 2006; Chang et al. 2012; Jaeger \& Snider 2013; Fine et al. 2013), syntactic priming effects are a byproduct of the continuous learning that shapes language representations. Based on these findings, we can infer that experiences with individual prime or target structures will accumulate to change the relative probabilities of these structures. Further, the probabilistic bindings between lexical items (e.g., verbs) and structures are subject to the same cumulative learning through verb-structure co-activation. In this context, the question of whether structure A is more similar to B or C may be ill posed. The answer provided by a syntactic priming experiment may be entirely dependent on the particular time-point at which the language user is probed and what linguistic experiences preceded this sampling. To understand the nature of linguistic representations, then, a more useful approach will be to examine how the learning prompted by interpretation of a single utterance in a given context, scales up to support language learning and language change throughout the lifespan.

\section{The relationship between priming and linguistic representations is mediated by processing constraints}

\author{
doi:10.1017/S0140525X17000589, e310
}

\section{Robert Slevc ${ }^{\mathrm{a}}$ and Iva Ivanova ${ }^{\mathrm{b}}$ \\ ${ }^{a}$ Department of Psychology, University of Maryland, College Park, MD 20742; ${ }^{\mathrm{b}}$ Department of Psychology, University of Texas at EI Paso, El Paso, TX 79902. slevc@umd.edu http://Imcl.umd.edu imivanova@utep.edu https://academics.utep.edu/Default.aspx?tabid=76103}

Abstract: Understanding the nature of linguistic representations undoubtedly will benefit from multiple types of evidence, including structural priming. Here, we argue that successfully gaining linguistic insights from structural priming requires us to better understand (1) the precise mappings between linguistic input and comprehenders' syntactic knowledge; and (2) the role of cognitive faculties such as memory and attention in structural priming.

Branigan \& Pickering $(\mathrm{B} \& \mathrm{P})$ provide a strong argument that structural priming (and priming paradigms more generally) may provide a useful avenue to better understand the nature of linguistic representations. We wholeheartedly agree that structural priming can yield useful insights about individuals' underlying linguistic representations. However, just as insights from metalinguistic acceptability judgments require us to understand how these judgments are made, insights from priming paradigms require us to understand the processes underlying structural priming - specifically, how priming can be influenced by online processing and cognitive constraints.

To understand structural priming, we first need to understand the precise mappings between linguistic input (prime sentences) and comprehenders' syntactic knowledge. There is now considerable evidence that comprehenders' parses do not always reflect the input veridically. Instead, parses reflect comprehenders' recent experiences, prior beliefs, and predictions of upcoming linguistic material (review: Traxler 2014). Critically, these factors not only influence sentence interpretation, but also influence syntactic parses themselves. An important question, then, is "What aspects of the parsing process influence patterns of structural priming?"

On one hand, there is evidence that structural priming reflects comprehenders' eventual parses, which may be the result of correction or reconstruction of the original linguistic input. For example, structural priming can occur from isolated verbs: Verbs that occur only, or mostly, in one construction can prime that construction (Melinger \& Dobel 2005). However, counterintuitively, structural priming also can occur from sentences with missing verbs (e.g., The waitress the book to the monk), and such priming has a similar magnitude to priming from well-formed primes (e.g., The waitress gives the book to the monk; Ivanova et al. 2017). These data suggest that comprehenders can reconstruct missing verb categories as well as missing post-verbal syntactic constituents, and these reconstructed representations give rise to priming effects. Structural priming even can reflect apparent corrections of parses that would result in implausible scenarios. That is, after implausible double-object datives such as The waitress gave the book the monk, speakers can be primed to produce prepositional datives and not double objects as might be expected based on a syntactic analysis alone (Slevc \& Momma 2015). These data suggest that non-syntactic factors such as plausibility can influence a comprehender's final parse, thus changing or even reversing expected patterns of structural priming. Crucially, in all three examples, priming effects appear to reflect listeners' final parses rather than the structure of the original input.

On the other hand, structural priming can reflect intermediate aspects of parsing, such as erroneous abandoned partial analyses, instead of, or in addition to, the intended and presumably eventual parse. For example, an incomplete sentence fragment that is subsequently corrected to another syntactic structure still can produce structural priming. That is, speakers were more likely 
to describe a picture with a prepositional dative after hearing a sentence fragment that started as a prepositional dative but was corrected to a transitive (e.g., The mechanic is giving the new part... uh... is recognizing the new part) than after a fragment starting as a double-object dative and corrected to a transitive (Slevc \& Ferreira 2013). Similarly, participants completed more sentences as transitives after temporarily ambiguous sentences such as While the man was visiting the children who were surprisingly pleasant and funny played outside than after identical sentences disambiguated by a comma (van Gompel et al. 2006). Importantly, the priming effects observed in these studies reflected temporarily suboptimal or erroneous parses that arose from the processing demands of online parsing.

Of course, structural priming reflects active processing even in the absence of errors or temporary ambiguity. Thus, the role of underlying cognitive faculties is a second aspect of structural priming that deserves more investigation. One such faculty is attention: Priming effects are greater when primes are directly attended (e.g., when a comprehender is addressed directly in a dialogue) compared to when she or he is not directly addressed (e.g., when a comprehender simply overhears a conversation). This result suggests that the depth of processing of a prime sentence directly affects the magnitude of priming (Branigan et al. 2007). A second relevant faculty is working memory. Although there is evidence that structural priming effects can be long lasting (e.g., Bock \& Griffin 2000; Kaschak et al. 2011b) and may reflect implicit learning rather than short-term maintenance (e.g., Chang et al. 2006), working memory nonetheless has been implicated in structural priming. Ivanova et al. (2013) found that increased working memory demands during the production of target picture descriptions reduced priming (at least for datives in a picture description paradigm; note that priming the presence/absence of the complementizer that in a recall paradigm was unaffected by memory load). These data suggest that attention and memory demands can influence priming effects, although we still know very little about these influences.

These examples illustrate that the relationships among linguistic input, syntactic knowledge, and structural priming are indirect and mediated by processing constraints. This does not undermine the usefulness of structural priming to shed light on linguistic representations. It does suggest, however, that more work is needed to understand how specific task and stimuli details affect both parsing and structural priming. Of course, processing dynamics influence metalinguistic acceptability judgments as well (e.g., Lau \& Ferreira 2005), and so it will be important to compare how processing demands affect these different paradigms. More generally, structural priming (like any method) has both advantages and limitations. We agree with Branigan and Pickering that it can be a useful tool to investigate the nature of linguistic representations, but we also caution that this tool still requires careful work to unpack the processes underlying our tendency to reuse recently experienced structure.

\section{Setting the empirical record straight: Acceptability judgments appear to be reliable, robust, and replicable}

\section{doi:10.1017/S0140525X17000590, e311}

\section{Jon Sprouse ${ }^{\mathrm{a}}$ and Diogo Almeida ${ }^{\mathrm{b}}$ \\ ${ }^{a}$ Department of Linguistics, University of Connecticut, Storrs, CT 06269-1145; ${ }^{\mathrm{b} D e p a r t m e n t ~ o f ~ P s y c h o l o g y, ~ N e w ~ Y o r k ~ U n i v e r s i t y ~ A b u ~ D h a b i, ~ A b u ~ D h a b i, ~}$ United Arab Emirates. \\ jon.sprouse@uconn.edu www.sprouse.uconn.edu \\ diogo@nyu.edu \\ http://nyuad.nyu.edu/en/academics/faculty/diogo-almeida.html}

Abstract: Branigan \& Pickering (B\&P) advocate the use of syntactic priming to investigate linguistic representations and argue that it overcomes several purported deficiencies of acceptability judgments. While we recognize the merit of drawing attention to a potentially underexplored experimental methodology in language science, we do not believe that the empirical evidence supports B\&P's claims about acceptability judgments. We present the relevant evidence.

Branigan \& Pickering $(\mathrm{B} \& \mathrm{P})$ advocate the use of syntactic priming to investigate linguistic representations. We support the use of any data types that scientists find relevant for specific research questions, including syntactic priming. We regret, then, that $\mathrm{B} \& \mathrm{P}$ appear to repeat unsubstantiated claims that paint a relatively misleading picture of acceptability judgments (AJs), a data type that linguists have been using fruitfully for decades. From our perspective, much of the literature criticizing AJs has repeatedly focused on logically possible concerns about their use without investigating whether those concerns are empirically attested. This risks a vicious circle: Articles can cite each other for support, giving the illusion of empirical support. In this commentary, we highlight a number of studies that have pursued this issue head on, which we leverage to examine six of B\&P's claims about AJs in detail.

Claim 1: Linguists standardly ask a single informant about the acceptability of a few sentences (sect. 1.2, para. 2). Claim 1 is a caricature of linguistic methodology that, to our knowledge, has never been supported by evidence. Nonetheless, a charitable interpretation of this claim reveals two separate concerns: (1) the routine use of small sample sizes, and (2) the susceptibility of AJs to investigator bias (Claim 2, below). An obvious consequence of using small samples sizes in research is an increase in errors (probably of all four types identified by Gelman \& Carlin 2014: I, II, Sign, and Magnitude). By performing a large-scale comparison of the published results in linguistics with retests of those results using large samples of naïve participants, one can evaluate the quality of their convergence rate. This cannot identify specific errors, but it can tell us whether the differences between methods actually produce different results.

Sprouse and Almeida (2012) tested every English data point from a popular syntax textbook (Adger 2003) using large samples of naïve participants. Out of 365 phenomena, they conservatively estimate a minimum convergence rate of $98 \%$. Sprouse et al. (2013) randomly sampled 148 phenomena from a leading linguistics journal (Linguistic Inquiry), and conservatively estimate a convergence rate of $95 \%( \pm 5 \%$ because of the random sampling). These high (conservative) convergence rates suggest that the sample sizes used by linguists (whatever they are) historically have introduced little error to the empirical record for any combination of the following reasons: (1) the samples are larger than what critics claim; (2) the effect sizes are so large that small samples still yield good statistical power; or (3) AJ results are highly replicated before and after publication (e.g., Phillips 2009).

Claim 2: Acceptability judgments are highly susceptible to theoretical cognitive bias because linguists tend to use professional linguists as participants (sect. 1.2, para. 3). This can also be addressed by the studies discussed above. Cognitive bias should predict sign reversals between naïve and expert populations. Sprouse and Almeida (2012) found no sign reversals from the textbook data. Sprouse et al. (2013) reported a 1-3\% sign reversal rate in the journal data. Mahowald et al. (2016a) and Häussler et al. (2016) have replicated the latter without reporting an increased sign reversal rate $(0-6 \%)$. Comparisons of naïve and expert populations also were conducted by Culbertson and Gross (2009), who report high inter- and intra-group correlations on 73 sentence types, and by Dacbrowska (2010). The latter found that, while experts gave less variable ratings than naïve participants on several sentence types, experts rated certain theoretically interesting syntactic violations as more acceptable than naïve participants, in apparent conflict with their theoretical commitments. Taken together, these results are not what one would expect if AJs were highly susceptible to cognitive bias. 
Claim 3: Acceptability judgments are susceptible to differences in instructions (sect. 1.2, para. 3). Claim 3 has been directly investigated by Cowart (1997), who reports that the systematic manipulation of instructions does not change the pattern of acceptability judgments for factorial designs.

Claim 4: Acceptability judgments are impacted by sentence processing effects (sect. 1.2, para. 5). Claim 4 is technically true, but $\mathrm{B} \& \mathrm{P}$ exaggerate its consequences. First, many classic lexical and sentence processing effects have relatively small or negligible effects on acceptability (e.g., Featherston 2009; Phillips 2009; Sprouse 2008; Sprouse et al. 2012). Second, very few syntactic phenomena have been proposed to be fully reducible to sentence processing effects. The lone exceptions to this appear to be constraints on long-distance dependencies (e.g., Kluender \& Kutas 1993; Hofmeister \& Sag 2010), but in that case, a number of experimental studies have disproven the reductionist predictions (Phillips 2006; Sprouse et al. 2012; Yoshida et al. 2014). Thus, to the extent that AJs are impacted by sentence processing, it appears as though the effects can be dealt with like any other source of noise in an experimental setting.

Claim 5: Acceptability judgments reveal only set membership (sect. 1.2, para. 7). Claim 5 is confusing. It is false in the sense that, if one is interested in set membership, this property still needs to be inferred from acceptability data, using a logic that maps that data type back to the relevant cognitive computations. In this, AJs are like any other data type in cognitive science: No data types, including priming, directly reveal the underlying computations of the human brain, and all data types require a linking hypothesis between the observable data and the unobservable cognitive process.

Claim 6: Acceptability judgments have yielded no consensus theory among linguists (sect. 1.2, para. 9). Claim 6 is a strange criticism to make of any data type, especially AJs. First, the beliefs of scientists are a subjective issue based on how they weigh different kinds of evidence. Second, AJs are, by all accounts, a robust and replicable data type. Whatever disagreements there are in linguistics literature, they appear to obtain mostly at the level of interpreting, not establishing, the data (e.g., Phillips 2009).

In conclusion, we support B\&P's desire to bring new evidence to bear on questions about linguistic representation. We caution, however, that advocacy for one method should not be bolstered by misleading comparisons, especially with methods such as AJs, which yield data that are demonstrably robust, highly replicable, and comparatively convenient and inexpensive to collect.

\section{Priming is swell, but it's far from simple}

\section{doi:10.1017/S0140525X17000607, e312}

\section{Jayden Ziegler, ${ }^{a}$ Jesse Snedeker, ${ }^{a}$ and Eva Wittenberg ${ }^{b}$ aDepartment of Psychology, Harvard University, Cambridge, MA 02138; ${ }^{b}$ Department of Linguistics, University of California, La Jolla, CA 92093. ziegler@g.harvard.edu http://www.jaydenziegler.com snedeker@wjh.harvard.edu https://software.rc.fas.harvard.edu/lds/ research/snedeker/jesse-snedeker/ ewittenberg@ucsd.edu http://www.evawittenberg.com/i/start.html}

\begin{abstract}
Clearly, structural priming is a valuable tool for probing linguistic representation. But we don't think that the existing results provide strong support for Branigan \& Pickering's (B\&P's) model, largely because the priming effects are more confusing and diverse than their theory would suggest. Fortunately, there are a number of other experimental tools available, and linguists are increasingly making use of them.
\end{abstract}

Branigan \& Pickering (B\&P) tell a straightforward tale. Linguists rely on grammaticality judgments to uncover representations.
Judgments have limitations, but no other psycholinguistic methods systematically reveal linguistic structure. Fortunately, priming offers a direct window onto representation, providing evidence for two distinct levels: a surface syntactic form, independent of meaning and void of lexical content, and a semantic form that includes information about thematic roles, quantifier scope, and information structure.

We are fond of priming ourselves, but this elegant story is misleading in several ways. First, the priming literature does not strongly support the theory that B\&P propose. As they dive deeper, the loose ends and contradictions emerge, but their final conclusions bypass this complexity. If we rearrange the evidence a bit, the theoretical ambiguity becomes clearer.

The primary evidence for syntactic representations comes from studies of argument alternations (dative or active-passive) that perfectly confound surface syntax with thematic mappings. $\mathrm{B} \& \mathrm{P}$ note that a few foundational studies demonstrated that syntax can be primed independent of thematic mappings (sect. 2.1). Thus, they privilege syntax in their theory. But there is now an equally robust literature demonstrating that thematic mappings can be primed independent of syntax (e.g., Cai et al. 2012; Chang et al. 2003; Cho-Reyes et al. 2016; Hare \& Goldberg 1999; Salamoura \& Williams 2007; Ziegler \& Snedeker 2016b). B\&P acknowledge this work (sect. 2.4) but treat it as a secondary, interface phenomenon: Thematic information remains separate from syntax (Fig. 1).

Similarly, the observation that priming can occur in the absence of lexical overlap motivates a theory in which the syntactic skeleton is separate from the lexical content. To account for the lexical boost, $\mathrm{B} \& \mathrm{P}$ must complicate their story, by linking lemmas to structures (sect. 2.3). But perhaps we should revisit the claim that the syntactic structure lacks lexical nodes. Indeed, function words can be a locus of priming (Bencini et al. 2002; Ferreira 2003). We know that only partial overlap in the syntactic skeleton is needed for structural priming (sect. 2.1), but we don't assume that the unnecessary pieces are removed from the syntactic representation. Lexical content may be similar: always present and sometimes contributing to priming via overlap.

The evidence for their semantic level is also sparse. We know: (1) Quantifier scope can be primed, (2) this priming is isolated to the particular quantifier used (e.g., each does not prime every), and (3) it abstracts away from the nouns and verbs in a sentence (Feiman \& Snedeker 2016; Raffray \& Pickering 2010). However, B\&P's claim that scopal priming is bound to thematic roles and cannot be captured by an LF representation is controversial (Chemla \& Bott 2015). It rests on a single null result with prime stimuli (A boy climbed every tree) that have not been shown to produce priming when thematic roles are the same. Furthermore, the manipulation used confounds verb-specific roles, thematic roles, and the notion of deep subject/object. It's just too early to conclude that scope and thematic roles are tightly coupled, or that LF isn't the locus of scopal priming.

It seems that, under the right conditions, almost any linguistic representation, mapping, or process can be primed. Consequently, evidence for priming is always interpretable to some degree (it demonstrates a commonality between prime and target). But the absence (or magnitude) of an effect is often less constraining, because there is so much variability across tasks and stimuli. In some comprehension tasks, there is no priming in the absence of verb overlap (Arai et al. 2007), while in others, abstract priming is robust (Thothathiri \& Snedeker 2008a; 2008b). This problem isn't unique to comprehension. The pattern of effects in production can depend on how the sentences are elicited (stem completion vs. full sentence generation; Ziegler \& Snedeker 2016a).

Understanding this instability is critical; we suspect that the answer lies in thinking through the processes involved in each task and how they engage both stored representations and representations that are constructed on the fly. To do this, we will have to move beyond the notion of priming as a static, atemporal 
phenomenon that targets stable representations independent of the process of production (or comprehension).

While priming is not the transparent window that $\mathrm{B} \& \mathrm{P}$ promise, psycholinguists do have a much wider range of tools than the paper suggests. Some are behavioral. Novel word generalization studies going back to the 1950s have revealed structural regularities in the representation of linguistic form and meaning (Berko 1958; Fisher 2002; Gropen et al. 1989; Naigles 1990; Prasada \& Pinker 1993). Similarly, artificial language learning sheds light on the representations that learners extract from linguistic data and use to guide generalization (Pothos 2007; Reber 1967; Saffran et al. 2008; for review, see Erickson \& Thiessen 2015).

New methods for analyzing imaging data also provide greater constraint on representational theories. For example, multivoxel pattern analysis, a class of machine-learning algorithms that examine patterns of neural activity (Haxby et al. 2001), has revealed regions of the left temporal cortex that appear to bind arguments to something roughly like thematic roles (Frankland \& Greene 2015). Structural priming is therefore only one useful tool of many.

Finally, we think that B\&P are too pessimistic in their assessment of linguists and their tools. There are longstanding traditions of experimental work in phonetics, phonology, and language acquisition. In recent years, experimental work has also become common in syntax, semantics, and pragmatics (e.g., Arunachalam 2013; Cowart 1997; Myers 2009; Sorace \& Keller 2005). Indeed, the question of how armchair judgments translate into generalizable conclusions has received considerable attention (Sprouse \& Almeida 2012; Sprouse et al. 2013). From our perspective, the remaining disputes do not reflect an over-reliance on grammaticality judgments or a dearth of appropriate methodologies; they stem from: (1) the close parallels between the theories that are still standing (similar operations assigned to different theoretical levels), (2) the lack of falsifiability for the contrasting features, and (3) the danger we all face of letting our "affection for [our] intellectual child[ren]" (Chamberlin 1897) guide our interpretation of the data.

\section{Authors’ Response}

\section{Structural priming and the representation of language}

\author{
doi:10.1017/S0140525X17001212, e313
}

\section{Holly P. Branigan and Martin J. Pickering \\ Department of Psychology, University of Edinburgh, Edinburgh, EH8 9JZ, United Kingdom. \\ holly.branigan@ed.ac.uk http://www.ed.ac.uk/profile/holly-branigan martin.pickering@ed.ac.uk \\ http://www.ed.ac.uk/profile/martin-pickering}

\begin{abstract}
Structural priming offers a powerful method for experimentally investigating the mental representation of linguistic structure. We clarify the nature of our proposal, justify the versatility of priming, consider alternative approaches, and discuss how our specific account can be extended to new questions as part of an interdisciplinary programme integrating linguistics and psychology as part of the cognitive sciences of language.
\end{abstract}

In our target article, we argue for an experimental approach to linguistic representation and demonstrate how structural priming can be used to develop a psychologically motivated account of how people represent linguistic structure. If one utterance primes another, then we argue that they share structure. Patterns of priming are therefore informative about the way in which people represent language. While acknowledging the continuing value and importance of acceptability judgements in developing representational hypotheses, we proposed that priming is in many ways superior: It is an implicit behavioral measure that taps representation. It also allows us to study representation in all groups, including those such as young children who cannot make acceptability judgments.

In recent years, many experimental studies using structural priming have helped us understand language users' representations. These studies suggest that semantic information is represented separately from syntactic information. The single semantic level encodes information about scope relations, information structure, and thematic structure. The single syntactic level, which draws on well-formedness constraints concerning local linear and hierarchical relations, includes syntactic category information and some missing elements (i.e., elements that are not uttered) but does not involve syntactic movement. At present, linguists propose incompatible theories, and there is no sign that the nearly exclusive use of acceptability judgments is ever going to determine which type of theory is correct. In contrast, the findings from priming are compatible with some theories and incompatible with others.

The commentators take a unified view that researchers from different disciplines should cooperate in investigating linguistic representation, as a single integrated programme of research. They unanimously agree that an experimental approach to linguistic representation is valuable and largely accept that structural priming is informative in this regard. In other words, they do not feel that it is relevant only to the study of how language is produced and comprehended. They differ, however, about the versatility of priming, how directly it taps into representation, and its advantages over other methods (in particular, acceptability judgments). They also take different views about our conclusions regarding aspects of linguistic representation and make specific proposals for further research.

We are pleased that our proposal has met with such interest and hope that it will encourage a future programme of cooperative interdisciplinary research on linguistic representation. In what follows, we have grouped our responses to their insightful comments under 11 headings.

\section{R1. How can linguistic representation be investigated?}

We begin by clarifying the nature of our proposal. Although commentators agree about the importance of experimental methods for investigating linguistic representation, some of them seem to assume that we advocate entirely renouncing the use of acceptability judgments. But as we make clear throughout the article, our argument is that researchers interested in linguistic representation should not be restricted to using only acceptability judgments. Specifically, we contend that "the representations underlying language use need not and, in fact, should not be investigated only via [acceptability] judgments" (sect. 1, para. 2). We further argue that "[our] goal is to consider alternative (experimental) methods to acceptability judgments that potentially address the linguistic representations implicated in language processing" (sect. 1.1, para. 5), that "acceptability judgments are not enough," (sect. 1.2) and that "researchers concerned with linguistic representations should not rely solely on such judgments, and should call on additional methodologies" (sect. 1.2, para. 10). Moreover, we argue that "acceptability judgments can be used (with appropriate controls) alongside structural priming (and perhaps other experimental methods; see sect. 1.3) as a means 
of developing representational hypotheses" (sect. 1.5, para. 2). (Emphasis added throughout.)

We therefore do not propose abandoning acceptability judgments as a linguistic method (Adger; de Ruiter \& de Ruiter; Hall, Mayberry, \& Ferreira [Hall et al.]), nor do we argue that structural priming is the only tool that can be used to study linguistic representation (González-Márquez, Feist, \& Ströbel [González-Márquez et al.]; Hartsuiker \& Bernolet; Ziegler, Snedeker, \& Wittenberg [Ziegler et al.]). (We suspect that some commentators may have misinterpreted the phrase "end the current reliance on acceptability judgments" in the abstract as an exhortation to "end the current use of acceptability judgments.")

We do argue, however, that priming has a privileged status. It is an implicit method that provides access to linguistic structure (i.e., strong generative capacity), and it is unaffected by an intervening theory "P" (Chomsky 1981, p.283, footnote 39); see section 1.1, paragraph 7 of the target article. Acceptability judgments (whether obtained informally or formally) provide extensive and valuable, but suggestive, data (in the "context of discovery"; Hagoort). In this respect, they stand alongside the use of large-scale corpora to identify the types of utterances that occur and the contexts in which they occur (as Lester, Du Bois, Gries, \& Martin [Lester et al.] note). Both of these methods provide evidence about the classes of acceptable sentences but need further assumptions to help determine linguistic structure - and here is where they encounter the problem of constituency tests. Other behavioral and neuroscientific methods (i.e., apart from priming) may provide evidence about linguistic structure without the need for constituency tests, but it is much harder to rule out explanations relating to the processing theory "P."

Adger argues that linguists use experimental tests of a theory "as an injunction to think about what the implications of the result mean." We agree that they should do this, but we do not believe that they typically do so (or else, papers in theoretical linguistics would make far more reference to experimental findings than they do). In sum, we wholeheartedly agree with Hagoort that there is no excuse for failing to use the available experimental and quantitative methods.

\section{R2. How useful is priming?}

A number of commentators express concerns about the versatility of structure priming in practice and the extent to which structural priming studies are able to provide support for specific representational proposals (de Ruiter \& de Ruiter; Feldman \& Milin; Francis; Hall et al.; Hartsuiker \& Bernolet; MacDonald \& Weiss; Mahowald, Futrell, \& Gibson [Mahowald et al.]; Maldonado, Spector, \& Chemla [Maldonado et al.]; Martin, Huettig, \& Nieuwland [Martin et al.]; Meyer; Rees \& Bott; Ziegler et al.).

As Mahowald et al. note, the meta-analysis carried out by Mahowald et al. (2016b) shows that structural priming is robust and highly replicable. Therefore, criticisms of other priming paradigms (e.g., in social psychology) are not relevant (MacDonald \& Weiss). The meta-analysis also highlights limitations in studies that compare the magnitude of priming under different circumstances. We stress that a large number of converging findings may be necessary to produce confidence in theoretical claims.

With respect to our specific account, our claims concerning the monostratal nature of syntactic representation are based on many individual studies that, together, argue against some level of underlying structure. Our intention in proposing the account in section 2 is to encourage researchers to address both specific and general representational questions, in order to broaden the evidence base for theory development. Some of our specific claims, of course, may prove incorrect or incomplete in light of further research.
Hence, we acknowledge a need for reliable, replicable results, and for caution about underpowered studies, the interpretation of null results, and so on (e.g., de Ruiter \& de Ruiter; Mahowald et al.; Maldonado et al.; Meyer; Rees \& Bott; Ziegler et al.). These concerns apply to any scientific method. Clearly, priming studies require careful design, and some studies may be difficult to carry out in practice. Structural priming, however, is not inherently more limited than other experimental (or nonexperimental) approaches.

Other commentators are concerned that the value of priming is limited to cases involving pairs of roughly equivalent structures (e.g., de Ruiter \& de Ruiter; Meyer). As we note, most current evidence comes from choices in language production, when a speaker can use one structure (e.g., passive) or another (e.g., active) to describe the same situation. In such cases, it is clearly necessary for alternatives to exist (and to be sufficiently frequent that, in practice, participants can be induced to produce both alternatives). But there are other methods of inducing priming, such as priming of alternatives that differ in meaning (Scheepers 2003), priming choices in comprehension (e.g., Branigan et al. 2005), priming of eye movements during comprehension (e.g., Arai et al. 2007), priming response times in production (Smith \& Wheeldon 2001; Segaert et al. 2016), priming response times in comprehension (Knoeferle 2014), or repetition suppression of the Blood Oxygen Level Dependent (BOLD) signal in functional Magnetic Resonance Imaging (fMRI) adaptation (Segaert et al. 2012). These methods do not always require semantically similar alternatives, and they should allow us to use priming more extensively in the future.

A particular set of priming effects might appear compatible with more than one linguistic account (e.g., Francis; Hartsuiker \& Bernolet). In some cases, there are ways to rule out one alternative (see sect. R10 for some specific examples). In other cases, the data may fail to distinguish between accounts - and this is why priming should be established as a general method in which conclusions are drawn from large numbers of studies considered together.

Some commentators raise concerns about the fact that priming effects reduce with increasing exposure (Lester et al.; Ryskin \& Brown-Schmidt). Experimenters can take this into account (especially when small effect sizes are anticipated) through careful decisions about the size and composition of the stimulus set, testing adequate number of participants and items, or appropriate statistical analyses. Ziegler et al. observe that magnitudes of priming may vary across tasks. This simply means that, just as for other experimental tasks (e.g., sentence recall versus picture description tasks), researchers should be careful about comparing results across tasks.

Finally, we do not share Meyer's pessimism concerning the relevance of priming for studying children and patients. For example, priming studies have successfully addressed longstanding questions about the extent to which typically developing children and children with a specific language impairment have abstract syntactic representations (Bencini \& Valian 2008; Foltz et al. 2015; Garraffa et al. 2015; Messenger et al. 2011; Rowland et al. 2012).

\section{R3. The directness of the relationship between grammar and processing}

MacDonald \& Weiss, Martin et al., and Meyer raise questions about the directness of the relationship between grammar and processing. Comprehension-to-production priming cannot be simply procedural because comprehension and production are different processes. Therefore, we suggest it provides access to representation (as argued in Branigan et al. 2000). Of course, it could reflect a shared sub-process - for example, accessing a linguistic constraint. But if this sub-process is the same across production and comprehension, it is hard to see what it could 
reflect except for the representation itself. In fact, any form of structural priming (including production-to-production) is informative about linguistic representation, and researchers do not need to conduct comprehension-to-production priming studies to constrain linguistic theory. Note that a linguistic theory could treat the frequency of a syntactic constraint (e.g., V $\rightarrow \mathrm{V} \mathrm{NP}$ $\mathrm{PP}$ ) as part of its linguistic representation (see MacDonald \& Weiss), but our concern is with the representation of the constraints themselves rather than their frequency.

We do not claim that the apparent automaticity of priming is necessary for making claims about representation rather than processes. Instead, it suggests that priming is not affected by strategies or metalinguistic processes-concerns that may affect acceptability judgments. In other words, apparent automaticity gives us more confidence about linguistic representations.

\section{R4. Does priming access all representations?}

Some commentators suggest that priming may be insensitive to some linguistic representations (Koring \& Reuland; Maldonado et al.; Rees \& Bott). In other words, there may be levels of linguistic representation that are unaffected by priming. Some representations may be inaccessible to any method, whether because they have been compiled into different representations online (Berwick \& Weinberg 1984) or because they have undergone attrition (see Kootstra \& Rossi). The commentators, however, are more concerned with the possibility that representations can be accessed but cannot be affected by prior exposure - a possibility that would reduce somewhat the effectiveness of priming. We propose that the use of a representation (whether in production or comprehension) will affect subsequent use of that representation; this is the logic that all accounts of priming depend on. But this is an assumption; if it is too strong, then priming would be somewhat less sensitive than we assume. Such a limitation, of course, would not affect the conclusions that we can draw from (positive) demonstrations of priming.

Of course, failure to find priming does not demonstrate that it does not occur; a study may have been insensitive or underpowered. For example, priming without lexical repetition in comprehension sometimes has occurred (e.g., Pickering et al. 2013; Thothathiri \& Snedeker (2008a)) and sometimes has not (e.g., Branigan et al. 2005; Traxler et al. 2014). Similarly, evidence for intonational priming is limited (de Ruiter \& de Ruiter), but to demonstrate that intonational structure is represented (in roughly the form assumed by many linguists) and that it cannot be primed would involve extensive experimentation. It is too early to conclude that there is a level of linguistic representation that cannot be primed.

\section{R5. What can acceptability judgments tell us?}

Adger; Ambridge; Gaston, Huang, \& Phillips [Gaston et al.]; Hall et al.; and Sprouse \& Almeida contend that we unfairly criticise a method that has been used fruitfully (and frugally) for decades-although other commentators (e.g., GonzálezMárquez et al.; Lester et al.) argue that we, in fact, overemphasize its significance. We acknowledge that acceptability judgments have been a fertile method for generating hypotheses historically, and we do not advocate abandoning them. Indeed, their use is appropriate for field linguistics (Hall et al.) when identifying the possible sentences of a language and developing representational hypotheses (i.e., in the "context of discovery"), but even in these situations, researchers should carry out priming experiments when possible.

Our main concern with acceptability judgments does not lie with their informality (Sprouse \& Almeida), though we do advocate avoiding informality where possible. We agree that careful experimentation can determine which judgments can be explained by processing limitations (e.g., Sprouse et al. 2012). Presumably, those judgments that cannot be explained by processing limitations are the judgments that are of interest to linguists, in the sense that they determine the set of sentences the grammar must be able to account for (i.e., its weak generative capacity). Sprouse and Almeida say that establishing these data is not in doubt, and the concern is with what they call interpreting them. In other words, they argue that determining the set of sentences generated by the grammar is straightforward (i.e., the weak generative capacity) and that the difficult issue is identifying the structural representations that underlie those sentences (i.e., the strong generative capacity).

There may not be major concerns in practice about identifying the set of sentences that should be generated by the linguistic theory (though there remain concerns about consistency across types of speaker and task; Dąbrowska 2010; Sprouse 2009). Acceptability judgments, however, do not allow us to determine the underlying structural representations, because they cannot distinguish among competing analyses of the same data. In contrast, priming allows us to distinguish among such analyses - in Sprouse and Almeida's terms, to interpret the data. People may use representations that intrinsically include lexical content, or they may not. They may use representations that include elements that are not phonologically realised, or they may not. The different representations proposed for DO sentences (e.g., a ternary-branching structure involving no "moved" elements versus a binary-branching structure involving "moved" elements; Pollard \& Sag 1994 versus Larson 1988) are different interpretations of the same data. But they cannot both correspond to a speaker's mental representation for these sentences.

Some commentators defend constituency tests. For example, Gaston et al. propose that the apparent contradictions that they yield can be resolved by further enquiry. This is not correct; their contradictions are basic and widespread. For example, almost anything can be coordinated, so it would appear that almost anything can be a constituent. But this claim is possible only if we assume massively flexible constituency (Pickering \& Barry 1993; Steedman 2000) - a proposal contested by the great majority of linguistic theories. And in practice, linguistic theories disagree about the most fundamental points regarding structure (not merely the structure of ellipsis or Wh-questions). Our point is that traditional linguistic approaches assume that strong generative capacity can be determined by combining the sets of sentences compatible with acceptability judgments (i.e., weak generative capacity) with constituency tests. But if the tests are as flawed as they appear to be, this is impossible, even if Sprouse and Almeida are correct about the consistency of acceptability judgments.

In response to the query of Gaston et al. about the nature of structural priming, the example of lexical priming that we use to introduce priming as a phenomenon (sect. 1.4) involves words that are related but not identical (i.e., associative/semantic priming). It may be more appropriate to compare structural priming with repetition priming of words (e.g., Scarborough et al. 1977), which is long lasting, like structural priming (Bock \& Griffin 2000). Structural priming appears to be relatively unaffected by contextual changes - for example, between writing and speech (Cleland \& Pickering 2006) - but a more detailed exploration of such issues is necessary to fully understanding priming itself. Understanding a method is, of course, very important for drawing conclusions using that method, but priming (as a cognitive phenomenon) is far better understood than the complex combination of language processing, metalinguistic reflection, and decision-making that underlies acceptability judgments. Similarly, there is no wellgrounded theoretical motivation for constituency tests (see sect. 1.2). 


\section{R6. The stability of linguistic representations}

Several commentators are concerned with the stability of the linguistic representations that are investigated in structural priming experiments (Cai \& Liu; Francis; Günther; Hall et al.; Kootstra \& Rossi; Martin et al.; MacDonald \& Weiss; Meyer; Ryskin \& Brown-Schmidt; Ziegler et al.). Some commentators suggest that their apparent instability and the fact that priming itself can bring about representational changes call into question the use of structural priming to investigate representation.

We agree that exposure to particular linguistic structures can affect speakers' linguistic representations, and any linguistic theory must be able to account for such effects. In practice, some researchers have argued that priming may offer an intervention to enhance foreign-language learning (McDonough \& Mackey 2006) or recovery from aphasia (Cho-Reyes \& Thompson 2012). Moreover, Ivanova et al. (2012b) found that participants occasionally produced a DO structure with a verb such as donate after encountering another DO sentence with donate, even though such sentences are usually regarded as unacceptable (Ambridge). In other words, priming can change the speaker's representation (at least temporarily). Other priming effects involve repeated exposure (e.g., developing the needs +ed construction; Kaschak \& Glenberg 2004). Importantly, similar effects occur in studies in which participants repeatedly make acceptability judgments, thereby showing that such judgments are affected also by exposure (Luka \& Barsalou 2005; Snyder 2000; cf. Sprouse 2009).

So, the method of investigation (priming) sometimes changes the phenomenon that it is investigating. But this observation is not problematic for our proposals. Our concern is with the nature of the representations that underlie language use at any given time, and such representations need not be static. Priming allows us to take a snapshot of linguistic representations. Moreover, we can track how those representations change across time and what causes them to change (e.g., Cai \& Liu; Kootstra \& Rossi; Rowland \& Monaghan). For example, structural priming supports a developmental trajectory during L2 learning from item-specific representations in less proficient L2 speakers to abstract representations in more proficient L2 speakers (Bernolet et al. 2013). Other studies have shown how children's syntactic representations at different ages do or do not differ from those of adults in specific ways - for example, with respect to their independence from lexical or semantic content (Messenger et al. $(2012 b))$. Of course, we do not claim that they are the same in all respects (Rowland \& Monaghan).

\section{R7. Priming, individuals, and groups}

Priming experiments are typically conducted over fairly large groups of participants, and some commentators (Hall et al.; MacDonald \& Weiss) therefore assume that anything priming tells us must be at the group level-that is, holding over groups of participants with the same linguistic representations as each other. We do not make this assumption. Researchers typically assume a common core of structural representations: that people who speak a particular language have similar linguistic representations. Of course, the acceptability judgment method also tends to make this assumption. But speakers of a language may have some different representations. Again, structural priming allows us to investigate variations across individual speakers (idiolectal variations) or populations (e.g., L1 versus L2 speakers of a language).

As with any other languages, priming should be used to understand the representation of sign languages (Hall et al.), and Hall et al. (2015) paves the way for such research. L1 and L2 ASL signers may have differences in syntactic processing, but the priming data suggest that - as with L1 and (proficient) L2 speakers of spoken languages (Schoonbaert et al. 2007)-the syntactic representations they use during processing may have the same (relevant) characteristics. In these studies, syntactic priming does not tell us how these representations were constructed during processing, their interfaces with other representations, how those representations are instantiated in the brain, or how they developed (although see Sleve \& Ivanova for relevant discussion).

Contrary to MacDonald \& Weiss's contention, it is possible to study priming in individual participants. Such studies might require extensive data from an individual, just as in any singlecase study. Moreover, if a structure can be represented variably within an individual, we can use priming to determine which representation is used more often (Günther).

Finally, Francis's observation that structural representations are not necessarily diachronically stable highlights how priming can address diachronic changes in structural representation (Pickering \& Garrod 2017). Her example shows how priming could distinguish between competing theoretical proposals that acceptability judgments have not been able to discriminate, in the same way as priming has been used to examine historically motivated accounts of lexical representation (Marslen-Wilson et al. 1994). In principle, corpus-based studies of structural priming (Gries 2005; Szmrecsanyi 2006) also could be used to track diachronic changes in structural representations.

\section{R8. Structural priming and language processing (in monologue and dialogue)}

A number of commentators discuss what priming may or may not tell us about language processing. Our focus in this paper is strictly on representation, so we consider processing only to the extent that it is informative about representation (thus, we do not address questions such as the timecourse of priming [Martin et al.], or how priming may change people's parsing preferences [Ryskin \& Brown-Schmidt]).

For example, Slevc \& Ivanova point out that priming can reflect not only people's final parses, but also their intermediate parses (e.g., Van Gompel et al. 2006). Therefore, we need to be careful about whether any priming effect depends on a temporary but abandoned representation rather than on the final representation. Investigations of abandoned analyses are, of course, also informative about the process of language comprehension (which analyses people consider, whether they hold on to abandoned analyses, and so on). Such issues, however, are not directly relevant to linguistic representation.

Sleve \& Ivanova also discuss how priming might interact with other aspects of cognition, such as depth of processing and working memory resources (see also Hartsuiker \& Bernolet; Martin et al.). These issues may be important to an understanding of processing - for example, with respect to the relationship between individual differences in priming and other aspects of cognition. Differences in method (and associated processing demands) also can affect priming magnitudes (e.g., Mahowald et al. 2016b; Ziegler et al.), so care must be taken when comparing studies. But if such factors are controlled within an experiment, they do not undermine the informativeness of priming for understanding linguistic representation, as Sleve \& Ivanova acknowledge.

We agree with Kootstra \& Rossi that naturalistic studies of priming are beneficial, but for current purposes (i.e., linguistic representation), it does not matter whether any effects occur under laboratory conditions or not. We are fascinated by the priming of code-switching, but this programme of research is relevant primarily to processing (i.e., language selection) - assuming, of course that the code-switcher selects between two independent representations of different languages. In sum, priming is versatile, and its implications go well beyond linguistic representation (see Pickering \& Ferreira 2008). 
MacDonald \& Weiss argue that priming may reflect a general (nonlinguistic) tendency to repeat prior actions, as demonstrated by parallel priming effects in motor actions and syntax (Allen et al. 2010) and between domains such as music and language (van der Cavey \& Hartsuiker 2016) (see also Kempson \& Gregoromichelaki). But if priming occurs between language and non-language, it does not prevent researchers using priming to discriminate linguistic theories; it is just that some representations are shared with other domains. To take a more formal example, Steedman (2002) presented an account of action based on principles (functional composition and type raising) that are used also in Combinatorial Categorial Grammar (Steedman 2000). Extending these principles to action in general does not invalidate his account of linguistic representation; it just means that the same principles are used in quite different domains.

\section{R9. Alternative approaches to linguistic representation}

Alongside structural priming, some commentators suggest further methods for studying linguistic representation. Ziegler et al. argue that priming is not special. If any method appears to relate to linguistic representation (e.g., multivoxel pattern analysis relating to thematic roles), then they assume that it can be used to constrain linguistic theory. This is reasonable in principle but, in practice, is likely to always face the problem that any effect could be due to the "parsing procedure P." For example, it might appear that the N400 in Event-related Potential (ERP) research could be diagnostic of semantic anomaly and, hence, semantic representation, whereas the P600 could be diagnostic of syntactic anomaly and, hence, syntactic representation. Whether an anomalous utterance in an ERP experiment produced a N400 or a P600 would then be informative about linguistic representation (e.g., Osterhout \& Mobley 1995 on anaphora). It turned out, however, that some semantically anomalous utterances produced a P600 (e.g., Kim \& Osterhout 2005). This finding means that these ERP components are not directly informative about linguistic representation. Rather than focus on one method, researchers might be able to combine findings from diverse methods and draw general conclusions about linguistic representation. But there is little sign of this happening, presumably because theoretical linguistics would ascribe any such findings to "P." Priming does not face this concern, in part because there is no need for an indirect intervening theory, but also because it has an established methodology that is largely consistently applied across studies (a property that it shares with acceptability judgments, of course).

We agree with Rowland \& Monaghan that multiple methods, including corpus research and novel word studies, are desirable in developmental research. Such methods, however, have important limitations for studying representation. It is unclear whether novel word studies, for example, are informative about underlying representations or about processing. We cannot tell whether children's reluctance to produce DO structures with novel verbs that they have previously encountered in PO structures is because they do not have an abstract (generalized) DO representation, or because they find it difficult to produce unusual word orders. In contrast, priming between DO sentences involving different verbs supports an abstract DO representation underlying these sentences (Rowland et al. 2012). As we note in R6, priming studies can address how representations change (e.g., whether a structure is lexically specific at time 1 but not at time 2 ).

With respect to nonexperimental methods, GonzalezMarquez et al., Lester et al., and Rowland \& Monaghan emphasize the potential offered by corpus and distributional analyses for both developmental and adult research (see also Rees \& Bott). These approaches offer valuable data about weak generative capacity and factors that may influence structural choices in language use (e.g., Bresnan et al. 2007) (see also sect. R10).
Recent advances in statistical analysis help researchers to exclude confounding variables. However, straightforward distributional analyses are less informative than the substantial corpus-based evidence of structural priming (see Gries \& Kootstra 2017) - an approach that also may address some of the concerns about statistical power highlighted in R2. Moreover, experimental and corpus-based evidence for priming complement each other. (Note that Kempson \& Gregoromichelaki and De Ruiter \& De Ruiter query whether syntactic priming occurs in conversation on the basis of the negative results of Healey et al. [2014]). But their conclusions contrast with many other demonstrations of priming in conversation (e.g., Gries 2005; Gries 2011; Myslín \& Levy 2016; Reitter \& Moore 2014; Szmrecsanyi 2006).

Hagoort, Martin et al., and Ziegler et al. propose that neuroimaging techniques can address many representational questions. As Hagoort notes, fMRI adaptation (based on variations in the BOLD response dependent on previous experience) is a form of priming and supports the segregation of semantic, syntactic, and lexical processing in ways that are shared across comprehension and production (Menenti et al. 2011; Segaert et al. 2012). But neuroimaging research not based on priming has so far been less informative about representation.

Cai \& Liu suggest that, although priming offers deeper insights than intuition-based data into the microscopic aspect of linguistic representation, it is less well suited to evaluating the macroscopic characteristics of language systems and, specifically, the interconnectivity of elements at each level. Complex network approaches may offer an interesting way to study system-level representational similarities (e.g., between native and non-native speakers) and changes in these systems (e.g., during L2 learning), in a way that can complement the insights from structural priming into the development of linguistic representations in particular populations (such as L2 learners), or in principle in individual speakers.

\section{R10. Details of our theory}

Our linguistic account assumes an architecture that distinguishes distinct semantic, syntactic and phonological representations. We agree with Hagoort that it is largely compatible with the Memory Unification and Control model (Hagoort 2014; Hagoort \& Indefrey 2014), a neurobiological model of language based on data from imaging studies that identifies discrete neuronal network contributions for unifying semantic, syntactic, and phonological information. However, various commentators dispute specific aspects of our account.

Ziegler et al. are right that there is less structural priming evidence relating to semantic structure than to syntactic structure. This fact reflects the greater interest historically in the latter than the former (e.g., Bock 1986). But it is not contentious that structural priming of semantic structure occurs and that it is informative for theories of semantic representation (Maldonado et al.; see also Bunger, Papafragou, and Trueswell 2013, for priming of event structure, and Rees \& Bott for extension to pragmatic representation). Furthermore, studies converge in supporting a lexically independent level of semantic representation that includes quantificational information. We anticipate that the growing interest in the use of structural priming to investigate semantic representation will give rise to more detailed theoretical proposals based on converging evidence from multiple studies, as has occurred for syntactic representation.

Ambridge, González-Márquez et al., Günther, Lester et al., and Ziegler et al. express some scepticism about a level of syntactic representation that is independent of semantic and lexical information. The finding that priming of syntactic structure is not enhanced when thematic mappings or semantic features are repeated (Bock \& Loebell 1990; Huang et al. 2016; Messenger et al. (2012b)) suggests that semantic information is not incorporated into syntactic structure. It specifically fits badly with the construction-based approach advocated by Günther, who suggests that 
priming effects might hold between entire constructions (which could be related but of different types), or just their syntactic (i.e., form) or their semantic (i.e., meaning) components. The straightforward interpretation of a construction grammar is that it involves form-meaning pairings and that these pairings can be primed. This is compatible with integrated syntax and semantics. Günther suggests that priming can occur between the form or the meaning side of a form-meaning pairing. But if so, this means there is autonomy of syntax. Such autonomy can explain priming between sentences with the same syntax but very different meanings (e.g., from locatives to passives; Bock \& Loebell 1990).

In our account, thematic relations and other semantic information are represented separately from syntactic structure and are mapped to syntactic representations (e.g., Chang et al. 2003; Köhne et al. 2014; Cai et al. 2012). Not surprisingly, some mappings are preferred over others (e.g., Bresnan et al. 2007; Ferreira 1994; Kempen \& Harbusch 2004). Such preferences, however, do not imply that semantic (or indeed, lexical) content is part of the syntactic representation. Moreover, acceptability judgments for sentences reflect their likelihood of occurrence (Bresnan 2007), so it is unsurprising that sentences involving preferred mappings are rated as more acceptable than sentences involving less preferred mappings (Ambridge). In fact, Ambridge's results suggest that acceptability judgments are affected by semantic factors, a point that reinforces our conclusion that acceptability judgments do not straightforwardly reflect syntactic representation.

With respect to the relationship between lexical content and syntactic structure, Ziegler et al. argue that function words can be a locus of priming, based on Ferreira's (2003) study. In fact, Ferreira argued for an autonomous, lexically independent syntactic component, consistent with our account: Participants did not produce structures that included complementizer that following primes that included complementizer that unless the primes also involved the same syntactic structure. In other words, repetition of the function word alone did not determine choice of structure (see also Bock 1989).

Similarly, our claim that syntactic representations are independent of closed class features is not undermined by Feldman \& Milin's finding of differences in priming of single word production (measured by response latencies) depending on the modality of the prime (drawing vs. word), and differences in the specific verb form that was produced. Any such effects do not appear to relate to structural priming (as far as is known; Pickering \& Branigan 1998).

Regarding open-class lexical content, priming evidence provides no support for redundant representations in which the same utterance (or part of an utterance) is represented both as a single lexicalized chunk and as the integration of several more schematic constructions (Günther). If this were the case, we might expect stronger priming from frequently encountered phrases (which should be more likely to be redundantly represented), as there would be two potential sources of priming (Rowland et al. 2012). Konopka and Bock (2009), however, found that idiomatic expressions and nonidiomatic expressions yielded equivalent priming.

Hartsuiker \& Bernolet suggest that the lexical boost to priming (which appears to be short lived) need not reflect a link between syntactic representations and the lexicon but may instead arise from an explicit memory of the prime that rapidly decays, with the verb acting as a retrieval cue. Malhotra et al. (2008) demonstrated (using a dynamical systems model) that our assumption of a link between syntactic representations and the lexicon is compatible with a short-lived lexical boost. The longevity of the lexical boost, therefore, does not discriminate between our account and alternative accounts. Furthermore, it does not matter whether explicit memory processes are involved in priming: Any such memories must still make reference to abstract structure (e.g., that the verb give appeared with an NP and an NP) to be able to explain priming between nonidentical sentences (Branigan \& McLean 2016). Note also that these memories cannot be based on specific wordforms (because the lexical boost is unaffected by repetition of specific features such as tense, aspect and number; Pickering \& Branigan 1998).

Other commentators discuss alternative ways of conceptualizing an abstract level of syntactic representation. Hudson supports our overall approach but suggests that priming evidence suggests that we should abandon the standard assumption that constituentstructure is basic, and with dependencies (e.g., subject-verb agreement) being defined with respect to constituent-structure. Instead, he treats dependency as basic and constituency as derivative at best. It seems most straightforward to us to define priming (e.g., of Prepositional Object vs. Double Object) in constituentstructure terms (e.g., priming VP $\rightarrow \mathrm{V}$ NP PP rather than $\mathrm{V} \rightarrow$ V NP NP), but it presumably would be possible to define it also in terms of dependencies, for instance from $\mathrm{V}$ to $\mathrm{N}$ (i.e., the head noun of the NP) and V to $\mathrm{P}$ (i.e., the head preposition of the $\mathrm{PP}$ ) rather than dependencies from $\mathrm{V}$ to $\mathrm{N}$ and $\mathrm{V}$ to $\mathrm{N}$. Of course, priming of subject-verb agreement itself (Haskell et al. 2010) fits particularly well with a dependency-based analysis. An analysis of priming in terms of a dependency-based grammar such as Word Grammar (Hudson 2010) would be very interesting.

Kempson \& Gregoromichelaki propose that our data do not need to be interpreted in terms of hierarchical representations such as those derived from context-free constraints (or similar), and interpret it in terms of sequential patterns of processing actions (see also O'Grady). If those patterns discriminate between linguistic structures that prime differently (e.g., the sequential pattern for POs differs from the pattern for DOs), then their approach may be able to capture priming data, and an analysis in terms of dynamic syntax also would be very interesting (Cann et al. 2005).

Adger and Koring \& Reuland (see also Gaston et al.) challenge our use of priming between unergatives and unaccusatives to propose that they have similar syntactic representations. In contrast, their favored accounts assume that unaccusatives have a very different structure from unergatives - a difference that would almost certainly preclude priming, given the sensitivity of priming to comparable syntactic differences (e.g., priming does not occur between constructions with different numbers of arguments). Koring \& Reuland simply assume an analysis involving NP-movement, but we query the assumptions that underlie analyses such as theirs. We do not dispute syntactic distinctions between types of subjects (though at least some relevant distinctions between unergatives versus unaccusatives may be semantic rather than syntactic; e.g., Zaenen 1988), but argue that these distinctions do not require different constituent-structure representations. Similarly, Adger assumes that a distinction between Lily froze solid and * Lily danced tired means that Lily froze and Lily danced must have different constituent structures. Instead, it shows that danced and froze have syntactic properties that allow them both to combine with just a subject but which have different effects on combination with a subject and another constituent. We cannot conclude that Lily froze and Lily danced have different constituent structures. Approaches such as those of Koring \& Reuland and Adger would lead to extremely complex constituent structures and are challenged by theories assuming flat structures (e.g., Culicover \& Jackendoff 2005; Pollard \& Sag 1994).

Our concern with many other experimental methods is that their results can be due to processing (i.e., processing theory "P" in Chomsky 1981). Therefore, we did not review all results (indeed, we did not have the space to do so). As an example, Bever and Sanz's (1997) finding of faster responses to a probe word associated with a subject noun that preceded an unaccusative compared to an unergative verb could be due to semantic differences between unaccusatives and unergatives (rather than reactivating an antecedent at an NP-trace) (see also Agnew et al. 2014). Both Koring \& Reuland and Adger point to many experimental studies that contrast unaccusative and unergative sentences, but none of them demonstrate that the distinctions are due to constituent structure differences. Many experimental methods such as studies using reactivation or visual world 
paradigms provide results relating to interpretation; there may be an underlying syntactic cause of the effects or there may not, but we typically cannot tell. In contrast, priming is informative about syntactic representation.

We also note that our description of "mainstream generative grammar" (Koring \& Reuland) is our best attempt to capture the key issues of a family of views and to ignore factional distinctions. It is not helpful to argue that we are using concepts that are obsolete within the authors' favored branch of linguistic theory. We are simply trying to describe a complex state of affairs and identify the core issues that we wish to dispute.

Kwon \& Lee argue that priming evidence provides only limited benefit compared with acceptability judgments. But criticising a new method on the grounds that it has not discovered as much as an old method would simply lead to stagnation. Their specific point about distinguishing linear and hierarchical relations is more insightful. We believe that priming data support a single level of structure that is specified for linear and hierarchical relations together, rather than one in which hierarchical structure is independently represented. For example, Bernolet et al. (2007) found priming between German and Dutch noun phrases that both have adjective-verb order (the $X$ that red is) but not between English and Dutch, presumably because English has verb-adjective order. In other words, the priming is not localized at an unordered hierarchical level (relative clause, unspecified for order) and, therefore, we propose that such an unordered level does not exist. In response to Hartsuiker \& Bernolet, the absence of priming for PO structures following shifted PO prime sentences is compatible with an account in which there is no priming of independent hierarchical relations, and thematic-function and thematic-order priming effects counteract each other (Cai et al. 2012).

Of course, we do not assume that syntax can be reduced to simple sequential structure (see also Gaston et al.). Otherwise, we would not assume constituent-structure representations such as those in Figure 1. Priming also cannot be reduced to linear order. For example, it occurs when the internal structure of prime and target constituents are different (e.g., complex vs. simple noun phrases in dative priming; see Fox Tree \& Meijer 1999; Pickering \& Branigan 1998), or when the critical alternation occurs at a different location in the prime and target - for example, datives in main versus subordinate clauses (Branigan et al. 2006) or noun phrases sentence-initially versus -finally (Melinger \& Cleland 2011).

Hartsuiker \& Bernolet and Francis question how we interpret the representation of coerced sentences. Hartsuiker \& Bernolet argue that the control (event) sentences could be given a coerced reading (i.e., both sentences could have a missing predicate represented). But the picture presented with the prime sentence forced it to have an event reading. Additionally, although coerced and control sentences did not differentially prime the production of full VP targets (relative to VP-primes such as began drinking the champagne), they did differentially prime the production of coerced targets, with coerced primes inducing more coerced targets than control primes. This finding further suggests that participants did not postulate a predicate for control primes. Francis proposes that the data of Raffray et al. (2014) might be due entirely to syntactic priming and so might indicate that the three sentence types (began the speech, began the champagne, began drinking the champagne) all have different syntactic structures. The problem with this explanation is that a purely syntactic account might argue that began the champagne had a covert $\mathrm{V}$ (corresponding to drinking) but began the speech could not have a covert $\mathrm{V}$-and if this is the case, began the champagne should behave more similarly than began the speech to began drinking the champagne, contrary to the findings of Raffray et al..

\section{R11. Further directions}

We argue in the target article that structural priming can be used to address outstanding linguistic controversies, and highlight some specific examples (sect. 3.1). Commentators identify interesting further cases that exemplify the potential of this method.

Francis discusses how priming might be used to clarify the syntactic representation of quantificational NPs and specifically whether they have the same representation as collective NPs. Assuming that an appropriate experiment can be designed (e.g., an alternative form for $a$ lot of papers), then any finding that $A$ bundle of sticks primes A lot of papers would suggest that quantificational NPs and collective NPs involve the same syntactic representation, in which the first noun acts as the head. We suspect that such priming would occur, because priming appears to be sensitive to categorical distinctions rather than featural distinctions (e.g., tense or number; Pickering \& Branigan 1998).

O'Grady highlights how priming could be used to test specific representational questions across typologically diverse languages. As we note in section 3.2, such studies can address fundamental questions about the diversity of linguistic representations and potential language universals. With respect to subject-verb sequences, it may well be possible to prime neither is versus are (for example) and demonstrate that such priming constitutes priming of subject-verb agreement, rather than simply priming of the verb form (see Haskell et al. 2010). Any such priming could be due to semantic rather than syntactic relations, as both types of dependency affect choice of verb form (cf. Bock et al. 1999). And even if the effects are syntactic, they could relate to a unit consisting of the whole sentence rather than one consisting of the subject and verb. For example, the locus of priming for neither are satisfactory could be $\mathrm{NP}_{+} \mathrm{vl}_{+\mathrm{pl}} \mathrm{AP}$ rather than $\mathrm{NP}_{+\mathrm{pl}} \mathrm{V}_{+\mathrm{pl}}$. We also suggest that priming different interpretations of Everyone didn't finish the project takes place at the semantic rather than syntactic level.

Rees \& Bott point to Bott and Chemla's (2016) finding that scalar implicatures can be primed (see also Maldonado et al.). We see this as a fascinating example of how priming can be extended to further linguistic domains, specifically diverse aspects of structure relating to interpretation. Priming of pragmatic representations is, of course, compatible with it being sensitive to all levels of linguistic representation.

Cai \& Liu and Kootstra \& Rossi suggest ways in which structural priming can be used to investigate bilingual representation. In particular, Kootstra \& Rossi note that priming can be used to study first language attrition. We agree: It could be used to determine whether an L1 had become completely inaccessible or not. Of course, it would be possible for a language to be temporarily inaccessible but to become accessible again later in life, and it might be possible to use priming to render an inaccessible language accessible again.

Finally, we support Hagoort's suggestion that fMRI adaptation offers promising directions for future research that may allow us to identify more precisely the type of priming that occurs, as different linguistic representations lead to adaptation in different brain areas. This approach may be particularly appropriate for studying structures that do not involve alternations. It is also likely to yield important findings about the neural basis of linguistic representation. It shows one way in which our proposals about the use of priming to understand linguistic representation may be extended and therefore underlines their value in the development of the cognitive sciences of language.

\section{ACKNOWLEDGMENTS}

This research was supported by the European Union's Seventh Framework Programme for research, technological development and demonstration under grant agreement no. 613465; the Economic and Social Research Council (grant number ESN0131151); and a Royal Society of Edinburgh/ National Science Foundation of China research grant. We thank Geoff Pullum and members of the Meaning and Grammar Research Group for their comments. All views expressed are our own. 


\section{References}

[The letters “ $a$ " and “ $r$ " before author's initials stand for target article and response references, respectively]

Adger, D. (2003) Core syntax: A minimalist approach, vol. 33. Oxford University Press. [JS]

Agnew, Z. K., van de Koot, H., McGettigan, C. \& Scott, S. K. (2014) Do sentences with unaccusative verbs involve syntactic movement? Evidence from neuroimaging. Language, Cognition and Neuroscience 29(9):1035-45. doi:10.1080/ 23273798.2014.887125. [LK, rHPB]

Alexiadou, A., Anagnostopoulou, E. \& Everaert, M. (2004) The unaccusativity puzzle. Oxford University Press. [DA]

Allan, K. (2006) Lexicon: Structure. In: Encyclopedia of language and linguistics, second edition, ed. K. Brown, pp. 148-51. Elsevier. [RH]

Allen, K., Ibara, S., Seymour, A., Cordova, N. \& Botvinick, M. (2010) Abstract structural representations of goal-directed behavior. Psychological Science 21 (10):1518-24. doi:10.1177/0956797610383434. [MCMD, rHPB]

Allen, M. L., Haywood, S., Rajendran, G. \& Branigan, H. (2011) Evidence for syntactic alignment in children with autism. Developmental Science 14(3):540 48. doi:10.1111/j.1467-7687.2010.01001.x. [aHPB]

Almeida, D. (2010) "Weak quantitative standards in linguistics research? The debate between Gibson/Fedorenko \& Sprouse/Almeida." Talking Brains blog, moderated by G. Hickok and D. Poeppel, June 14, 2010. Available at: http://www. talkingbrains.org/2010/06/weak-quantitative-standards-in.html. [PH]

Ambridge, B., Bidgood, A., Pine, J. M., Rowland, C. F. \& Freudenthal, D. (2016) Is passive syntax semantically constrained? Evidence from adult grammaticality judgment and comprehension studies. Cognitive Science 40(6):1435-59. doi: 10.1111/cogs.12277. [BA]

Ambridge, B. \& Rowland, C. F. (2013) Experimental methods in studying child language acquisition. Wiley Interdisciplinary Reviews: Cognitive Science 4 (2):149-68. doi:10.1002/wcs.1215. [aHPB]

Arai, M., van Gompel, R. P. G. \& Scheepers, C. (2007) Priming ditransitive structures in comprehension. Cognitive Psychology 54(3):218-50. doi:10.1016/j. cogpsych.2006.07.001. [arHPB, JZ]

Arunachalam, S. (2013) Experimental methods for linguists. Language and Linguistics Compass 7(4):221-32. [JZ]

Austin, P. \& Bresnan, J. (1996) Non-configurationality in Australian Aboriginal languages. Natural Language and Linguistic Theory 14(2):215-68. doi:10.1007/ BF00133684. [aHPB]

Baayen, R. H., Milin, P. \& Ramscar, M. (2016a) Frequency in lexical processing. Aphasiology 30(11):1174-220. [LBF]

Baayen, R. H., Shaoul, C., Willits, J. \& Ramscar, M. (2016b) Comprehension without segmentation: A proof of concept with naive discrimination learning. Language, Cognition, and Neuroscience. 31(1):106-28 [LBF]

Bard, E. G., Robertson, D. \& Sorace, A. (1996) Magnitude estimation of linguistic acceptability. Language 72(1):32-68. doi:10.2307/416793. [aHPB]

Bencini, G., Bock, K. \& Goldberg, A. (2002) How abstract is grammar? Evidence from structural priming in language production. Poster presented at the Fifteenth Annual Meeting of the CUNY Conference on Human Sentence Processing, New York, NY. [JZ]

Bencini, G. M. L. \& Valian, V. V. (2008) Abstract sentence representations in 3-yearolds: Evidence from language production and comprehension. Journal of Memory and Language 59(1):97-113. doi:10.1016/j.jml.2007.12.007. [arHPB]

Berg, T. (2009) Structure in language: A dynamic perspective. Routledge. [aHPB]

Bergen, B. \& Coulson, S. (2006) Frame-shifting humor in simulation-based language understanding. IEEE Intelligent Systems 21(2):59-62. [MG-M]

Berko, J. (1958) The child's learning of English morphology. WORD 14(2-3):150_ 77. [JZ]

Bernolet, S., Collina, S. \& Hartsuiker, R. J. (2016) The persistence of syntactic priming revisited. Journal of Memory and Language 91:99-116. [RJH]

Bernolet, S. \& Hartsuiker, R. J. (2010) Does verb bias modulate syntacticpriming? Cognition 114:455-61. [GJK]

Bernolet, S., Hartsuiker, R. J. \& Pickering, M. J. (2007) Shared syntactic representations in bilinguals: Evidence for the role of word-order repetition. Journal of Experimental Psychology: Learning, Memory, and Cognition 33(5):931-49. doi:10.1037/0278-7393.33.5.931. [arHPB]

Bernolet, S., Hartsuiker, R. J. \& Pickering, M. J. (2009) Persistence of emphasis in language production: A cross-linguistic approach. Cognition 112(2):300-17. doi:10.1016/j.cognition.2009.05.013. [aHPB, RJH]

Bernolet, S., Hartsuiker, R. J. \& Pickering, M. J. (2012) Effects of phonological feedback on the selection of syntax: Evidence from between-language syntactic priming. Bilingualism: Language and Cognition 15(3):503-16. doi:10.1017/ S1366728911000162. [aHPB]

Bernolet, S., Hartsuiker, R. J. \& Pickering, M. J. (2013) From language-specific to shared syntactic representations: The influence of second language proficiency on syntactic sharing in bilinguals. Cognition 127(3):287-306. doi:10.1016/j. cognition.2013.02.005. [rHPB]
Berwick, R. C. \& Weinberg, A. S. (1984) The grammatical basis of linguistic performance: Language use and acquisition. MIT Press. [arHPB]

Bever, T. G. (1970) The cognitive basis for linguistic structures. In: Cognition and Language Development, ed. R. Hayes, pp. 279-362. Wiley and Sons. https://doi org/10.1017/CBO9781107415324.004 [aHPB]

Bever, T. G. \& Sanz, M. (1997) Empty categories access their antecedents during comprehension: Unaccusatives in Spanish. Linguistic Inquiry 28:69-91. [LK, $\mathrm{rHPB}]$

Biederman, I. (1987) Recognition by components: A theory of human image understanding. Psychological Review 94(2):115-17. doi:10.1037/0033295X.94.2.115. [aHPB]

Biederman, I. \& Cooper, E. E. (1991) Priming contour-deleted images: Evidence for intermediate representations in visual object recognition. Cognitive Psychology 23(3):393-419. doi:10.1016/0010-0285(91)90014-F. [aHPB]

Bock, J. K. (1986) Syntactic persistence in language production. Cognitive Psychology 18(3):355-87. Available at: https://doi.org/10.1016/0010-0285(86)90004-6 [arHPB, DA, GJK, NK, MCMD]

Bock, K. (1989) Closed-class immanence in sentence production. Cognition 31 (2):163-86. doi:10.1016/0010-0277(89)90022-X. [arHPB, NK]

Bock, K., Dell, G. S., Chang, F. \& Onishi, K. H. (2007) Persistent structural priming from language comprehension to language production. Cognition 104(3):43758. doi:10.1016/j.cognition.2006.07.003. [aHPB, RR]

Bock, K. \& Griffin, Z. M. (2000) The persistence of structural priming: Transient activation or implicit learning? Journal of Experimental Psychology: General 129:177-92. doi:10.1037/0096-3445.129.2.177. [ASM, RR, LRS, $\mathrm{rHPB}]$

Bock, K. \& Loebell, H. (1990) Framing sentences. Cognition 35(1):1-39. doi:10.1016/0010-0277(90)90035-I. [arHPB, EJF, RK, KM]

Bock, K., Loebell, H. \& Morey, R. (1992) From conceptual roles to structural relations: Bridging the syntactic cleft. Psychological Review 99:150-71. doi:10.1037/0033-295X.99.1.150. [aHPB]

Bock, K. \& Miller, C. A. (1991) Broken agreement. Cognitive Psychology 23(1):4593. doi:10.1016/0010-0285(91)90003-7. [aHPB]

Bock, K., Nicol, J. L. \& Cutting, J. C. (1999) The ties that bind: Creating number agreement in speech. Journal of Memory and Language 40(3):330-46. doi:10.1006/jmla.1998.2616. [rHPB]

Borer, H. \& Wexler, K. (1987) The maturation of syntax. In Parameter Setting, ed. T Roeper \& E. Williams, pp. 123-72. D. Reidel. doi:10.1007/978-94-009-37277_6. [aHPB]

Borodkin, K., Kenett, Y. N., Faust, M. \& Mashal, N. (2016) When pumpkin is closer to onion than to squash: The structure of the second language lexicon. Cognition 156:60-70. [ZGC]

Bott, L. \& Chemla, E. (2016) Shared and distinct mechanisms in deriving linguistic enrichment. Journal of Memory and Language 91:117-40. doi:10.1016/j. jml.2016.04.004. [arHPB, MM, AR]

Boudreault, P. \& Mayberry, R. I. (2006) Grammatical processing in American Sign Language: Age of first-language acquisition effects in relation to syntactic structure. Language and Cognitive Processes 21(5):608-35. doi:10.1080/ 01690960500139363. [MLH]

Branigan, H. P. \& McLean, J. F. (2016) What children learn from adults' utterances: An ephemeral lexical boost and persistent syntactic priming in adult-child dialogue. Journal of Memory and Language 91:141-57. doi:10.1016/j. jml.2016.02.002. [arHPB]

Branigan, H. P., Pickering, M. J. \& Cleland, A. A. (1999) Syntactic priming in written production: Evidence for rapid decay. Psychonomic Bulletin \& Review 6 (4):635-40. Available at: http://doi.org/10.3758/BF03212972. [RR]

Branigan, H. P., Pickering, M. J. \& Cleland, A. A. (2000) Syntactic co-ordination in dialogue. Cognition 75(2):13-25. doi:10.1016/S0010-0277(99)00081-5. [arHPB]

Branigan, H. P., Pickering, M. J. \& McLean, J. F. (2005) Priming prepositionalphrase attachment during comprehension. Journal of Experimental Psychology: Learning, Memory, and Cognition 31(3):468-81. doi:10.1037/02787393.31.3.468 [arHPB, DA]

Branigan, H. P., Pickering, M. J., McLean, J. F. \& Cleland, A. A. (2007) Syntactic alignment and participant role in dialogue. Cognition 104:163-97. [LRS]

Branigan, H. P., Pickering, M. J., McLean, J. F. \& Stewart, A. J. (2006) The role of local and global syntactic structure in language production: Evidence from syntactic priming. Language and Cognitive Processes 21:974-1010. doi:10.1080/ 016909600824609. [arHPB]

Branigan, H. P., Pickering, M. J., Stewart, A. J. \& McLean, J. F. (2000) Syntactic priming in spoken production: Linguistic and temporal interference. Memory b Cognition 28(8):1297-302. Available at: http://doi.org/10.3758/BF03211830. [RR]

Breheny, R. (2008) A new look at the semantics and pragmatics of numerically quantified noun phrases. Journal of Semantics 25(2):93-139. [AR]

Brennan, J. \& Pylkkänen, L. (2016) MEG evidence for incremental sentence composition in the anterior temporal lobe. Cognitive Science 41(S6):1515-31. doi:10.1111/cogs.12445. [LK] 
Bresnan, J. (1975) Comparative deletion and constraints on transformations. Linguistic Analysis 1:25-74. [PG]

Bresnan, J. (2007) Is syntactic knowledge probabilistic? Experiments with the English dative alternation. In Roots: Linguistics in search of its evidential base, ed. S. Featherston \& W. Sternefeld, pp. 75-96. Mouton de Gruyter. [arHPB]

Bresnan, J., Cueni, A., Nikita, T. \& Baayen, R. H.(2007) Predicting the dative alternation. In: Cognitive foundations of interpretation, ed. G. Bouma, I Krämer \& J. Zwarts, pp. 69-94. Royal Netherlands Academy of Science. [rHPB]

Bresnan, J. \& Kaplan, R. (1982) Grammars as mental representations of language. In: The mental representation of grammatical relations, ed. J. Bresnan, pp. vii-lii. MIT Press. [aHPB]

Bunger, A., Papafragou, A. \& Trueswell, J. C. (2013) Event structure influences language production: Evidence from structural priming in motion event description. Journal of Memory and Language 69(3):299-323. doi:10.1016/j. jml.2013.04.002. [arHPB]

Burzio, L. (1981) Intransitive verbs and Italian auxiliaries. Unpublished doctoral dissertation. Department of Linguistics and Philosophy, MIT. [LK]

Bybee, J. L. (1994) A view of phonology from a cognitive and functional perspective. Cognitive Linguistics 5(4):285-305. [MG-M]

Bybee, J. L. (2006) From usage to grammar: The mind's response to repetition. Language 82:711-33. [NAL, MCMD]

Bybee, J. L. \& Moder, C. L. (1983) Morphological classes as natural categories. Language 59:251-89. [MG-M]

Cai, Z. G., Pickering, M. J. \& Branigan, H. P. (2012) Mapping concepts to syntax: Evidence from structural priming in Mandarin Chinese. Journal of Memory and Language 66(4):833-49. doi:10.1016/j.jml.2012.03.009. [arHPB, ZGC, JZ]

Cai, Z. G., Pickering, M. J. \& Sturt, P. (2013) Processing verb-phrase ellipsis in Mandarin Chinese: Evidence against the syntactic account. Language and Cognitive Processes 28:810-28. doi:10.1080/01690965.2012.665932. [aHPB $\mathrm{RJH}]$

Cai, Z. G., Pickering, M. J., Wang, R. \& Branigan, H. P. (2015) It is there whether you hear it or not: Syntactic representation of missing arguments. Cognition 136:255-67. doi:10.1016/j.cognition.2014.11.017. [aHPB]

Cai, Z. G., Pickering, M. J., Yan, H. \& Branigan, H. P. (2011) Lexical and syntactic representations in closely related languages: Evidence from Cantonese-Mandarin bilinguals. Journal of Memory and Language 65(4):431-45. doi:10.1016/j jml.2011.05.003. [aHPB]

Campbell, A. L. \& Tomasello, M. (2001) The acquisition of English dativeconstructions. Applied Psycholinguistics 22:253-67. Available at: https://doi.org/10. 1017/S0142716401002065. [CFR]

Cann, R., Kempson, R. \& Marten, L. (2005) The dynamics of language: An introduction. Elsevier. [RK, rHPB]

Carminati, M. N., van Gompel, R. P. G., Scheepers, C. \& Arai, M. (2008) Syntactic priming in comprehension: The role of argument order and animacy. Journal of Experimental Psychology: Learning, Memory, and Cognition 34(5):1098-1110. doi:10.1037/a0012795. [aHPB]

Čech, R. \& Mačutek, J. (2009) Word form and lemma syntactic dependency networks in Czech: A comparative study. Glottometrics 19:85-98. [ZGC]

Cesario, J. (2014) Priming, replication, and the hardest science. Perspectives on Psychological Science 9(1):40-8. [MCMD]

Chafe, W. (1976) Givenness, contrastiveness, definiteness, subjects and topics. In: Subject and topic, ed. C. N. Li, pp. 27-55. Academic Press. [aHPB]

Chafe, W. L. (1994) Discourse, consciousness, and time: The flow and displacement of conscious experience in speaking and writing. University of Chicago Press. [NAL]

Chamberlin, T. C. (1897) Studies for students: The method of multiple working hypotheses. The Journal of Geology 5(8):837-48. [JZ]

Champollion, L. (in press) Distributivity, collectivity and cumulativity. In: Wiley's companion to semantics, ed. L. Matthewson, C. Meier, H. Rullman \& T. E. Zimmermann. Wiley. [MM]

Chan, A. Y. (2004) Syntactic transfer: Evidence from the interlanguage of Hong Kong Chinese ESL learners. Modern Language Journal 88:56-74. [ZGC]

Chang, F., Bock, K. \& Goldberg, A. E. (2003) Can thematic roles leave traces of their places? Cognition 90(1):29-49. doi:10.1016/S0010-0277(03)00123-9. [arHPB, $\mathrm{RJH}, \mathrm{JZ}]$

Chang, F., Dell, G. S. \& Bock, K. (2006) Becoming syntactic. Psychological Review 113(2):234-72. Available at: https://doi.org/10.1037/0033-295X.113.2.234. [RJH, GJK, ASM, CFR, RR, LRS]

Chang, F., Janciauskas, M. \& Fitz, H. (2012) Language adaptation and learning: Getting explicit about implicit learning. Linguistics and Language Compass 6 (5):259-78. Available at: http://doi.org/10.1002/lnc3.337. [RR]

Chemla, E. \& Bott, L. (2014) Processing inferences at the semantics/pragmatics frontier: Disjunctions and free choice. Cognition 130(3):380-96. [MM]

Chemla, E. \& Bott, L. (2015) Using structural priming to study scopal representations and operations. Linguistic Inquiry 46(1):157-72. doi:10.1162/ling. [aHPB, $\mathrm{MM}, \mathrm{JZ}]$
Chen, B., Jia, Y., Wang, Z. \& Dunlap, S. (2013) Is word-order similarity necessary for cross-linguistic structural priming? Second Language Research 29:375-89.

Chierchia, G. (2013) Logic in grammar: Polarity, free choice, and intervention. Oxford University Press. [AR]

Chierchia, G., Fox, D. \& Spector, B. (2012) Scalar implicature as a grammatical phenomenon. In: An international handbook of natural language meaning, vol. 3, ed. P. Portner, C. Maienborn \& K. von Heusinger, pp. 2297-331. Mouton de Gruyter. [MM]

Chomsky, N. (1955/1975) The logical structure of linguistic theory. Plenum. [LK]

Chomsky, N. (1957) Syntactic structures. Walter de Gruyter. [aHPB]

Chomsky, N. (1965) Aspects of the Theory of Syntax, vol. 119. MIT Press. doi:10.1016/0732-118X(86)90008-5. [aHPB, NK]

Chomsky, N. (1977) On Wh-movement. In: Formal syntax, ed. A. Akmajian, P. Culicover \& T. Wasow, pp. 71-132. Academic Press. [NK]

Chomsky, N. (1980) Rules and representations. Columbia University Press. [aHPB]

Chomsky, N. (1981) Lectures on government and binding: The Pisa lectures. Walter de Gruyter/Foris/Mouton de Gruyter. [arHPB, DA, EJF]

Chomsky, N. (1986) Knowledge of language: Its nature, origin, and use. Greenwood Publishing Group/Praeger. [aHPB, LK]

Chomsky, N. (1995) The minimalist program. MIT Press. [aHPB, LK]

Chomsky, N. (2001) Derivation by phase. In: Ken Hale: A life in language, ed. M. Kenstowicz, pp. 1-52. MIT Press. [LK]

Cho-Reyes, S., Mack, J. E. \& Thompson, C. K. (2016) Grammatical encoding and learning in agrammatic aphasia: Evidence from structural priming. Journal of Memory and Language 91:202-18. [JZ]

Cho-Reyes, S. \& Thompson, C. (2012) Structural priming in agrammatic aphasia. Procedia-Social and Behavioral Sciences 61:259-61. doi:10.1016/j. sbspro.2012.10.176. [rHPB]

Clahsen, H. \& Felser, C. (2006) Grammatical processing in language learners. Applied Psycholinguistics 27(1):3-42. doi:10.1017/S0142716406060024. [aHPB, ZGC]

Clahsen, H. \& Muysken, P. (1986) The availability of universal grammar to adult and child learners - A study of the acquisition of German word order. Second Language Research 2(2):93-119. doi:10.1177/026765838600200201. [aHPB]

Cleland, A. A. \& Pickering, M. J. (2003) The use of lexical and syntactic information in language production: Evidence from the priming of noun-phrase structure. Journal of Memory and Language 49(2):214-30. doi:10.1016/S0749-596X(03) 00060-3. [aHPB, LBF, MLH]

Cleland, A. A. \& Pickering, M. J. (2006) Do writing and speaking employ the same syntactic representations? Journal of Memory and Language 54(2):185-98. doi:10.1016/j.jml.2005.10.003. [rHPB]

Cong, J. \& Liu, H. (2014) Approaching human language with complex networks. Physics of Life Reviews 11(4):598-618. [ZGC]

Cooper, R. (2012) Type theory and semantics in flux. In: Handbook of the philosophy of science, Vol. 14: Philosophy of linguistics, ed. R. Kempson, N. Asher \& T. Fernando, pp. 271-323. Elsevier. [RK, aHPB]

Conwell, E. \& Demuth, K. (2007) Early syntactic productivity: Evidence from dative shift. Cognition 103:163-79. doi:10.1016/j.cognition.2006.03.003. [CFR]

Corley, M. \& Scheepers, C. (2002) Syntactic priming in English sentence production: Categorical and latency evidence from an Internet-based study. Psychonomic Bulletin and Review 9(1):126-31. doi:10.3758/BF03196267. [aHPB]

Corominas-Murtra, B., Valverde, S. \& Solé, R. V. (2009) The ontogeny of scale-free syntax networks: Phase transitions in early language acquisition. Advances in Complex Systems 12:371-92. [ZGC]

Coulson, S. \& Van Petten, C. (2002) Conceptual integration and metaphor: An event-related potential study. Memory \& Cognition 30:958-68. [MG-M]

Covington, N. V. \& Duff, M. C. (2016) Expanding the language network: Direct contributions from the hippocampus. Trends in Cognitive Sciences 20(12):86970. $[\mathrm{RK}]$

Cowart, W. (1997) Experimental syntax: Applying objective methods to sentence judgments. Sage. [JS, JZ]

Crain, S. \& Thornton, R. (1998) Investigations in universal grammar: A guide to experiments on the acquisition of syntax and semantics. MIT Press. [LK]

Croft, W. (1995) Autonomy and functionalist linguistics. Language 71:490-532. [NAL]

Croft, W. (2001) Radical construction grammar: Syntactic theory in typological perspective. Oxford University Press. [aHPB, FG]

Croft, W. \& Poole, K. T. (2008) Inferring universals from grammatical variation: Multidimensional scaling for typological analysis. Theoretical Linguistics 34:137. [MG-M]

Culbertson, J. \& Gross, S. (2009) Are linguists better subjects? The British Journal for the Philosophy of Science 60(4):721-36. [JS]

Culicover, P. W. \& Jackendoff, R.(2005) Simpler syntax, vol. 10. Oxford University Press. doi:10.1093/acprof. [arHPB, EJF, PH, MM]

Dąbrowska, E. (2010) Naive v. Expert intuitions: An empirical study of acceptability judgments. Linguistic Review 27(1):1-23. doi:10.1515/tlir.2010.001. [JS, rHPB] 
Dąbrowska, E. (2014) Words that go together: Measuring individual differences in native speakers' knowledge of collocations. The Mental Lexicon 9(3):401-18. [MG-M]

De Bot, K. (2004) The multilingual lexicon: Modelling selection and control. International Journal of Multilingualism 1:17-32. [GJK]

De Bot, K., Broersma, M. \& Isurin, L. (2009) Sources of triggering in code switching. In: Multidisciplinary approaches to code switching, ed. L. Isurin, D. Winford \& K. de Bot, pp. 85-102. John Benjamins. [GJK]

De Groot, A. \& Hagoort, P., eds. (in press) Research methods in psycholinguistics and the neurobiology of language: A practical guide. Wiley Blackwell. [PH]

Dehaene, S., Naccache, L., Le Clec'H, G., Koechlin, E., Mueller, E., DehaeneLambertz, G., van de Moortele, P.-F. \& Le Bihan, D. (1998) Imaging unconscious semantic priming. Nature 395(6702):597-600. doi:10.1038/26967. [aHPB]

Dell, G., Chang, F. \& Griffin, Z. (1999) Connectionist models of language production: Lexical access and grammatical encoding. Cognitive Science 23(4):517-42. $[\mathrm{RH}]$

Dell, G. S. \& Chang, F. (2014) The P-chain: Relating sentence production and its disorders to comprehension and acquisition. Philosophical Transactions of the Royal Society B 369(1634):20120394. [GJK]

Dell, G. S. \& Ferreira, V. S. (2016) Structural priming. Special issue of the Journal of Memory and Language 91. [ASM]

Denison, D. (2010) Category change in English with and without structural change. In: Gradience, gradualness and grammaticalization, ed. E. C. Traugott \& G. Trousdale, pp. 105-28. John Benjamins. [EJF]

Diessel, H. (2015) Usage-based construction grammar. In: Handbook of cognitive linguistics, ed. E. Dąbrowska and D. Divjak, pp. 295-321. De Gruyter. [NAL]

Divjak, D. S. \& Arppe, A. (2013) Extracting prototypes from exemplars What can corpus data tell us about concept representation? Cognitive Linguistics 24 (2):221-74. [MG-M]

Du Bois, J. W. (2014) Towards a dialogic syntax. Cognitive Linguistics 25:359-410. [NAL]

Du Bois, J. W., Hobson, R. P. \& Hobson, J. A. (2014) Dialogic resonance and intersubjective engagement in autism. Cognitive Linguistics 25:411-41. [NAL]

Duran-Eppler, E. (2011) Emigranto. The syntax of German-English code-switching. Braumüller. $[\mathrm{RH}]$

Ellis, N. C. \& Ferreira-Junior, F. (2009) Constructions and their acquisition: Islands and the distinctiveness of their occupancy. Annual Review of Cognitive Linguistics 7:187-220. [NAL]

Elman, J., Bates, E., Johnson, M., Karmiloff-Smith, A., Parisi, D. \& Plunkett, K. (1996) Rethinking innateness: A connectionist perspective on development. MIT Press. [RH]

Erickson, L. C. \& Thiessen, E. D. (2015) Statistical learning in language: Theory, validity, and predictions of a statistical learning account of language acquisition. Developmental Review 37:66-108. [JZ]

Eshghi, A., Hough, J. \& Purver, M. (2013) Incremental grammar induction from child-directed dialogue utterances. In: Proceedings of the Fourth Annual Workshop on Cognitive Modeling and Computational Linguistics (CMCL), Sofia, Bulgaria, August 2013, pp. 94-103, ed. V. Demberg \& R. Levy. Association for Computational Linguistics. [RK]

Eshghi, A., Howes, C., Gregoromichelaki, E., Hough, J. \& Purver, M. (2015) Feedback in conversation as incremental semantic update. In: Proceedings of the 11th International Conference on Computational Semantics (IWCS 2015), pp. 261-71. Queen Mary University of London, UK, April 2015. [RK]

Eubank, L. (1996) Negation in early German-English interlanguage: More valueless features in the L2 initial state. Second Language Research 12(1):73-106. [ZGC]

Evans, N. \& Levinson, S. C. (2009) The myth of language universals: Language diversity and its importance for cognitive science. Behavioral and Brain Sciences 32(5):429-48. doi:10.1017/S0140525X0999094X. [aHPB]

Featherston, S. (2009) Relax, lean back, and be a linguist. Zeitschrift für Sprachwissenschaft 28(1):127-32. [JS]

Feiman, R. \& Snedeker, J. (2016) The logic in language: How all quantifiers are alike, but each quantifier is different. Cognitive Psychology 87:29-52. doi:10.1016/j. cogpsych.2016.04.002. [aHPB, MM, JZ]

Feist, M. I. (2008) Space between languages. Cognitive Science 32(7):1177-99. [MG-M]

Feist, M. I. (2010) Inside in and on: Typological and psycholinguistic perspectives. In: Language, Cognition, and Space, ed. V. Evans \& P. Chilton, pp. 95-114. Equinox. [MG-M]

Feist, M. I. (2013) Codability and cost in the naming of motion events. Rivista Italiana di Filosofia del Linguaggio 7(3):45-61. [MG-M]

Feist, M. I. \& Duffy, S. E. (2015) Moving beyond "Next Wednesday": The interplay of lexical semantics and constructional meaning in an ambiguous metaphoric statement. Cognitive Linguistics 26(4):633-56. [MG-M]

Feldman, L. B., Milin, P. \& Baayen, R. H. (2013a) Frequency and regularity effects do not interact in verb production. Presentation at European Society for Cognitive Psychology. Marseilles, France. August 30, 2013. [LBF]

Feldman, L. B., Milin, P. \& Baayen, R. H. (2013b) Frequency and regularity effects do not interact in verb production: A challenge to dual mechanism accounts of inflectional processing. Poster presented at Architectures and Mechanisms of Language Processing, Budapest, Hungary. August 22, 2013. [LBF]

Ferjan Ramirez, N., Lieberman, A. M. \& Mayberry, R. I. (2013) The initial stages of first-language acquisition begun in adolescence: When late looks early. Journal of Child Language 40(2):391-414. doi:10.1017/S0305000911000535. [MLH]

Ferreira, F. (1994) Choice of passive voice is affected by verb type and animacy. Journal of Memory and Language 33(6):715-36. doi:10.1006/jmla.1994.1034. [rHPB]

Ferreira, V. S. (2003) The persistence of optional complementizer production: Why saying "that" is not saying "that" at all. Journal of Memory and Language 48:379-98. doi:10.1016/S0749-596X(02)00523-5. [arHPB, GJK, JZ]

Ferreira, V. S. \& Bock, K. (2006) The functions of structural priming. Language and Cognitive Processes 21:1011-29. [GJK]

Ferreira, V. S., Bock, K., Wilson, M. P. \& Cohen, N. J. (2008) Memory for syntax despite amnesia. Psychological Science 19(9):940-46. doi:10.1111/j.14679280.2008.02180.x.Memory. [aHPB, RK]

Ferrer i Cancho, R., Solé, R. V. \& Köhler, R. (2004) Patterns in syntactic dependency networks. Physical Review E, 69:051915. [ZGC]

Fiengo, R. \& May, R. (1994) Indices and identity. MIT Press. [aHPB]

Fillmore, C. J. (1976) Frame semantics and the nature of language. Annals of the New York Academy of Sciences 280:20-32. [MG-M]

Fillmore, C. J., Kay, P. \& O'Connor, M. C. (1988) Regularity and idiomaticity in grammatical constructions: The case of let alone. Language 64(3):501-38. [MG-M]

Fine, A. B. \& Jaeger, T. F. (2016) The role of verb repetition in cumulative structural priming in comprehension. Journal of Experimental Psychology: Learning, Memory, and Cognition 42:1362-76. [RR]

Fine, A. B., Jaeger, T. F., Farmer, T. A. \& Qian, T. (2013) Rapid expectation adaptation during syntactic comprehension. PLOS ONE 8(10):e77661. doi:10.1371/journal.pone.0077661. [aHPB, RR]

Fisher, C. (2001) Partial sentence structure as an early constraint on language acquisition. In: Perception, cognition, and language: Essays in honor of Henry and Lila Gleitman, ed. B. Landau, J. Sabini, J. Jonides \& E. L. Newport, pp. 275-90. MIT Press. [aHPB]

Fisher, C. (2002) Structural limits on verb mapping: The role of abstract structure in 2.5-year-olds' interpretation of novel verbs. Developmental Science 5(1):55-64. [JZ]

Fleischer, Z., Pickering, M. J. \& McLean, J. F. (2012) Shared information structure Evidence from cross-linguistic priming. Bilingualism: Language and Cognition 15(3):568-79. doi:10.1017/S1366728911000551. [aHPB]

Flett, S. (2006) A comparison of syntactic representation and processing in first and second language production. Unpublished doctoral dissertation, University of Edinburgh. [aHPB, EJF, KM]

Flett, S., Branigan, H. P. \& Pickering, M. J. (2013) Are non-native structural preferences affected by native language preferences? Bilingualism: Language and Cognition 16(4):751-60. doi:10.1017/S1366728912000594. [aHPB]

Fodor, J. A., Bever, T. G. \& Garrett, M. F. (1974) The psychology of language: An introduction to psycholinguistics and generative grammar. McGraw-Hill. [aHPB]

Fodor, J. D. (1983) Phrase structure parsing and the island constraints. Linguistics and Philosophy 6(2):163-223. doi:10.1007/BF00635643. [aHPB]

Fodor, J. D. (1989) Empty categories in sentence processing. Language and Cognitive Processes 4:SI155-209. [aHPB]

Foltz, A., Thiele, K., Kahsnitz, D. \& Stenneken, P. (2015) Children's syntacticpriming magnitude: Lexical factors and participant characteristics. Journal of Child Language 42(4):932-45. doi:10.1017/S0305000914000488. [rHPB]

Forster, K. I. \& Davis, C. (1984) Repetition priming and frequency attenuation in lexical access. Journal of Experimental Psychology: Learning, Memory, and Cognition 10(4):680-98. doi:10.1037/0278-7393.10.4.680. [аНPB]

Fox Tree, J. E. \& Meijer, P. J. A. (1999) Building syntactic structure in speaking. Journal of Psycholinguistic Research 28(1):71-92. Available at: http://www.ncbi. nlm.nih.gov/pubmed/9949715. [arHPB]

Francis, E. J. \& Yuasa, E. (2008) A multi-modular approach to gradual change in grammaticalization. Journal of Linguistics 44:45-86. [EJF]

Franck, J., Soare, G., Frauenfelder, U. H. \& Rizzi, L. (2010) Object interference in subjectverb agreement: The role of intermediate traces of movement. Journal of Memory and Language 62(2):166-82. doi:10.1016/j.jml.2009.11.001. [aHPB, MCMD]

Frankland, S. M. \& Greene, J. D. (2015) An architecture for encoding sentence meaning in left mid-superior temporal cortex. Proceedings of the National Academy of Sciences of the United States of America 112(37):11732-37. doi:10.1073/pnas.1421236112. [JZ]

Fraundorf, S. H. \& Jaeger, T. F. (2016) Readers generalize adaptation to newly encountered dialectal structures to other unfamiliar structures. Journal of Memory and Language 91:28-58. [ASM]

Frazier, L. (1987) Sentence processing: A tutorial review. In: Attention and performance XII: The psychology of reading, ed. M. Coltheart, pp. 561-86. Erlbaum. [aHPB]

Frazier, L. \& Rayner, K.(1982) Making and correcting errors during sentence comprehension: Eye movements in the analysis of structurally ambiguous 
References/Branigan and Pickering: An experimental approach to linguistic representation

sentences. Cognitive Psychology 14(2):178-210. doi:10.1016/0010-0285(82) 90008-1. [aHPB]

Fricke, M. \& Kootstra, G. J. (2016) Primed code switching in spontaneous bilingual dialogue. Journal of Memory and Language 91:181-201. [GJK]

Friedmann, N., Taranto, G. Shapiro, L. P. \& Swinney, D. (2008) The leaf fell (the leaf): The online processing of unaccusatives. Linguistic Inquiry 39(3):355-77. [DA, LK]

Futrell, R., Mahowald, K. \& Gibson, E. (2015) Large-scale evidence of dependency length minimization in 37 languages. Proceedings of the National Academy of Sciences of the United States of America 112(33):10336-41. [RH]

Gámez, P. B., Shimpi, P. M., Waterfall, H. R. \& Huttenlocher, J. (2009) Priming a perspective in Spanish monolingual children: The use of syntactic alternatives. Journal of Child Language 36(2):269-90. doi:10.1017/S0305000908008945. [aHPB]

Garraffa, M., Coco, M. I. \& Branigan, H. P. (2015) Effects of immediate and cumulative syntactic experience in language impairment: Evidence from priming of subject relatives in children with SLI. Language Learning and Development 11:18-40. doi:10.1080/15475441.2013.876277. [arHPB]

Garrett, M. F. (1975) The analysis of sentence production. In: The psychology of learning and motivation, vol. 9, ed. G. H. Bower, pp. 133-77. Academic Press. [aHPB, RJH]

Gazdar, G. (1981) Unbounded dependencies and coordinate structure. Linguistic Inquiry 12(2):155-84. [aHPB]

Gazdar, G., Klein, E., Pullum, G. K. \& Sag, I. A. (1985) Generalized phrase structure grammar. Harvard University Press. [aHPB]

Gelman, A. \& Carlin, J. (2014) Beyond power calculations assessing type S (sign) and type M (magnitude) errors. Perspectives on Psychological Science 9(6):641-51. [JS]

Gibson, E. \& Fedorenko, E. (2013) The need for quantitative methods in syntax and semantics research. Language and Cognitive Processes 28(1):88-124. doi:10.1080/01690965.2010.515080. [aHPB]

Gibson, E. \& Hickok, G. (1993) Sentence processing with empty categories. Language and Cognitive Processes 8(2):147-61. doi:10.1080/01690969308406952. [aHPB]

Gibson, T. \& Fedorenko, E. (2010) Weak quantitative standards in linguistics research. Trends in Cognitive Sciences 14(6):233-34. [PH]

Gildea, D. \& Temperley, D. (2010) Do grammars minimize dependency length? Cognitive Science 34:286-310. [RH]

Gillespie, M. \& Pearlmutter, N. J. (2013) Against structural constraints in subjectverb agreement production. Journal of Experimental Psychology: Learning, Memory, and Cognition 39(2):515-28. doi:10.1037/a0029005. [aHPB]

Gisborne, N. (2010) The event structure of perception verbs. Oxford University Press. [RH]

Givón, T., ed. (1983) Topic continuity in discourse: A quantitative cross-language study. Benjamins. [NAL]

Goldberg, A. E. (1995) Constructions: A construction grammar approach to argument structure. University of Chicago Press. [aHPB, FG, MLH]

Goldberg, A. E. (2003) Constructions: A new theoretical approach to language. Trends in Cognitive Sciences 7(5):219-24. Available at: http://dx.doi.org/10. 1016/S1364-6613(03)00080-9 [FG, MG-M]

Goldberg, A. E. (2006) Constructions at work: The nature of generalization in language. Oxford University Press. [aHPB, EJF, FG, NAL, CFR]

Goldinger, S. D., Luce, P. A. \& Pisoni, D. B. (1989) Priming lexical neighbors of spoken words: Effects of competition and inhibition. Journal of Memory and Language 28(5):501-18. doi:10.1016/0749-596X(89)90009-0. [аHPB]

Goldin-Meadow, S., So, W. C., Ozvïrek, A. \& Mylander, C. (2008) The natural order of events: How speakers of different languages represent events nonverbally. Proceedings of the National Academy of Sciences of the United States of America 105(27):9163-68. doi:10.1073/pnas.0710060105. [aHPB]

Green, D. W. \& Abutalebi, J. (2013) Language control in bilinguals: The adaptive control hypothesis. Journal of Cognitive Psychology 25:515-30. [GJK]

Gregoromichelaki, E. (2013) Grammar as action in language and music. In: Language, music and interaction, ed. M. Orwin, C. Howes \& R. Kempson, pp. 93-134. College Publications. [RK

Gregoromichelaki, E. (2017) Quotation in dialogue. In: The semantics and pragmatics of quotation, ed. P. Saka \& M. Johnson. Springer. [RK]

Gregoromichelaki, E. \& Kempson, R. (2013) The role of intentions in dialogue processing. In: Perspectives on linguistic pragmatics, ed. A. Capone, F. Lo Piparo \& M. Carapezza, pp. 185-216. Springer. [RK]

Gregoromichelaki, E. \& Kempson, R. (2015) Joint utterances and the (split-)turn taking puzzle. In: Interdisciplinary studies in pragmatics, culture and society, ed. J. L. Mey \& A. Capone, pp. 703-43. Springer. [RK]

Gregoromichelaki, E. \& Kempson, R. (forthcoming) Procedural syntax. In: Relevance: Pragmatics and interpretation, ed. K. Scott, B. Clark \& R. Carston. Cambridge University Press. [RK]

Gregoromichelaki, E., Kempson, R, Howes, C. \& Eshghi, A. (2013) On making syntax dynamic: The challenge of compound utterances and the architecture of the grammar. In: Alignment in communication: Towards a new theory of communication, ed. I. Wachsmuth, J. de Ruiter, P. Jaecks \& S. Kopp, pp. 57-86. John Benjamins. [RK]

Gregoromichelaki, E., Kempson, R., Purver, M., Mills, G. \& Cann, R. (2011)

Incrementality and intention-recognition in utterance processing. Dialogue and Discourse 2(1). [RK]

Grice, H. P. (1975) Logic and conversation. In: Speech acts, ed. P. Cole \& J. L. Morgan, pp. 41-58. Academic Press. [AR]

Gries, S. Th. (2005) Syntactic priming: A corpus-based approach. Journal of Psycholinguistic Research 34(4):365-99. doi:10.1007/s10936-005-6139-3. [NAL, $\mathrm{rHPB}]$

Gries, S. Th. (2011) Studying syntactic priming in corpora: implications of different levels of granularity. In: Converging evidence: Methodological and theoretical issues for linguistic research, ed. D. Schönefeld, pp. 143-65. John Benjamins. [rHPB]

Gries, S. Th. \& Kootstra, G. J.(2017) Structural priming within and across languages: A corpus-based perspective. Bilingualism: Language and Cognition 20(2):23550. doi: 10.1017/S1366728916001085. [rHPB]

Griffin, Z. M. \& Weinstein-Tull, J. (2003) Conceptual structure modulates structural priming in the production of complex sentences. Journal of Memory and Language 49(4):537-55. doi:10.1016/j.jml.2003.08.002. [aHPB, EJF]

Grill-Spector, K., Henson, R. \& Martin, A. (2006) Repetition and the brain: Neural models of stimulus-specific effects. Trends in Cognitive Sciences 10(1):14-23. [PH]

Gropen, J., Pinker, S., Hollander, M., Goldberg, R. \& Wilson, R. (1989) The learnability and acquisition of the dative alternation in English. Language 65 (2):203-57. [JZ]

Grosjean, F. (2010) Bilingual: Life and reality. Harvard University Press. [GJK]

Günther, F. (2016) Constructions in cognitive contexts: Why individuals matter in linguistic relativity research. de Gruyter Mouton. [FG]

Hagoort, P. (2005) On Broca, brain, and binding: A new framework. Trends in Cognitive Sciences 9:416-23. [PH]

Hagoort, P. (2014) Nodes and networks in the neural architecture for language: Broca's region and beyond. Current Opinion in Neurobiology 28:136-41. doi:10.1016/j.conb.2014.07.013. [AEM, PH, rHPB]

Hagoort, P. \& Indefrey, P. (2014) The neurobiology of language beyond single words. Annual Review of Neuroscience 37(1):347-62. doi:10.1146/annurevneuro-071013-013847. [PH, rHPB]

Hale, K. (1983) Warlpiri and the grammar of non-configurational languages. Natural Language and Linguistic Theory 1(1):5-47. doi:10.1007/BF00210374. [aHPB]

Hale, K. \& Keyser, J. (1993) On argument structure and the lexical expression of syntactic relations. In: The view from building 20, ed. K. Hale \& J. Keyser, pp. 53-109. MIT Press. [aHPB]

Hale, K. \& Keyser, J. (2002) Prolegomenon to a theory of argument structure. MIT Press. [aHPB]

Hall, M. L., Ferreira, V. S. \& Mayberry, R. I. (2012) Phonological similarity judgments in ASL: Evidence for maturational constraints on phonetic perception in sign. Sign Language and Linguistics 15(1):104-27. [MLH]

Hall, M. L., Ferreira, V. S. \& Mayberry, R. I. (2014) Investigating constituent order change with elicited pantomime: A functional account of SVO emergence. Cognitive Science 38(5):943-72. doi:10.1111/cogs.12105. [aHPB]

Hall, M. L., Ferreira, V. S. \& Mayberry, R. I. (2015) Syntactic priming in American Sign Language. PLoS ONE 10(3):e0119611. doi:10.1371/journal.pone.0119611. [MLH, rHPB]

Halliday, M. A. K. (1967) Notes on transitivity and theme in English: Part 2. Journal of Linguistics 3(2):199-244. doi:10.1017/S0022226700016613. [aHPB]

Hankamer, J. (1979) Deletion in coordinate structures. Garland Press. [aHPB]

Hare, M. L. \& Goldberg, A. E. (1999) Structural priming: Purely syntactic? In Proceedings of the Twenty-first Annual Meeting of the Cognitive Science Society, ed. M. Hahn \& S. C. Stones, pp. 208-11. Erlbaum. [NK, NAL, JZ]

Hartsuiker, R. J., Beerts, S., Loncke, M., Desmet, T. \& Bernolet, S. (2016) Crosslinguistic structural priming in multilinguals: Further evidence for shared syntax. Journal of Memory and Language 90:14-30. [ZGC]

Hartsuiker, R. J., Bernolet, S., Schoonbaert, S., Speybroeck, S. \& Vanderelst, D. (2008) Syntactic priming persists while the lexical boost decays: Evidence from written and spoken dialogue. Journal of Memory and Language 58(2):214-38. doi:10.1016/j.jml.2007.07.003. [aHPB, RJH, RR]

Hartsuiker, R. J. \& Kolk, H. H. (1998) Syntactic facilitation in agrammatic sentence production. Brain and Language 62(2):221-54. doi:10.1006/brln.1997.1905. [aHPB]

Hartsuiker, R. J. \& Pickering, M. J. (2008) Language integration in bilingual sentence production. Acta Psychologica 128:479-89. [GJK]

Hartsuiker, R. J., Pickering, M. J. \& Veltkamp, E. (2004) Is syntax separate or shared between languages? Cross-linguistic syntactic priming in Spanish-English bilinguals. Psychological Science 15(6):409-14. doi:10.1111/j.09567976.2004.00693.x. [aHPB, ZGC]

Hartsuiker, R. J. \& Westenberg, C. (2000) Word order priming in written and spoken sentence production. Cognition 75(2):B27-39. doi:10.1016/S0010-0277(99) 00080-3. [aHPB, NK] 
Haskell, T. R., Thornton, R. \& MacDonald, M. C. (2010) Experience and grammatical agreement: Statistical learning shapes number agreement production. Cognition 114(2):151-64. Available at: http://doi.org/10.1016/j.cognition.2009. 08.017. [rHPB]

Haspelmath, M. (2008) Frequency vs. iconicity in explaining grammatical asymmetries. Cognitive Linguistics 19:1-33. [MG-M]

Häussler, J., Juzek, T. \& Wasow, T. (2016) To be grammatical or not to be grammatical - is that the question? Poster presented at the Annual Meeting of the Linguistic Society of America, Washington, DC, January 7-10. [JS]

Haxby, J. V., Gobbini, M. I., Furey, M. L., Ishai, A., Schouten, J. L. \& Pietrini, P. (2001) Distributed and overlapping representations of faces and objects in ventral temporal cortex. Science 293:2425-29. [JZ]

Healey, P. G. T, Purver, M. \& Howes, C. (2014) Divergence in dialogue. PLoS ONE 9(2):e98598. doi:10.1371/journal.pone.0098598. [JPdR, RK, rHPB]

Heim, I. \& Kratzer, A. (1998) Semantics in generative grammar. Blackwell. [MG-M]

Hofmeister, P., Jaeger, T. F., Arnon, I., Sag, I. A. \& Snider, N. (2013) The source ambiguity problem: Distinguishing the effects of grammar and processing on acceptability judgments. Language and Cognitive Processes 28(1-2):48-87. doi:10.1080/01690965.2011.572401. [aHPB]

Hofmeister, P. \& Sag, I. A. (2010) Cognitive constraints and island effects. Language 86(2):366. [JS]

Hopkins, Z., Yuill, N. \& Keller, B. (2016) Children with autism align syntax in natural conversation. Applied Psycholinguistics 37(2):347-70. doi:10.1017/ S0142716414000599. [aHPB]

Horgan, D. (1976) The development of the full passive. Journal of Child Language 5:65-80. doi:10.1017/S030500090000194X. [aHPB]

Horn, L. (1972) On the semantic properties of logical operators in English. Unpublished doctoral dissertation. Department of linguistics, University of California, Los Angeles. [AR]

Hough, J. (2015) Modelling incremental self-repair processing in dialogue. Doctoral thesis. Queen Mary University of London. [RK]

Hough, J. \& Purver, M. (2017) Probabilistic record type lattices for incremental reference processing. In: Modern perspectives in type-theoretical semantics, ed. S. Chatzikyriakidis \& Z. Luo, pp. 189-222. Springer. [RK]

Huang, C.-T. J., Li, Y.-H. A. \& Li, Y. (2009) The syntax of Chinese. Cambridge University Press. [aHPB]

Huang, J., Pickering, M. J., Yang, J., Wang, S. \& Branigan, H. P. (2016) The independence of syntactic processing in Mandarin: Evidence from structural priming. Journal of Memory and Language 91:81-98. doi:10.1016/j. jml.2016.02.005. [arHPB]

Hudson, R. (2007) Language networks: The new word grammar. Oxford University Press. $[\mathrm{RH}]$

Hudson, R. (2010) An introduction to word grammar. Cambridge University Press. [RH, rHPB]

Huette, S., Winter, B., Matlock, T., Ardell, D. \& Spivey, M. J. (2014) Eye movements during listening reveal spontaneous grammatical processing. Frontiers in Psychology 5:410. doi: 10.3389/fpsyg.2014.00410. [MG-M]

Huttenlocher, J., Vasilyeva, M. \& Shimpi, P. (2004) Syntactic priming in young children. Journal of Memory and Language 50(2):182-95. doi:10.1016/j. jml.2003.09.003. [aHPB]

Ivanova, I., Branigan, H. P., McLean, J. F., Costa, A. \& Pickering, M. J. (2017) Do you what I say? People reconstruct the syntax of anomalous utterances. Language, Cognition and Neuroscience 32(2):175-89. [LRS]

Ivanova, I., Pickering, M. J., Branigan, H. P., Costa, A. \& McLean, J. F. (2012a) The comprehension of anomalous sentences: Evidence from structural priming. Cognition 122(2):193-209. doi:10.1016/j.cognition.2011.10.013. [aHPB]

Ivanova, I., Pickering, M. J., McLean, J. F., Costa, A. \& Branigan, H. P. (2012b) How do people produce ungrammatical utterances? Journal of Memory and Language 67:355-70. doi:10.1016/j.jml.2012.06.003. [arHPB]

Ivanova, I., Wardlow, L., Gollan, T. H. \& Ferreira, V. (2013) The (un)automaticity of syntactic processing in language production. Twenty-sixth Annual CUNY Conference on Human Sentence Processing, Columbia, SC. [LRS]

Jackendoff, R. (2002) Foundations of language: Brain, meaning, grammar, evolution. Oxford University Press. [aHPB, MG-M, PH]

Jackendoff, R. (2007) Linguistics in cognitive science: The state of the art. Linguistic Review 24(4):347-401. doi:10.1515/TLR.2007.014. [aHPB]

Jackendoff, R. (2013) Constructions in the parallel architecture. In: The Oxford Handbook of Construction Grammar, ed. T. Hoffmann \& G. Trousdale, pp. 7092. Oxford University Press. [NAL]

Jackendoff, R. (in press) Mental representations for language. In: Human language: From genes and brains to behavior, ed. P. Hagoort. MIT Press. [PH]

Jaeger, T. F. (2010) Redundancy and reduction: Speakers manage syntactic information density. Cognitive Psychology 61:23-62. [NAL]

Jaeger, T. F. \& Snider, N. (2007) Implicit learning and syntactic persistence: Surprisal and cumulativity. University of Rochester working papers in the language sciences 3:26-44. [GJK]
Jaeger, T. F. \& Snider, N. E. (2013) Alignment as a consequence of expectation adaptation: Syntactic priming is affected by the prime's prediction error given both prior and recent experience. Cognition 127(1):57-83. [GJK, NAL, RR]

Jiang, J. \& Liu, H. (2015) The effects of sentence length on dependency distance, dependency direction and the implications - based on a parallel EnglishChinese dependency treebank. Language Sciences 50:93-104. [RH]

Kaiser, E. (2012) Taking action: A cross-modal investigation of discourse-level representations. Frontiers in Psychology 3:156. Available at: https://doi.org/10. 3389/fpsyg.2012.00156. [MCMD]

Kamide, Y. (2012) Learning individual talkers' structural preferences. Cognition 124 (1): 66-71. Available at: http://doi.org/10.1016/j.cognition.2012.03.001. [RR]

Kantola, L. \& van Gompel, R. P. G. (2011) Between- and within-language priming is the same: Evidence for shared bilingual syntactic representations. Memory and Cognition 39(2):276-90. doi:10.3758/s13421-010-0016-5. [aHPB]

Kaplan, R. \& Bresnan, J. (1982) Lexical functional grammar: A formal system for grammatical representation. In The mental representation of grammatical relations, ed. J. Bresnan, pp. 173-281. MIT Press. [aHPB]

Kaschak, M. P. \& Glenberg, A. M. (2004) This construction needs learned. Journal of Experimental Psychology: General 133(3):450-67. doi:10.1037/00963445.133.3.450. [arHPB]

Kaschak, M. P., Kutta, T. J. \& Coyle, J. M. (2014) Long and short term cumulative structural priming effects. Language and Cognitive Processes 29(6):1-23. Available at: http://doi.org/10.1080/01690965.2011.641387. [RR]

Kaschak, M. P., Kutta, T. J. \& Jones, J. L. (2011a) Structural priming as implicit learning: Cumulative priming effects and individual differences. Psychonomic Bulletin \& Review 18(6):1133-39. Available at: http://doi.org/10.14440/jbm. 2015.54.A. [MCMD, RR]

Kaschak, M. P., Kutta, T. J. \& Schatschneider, C. (2011b) Long-term cumulative structural priming persists for (at least) one week. Memory \& Cognition 39 (3):381-88. Available at: http://doi.org/10.3758/s13421-010-0042-3. [LRS, RR]

Katz, J. J. (1981) Language and other abstract objects. Blackwell. [aHPB]

Ke, J. \& Yao, Y. (2008) Analysing language development from a network approach. Journal of Quantitative Linguistics 15(1):70-99. [ZGC]

Keenan, E. L. (1984) Semantic correlates of the ergative/absolutive distinction. Linguistics 22:197-224. [NAL]

Kempen, G. \& Harbusch, K. (2004) A corpus study into word order variation in German subordinate clauses: Animacy affects linearization independently of grammatical function assignment. In: Multidisciplinary approaches to language production, ed. T. Pechmann \& C. Habel, pp. 173-81. Mouton de Gruyter. [rHPB]

Kempen, G. \& Hoenkamp, E. (1987) An incremental procedural grammar for sentence formulation. Cognitive Science 11:201-58. [RJH]

Kempson, R., Cann, R., Gregoromichelaki, E. \& Chatzikyriakidis, S. (2016) Language as mechanisms for interaction. Theoretical linguistics 42(3-4):203-76. doi:10.1515/tl-2016-0011. [RK]

Kempson, R., Cann, R., Gregoromichelaki, E. \& Chatzikyriakidis, S. (2017) Actionbased grammar. Theoretical Linguistics 43(1-2):141-67. Published Online: 2017-06-09. DOI: https://doi.org/10.1515/tl-2017-0012 [RK]

Kempson, R., Meyer-Viol, W. \& Gabbay, D. (1999) VP ellipsis: Toward a dynamic, structural account. In: Fragments: Studies in ellipsis and gapping, ed. S. Lappin \& E. Benmamoun, pp. 227-89. Oxford University Press. [aHPB]

Kempson, R., Meyer-Viol, W. \& Gabbay, D. (2001) Dynamic syntax: The flow of language understanding. Blackwell. [RK]

Kidd, E. (2012) Individual differences in syntactic priming in language acquisition. Applied Psycholinguistics 33(02):393-418. Available at: https://doi.org/10.1017/ S0142716411000415. [MCMD]

Kim, A. \& Osterhout, L. (2005) The independence of combinatory semantic processing: evidence from event-related potentials. Journal of Memory and Language 52(2):205-25. doi:10.1016/j.jml.2004.10.002. [arHPB]

King, J. R. \& Dehaene, S. (2014) Characterizing the dynamics of mental representations: the temporal generalization method. Trends in Cognitive Sciences 18 (4):203-10. [AEM]

Kluender, R. \& Kutas, M. (1993) Subjacency as a processing phenomenon. Language and Cognitive Processes 8(4):573-633. [JS]

Knoeferle, P. (2014) Conjunction meaning can modulate parallelism facilitation: Eye-tracking evidence from German clausal coordination. Journal of Memory and Language 75:140-58. doi:10.1016/j.jml.2014.05.002. [rHPB]

Köhne, J., Pickering, M. J. \& Branigan, H. P. (2014) The relationship between sentence meaning and word order: Evidence from structural priming in German. Quarterly Journal of Experimental Psychology 67(2):304-18. doi:10.1080/17470218.2013.807855. [arHPB]

Konopka, A. E. \& Bock, K. (2009) Lexical or syntactic control of sentence formulation? Structural generalizations from idiom production. Cognitive Psychology 58(1):68-101. doi:10.1016/j.cogpsych.2008.05.002. [arHPB, FG, NK]

Koranda, M. J., Bulgarelli, F., Weiss, D. J. \& MacDonald, M. C. (2016) Parallels between action priming and syntactic priming. Poster presented at the International Workshop on Language Production. La Jolla, CA. [MCMD] 
Koornneef, A., Avrutin, S., Wijnen, F. \& Reuland, E. (2011) Tracking the preference for bound-variable dependencies in ambiguous ellipses and only-structures. In: Experiments at the interfaces, ed. J. Runner, pp. 67-101. Syntax and Semantics 37. Emerald Group Publishing Limited. [LK]

Koornneef, A. \& Reuland, E. (2016) On the shallow processing (dis)advantage: Grammar and economy. Frontiers in Psychology 7:82. doi:10.3389/ fpsyg.2016.00082. [LK]

Kootstra, G. J., van Hell, J. G. \& Dijkstra, T. (2009) Two speakers, one dialogue: An interactive alignment perspective on code-switching in bilingual speakers. In: Multidisciplinary approaches to code switching, ed. L. Isurin, D. Winford \& K de Bot, pp. 129-60. John Benjamins. [GJK]

Kootstra, G. J., van Hell, J. G. \& Dijkstra, T. (2010) Syntactic alignment and shared word order in code-switched sentence production: Evidence from bilingual monologue and dialogue. Journal of Memory and Language 63:210-31. [GJK]

Kootstra, G. J., van Hell, J. G. \& Dijkstra, T. (2012) Priming of code-switching in sentences: The role of lexical repetition, cognates, and proficiency. Bilingualism: Language and Cognition 15:797-819. [GJK]

Kootstra, G. J., van Hell, J. G. \& Dijkstra, T. (in revision). Interactive alignment drives lexical triggering of code-switching in bilingual dialogue. [GJK]

Koring, L., Mak, P. \& Reuland, E. (2012) The time course of argument reactivation revealed: Using the visual world paradigm. Cognition 123(3):361-79. [LK]

Kriegeskorte, N., Mur, M. \& Bandettini, P. (2008) Representational similarity analysis - connecting the branches of systems neuroscience. Frontiers in Systems Neuroscience 2:4-10. [PH]

Kroll, J. F., Bobb, S. C. \& Wodniecka, Z. (2006) Language selectivity is the exception, not the rule: Arguments against a fixed locus of language selection in bilingual speech. Bilingualism: Language and Cognition 9:119-35. [GJK]

Kübler, S., McDonald, R. \& Nivre, J. (2009) Dependency parsing. Synthesis Lectures on Human Language Technologies 2:1-127. [RH]

Kutas, M. \& Hillyard, S. A. (1980) Reading senseless sentences: Brain potentials reflect semantic incongruity. Science 207(4427):203-205. doi:10.1126/ science.7350657. [aHPB]

Lakoff, G. (1987) Women, fire, and dangerous things: What categories reveal about the mind. University of Chicago Press. [MG-M]

Lakoff, G. (1990) The invariance hypothesis: Is abstract reason based on imageschemas? Cognitive Linguistics 1(1):39-74. [MG-M]

Lambrecht, K. (1994) Information structure and sentence form: Topic, focus, and the mental representations of discourse referents. Cambridge University Press. [aHPB]

Langacker, R. (1969) On pronominalization and the chain of command. In: Modern studies in English, ed. D. A. Reibel, S. A. Schane \& E. Cliffs, pp. 160-86. Prentice-Hall. [NK]

Langacker, R. W. (1987) Foundations of cognitive grammar: Vol. 1. Theoretical prerequisites. Stanford University Press. [FG]

Langacker, R. W. (2000) A dynamic usage-based model. In: Usage-based models of language, ed. M. Barlow \& S. Kemmer, pp. 1-63. CSLI Publications. [FG]

Langacker, R. W. (2008) Cognitive grammar: A basic introduction. Oxford University Press. $[\mathrm{FG}]$

Langendoen, D. T. \& Postal, P. M. (1984) The vastness of natural languages. Basil Blackwell. [aHPB]

Larson, R. K. 1988. On the double object construction. Linguistic Inquiry 19 (3):335-91. Available at: http://www.jstor.org/stable/25164901. [rHPB]

Lau, E. F. \& Ferreira, F. (2005) Lingering effects of disfluent material on comprehension of garden path sentences. Language and Cognitive Processes 20 (5):633-66. [LRS]

Ledoux, K., Traxler, M. J. \& Swaab, T. Y. (2007) Syntactic priming in comprehension: Evidence from event-related potentials. Psychological Science 18(2):13543. doi:10.1111/j.1467-9280.2007.01863.x. [aHPB]

Lee, J. \& Thompson, C. K. (2011) Real-time production of unergative and unaccusative sentences in normal and agrammatic speakers: An eye tracking study. Aphasiology 25(6-7):813-25. [DA]

Leonard, L., Miller, C. A., Grela, B., Holland, A. L., Gerber, E. \& Petucci, M. (2000) Production operations contribute to the grammatical morpheme limitations of children with specific language impairment. Journal of Memory and Language 43(2):362-78. doi:10.1006/jmla.1999.2689. [aHPB]

Lester, N. A., Feldman, L. B. \& Moscosodel Prado Martín, F. (2017) You can take a noun out of syntax...: Syntactic similarity effects in lexical priming. In: Proceedings of the Thirty-ninth Annual Meeting of the Cognitive Science Society, ed. G. Gunzelmann, A. Howes, T. Tenbrink \& E. Davelaar, pp. 2537-42. Cognitive Science Society. [NAL]

Lester, N. A. \& Moscoso del Prado Martín, F. (2016) Syntactic flexibility in the noun: Evidence from picture naming. In: Proceedings of the Thirty-eighth Annual Conference of the Cognitive Science Society, ed. A. Papafragou, D. Grodner, D. Mirman \& J. C. Trueswell, pp. 2585-90. Cognitive Science Society. [NAL]

Levelt, W. J. M. (1972) Some psychological aspects of linguistic data. Linguistische Berichte 17:18-30. [aHPB]

Levelt, W. J. M. (1989) Speaking: Fromintention to articulation. MIT Press. [aHPB, LK]
Levin, B. \& Rappaport, M. (1995) Unaccusativity: At the syntax-lexical semantics interface. MIT Press. [PG]

Levinson, S. C. \& Meira, S. (2003) "Natural concepts" in the spatial topological domain - Adpositional meanings in crosslinguistic perspective: An exercise in semantic typology. Language 79:485-516. [MG-M]

Lewis, S. \& Phillips, C. (2015) Aligning grammatical theories and language processing models. Journal of Psycholinguistic Research 44:27-46. doi:10.1007/ s10936-014-9329-z. [aHPB]

Link, G. (1987) Generalized quantifiers and plurals. In: Generalized quantifiers: Linguistic and logical approaches, ed. P. Gardenfors, pp. 151-80. Reidel. [MM]

Liu, H. (2009) Statistical properties of Chinese semantic networks. Chinese Science Bulletin 54:2781-85. [ZGC]

Liu, H. \& Li, W. W. (2010) Language clusters based on linguistic complex networks. Chinese Science Bulletin 55(30):3458-65. [ZGC]

Liu, N. \& Bergen, B. (2016) When do language comprehenders mentally simulate locations? Cognitive Linguistics 27(2):181-203. [MG-M]

Loebell, H. \& Bock, K. (2003) Structural priming across languages. Linguistics 41 (5):791-824. doi:10.1515/ling.2003.026. [aHPB]

Long, D. L. \& Prat, C. S. (2008) Individual differences in syntactic ambiguity resolution: Readers vary in their use of plausibility information. Memory and Cognition 36(2):375-91. Available at: http://doi.org/10.3758/MC.36.2.375. [RR]

Luka, B. J. \& Barsalou, L. W. (2005) Structural facilitation: Mere exposure effects for grammatical acceptability as evidence for syntactic priming in comprehension. Journal of Memory and Language 52(3):444-67. doi:10.1016/j.jml.2005.01.013. [arHPB, RR]

Lupyan, G. (2012) Linguistically modulated perception and cognition: The label feedback hypothesis. Frontiers in Cognition 3(54):1-13. doi:10.3389/ fpsyg.2012.00054. [MG-M]

Lupyan, G. \& Casasanto, D. (2015) Meaningless words promote meaningful categorization. Language and Cognition 7(2):167-93. [MG-M]

MacDonald, M. C. (2013) How language production shapes language form and comprehension. Frontiers in Psychology 4:226. Available at: https://doi.org/10. 3389/fpsyg.2013.00226. [MCMD]

MacDonald, M. C., Pearlmutter, N. J. \& Seidenberg, M. S. (1994) The lexical nature of syntactic ambiguity resolution. Psychological Review 101(4):676-703. doi:10.1037/0033-295X.101.4.676. [aHPB]

Macmillan, N. A. \& Creelman, C. D. (2004) Detection theory: A user's guide. Psychology Press. [AEM]

Mahowald, K., Graff, P., Hartman, J. \& Gibson, E. (2016a) SNAP judgments: A small $\mathrm{N}$ acceptability paradigm (SNAP) for linguistic acceptability judgments. Language 92(3):619-35. [JS]

Mahowald, K., James, A., Futrell, R. \& Gibson, E. (2016b) A meta-analysis of syntactic priming in language production. Journal of Memory and Language 91:5-27. doi:10.1016/j.jml.2016.03.009. [JPdR, KM, ASM, RR, rHPB]

Maldonado, M., Chemla, E. \& Spector, B. (2017) Priming plural ambiguities. Journal of Memory and Language 95:89-101. [MM].

Malhotra, G., Pickering, M., Branigan, H. \& Bednar, J. A. (2008) On the persistence of structural priming: Mechanisms of decay and influence of word-forms. In: Proceedings of the Thirtieth Annual Conference of the Cognitive Science Society, Austin, TX, pp. 657-62, ed. B. C. Love, K. McRae \& V. M. Sloutsky. Cognitive Science Society. [rHPB]

Maratsos, M., Fox, D. E. C., Becker, J. A. \& Chalkley, M. A. (1985) Semantic restrictions on children's passives. Cognition 19(2):167-91. doi:10.1016/00100277(85)90017-4. [aHPB]

Marslen-Wilson, W., Tyler, L. K., Waksler, R. \& Older, L. (1994) Morphology and meaning in the English mental lexicon. Psychological Review 101:3-33. doi:10.1037/0033-295X.101.1.3. [arHPB]

Martin, A. E. (2016) Language processing as cue integration: Grounding the psychology of language in perception and neurophysiology. Frontiers in Psychology 7:1-17. [AEM]

Martin, A. E., \& Doumas, L. A. A. (2017) A mechanism for the cortical computation of hierarchical linguistic structure. PLoS Biology 15(3):e2000663. [AEM]

Matthews, P. (2007) Syntactic relations: A critical survey. Cambridge University Press. [aHPB]

May, R. (1985) Logical form: Its structure and derivation. MIT Press. [aHPB]

Mayberry, R. I., Chen, J. K., Witcher, P. \& Klein, D. (2011) Age of acquisition effects on the functional organization of language in the adult brain. Brain and Language 119(1):16-29. doi:10.1016/J.Bandl.2011.05.007. [MLH]

Mayberry, R. I. \& Lock, E. (2003) Age constraints on first versus second language acquisition: Evidence for linguistic plasticity and epigenesis. Brain and Language 87(3):369-84. doi:10.1016/S0093-934x(03)00137-8. [MLH]

Mayberry, R. I., Lock, E. \& Kazmi, H. (2002) Linguistic ability and early language exposure. Nature 417(6884):38. doi:10.1038/417038a. [MLH]

McDaniel, D. \& Cairns, H. S. (1998) Eliciting judgments of grammaticality and reference. In: Methods for assessing children's syntax, ed. D. McDaniel, C. McKee \& H. S. Cairns, pp. 233-54. MIT Press. [aHPB] 
McDonough, K. \& Mackey, A. (2006) Responses to recasts: Repetitions, primed production, and linguistic development. Language Learning 56(4):693-720. doi:10.1111/j.1467-9922.2006.00393.x. [ZGC, rHPB]

McElree, B. (2006) Accessing recent events. Psychology of Learning and Motivation 46:155-200. [AEM]

McElree, B. \& Bever, T. G. (1989) The psychological reality of linguistically defined gaps. Journal of Psycholinguistic Research 18(1):21-35. doi:10.1007/ BF01069044. [aHPB]

McGregor, W. (2009) Typology of ergativity. Language and Linguistics Compass 3:480-508. doi:10.1111/j.1749-818X.2008.00118.x. [WOG]

McMahon, L. E. (1963) Grammatical analysis as part of understanding a sentence. Unpublished doctoral dissertation, Harvard University. [aHPB]

McNamara, T. P. (2005) Semantic priming: Perspectives from memory and word recognition. Taylor \& Francis. [aHPB]

Mehler, A., Lücking, A., Banisch, S., Blanchard, P. \& Job, B. (2015) Towards a theoretical framework for analyzing complex linguistic networks. Springer. [ZGC]

Mel'čuk, I. (2009) Dependency in natural language. In: Dependency in linguistic description, ed. A. Polguère \& I. Mel’̌̌uk, pp. 1-110. John Benjamins. [RH]

Melinger, A. \& Cleland, A. A. (2011) The influence of sentential position on noun phrase structure priming. Quarterly Journal of Experimental Psychology 64 (11):2211-35. doi:10.1080/17470218.2011.586709. [arHPB]

Melinger, A. \& Dobel, C. (2005) Lexicallydriven syntactic priming. Cognition 98(1): B11-20. [LRS]

Menenti, L., Gierhan, S. M. E., Segaert, K. \& Hagoort, P. (2011) Shared language: Overlap and segregation of the neuronal infrastructure for speaking and listening revealed by functional MRI. Psychological Science 22(9):1173-82. doi:10.1177/0956797611418347. [arHPB, PH]

Merchant, J. (2001) The syntax of silence: Sluicing, islands and the theory of ellipsis. Oxford University Press. [aHPB]

Messenger, K., Branigan, H. P. \& McLean, J. F. (2011) Evidence for (shared) abstract structure underlying children's short and full passives. Cognition 121 (2):268-74. doi:10.1016/j.cognition.2011.07.003. [arHPB]

Messenger, K., Branigan, H. P. \& McLean, J. F. (2012a) Is children's acquisition of the passive a staged process? Evidence from six- and nine-year-olds' production of passives. Journal of Child Language 39(5):991-1016. [aHPB, BA]

Messenger, K., Branigan, H. P., McLean, J. F. \& Sorace, A. (2012b) Is young children's passive syntax semantically constrained? Evidence from syntactic priming. Journal of Memory and Language 66(4):568-87. doi:10.1016/j. jml.2012.03.008. [arHPB, BA, NK]

Meyer, D. E. \& Schvaneveldt, R. W. (1971) Facilitation in recognizing pairs of words: Evidence of a dependence between retrieval operations. Journal of Experimental Psychology 90(2):227-34. doi:10.1037/h0031564. [aHPB]

Milin, P., Feldman, L. B., Ramscar, M., Hendrix, P. \& Baayen, R. H. (2017) Discrimination in lexical decision. PLoS ONE 12(2):e0171935. Available at: https://doi.org/10.1371/journal.pone.0171935. [LBF]

Miller, C. A. \& Deevy, P. (2006) Structural priming in children with and without specific language impairment. Clinical Linguistics and Phonetics 20(5):387-99. doi:10.1080/02699200500074339. [aHPB]

Miller, G. A. (1962) Some psychological studies of grammar. American Psychologist 17(11):748-62. [aHPB]

Miller, G. A. \& McKean, K. O. (1964) A chronometric study of some relations between sentences. Quarterly Journal of Experimental Psychology 16(4):297308. doi:10.1080/17470216408416385. [aHPB]

Mol, L., Krahmer, E., Maes, A. \& Swerts, M. (2012) Adaptation in gesture: Converging hands or converging minds? Journal of Memory and Language 66 (1):249-64. doi:10.1016/j.jml.2011.07.004. [aHPB]

Monaghan, P. \& Rowland, C. F. (2017) Combining language corpora with experimental and computational approaches for language acquisition research. Language Learning 67(S1):14-39. Available at: https://doi.org/10.1111/lang.12221. [CFR]

Myers, J. (2009) The design and analysis of small-scale syntactic judgment experiments. Lingua 119:425-44. doi:10.1016/j.lingua.2008.09.003. [JZ]

Myslín, M. \& Levy, R. (2016) Comprehension priming as rational expectation for repetition: Evidence from syntactic processing. Cognition 147:29-56. doi:10.1016/j.cognition.2015.10.021. [RK, ASM, rHPB]

Naigles, L. (1990) Children use syntax to learn verb meanings. Journal of Child Language 17(2):357-74. [JZ]

Nicol, J. \& Swinney, D. (1989) The role of structure in coreference Assignment during sentence comprehension. Journal of Psycholinguistic Research 18(1):519. doi:10.1007/BF01069043. [aHPB]

Nieuwland, M. S., Martin, A. E. \& Carreiras, M. (2013) Event-related brain potential evidence for animacy processing asymmetries during sentence comprehension. Brain and Language 126(2):151-58. doi:10.1016/j.bandl.2013.04.005. [aHPB, $\mathrm{AEM}$

Ninio, A. (2006) Language and the learning curve: A new theory of syntactic development. Oxford University Press. [RH]
Noppeney, U. \& Price, C. J. (2004) An fMRI study of syntactic adaptation. Journal of Cognitive Neuroscience 16(4):702-13. doi:10.1162/089892904323057399. [aHPB]

Novick, J. M., Kim, A. \& Trueswell, J. C. (2003) Studying the grammatical aspects of word recognition: Lexical priming, parsing, and syntactic-ambiguity resolution Journal of Psycholinguistic Research 32:57-75. [NAL]

Núñez, R. \& Sweetser, E. (2006) With the future behind them: Convergent evidence from Aymara language and gesture in the crosslinguistic comparison of spatial construals of time. Cognitive Science 30(3):401-50. [MG-M]

O'Grady, W. (2015) Anaphora and the case for emergentism. In: The handbook of language emergence, ed. B. MacWhinney \& W. O'Grady, pp. 100-22. WileyBlackwell. doi:10.1002/9781118346136.ch4. [WOG]

Osterhout, L. \& Holcomb, P. J. (1992) Event-related brain potentials elicited by syntactic anomaly. Journal of Memory and Language 31(6):785-806. doi:10.1016/0749-596X(92)90039-Z. [aHPB]

Osterhout, L. \& Mobley, L. A. (1995) Event-related brain potentials elicited by failure to agree. Journal of Memory and Language 34(6):739-73. doi:10.1006/ jmla.1995.1033. [rHPB]

Pappert, S. \& Pechmann, T. (2013) Bidirectional structural priming across alternations: Evidence from the generation of dative and benefactive alternation structures in German. Language and Cognitive Processes 28(9):1303-22. doi:10.1080/01690965.2012.672752. [aHPB]

Pappert, S. \& Pechmann, T. (2014) Priming word order by thematic roles: No evidence for an additional involvement of phrase structure. Quarterly Journal of Experimental Psychology 67(11):2260-78. doi:10.1080/17470218.2014.918632. $[\mathrm{aHPB}, \mathrm{RJH}]$

Payne, T. E. (2006) Exploring language structure: A student's guide. Cambridge University Press. [aHPB]

Percival, K. (1990) Reflections on the history of dependency notions in linguistics. Historiographia Linguistica 17:29-47. [RH]

Perlmutter, D. M. (1978) Impersonal passives and the unaccusative hypothesis. In Proceedings of the Fourth Annual Meeting of the Berkeley Linguistics Society, ed. J. J. Jaeger, A. C. Woodbury, F. Ackerman, C. Chiarello, O. D. Gensler, J Kingston, E. E. Sweetser, H. Thompson \& K. W. Whistler, pp. 157-89. University of California, Berkeley Linguistics Society. [LK]

Perry, L. \& Lupyan, G. (2014) The role of language in multi-dimensional categorization: Evidence from transcranial direct current stimulation and exposure to verbal labels. Brain and Language 135:66-72. [MG-M]

Pesetsky, D. (1995) Zero syntax. MIT Press. [PG]

Peter, M., Chang, F., Pine, J. M., Blything, R. \& Rowland, C. F. (2015) When and how do children develop knowledge of verb argument structure? Evidence from verb bias effects in a structural priming task. Journal of Memory and Language 81:1-15. doi:10.1016/j.jml.2014.12.002. [aHPB, CFR]

Phillips, C. (2003) Linear order and constituency. Linguistic Inquiry 34:37-90. [PG]

Phillips, C. (2006) The real-time status of island phenomena. Language 82(4):795823. [JS]

Phillips, C. (2009) Should we impeach armchair linguists? In: Japanese/Korean Linguistics, vol 17, ed. S. Iwasaki, H. Hoji, P. M. Clancy \& S.-O. Sohn, pp. 49-64. CSLI Publications, University of Chicago Press. [aHPB, JS]

Pickering, M. \& Barry, G. (1991) Sentence processing without empty categories. Language and Cognitive Processes 6:229-59. [aHPB, RH]

Pickering, M. \& Barry, G. (1993) Dependency categorial grammar and coordination. Linguistics 31:855-902. [rHPB]

Pickering, M. J. \& Branigan, H. P. (1998) The representation of verbs: Evidence from syntactic priming in language production. Journal of Memory and Language 39(4):633-51. [arHPB, LBF, NK]

Pickering, M. J. \& Branigan, H. P. (1999) Syntactic priming in language production. Trends in Cognitive Sciences 3:136-41. [NAL]

Pickering, M. J., Branigan, H. P. \& McLean, J. F. (2002) Constituent structure is formulated in one stage. Journal of Memory and Language 46(3):586-605. doi:10.1006/jmla.2001.2824. [aHPB, RJH, NK]

Pickering, M. J. \& Ferreira, V. S. (2008) Structural priming: A critical review. Psychological Bulletin 134(3):427-59. Available at: http://dx.doi.org/10.1037/00332909.134.3.427 [a,rHPB, FG]

Pickering, M. J. \& Garrod, S. (2004) Toward a mechanistic psychology of dialogue. Behavioral and Brain Sciences 27:169-90. [AR]

Pickering, M. J. \& Garrod, S. (2014) Self-, other-, and joint monitoring using forward models. Frontiers in Human Neuroscience 8:132. [AR]

Pickering, M. J. \& Garrod, S. (2017) Priming and language change. In: The changing English language: Psycholinguistic perspectives, ed. M. Hundt, S. Mollin \& S. E. Pfenninger, pp. 173-90. Cambridge University Press. [rHPB]

Pickering, M. J., McLean, J. F. \& Branigan, H. P. (2013) Persistent structural priming and frequency effects during comprehension. Journal of Experimental Psychology: Learning, Memory, and Cognition 39(3):890-97. doi:10.1037/ a0029181. [rHPB]

Pinker, S. (1984) Language learnability and language development. Harvard University Press. Available at: https://doi.org/10.1007/s13398-014-0173-7.2. [CFR] 
Pinker, S. (1989) Learnability and cognition: The acquisition of argument structure. MIT Press. [aHPB]

Pinker, S., Lebeaux, D. S. \& Frost, L. A. (1987) Productivity and constraints in the acquisition of the passive. Cognition 26(3):195-267. [BA]

Polinsky, M. (2009) What agreement can do for you: First and last conjunct agreement in Tsez. Paper presented at the Annual Meeting of the Linguistic Society of America, San Francisco, CA. [WOG]

Pollard, C. \& Sag, I. A. (1994) Head-driven phrase structure grammar, vol. 8. University of Chicago Press. [arHPB, RJH]

Pothos, E. M. (2007) Theories of artificial grammar learning. Psychological Bulletin 133(2):227-44. [JZ]

Potter, M. C. \& Lombardi, L. (1998) Syntactic priming in immediate recall of sentences. Journal of Memory and Language 38(3):265-82. [aHPB]

Prasada, S. \& Pinker, S. (1993) Generalization of regular and irregular morphological patterns. Language and Cognitive Processes 8:1-56. [JZ]

Price, C. J. (2010) The anatomy of language: A review of $100 \mathrm{fMRI}$ studies published in 2009. Annals of the New York Academy of Sciences 1191:62-88. doi:10.1111/ j.1749-6632.2010.05444.x. [aHPB]

Purver, M., Eshghi, A. \& Hough, J. (2011) Incremental semantic construction in a dialogue system. In: Proceedings of the Ninth International Conference on Computational Semantics, ed. J. Bos \& S. Pulman, pp. 365-9. The Digital Library of The Association of Computational Linguistics. Available at: http://dl. acm.org/citation.. $\mathrm{cfm}$ ?id=2002669\&picked=prox. [RK]

Purver, M., Gregoromichelaki, E., Meyer-Viol, W. \& Cann, R. (2010) Splitting the "I"s and crossing the "you"s: Context, speech acts and grammar. In: Proceedings of the Fourteenth Workshop on the Aspects of Semantics and Pragmatics of Dialogue. ed. P. Lupkowski \& M. Purver, pp. 43-50. Polish Society for Cognitive Science (Poznań). [RK]

Raffray, C. N. \& Pickering, M. J. (2010) How do people construct logical form during language comprehension? Psychological Science 21(8):1090-97. doi:10.1177/ 0956797610375446. [aHPB, MM, JZ]

Raffray, C. N., Pickering, M. J., Cai, Z. G. \& Branigan, H. P. (2014) The production of coerced expressions: Evidence from priming. Journal of Memory and Language 74:91-106. doi:10.1016/j.jml.2013.09.004. [a,rHPB, EJF, RJH]

Ramscar, M., Dye, M. \& McCauley, S. M. (2013) Error and expectation in language learning: The curious absence of mouses in adult speech. Language 89 (4):760-93. [LBF]

Reber, A. S. (1967) Implicit learning of artificial grammars. Journal of Verbal Learning and Verbal Behavior 6(6):855-63. [JZ]

Reed, A. V. (1973) Speed-accuracy trade-off in recognition memory. Science 181 (4099):574-76. [AEM]

Rees, A. \& Bott, L. (2015) The role of the alternative in the derivation of scalar implicatures. Work presented at XPRAG 2015, Chicago, IL. [MM, AR]

Rees, A. \& Bott, L. (2016) Priming implicit communication. Work presented at Architectures and Mechanisms of Language Processing 2016, September 1-3, 2016, Bilbao, Spain. [AR]

Reinhart, T. (1983) Anaphora and semantic interpretation. University of Chicago Press. [NK]

Reisberg, D. (2007) Cognition. Exploring the science of the mind, third media edition. Norton. [RH]

Reitter, D. \& Moore, J. D. (2014) Alignment and task success in spoken dialogue. Journal of Memory and Language 76:29-46. doi:10.1016/j.jml.2014.05.008. [rHPB]

Reuland, E. (2011a) Anaphora and language design. MIT Press. [LK]

Reuland, E. (2011b) Syntax and interpretation systems: How is their labor divided? In: The Oxford Handbook of Linguistic Minimalism, ed. C. Boeckx, pp. 377-96. Oxford University Press. [LK]

Riches, N. G. (2012) Sentence repetition in children with specific language impairment: An investigation of underlying mechanisms. International Journal of Speech Language and Hearing Research 47(5):499-510. [aHPB]

Rosenbaum, D. A., Cohen, R. G., Meulenbroek, R. G. \& Vaughan, J. (2006) Plans for grasping objects. In: Motor control and learning over the lifespan, ed. M. Latash $\&$ F. Lestienne, pp. 9-25. Springer. [MCMD]

Rossi, E. (2013) Modulating the sensitivity to syntacticfactors in production: Evidence from syntactic priming in agrammatism. Applied Psycholinguistics 36:639-69. [GJK]

Rossi, E., Prystauka, Y. \& Diaz, M. (in revision) Investigating Ll attrition and language change: neuroimaging perspectives. In: Handbook of language attrition: Psycho- and neurolinguistic perspectives, ed. B. Köpke \& M. Keijzer. Oxford University Press. [GJK]

Rostoft, M. S., Sigmundsson, H., Whiting, H. T. A. \& Ingvaldsen, R. P. (2002) Dynamics of hand preference in 4 year-old children. Behavioural Brain Research 132:59-68. [MCMD]

Rowland, C. F., Chang, F., Ambridge, B., Pine, J. M. \& Lieven, E. V. M. (2012) The development of abstract syntax: Evidence from structural priming and the lexical boost. Cognition 125(1):49-63. doi:10.1016/j.cognition.2012.06.008 [arHPB, ZGC, CFR]

Rowland, C. F. \& Noble, C. L. (2011) The role of syntactic structure in children's sentence comprehension: Evidence from the dative. Language Learning and Development 7(1):55-75. Available at: https://doi.org/10.1080/ 15475441003769411. [CFR]

Ryskin, R. A., Qi, Z., Duff, M. C. \& Brown-Schmidt, S. (2017) Verb biases are shaped through lifelong learning. Journal of Experimental Psychology: Learning, Memory, and Cognition 43(5):781-94. [RR]

Sadock, J. M. (1991) Autolexical syntax: A theory of parallel grammatical representations. University of Chicago Press. [EJF]

Saffran, E. M. \& Martin, N. (1997) Effects of structural priming on sentence production in aphasics. Language and Cognitive Processes 12(5-6):877-82. doi:10.1080/016909697386772. [aHPB]

Saffran, J. R., Hauser, M. D., Seibel, R., Kapfhamer, J., Tsao, F. \& Cushman, F. (2008) Grammatical pattern learning by human infants and cotton-top tamarin monkeys. Cognition 107:479-500. [JZ]

Sag, I. A. \& Fodor, J. D. (1994) Extraction without traces. In: Proceedings of the Thirteenth Annual Meeting of the West Coast Conference on Formal Linguistics, ed. R. Aranovich, W. Byrne, S. Preuss \& M. Senturia, pp. 365-84. SLA, CSLI Publications. [aHPB]

Saj, A., Fuhrman, O., Vuilleumier, P. \& Boroditsky, L. (2014) Patients with left spatial neglect also neglect the "left side" of time. Psychological Science 25 (1):207-14. [MG-M]

Salamoura, A. \& Williams, J. N. (2006) Lexical activation of cross-language syntactic priming. Bilingualism 9(3):299-307. doi:10.1017/S1366728906002641. [aHPB]

Salamoura, A. \& Williams, J. N. (2007) Processing verb argument structure across languages: Evidence for shared representations in the bilingual lexicon. Applied Psycholinguistics 28(4):627-60. doi:10.1017/S0142716407070348. [aHPB, JZ]

Santesteban, M., Pickering, M. J., Laka, I. \& Branigan, H. P. (2015) Effects of casemarking and head position on language production? Evidence from an ergative OV language. Language, Cognition and Neuroscience 30(9):1175-86. doi:10.1080/23273798.2015.1065335. [aHPB, WOG]

Sato, Y. (2011) Local ambiguity, search strategies and parsing in dynamic syntax. In: The dynamics of lexical interfaces, ed. E. Gregoromichelaki \& C. Howes, pp. 205-34. CSLI Publications. [RK]

Savage, C., Lieven, E., Theakston, A. \& Tomasello, M. (2003) Testing the abstractness of children's linguistic representations: Lexical and structural priming of syntactic constructions in young children. Developmental Science 6 (5):557-67. doi:10.1111/1467-7687.00312. [aHPB]

Scarborough, D. L., Cortese, C. \& Scarborough, H. S. (1977) Frequency and repetition effects in lexical memory. Journal of Experimental Psychology: Human Perception and Performance 3(1):1-17. [rHPB]

Schacter, D. L. (1987) Implicit memory: History and current status. Journal of Experimental Psychology: Learning, Memory, and Cognition 13(3):501-18. doi:10.1037//0278-7393.13.3.501. [aHPB]

Scheepers, C. (2003) Syntactic priming of relative clause attachments: Persistence of structural configuration in sentence production. Cognition 89:179-205. doi:10.1016/S0010-0277(03)00119-7. [arHPB]

Scheepers, C., Sturt, P., Martin, C. J., Myachykov, A., Teevan, K. \& Viskupova, I. (2011) Structural priming across cognitive domains: From simple arithmetic to relative-clause attachment. Psychological Science 22(10):1319-26. doi:10.1177/ 0956797611416997. [aHPB, NK]

Schiffer, S. (2015) Meaning and formal semantics in generative grammar. Erkenntnis 80(1):61-87. [MG-M]

Schlenker, P., Chemla, E., Arnold, K., Lemasson, A., Ouattara, K., Keenan, S., Stephan, C., Ryder, R. \& Zuberbühler, K. (2014) Monkey semantics: Two "dialects" of Campbell's monkey alarm calls. Linguistics and Philosophy 37 (6):439-501. doi:10.1007/s10988-014-9155-7. [aHPB]

Schmid, H. -J. (2015) A blueprint of the entrenchment-and-conventionalization model. Yearbook of the German Cognitive Linguistics Association 3(1):3-25. Available at: https://doi.org/10.1515/gcla-2015-0002. [FG]

Schmid, M. S. (2011) Language attrition. Cambridge University Press. [GJK]

Schoonbaert, S., Hartsuiker, R. J. \& Pickering, M. J. (2007) The representation of lexical and syntactic information in bilinguals: Evidence from syntactic priming. Journal of Memory and Language 56:153-71. doi:10.1016/j.jml.2006.10.002. [arHPB]

Segaert, K., Kempen, G., Petersson, K. M. \& Hagoort, P. (2013) Syntactic priming and the lexical boost effect during sentence production and sentence comprehension: An fMRI study. Brain and Language 124(2):174-83. doi:10.1016/j. bandl.2012.12.003. [aHPB]

Segaert, K., Menenti, L., Weber, K., Petersson, K. M. \& Hagoort, P. (2012) Shared syntax in language production and language comprehension: An fMRI study. Cerebral Cortex 22(7):1662-70. doi:10.1093/cercor/bhr249. [arHPB, PH]

Segaert, K., Wheeldon, L. \& Hagoort, P. (2016) Unifying structural priming effects on syntactic choices and timing of sentence generation. Journal of Memory and Language 91:59-80. doi:10.1016/j.jml.2016.03.011. [rHPB] 
Seidenberg, M. S. (2007) Connectionist models of reading. In Oxford Handbook of Psycholinguistics, ed. G. Gaskell, pp. 235-50. Oxford University Press. doi:10.1093/oxfordhb/9780198568971.013.0014. [aHPB]

Sevald, C. A., Dell, G. S. \& Cole, J. S. (1995) Syllable structure in speech production: Are syllables chunks or schemas? Journal of Memory and Language 34(6):80720. [aНPB]

Sgall, P., Hajicová, E. \& Panevova, J. (1986) The meaning of the sentence in its semantic and pragmatic aspects. Academia. $[\mathrm{RH}]$

Shibatani, M. (1996) Applicatives and benefactives: A cognitive account. In Grammatical Constructions, ed. M. Shibatani \& S. A. Thompson, pp. 157-96. Clarendon Press. [aHPB]

Shin, J. A. \& Christianson, K. (2009) Syntactic processing in Korean-English bilingual production: Evidence from cross-linguistic structural priming. Cognition 112(1):175-80. doi:10.1016/j.cognition.2009.03.011. [aHPB]

Siew, C. S. \& Vitevitch, M. S. (2016) Spoken word recognition and serial recall of words from components in the phonological network. Journal of Experimental Psychology: Learning, Memory, and Cognition 42(3):394. [ZGC]

Skipper, J. I. (2015) The NOLB model: A model of the natural organization of language and the brain. Cognitive Neuroscience of Natural Language Use 10134. $[\mathrm{AEM}]$

Slevc, L. R. \& Ferreira, V. S. (2013) To err is human, to structurally prime from errors is also human. Journal of Experimental Psychology: Learning, Memory, and Cognition 39(3):985-92. [LRS]

Slevc, L. R. \& Momma, S. (2015) Noisy evidence and plausibility influence structural priming. Poster presented at the Annual Architectures \& Mechanisms for Language Processing (AMLaP) Meeting, Valetta, Malta. [LRS]

Smith, A. C., Monaghan, P. \& Huettig, F. (2017a) Complex word recognition behaviour emerges from the richness of the word learning environment. In: Proceedings of the Fourteenth Neural Computation and Psychology Workshop, vol. 22, ed. K. Twomey, A. C. S. Smith, G. Westermann \& P. Monaghan, pp. 99-114. World Scientific. [CFR]

Smith, A. C., Monaghan, P. \& Huettig, F. (2017b) The multimodal nature of spoken word processing in the visual world: Testing the predictions of alternative models of multimodal integration. Journal of Memory and Language 93:276303. Available at: http://dx.doi.org/10.1016/j.jml.2016.08.005. [CFR]

Smith, M. \& Wheeldon, L. (2001) Syntactic priming in spoken sentence production: An online study. Cognition 78:123-64. doi:10.1016/S0010-0277(00)00110-4. [arHPB, NAL]

Snyder, W. (2000) An experimental investigation of syntactic satiation effects. Linguistic Inquiry 31(3):575-82. doi:10.1162/002438900554479. [arHPB]

Snyder, W. \& Stromswold, K. (1997) The structure and acquisition of English dative constructions. Linguistic Inquiry 28(2):281-317. Available at: https://doi.org/10. 2307/4178978. [CFR]

Sobin, N. (1997) Agreement, default rules, and grammatical viruses. Linguistic Inquiry 28(2):318-43. [aHPB]

Sorace, A. \& Keller, F. (2005) Gradience in linguistic data. Lingua 115:1497-1524. [JZ]

Spector, B. (2007) Scalar implicatures: Exhaustivity and Gricean reasoning. In: Questions in dynamic semantics, current research in the semantics/pragmatics interface, ed. M. Aloni, A. Butler \& P. Dekker, pp. 225-49. Elsevier. [MM]

Spiegel, M. A., Koester, D. \& Schack, T. (2013) The functional role of working memory in the (re-)planning and execution of grasping movements. Journal of Experimental Psychology, Human Perception and Performance 39(5):1326-39. Available at: https://doi.org/10.1037/a0031398. [MCMD]

Spivey, M. \& González-Márquez, M. (2003) Rescuing generative linguistics: Too little, too late? Behavioral and Brain Sciences 26(06):690-1. [MG-M]

Spivey, M., Richardson, D. \& Gonzalez-Marquez, M. (2004) On the perceptualmotor and image-schematic underpinnings of real-time language processing. In: The grounding of cognition: The role of perception and action in memory, language, and thinking, ed. R. Zwaan \& D. Pecher, pp. 246-81. Cambridge University Press. [MG-M]

Sprouse, J. (2008) The differential sensitivity of acceptability judgments to processing effects. Linguistic Inquiry 39(4):686-94. [JS]

Sprouse, J. (2009) Revisiting satiation: Evidence for an equalization response strategy. Linguistic Inquiry 40(2):329-41. doi:10.1162/ling.2009.40.2.329. [rHPB]

Sprouse, J. \& Almeida, D. (2012) Assessing the reliability of textbook data in syntax: Adger's core syntax. Journal of Linguistics 48(03):609-52. [JS, JZ]

Sprouse, J., Caponigro, I., Greco, C. \& Cecchetto, C. (2016) Experimental syntax and the variation of island effects in English and Italian. Natural Language and Linguistic Theory 34:307-44. [LK]

Sprouse, J., Schütze, C. T. \& Almeida, D. (2013) A comparison of informal and formal acceptability judgments using a random sample from linguistic inquiry 2001-2010. Lingua 134: 219-48. doi:10.1016/j.lingua.2013.07.002. [aHPB, JS, JZ]

Sprouse, J., Wagers, M. \& Phillips, C. (2012) A test of the relation between workingmemory capacity and syntactic island effects. Language 88(1):82-123. doi:10.1353/lan.2012.0004. [arHPB, JS]

Steedman, M. (1987) Combinatory grammars and parasitic gaps. Natural Language of Linguistic Theory 5(3):403-39. [aHPB]
Steedman, M. (2000) The syntactic process. MIT Press/Bradford Books. [arHPB, PG] Steedman, M. (2002) Plans, affordances, and combinatory grammar. Linguistics and Philosophy 25(5/6):723-53. doi:10.1023/A:1020820000972. [rHPB]

Stefanowitsch, A. \& Flach, S. (2016) The corpus-based perspective on entrenchment. In: Entrenchment and the psychology of language learning: How we reorganize and adapt linguistic knowledge, ed. H.-J. Schmid, pp. 101-28. de Gruyter Mouton \& the American Psychological Association. Available at: http:// dx.doi.org/10.1037/15969-006. [FG]

Stefanowitsch, A. \& Gries, S. Th. (2003) Collostructions: Investigating the interaction of words and constructions. International Journal of Corpus Linguistics 8:209-43. [NAL]

Steyvers, M. \& Tenenbaum, J. B. (2005) The largescale structure of semantic networks: Statistical analyses and a model of semantic growth. Cognitive Science 29 (1):41-78. [ZGC]

Ströbel, L., ed. (2016) Sensory-motor concepts in language d cognition. Duesseldorf University Press. [MG-M]

Sturt, P. \& Kwon, N. (2015) The processing of raising and nominal control: An eyetracking study. Frontiers in Psychology 6:331. [NK]

Szmrecsanyi, B. (2006) Morphosyntactic persistence in spoken English: A corpus study at the intersection of variationist sociolinguistics, psycholinguistics, and discourse analysis. de Gruyter Mouton. doi:10.1515/9783110197808. [arHPB, NAL]

Tesnière, L. (1959) Éléments de syntaxe structurale. Klincksieck. [RH]

Tesnière, L. (2015) Elements of structural syntax, trans. T., Osborne \& S., Kahane. Benjamins. [RH]

The Five Graces Group (Beckner, C., Blythe, R. A., Bybee, J., Christiansen, M. H. Croft, W., Ellis, N. C., Holland, J., Ke, J., Larsen-Freeman, D. \& Schoenemann, T.) (2009) Language is a complex adaptive system: Position paper. In: Language as a complex adaptive system, ed. N. C. Ellis \& D. Larsen-Freeman, pp. 1-26. Wiley-Blackwell. Available at: http://dx.doi.org/10.1111/j.1467-9922. 2009.00533.x. [FG]

Thompson, S. A. \& Mulac, A. (1991) The discourse conditions for the use of the complementizer "that" in conversational English. Journal of Pragmatics 15:23751. [NAL]

Thothathiri, M. \& Snedeker, J. (2008a) Give and take: Syntactic priming during spoken language comprehension. Cognition 108:51-68. doi:10.1016/j.cognition.2007.12.012. [arHPB, JZ]

Thothathiri, M. \& Snedeker, J. (2008b) Syntactic priming during language comprehension in three- and four-year-old children. Journal of Memory and Language 58(2):188-213. doi:10.1016/j.jml.2007.06.012. [aHPB, CFR, JZ]

Tolman, E. C. (1948) Cognitive maps in rats and men. Psychological Review 55 (4):189-208. [JPdR]

Tomasello, M. (1992) First verbs: A case study of early grammatical development. Cambridge University Press. [aHPB]

Tomasello, M. (1998) The new psychology of language: Cognitive and functional approaches to language structure, vol. 1. Erlbaum. [MG-M]

Tomasello, M. (2000) Do young children have adult syntactic competence? Cognition 74(3):209-53. Available at: https://doi.org/10.1016/S0010-0277(99)000694. [ZGC, CFR]

Tomasello, M. (2003a) Constructing a language: A usage-based theory of language acquisition. [aHPB]

Tomasello, M. (2003b) The new psychology of language: Cognitive and functional approaches to language structure, vol. 2. Erlbaum. [MG-M]

Tomasello, M. (2006) Construction grammar for kids. Constructions 1:1-23. Available at: http://journals.linguisticsociety.org/elanguage/constructions/article/ download/26/26-84-1-PB.pdf. [FG]

Tooley, K. M. \& Bock, K. (2014) On the parity of structural persistence in language production and comprehension. Cognition 132(2):101-36. doi:10.1016/j.cognition.2014.04.002. [aHPB]

Tooley, K. M., Konopka, A. E. \& Watson, D. G. (2014a) Can intonational phrase structure be primed (like syntactic structure)? Journal of Experimental Psychology: Learning, Memory, and Cognition 40(2):348-63. doi:10.1037/ a0034900. [aHPB, JPdR]

Tooley, K. M., Swaab, T. Y., Boudewyn, M. A., Zirnstein, M. \& Traxler, M. J. (2014b) Evidence for priming across intervening sentences during on-line sentence comprehension. Language and Cognitive Processes 29(3):289-311. Available at: http://doi.org/10.1080/01690965.2013.770892. [RR]

Tooley, K. M. \& Traxler, M. J. (2010). Syntactic priming effects in comprehension: A critical review. Language and Linguistics Compass 4(10):925-37. Available at: http://doi.org/10.1111/j.1749-818X.2010.00249.x. [RR]

Traxler, M. J. (2014) Trends in syntactic parsing: Anticipation, Bayesian estimation, and good-enough parsing. Trends in Cognitive Sciences 18(11):605-11. [LRS]

Traxler, M. J. \& Pickering, M. (1996) Plausibility and the processing of unbounded dependencies: An eye-tracking study. Journal of Memory and Language 35 (3):454-75. [aHPB]

Traxler, M. J., Tooley, K. M. \& Pickering, M. J. (2014) Syntactic priming during sentence comprehension: Evidence for the lexical boost. Journal of Experimental Psychology. Learning, Memory, and Cognition 40(4):905-18. doi:10.1037/a0036377. [arHPB] 
References/Branigan and Pickering: An experimental approach to linguistic representation

Vallduvi, E. (1992) The Information Component. Garland Press. [aHPB]

van Beijsterveldt, L. M. \& van Hell, J. G. (2009) Structural priming of adjective-noun structures in hearing and deaf children. Journal of Experimental Child Psychology 104(2):179-96. doi:10.1016/j.jecp.2009.05.002. [aHPB]

Van der Cavey, J. \& Hartsuiker, R. J. (2016) Is there a domain-general cognitive structuring system? Evidence from structural priming across music, math, action descriptions, and language. Cognition 146:172-84. doi: https://doi.org/10. 1016/j.cognition.2015.09.013. [arHPB, MCMD]

Van Gompel, R. P. G., Pickering, M. J., Pearson, J. \& Jacob, G. (2006) The activation of inappropriate analyses in garden-path sentences: Evidence from structural priming. Journal of Memory and Language 55(3):335-62. doi:10.1016/j. jml.2006.06.004. [LRS, rHPB]

Van Patten, B. (2014) The psycholinguistics of SLA. In: Research methods in second language psycholinguistics, ed. J. Jegerski \& B. VanPatten, pp. 1-19. Routledge. [ZGC]

Van Petten, C., Coulson, S., Plante, E., Rubin, S. \& Parks, M. (1999) Timecourse of word identification and semantic integration in spoken language. Journal of Experimental Psychology: Learning, Memory, and Cognition 25(2):394-417. [MG-M]

van Rooij, R. \& Schulz, K. (2004) Exhaustive interpretation of complex sentences. Journal of Logic, Language and Information 13:491-519. [MM]

Vasilyeva, M. \& Waterfall, H. (2012) Beyond syntactic priming: Evidence for activation of alternative syntactic structures. Journal of Child Language 39(2):25883. doi:10.1017/S0305000911000055. [aHPB]

Vasilyeva, M., Waterfall, H., Gámez, P. B., Gómez, L. E., Bowers, E. \& Shimpi, P. (2010) Cross-linguistic syntactic priming in bilingual children. Journal of Child Language 37(5):1047-64. doi:10.1017/S0305000909990213. [aHPB]

Vernice, M., Pickering, M. J. \& Hartsuiker, R. J. (2012) Thematic emphasis in language production. Language and Cognitive Processes 27(5):631-64. doi:10.1080/01690965.2011.572468. [aHPB, RJH]

Viau, J., Lidz, J. \& Musolino, J. (2010) Priming of abstract logical representations in 4-year-olds. Language Acquisition 17:26-50. doi:10.1080/10489221003620946. [aHPB, WOG]

Walker, E., Bergen, B. \& Núñez, R. (2013) Investigating spatial axis recruitment in temporal reckoning through acoustic stimuli and non-spatial responses. Center for Research in Language Technical Report, University of California, San Diego 25:1-10. [MG-M]

Wasow, T. \& Arnold, J. (2005) Intuitions in linguistic argumentation. Lingua 115 (11):1481-96. doi:10.1016/j.lingua.2004.07.001. [aHPB]
Wasow, T., Jaeger, T. F. \& Orr, D. M. (2011) Lexical variation in relativizer frequency. In: Expecting the unexpected: Exceptions in grammar, ed. H. J. Simon $\&$ H. Wiese, pp. 175-96. deGruyter. [NAL]

Weiss, D. J. \& Wark, J. (2009) Hysteresis effect in a motor task in cotton-top tamarins (Saguinusoedipus). Journal of Experimental Psychology: Animal Behavior Processes 35(3):135-41. [MCMD]

Wells, J. B., Christiansen, M. H., Race, D. S. \& MacDonald, M. C. (2009) Experience and sentence processing: Statistical learning and relative clause comprehension. Cognitive Psychology 58(2):250-71. doi: http://doi.org/10.1016/j. cogpsych.2008.08.002. [RR]

Wilks, C. \& Meara, P. (2002) Untangling word webs: Graph theory and the notion of density in second language word association networks. Second Language Research 18(4):303-24. [ZGC]

Winawer, J., Huk, A. \& Boroditsky, L. (2008) A motion aftereffect from still photographs depicting motion. Psychological Science 19(3):276-83. [MG-M]

Winter, B. \& Matlock, T. (2013) Making judgments based on similarity and proximity. Metaphor \& Symbol 28:1-14. [MG-M]

Wittenberg, E. (2014) With light verb constructions from syntax to concepts. Unpublished doctoral dissertation, University of Potsdam. [aHPB, EJF, KM]

Wittenberg, E., Jackendoff, R., Kuperberg, G., Paczynski, M., Snedeker, J. \& Wiese, H. (2014) The processing and representation of light verb constructions. In: Structuring the argument, ed. A. Bachrach, I. Roy \& L. Stockall, pp. 61-80. John Benjamins. [aHPB]

Yoon, J. \& Gries, S. Th., eds. (2016) Corpus-based approaches to construction grammar. John Benjamins. [MG-M]

Yoshida, M., Kazanina, N., Pablos, L. \& Sturt, P. (2014) On the origin of islands. Language, Cognition and Neuroscience 29(7):761-70. [JS]

Zaenen, A. E. (1988) Unaccusative verbs in Dutch and the syntax-semantics interface. CSLI/SRI International 123:317-35. [rHPB]

Zervakis, J. \& Mazuka, R. (2013) Effect of repeated evaluation and repeated exposure on acceptability ratings of sentences. Journal of Psycholinguistic Research 42(6):505-25. doi:10.1007/s10936-012-9233-3. [aHPB]

Ziegler, J. \& Snedeker, J. (2016a) Structural priming across development: The lexical boost, abstract priming, and task demands. Poster presented at the Forty-first Boston University Conference on Language Development, Boston, MA. [JZ]

Ziegler, J. \& Snedeker, J. (2016b) Toward a comprehensive view of structural priming: What gets primed when. Poster presented at the Twenty-ninth Annual Meeting of the CUNY Conference on Human Sentence Processing, Gainesville, FL. [JZ] 